\title{
Visible-Light Driven Selective C-N Bond Scission in anti-Bimane-Like Derivatives
}

Nejc Petek, Helena Brodnik, Uroš Grošelj, Jurij Svete, Franc Požgan, Bogdan Štefane*

Faculty of Chemistry and Chemical Technology, University of Ljubljana, Večna pot 113, 1000 Ljubljana, Slovenia

\section{Table of contents}

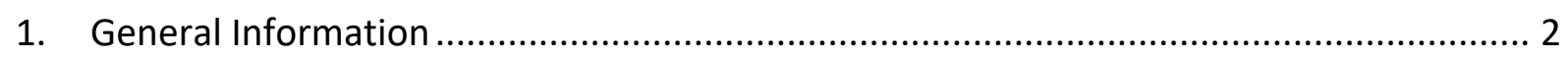

2. Preparation and characterization of azomethine imines (AMI) and compounds 1 ........... 3

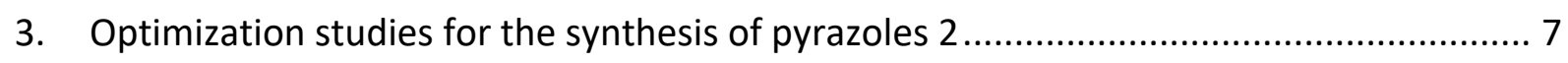

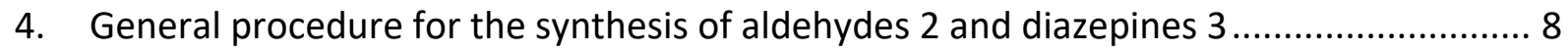

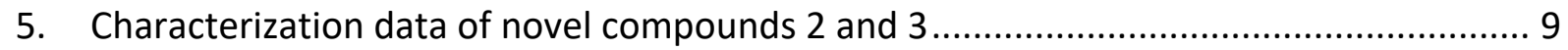

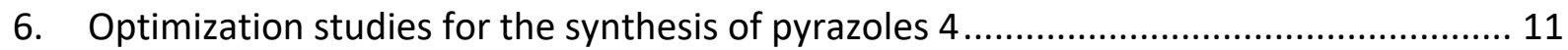

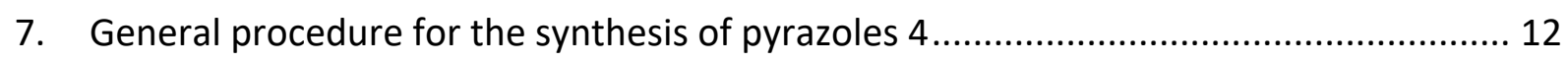

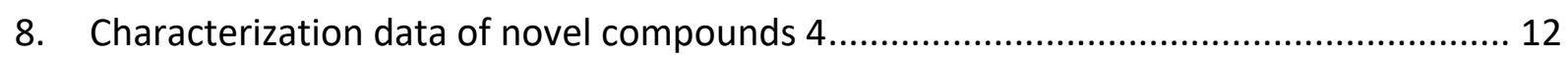

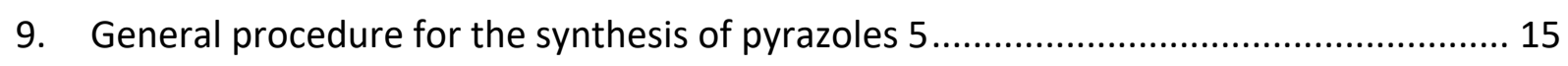

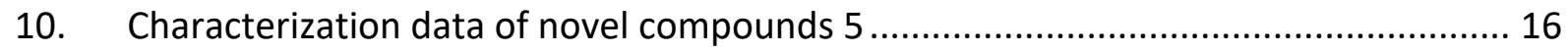

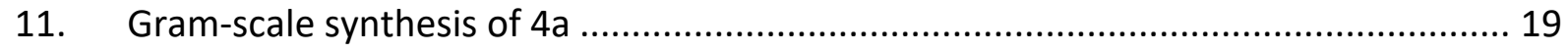

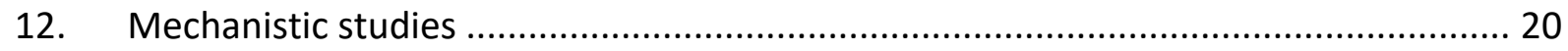

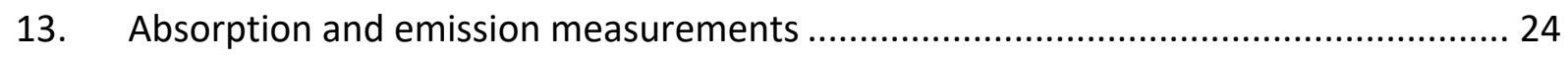

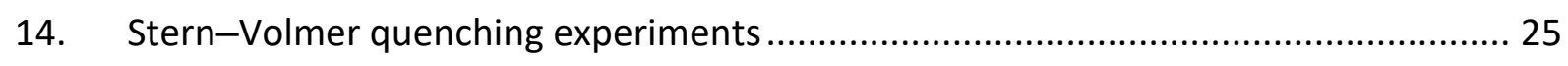

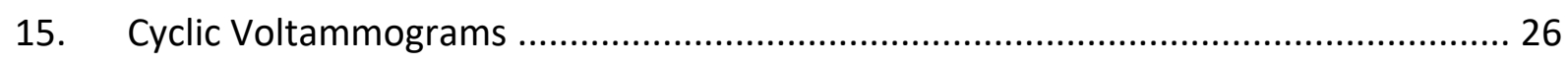

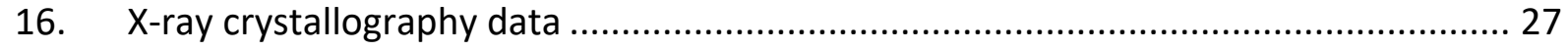

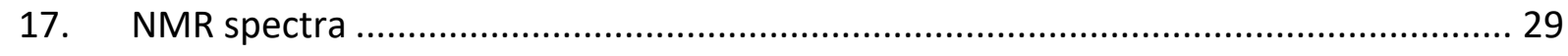

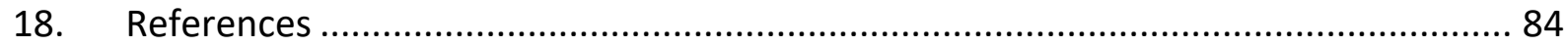




\section{General Information}

Reactions were carried out in borosilicate vials in a commercially available SynLED Parallel Photoreactor (465-470 nm, 130-140 Im, Sigma-Aldrich, St. Louis, MO, USA), and a custom made photoreactor, cooled with a cooling block to sustain a reaction temperature of $25{ }^{\circ} \mathrm{C}$ (room temperature). Vials are placed approximately $2 \mathrm{~mm}$ above LEDs with no filter applied. LEDs used: $400 \mathrm{~nm}$ (Edison, LT-1467, UV, wavelength of peak intensity 395-410 nm, radiantflux $350 \mathrm{~mW}$ ), $450 \mathrm{~nm}$ (ProLight Opto, PM2B-3-LBS-SD, blue, wavelength of peak intensity 445-455 nm, 39.8-51.7 Im) and $510 \mathrm{~nm}$ (Seoul Semiconductor, G42180, green, wavelength of peak intensity $505-520 \mathrm{~nm}, 70 \mathrm{Im}$ ). Cyclic voltammograms were recorded on ElectraSyn 2.0 (IKA ${ }^{\circledR}$-Werke $\mathrm{GmbH} \&$ Co. KG, Staufen, Germany) on glassy carbon working electrode with Pt plated counter electrode and Ag wire quasi-reference electrode. Anhydrous solvents were used. The NMR spectra were recorded in deuterated solvents with $\mathrm{Me}_{4} \mathrm{Si}$ as the internal standard on a Bruker Avance III UltraShield 500 plus instruments (Bruker, Billerica, MA, USA) at $500 \mathrm{MHz}$ for ${ }^{1} \mathrm{H}$ and at $126 \mathrm{MHz}$ for ${ }^{13} \mathrm{C}$ nuclei, respectively. Data for ${ }^{1} \mathrm{H}$ NMR are reported as chemical shifts $(\delta)$ in ppm, multiplicity (bs = broad singlet, $s=$ singlet, $d=$ doublet, $\mathrm{t}=$ triplet, $\mathrm{q}=$ quartet, $\mathrm{m}=$ multiplet), coupling constant and integration. Data for ${ }^{13} \mathrm{C}$ are reported as chemical shift $(\delta)$ in ppm. Mass spectra were recorded on Agilent 6224 Accurate Mass TOF LC/MS spectrometer (Agilent Technologies, Santa Clara, CA, USA) and IR spectra on a Bruker FTIR Alpha Platinum spectrophotometer (Bruker, Billerica, MA, USA). Melting points were determined on a Kofler hot-stage microscope. Absorption spectra were recorded on Cary 50 Bio UV-VIS Spectrophotometer (Agilent Technologies, Santa Clara, CA, USA). Photoluminescence spectra were recorded on Cary Eclipse Fluorescence Spectrophotometer (Agilent Technologies, Santa Clara, CA, USA). Thin-layer chromatography (TLC) was performed on aluminum backed silica plates $(0.2 \mathrm{~mm}, 60 \mathrm{~F} 254$, Sigma-Aldrich, St. Louis, MO, USA). Visualization of TLC (254 nm, Camag, Muttenz, Switzerland) was performed by fluorescence quenching or with potassium permanganate stains. Column chromatography (CC) was performed on silica gel (particle size: 35-70 $\mu \mathrm{m}$, Sigma-Aldrich, St. Louis, MO, USA). Commercially available compounds were used without further purification. Compounds AMI and $\mathbf{1}$ were prepared according to the established literature procedures as described herein. 


\section{Preparation and characterization of azomethine imines (AMI) and compounds 1}

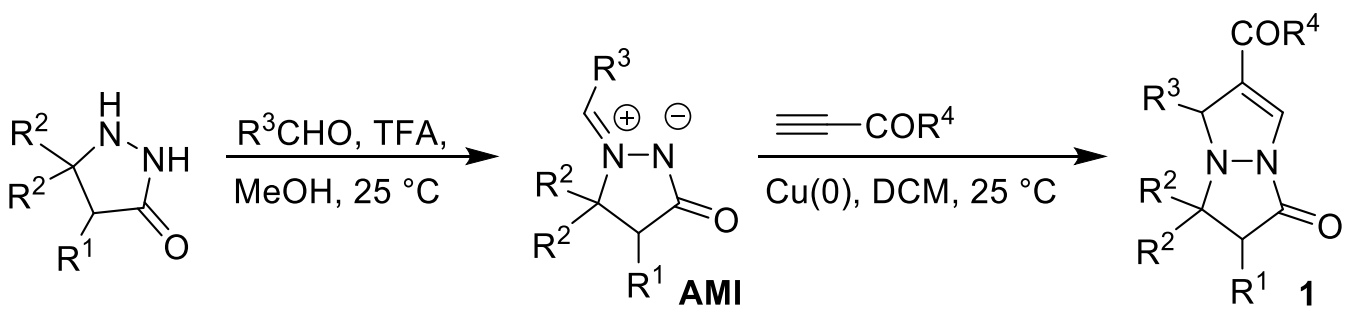

Scheme SI1: Reaction scheme for the preparation of azomethine imines and pyrazolo[1,2a]pyrazoles 1.

Compounds AMI1-15 were prepared according to established literature procedures. ${ }^{1} \mathrm{H}$ NMR data of known compounds are in agreement with reported values.

Compounds 1a-r were prepared according to the procedure, which has been slightly modified from the literature. ${ }^{9} \mathrm{~A}$ mixture of AMI $(2.0 \mathrm{mmol})$, dipolarophile $(2.4 \mathrm{mmol})$, copper powder (80 mg), and $\mathrm{CH}_{2} \mathrm{Cl}_{2}(10 \mathrm{~mL})$ was stirred at $25^{\circ} \mathrm{C}$ for $24 \mathrm{~h}$. The catalyst and impurities were removed by $\mathrm{CC}(\mathrm{DCM} / \mathrm{MeOH}, 40: 1)$ to give pure products 1 .

(Z)-1-(4-Chlorobenzylidene)-5,5-dimethyl-3-oxopyrazolidin-1-ium-2-ide (AMI1). ${ }^{1}$

(Z)-1-Benzylidene-5,5-dimethyl-3-oxopyrazolidin-1-ium-2-ide (AMI2). ${ }^{2}$

(Z)-1-(4-Methoxybenzylidene)-5,5-dimethyl-3-oxopyrazolidin-1-ium-2-ide (AMI3). ${ }^{1}$

(Z)-1-(4-Cyanobenzylidene)-5,5-dimethyl-3-oxopyrazolidin-1-ium-2-ide (AMI4). ${ }^{1}$

(Z)-1-(Furan-2-ylmethylene)-5,5-dimethyl-3-oxopyrazolidin-1-ium-2-ide (AMI5). ${ }^{3}$

(Z)-1-Ethylidene-5,5-dimethyl-3-oxopyrazolidin-1-ium-2-ide (AMI6). ${ }^{4}$

(Z)-1-Benzylidene-3-oxopyrazolidin-1-ium-2-ide (AMI7). ${ }^{5}$

(Z)-3,3-Dimethyl-5-oxo-2-[(E)-3-phenylallylidene]pyrazolidin-2-ium-1-ide (AMI8). ${ }^{6}$

(Z)-5,5-Dimethyl-1-(4-methylbenzylidene)-3-oxopyrazolidin-1-ium-2-ide (AMI9). ${ }^{1}$

(Z)-1-(2,6-Dichlorobenzylidene)-5,5-dimethyl-3-oxopyrazolidin-1-ium-2-ide (AMI10). ${ }^{7}$

(Z)-5,5-Dimethyl-3-oxo-1-(3,4,5-trimethoxybenzylidene)pyrazolidin-1-ium-2-ide (AMI11). ${ }^{7}$

(Z)-5,5-Dimethyl-1-(naphthalen-2-ylmethylene)-3-oxopyrazolidin-1-ium-2-ide (AMI12). ${ }^{6}$

(Z)-1-Benzylidene-4-(benzyloxycarbonylamino)-5,5-dimethyl-3-oxopyrazolidin-1-ium-2-ide (AMI13). ${ }^{8}$

(Z)-5,5-dimethyl-3-oxo-1-((1-tosyl-1H-indol-3-yl)methylene)pyrazolidin-1-ium-2-ide (AMI14). Prepared according to an established literature procedure ${ }^{7}$ from 5,5-dimethylpyrazolidin-3-one $(570 \mathrm{mg}, 5.0 \mathrm{mmol}$ ) and 1-tosyl-1H-indole-3-carbaldehyde $(1.57 \mathrm{~g}, 5.25 \mathrm{mmol}) ; 1.364 \mathrm{~g}$ (69\% yield); light brown solid, $\mathrm{mp} 163-164{ }^{\circ} \mathrm{C} ; v_{\max } / \mathrm{cm}^{-1}$ (ATR) 
1652, 1589, 1172, 1091, 667; $\delta_{\mathrm{H}}\left(500 \mathrm{MHz} ; \mathrm{CDCl}_{3} ; \mathrm{Me}_{4} \mathrm{Si}\right) 9.22(\mathrm{~s}, 1 \mathrm{H}), 8.05(\mathrm{~d}, J=8.3 \mathrm{~Hz}, 1 \mathrm{H})$, $7.87(\mathrm{~d}, J=8.3 \mathrm{~Hz}, 2 \mathrm{H}), 7.68(\mathrm{~d}, J=7.8 \mathrm{~Hz}, 1 \mathrm{H}), 7.41(\mathrm{t}, J=7.6 \mathrm{~Hz}, 1 \mathrm{H}), 7.35(\mathrm{t}, J=7.5 \mathrm{~Hz}, 1 \mathrm{H})$, $7.30(\mathrm{~s}, 1 \mathrm{H}), 7.25(\mathrm{~d}, J=8.3 \mathrm{~Hz}, 2 \mathrm{H}), 2.81(\mathrm{~s}, 2 \mathrm{H}), 2.34(\mathrm{~s}, 3 \mathrm{H}), 1.75(\mathrm{~s}, 6 \mathrm{H}) ; \delta_{\mathrm{c}}\left(126 \mathrm{MHz} ; \mathrm{CDCl}_{3}\right.$; $\left.\mathrm{Me}_{4} \mathrm{Si}\right)$ 181.5, 145.7, 134.7, 134.3, 132.6, 130.1, 128.0, 127.2, 125.8, 124.0, 120.2, 117.9, 114.0, 111.7, 72.5, 45.2, 29.0, 21.6; HRMS (ESI) m/z: [M+ H] $]^{+}$Calcd for $\mathrm{C}_{21} \mathrm{H}_{22} \mathrm{~N}_{3} \mathrm{O}_{3} \mathrm{~S}$ 396.1376; Found 396.1369 .

(Z)-5,5-dimethyl-1-((1-methyl-1H-pyrrol-2-yl)methylene)-3-oxopyrazolidin-1-ium-2-ide

(AMI15). Prepared according to an established literature procedure ${ }^{7}$ from 5,5-dimethylpyrazolidin-3-one $(570 \mathrm{mg}, 5.0 \mathrm{mmol}$ ) and 1-methylpyrrole-2-carboxaldehyde (573 mg, $5.25 \mathrm{mmol}$ ); additionally purified by CC (DCM/MeOH, 10:1); $749 \mathrm{mg}$ (73\% yield); light brown solid, $\mathrm{mp} 188-189^{\circ} \mathrm{C} ; v_{\max } / \mathrm{cm}^{-1}$ (ATR) 3081, 2970, 1644, 1602, 1324, 1308, 1072, 1052, $682 ; \delta_{\mathrm{H}}\left(500 \mathrm{MHz} ; \mathrm{CDCl}_{3} ; \mathrm{Me}_{4} \mathrm{Si}\right) 7.87(\mathrm{dd}, J=4.1,1.5 \mathrm{~Hz}, 1 \mathrm{H}), 7.14(\mathrm{~s}, 1 \mathrm{H}), 6.92-6.88(\mathrm{~m}, 1 \mathrm{H})$, $6.29(\mathrm{dd}, J=4.0,2.6 \mathrm{~Hz}, 1 \mathrm{H}), 3.82(\mathrm{~s}, 3 \mathrm{H}), 2.69(\mathrm{~s}, 2 \mathrm{H}), 1.68(\mathrm{~s}, 6 \mathrm{H}) ; \delta_{c}\left(126 \mathrm{MHz} ; \mathrm{CDCl}_{3} ; \mathrm{Me}_{4} \mathrm{Si}\right)$ $180.8,129.8,124.5,121.88,121.85,119.4,110.5,71.4,45.4,34.5,28.8 ;$ HRMS (ESI) m/z: $[\mathrm{M}+\mathrm{H}]^{+}$Calcd for $\mathrm{C}_{11} \mathrm{H}_{16} \mathrm{~N}_{3} \mathrm{O}$ 206.1288; Found 206.1289.

Methyl 1-(4-chlorophenyl)-7,7-dimethyl-5-oxo-6,7-dihydro-1H,5H-pyrazolo[1,2-a]pyrazole-2carboxylate (1a). Prepared according to the general procedure from AMI1. ${ }^{1} \mathrm{H}$ NMR data is in agreement with the reported values. ${ }^{9}$

Methyl 7,7-dimethyl-5-oxo-1-phenyl-6,7-dihydro-1H,5H-pyrazolo[1,2-a]pyrazole-2carboxylate (1b). Prepared according to the general procedure from AMI2. ${ }^{1} \mathrm{H}$ NMR data is in agreement with the reported values. ${ }^{9}$

Methyl 1-(4-methoxyphenyl)-7,7-dimethyl-5-oxo-6,7-dihydro-1H,5H-pyrazolo[1,2-a]pyrazole2-carboxylate (1c). Prepared according to the general procedure from AMI3 (464 mg, 2.0 $\mathrm{mmol}$ ) and methyl propiolate $(214 \mu \mathrm{L}, 2.4 \mathrm{mmol}) ; 485 \mathrm{mg}$ (77\% yield); yellow solid, $\mathrm{mp} 86-87$ ${ }^{\circ} \mathrm{C} ; v_{\max } / \mathrm{cm}^{-1}$ (ATR) 3080, 1689, 1601, 1325, 1262, 1247, 1224, 1192, 1170; $\delta_{\mathrm{H}}\left(500 \mathrm{MHz} ; \mathrm{CDCl}_{3}\right.$; $\left.\mathrm{Me}_{4} \mathrm{Si}\right) 7.49(\mathrm{~d}, J=1.5 \mathrm{~Hz}, 1 \mathrm{H}), 7.38-7.34(\mathrm{~m}, 2 \mathrm{H}), 6.90-6.85(\mathrm{~m}, 2 \mathrm{H}), 5.43(\mathrm{~d}, J=1.2 \mathrm{~Hz}, 1 \mathrm{H})$, $3.80(\mathrm{~s}, 3 \mathrm{H}), 3.62(\mathrm{~s}, 3 \mathrm{H}), 2.86(\mathrm{~d}, J=15.7 \mathrm{~Hz}, 1 \mathrm{H}), 2.39(\mathrm{~d}, J=15.7 \mathrm{~Hz}, 1 \mathrm{H}), 1.23(\mathrm{~s}, 3 \mathrm{H}), 1.15(\mathrm{~s}$, $3 \mathrm{H}) ; \delta_{\mathrm{C}}\left(126 \mathrm{MHz} ; \mathrm{CDCl}_{3} ; \mathrm{Me}_{4} \mathrm{Si}\right)$ 166.4, 164.2, 159.2, 134.1, 129.2, 128.9, 117.0, 113.8, 64.3, 64.0, 55.2, 51.5, 49.5, 25.0, 19.0; HRMS (ESI) m/z: $[\mathrm{M}+\mathrm{H}]^{+}$Calcd for $\mathrm{C}_{17} \mathrm{H}_{21} \mathrm{~N}_{2} \mathrm{O}_{4}$ 317.1496; Found 317.1492.

Methyl 1-(4-cyanophenyl)-7,7-dimethyl-5-oxo-6,7-dihydro-1H,5H-pyrazolo[1,2-a]pyrazole-2carboxylate (1d). Prepared according to the general procedure from AMI4 (454 mg, $2.0 \mathrm{mmol}$ ) and methyl propiolate ( $214 \mu \mathrm{L}, 2.4 \mathrm{mmol}) ; 380 \mathrm{mg}$ (61\% yield); yellow solid, $\mathrm{mp} 104-105^{\circ} \mathrm{C}$; $v_{\max } / \mathrm{cm}^{-1}$ (ATR) 2229, 1740, 1684, 1600, 1327, 1197; $\delta_{\mathrm{H}}\left(500 \mathrm{MHz} ; \mathrm{CDCl}_{3} ; \mathrm{Me}_{4} \mathrm{Si}\right) 7.69-7.59$ $(\mathrm{m}, 4 \mathrm{H}), 7.52(\mathrm{~d}, J=1.4 \mathrm{~Hz}, 1 \mathrm{H}), 5.52(\mathrm{~d}, J=1.1 \mathrm{~Hz}, 1 \mathrm{H}), 3.64(\mathrm{~s}, 3 \mathrm{H}), 2.88(\mathrm{~d}, J=15.8 \mathrm{~Hz}, 1 \mathrm{H})$, $2.43(\mathrm{~d}, J=15.8 \mathrm{~Hz}, 1 \mathrm{H}), 1.24(\mathrm{~s}, J=18.0 \mathrm{~Hz}, 3 \mathrm{H}), 1.13(\mathrm{~s}, J=20.0 \mathrm{~Hz}, 3 \mathrm{H}) ; \delta_{\mathrm{C}}\left(126 \mathrm{MHz} ; \mathrm{CDCl}_{3}\right.$; $\left.\mathrm{Me}_{4} \mathrm{Si}\right)$ 166.5, 163.8, 147.2, 132.3, 129.9, 128.7, 118.8, 115.7, 111.8, 64.6, 64.1, 51.7, 49.1, 24.9, 19.0; HRMS (ESI) m/z: [M + H] $]^{+}$Calcd for $\mathrm{C}_{17} \mathrm{H}_{18} \mathrm{~N}_{3} \mathrm{O}_{3}$ 312.1343; Found 312.1337.

Methyl 1-(furan-2-yl)-7,7-dimethyl-5-oxo-6,7-dihydro-1H,5H-pyrazolo[1,2-a]pyrazole-2carboxylate (1e). Prepared according to the general procedure from AMI5 (384 mg, 2.0 
$\mathrm{mmol}$ ) and methyl propiolate ( $214 \mu \mathrm{L}, 2.4 \mathrm{mmol}) ; 418 \mathrm{mg}$ (76\% yield); yellow solid, $\mathrm{mp} 131-$ $132{ }^{\circ} \mathrm{C} ; v_{\max } / \mathrm{cm}^{-1}$ (ATR) 3078, 1732, 1688, 1601, 1206, 748; $\delta_{\mathrm{H}}\left(500 \mathrm{MHz} ; \mathrm{CDCl}_{3} ; \mathrm{Me}_{4} \mathrm{Si}\right) 7.57$ $(\mathrm{d}, J=1.5 \mathrm{~Hz}, 1 \mathrm{H}), 7.40(\mathrm{~d}, J=1.1 \mathrm{~Hz}, 1 \mathrm{H}), 6.35(\mathrm{dd}, J=3.2,1.9 \mathrm{~Hz}, 1 \mathrm{H}), 6.27(\mathrm{~d}, J=3.2 \mathrm{~Hz}$, $1 \mathrm{H}), 5.58(\mathrm{~d}, J=1.2 \mathrm{~Hz}, 1 \mathrm{H}), 3.70(\mathrm{~s}, 3 \mathrm{H}), 2.87(\mathrm{~d}, J=15.7 \mathrm{~Hz}, 1 \mathrm{H}), 2.39(\mathrm{~d}, J=15.7 \mathrm{~Hz}, 1 \mathrm{H})$, $1.31(\mathrm{~s}, 3 \mathrm{H}), 1.22(\mathrm{~s}, 3 \mathrm{H}) ; \delta_{c}\left(126 \mathrm{MHz} ; \mathrm{CDCl}_{3} ; \mathrm{Me}_{4} \mathrm{Si}\right)$ 166.8, 164.0, 153.5, 142.6, 131.1, 113.2, 110.6, 107.3, 64.6, 58.1, 51.7, 49.1, 24.7, 18.8; HRMS (ESI) m/z: [M + H] Calcd for $\mathrm{C}_{14} \mathrm{H}_{17} \mathrm{~N}_{2} \mathrm{O}_{4}$ 277.1183; Found 277.1182.

Methyl 1,7,7-trimethyl-5-oxo-6,7-dihydro-1H,5H-pyrazolo[1,2-a]pyrazole-2-carboxylate (1f). Prepared according to the general procedure from AMI6. ${ }^{1} \mathrm{H}$ NMR data is in agreement with the reported values. ${ }^{3}$

Methyl 5-oxo-1-phenyl-6,7-dihydro-1H,5H-pyrazolo[1,2-a]pyrazole-2-carboxylate (1g). Prepared according to the general procedure from AMI7. ${ }^{1} \mathrm{H}$ NMR data is in agreement with the reported values. ${ }^{10}$

Methyl (E)-7,7-dimethyl-5-oxo-1-(prop-1-en-1-yl)-6,7-dihydro-1H,5H-pyrazolo[1,2-a]pyrazole -2-carboxylate (1h). Prepared by stirring a solution of 5,5-dimethylpyrazolidin-3-one $(570 \mathrm{mg}$, $5.0 \mathrm{mmol})$, crotonaldehyde $(435 \mu \mathrm{L}, 5.25 \mathrm{mmol})$ and TFA $(10 \mu \mathrm{L})$ in $\mathrm{MeOH}(10 \mathrm{~mL})$ at $25^{\circ} \mathrm{C}$ for $6 \mathrm{~h}$. After evaporating the volatiles, copper powder $(200 \mathrm{mg}), \mathrm{CH}_{2} \mathrm{Cl}_{2}(25 \mathrm{~mL})$ and methyl propiolate $(535 \mu \mathrm{L}, 6.0 \mathrm{mmol})$ were added and the mixture was stirred at $25^{\circ} \mathrm{C}$ for $24 \mathrm{~h}$. The catalyst and impurities were removed by $\mathrm{CC}$ on silica (DCM/MeOH, 40:1) and the filtrate evaporated in vacuo to give $1 \mathrm{~h}$ as an oil, which slowly crystalized. $850 \mathrm{mg}$ ( $68 \%$ yield); yellow solid; $\mathrm{mp} 57-58^{\circ} \mathrm{C} ; v_{\max } / \mathrm{cm}^{-1}$ (ATR) 2966, 1731, 1695, 1598; $\delta_{\mathrm{H}}\left(500 \mathrm{MHz} ; \mathrm{CDCl}_{3} ; \mathrm{Me}_{4} \mathrm{Si}\right) 7.39$ (d, $J=1.5 \mathrm{~Hz}, 1 \mathrm{H}$ ), 5.78 (dqd, $J=13.0,6.5,0.7 \mathrm{~Hz}, 1 \mathrm{H}), 5.55$ (ddq $J=15.1,6.8,1.5 \mathrm{~Hz}, 1 \mathrm{H}$ ), 4.93 (d, $J=6.9 \mathrm{~Hz}, 1 \mathrm{H}), 3.73(\mathrm{~s}, 3 \mathrm{H}), 2.84(\mathrm{~d}, J=15.7 \mathrm{~Hz}, 1 \mathrm{H}), 2.36(\mathrm{~d}, J=15.6 \mathrm{~Hz}, 1 \mathrm{H}), 1.73(\mathrm{dd}, J=$ 6.4, $1.3 \mathrm{~Hz}, 3 \mathrm{H}), 1.31$ (s, 3H), 1.14 (s, 3H); $\delta_{\mathrm{c}}\left(126 \mathrm{MHz} ; \mathrm{CDCl}_{3} ; \mathrm{Me}_{4} \mathrm{Si}\right)$ 166.6, 164.3, 130.7, 129.7, 128.2, 115.9, 64.4, 62.5, 51.5, 49.4, 25.0, 19.0, 17.7; HRMS (ESI) m/z: [M + H] Calcd for $\mathrm{C}_{13} \mathrm{H}_{19} \mathrm{~N}_{2} \mathrm{O}_{3}$ 251.1390; Found 251.1389.

Methyl (E)-7,7-dimethyl-5-oxo-1-styryl-6,7-dihydro-1H,5H-pyrazolo[1,2-a]pyrazole-2carboxylate (1i). Prepared according to the general procedure from AMI8 (456 mg, $2.0 \mathrm{mmol}$ ) and methyl propiolate $(214 \mu \mathrm{L}, 2.4 \mathrm{mmol}) ; 620 \mathrm{mg}$ (99\% yield); yellow oil; $v_{\max } / \mathrm{cm}^{-1}$ (ATR) 2975, 1696, 1600, 1205, 728; $\delta_{\mathrm{H}}\left(500 \mathrm{MHz} ; \mathrm{CDCl}_{3} ; \mathrm{Me}_{4} \mathrm{Si}\right) 7.44$ (d, J = $\left.1.5 \mathrm{~Hz}, 1 \mathrm{H}\right), 7.43-7.39$ (m, 2H), $7.34-7.29(\mathrm{~m}, 2 \mathrm{H}), 7.26-7.22(\mathrm{~m}, 1 \mathrm{H}), 6.73(\mathrm{~d}, J=15.7 \mathrm{~Hz}, 1 \mathrm{H}), 6.32(\mathrm{dd}, J=15.8$, $6.6 \mathrm{~Hz}, 1 \mathrm{H}), 5.15(\mathrm{~d}, J=6.6 \mathrm{~Hz}, 1 \mathrm{H}), 3.72(\mathrm{~s}, 3 \mathrm{H}), 2.89(\mathrm{~d}, J=15.7 \mathrm{~Hz}, 1 \mathrm{H}), 2.40(\mathrm{~d}, J=15.7 \mathrm{~Hz}$, 1H), 1.33 (s, 3H), 1.18 (s, 3H); $\delta_{C}\left(126 \mathrm{MHz} \mathrm{CDCl}_{3}\right.$; Me $\left.{ }_{4} \mathrm{Si}\right)$ 166.7, 164.2, 136.6, 131.7, 129.8, 128.7, 128.6, 127.8, 126.7, 115.7, 64.5, 62.6, 51.6, 49.4, 25.0, 19.1; HRMS (ESI) m/z: $\left[\mathrm{M}+\mathrm{H}^{+}\right.$ Calcd for $\mathrm{C}_{18} \mathrm{H}_{21} \mathrm{~N}_{2} \mathrm{O}_{3}$ 313.1547; Found 313.1544.

Methyl 7,7-dimethyl-1-(4-methylphenyl)-5-oxo-6,7-dihydro-1H,5H-pyrazolo[1,2-a]pyrazole2-carboxylate (1j). Prepared according to the general procedure from AMI9 (432 mg, 2.0 $\mathrm{mmol}$ ) and methyl propiolate $(214 \mu \mathrm{L}, 2.4 \mathrm{mmol}) ; 440 \mathrm{mg}$ (73\% yield); yellow solid, $\mathrm{mp} 154-$ $155^{\circ} \mathrm{C} ; v_{\text {max }} / \mathrm{cm}^{-1}$ (ATR) 3081, 1685, 1601, 1191; $\delta_{\mathrm{H}}\left(500 \mathrm{MHz} ; \mathrm{CDCl}_{3} ; \mathrm{Me}_{4} \mathrm{Si}\right) 7.50$ (d, $J=1.4 \mathrm{~Hz}$, $1 \mathrm{H}), 7.32(\mathrm{~d}, J=8.0 \mathrm{~Hz}, 2 \mathrm{H}), 7.15(\mathrm{~d}, J=7.9 \mathrm{~Hz}, 2 \mathrm{H}), 5.43(\mathrm{~s}, 1 \mathrm{H}), 3.62(\mathrm{~s}, 3 \mathrm{H}), 2.86(\mathrm{~d}, J=15.7$ $\mathrm{Hz}, 1 \mathrm{H}), 2.38(\mathrm{~d}, J=15.7 \mathrm{~Hz}, 1 \mathrm{H}), 2.34(\mathrm{~s}, 3 \mathrm{H}), 1.23(\mathrm{~s}, 3 \mathrm{H}), 1.15(\mathrm{~s}, 3 \mathrm{H}) ; \delta_{\mathrm{c}}\left(126 \mathrm{MHz} ; \mathrm{CDCl}_{3}\right.$; 
$\left.\mathrm{Me}_{4} \mathrm{Si}\right)$ 166.6, 164.2, 139.1, 137.6, 129.4, 129.2, 127.7, 116.9, 64.5, 64.3, 51.5, 49.4, 24.9, 21.2, 19.0; HRMS (ESI) m/z: [M + H] Calcd for $\mathrm{C}_{17} \mathrm{H}_{21} \mathrm{~N}_{2} \mathrm{O}_{3}$ 301.1547; Found 301.1554.

Methyl 1-(2,6-dichlorophenyl)-6,7-dihydro-7,7-dimethyl-5-oxo-1H,5H-pyrazolo[1,2a]pyrazole-2-carboxy-late (1k). ${ }^{7}$ Prepared from AMI10 according to a literature procedure. ${ }^{7}$ ${ }^{1} \mathrm{H}$ NMR data is in agreement with the reported values. ${ }^{7}$

Methyl 7,7-dimethyl-5-oxo-1-(3,4,5-trimethoxyphenyl)-6,7-dihydro-1H,5H-pyrazolo[1,2a]pyrazole-2-carboxylate (1). Prepared according to the general procedure from AMI11. ${ }^{1} \mathrm{H}$ NMR data is in agreement with the reported values. ${ }^{11}$

Methyl 7,7-dimethyl-1-(naphthalen-2-yl)-5-oxo-6,7-dihydro-1H,5H-pyrazolo[1,2-a]pyrazole2-carboxylate (1m). Prepared according to general procedure from AMI12 (504 mg, $2.0 \mathrm{mmol}$ ) and methyl propiolate $(214 \mu \mathrm{L}, 2.4 \mathrm{mmol}) ; 500 \mathrm{mg}$ (74\% yield); yellow solid, amorphic; $v_{\max } / \mathrm{cm}^{-1}$ (ATR) 1690, 1599, 1204, 758; $\delta_{\mathrm{H}}\left(500 \mathrm{MHz} ; \mathrm{CDCl}_{3} ; \mathrm{Me}_{4} \mathrm{Si}\right) 7.87$ (s, 1H), $7.86-7.81$ $(\mathrm{m}, 3 \mathrm{H}), 7.61(\mathrm{dd}, J=8.5,1.7 \mathrm{~Hz}, 1 \mathrm{H}), 7.56(\mathrm{~d}, J=1.4 \mathrm{~Hz}, 1 \mathrm{H}), 7.50-7.44(\mathrm{~m}, 2 \mathrm{H}), 5.65(\mathrm{~d}, J=$ $1.0 \mathrm{~Hz}, 1 \mathrm{H}$ ), 3.59 (s, 3H), 2.89 (d, J = $15.8 \mathrm{~Hz}, 1 \mathrm{H}$ ), 2.42 (d, J=15.7 Hz, 1H), $1.29(\mathrm{~s}, 3 \mathrm{H}), 1.14$ (s, $3 \mathrm{H}) ; \delta_{c}\left(126 \mathrm{MHz} ; \mathrm{CDCl}_{3}\right.$; Mes $\mathrm{Si}$ ) 166.5, 164.1, 139.3, 133.2, 133.2, 129.5, 128.3, 128.1, 127.8, $126.9,126.1,126.0,125.7,116.8,64.8,64.5,51.5,49.5,25.0,19.1 ; \mathrm{HRMS}$ (ESI) m/z: [M + H] ${ }^{+}$ Calcd for $\mathrm{C}_{20} \mathrm{H}_{21} \mathrm{~N}_{2} \mathrm{O}_{3}$ 337.1547; Found 337.1547.

6-Acetyl-5-(4-methoxyphenyl)-3,3-dimethyl-2,3-dihydro-1H,5H-pyrazolo[1,2-a]pyrazol-1-one (1n). Prepared according to the general procedure from AMI3 (464 mg, $2.0 \mathrm{mmol}$ ) and 3butyn-2-one (188 $\mu \mathrm{L}, 2.4 \mathrm{mmol}$ ); $590 \mathrm{mg}$ (98\% yield); yellow solid, $\mathrm{mp} 147-149^{\circ} \mathrm{C} ; \mathrm{v}_{\mathrm{max}} / \mathrm{cm}^{-1}$ (ATR) 2970, 1722, 1652, 1234; $\delta_{\mathrm{H}}\left(500 \mathrm{MHz} ; \mathrm{CDCl}_{3} ; \mathrm{Me}_{4} \mathrm{Si}\right) 7.49$ (d, J = $\left.1.2 \mathrm{~Hz}, 1 \mathrm{H}\right), 7.38-7.32$ $(\mathrm{m}, 2 \mathrm{H}), 6.89-6.83(\mathrm{~m}, 2 \mathrm{H}), 5.48(\mathrm{~s}, 1 \mathrm{H}), 3.79(\mathrm{~s}, 3 \mathrm{H}), 2.88(\mathrm{~d}, J=15.8 \mathrm{~Hz}, 1 \mathrm{H}), 2.41(\mathrm{~d}, J=15.7$ $\mathrm{Hz}, 1 \mathrm{H}), 2.19(\mathrm{~s}, 3 \mathrm{H}), 1.20(\mathrm{~s}, 3 \mathrm{H}), 1.16(\mathrm{~s}, 3 \mathrm{H}) ; \delta_{\mathrm{c}}\left(126 \mathrm{MHz}\right.$; CDCl $\left.; \mathrm{Me}_{4} \mathrm{Si}\right)$ 193.0, 167.4, 159.1, 134.2, 129.2, 128.8, 126.6, 113.7, 64.5, 63.8, 55.2, 49.4, 26.9, 24.9, 18.9; HRMS (ESI) m/z: $[\mathrm{M}+\mathrm{H}]^{+}$Calcd for $\mathrm{C}_{17} \mathrm{H}_{21} \mathrm{~N}_{2} \mathrm{O}_{3} 301.1547$; Found 301.1543 .

Methyl $\quad\left(1 S^{*}, 6 R^{*}\right)$-6-benzyloxycarbonylamino-7,7-dimethyl-5-oxo-1-phenyl-6,7-dihydro$1 H, 5 H$-pyrazolo[1,2-a]pyrazole-2-carboxylate (10). Prepared according to the general procedure from AMI13. ${ }^{1} \mathrm{H}$ NMR data is in agreement with the reported values. ${ }^{12}$

Methyl 7,7-dimethyl-5-oxo-1-(1-tosyl-1H-indol-3-yl)-6,7-dihydro-1H,5H-pyrazolo[1,2a]pyrazole-2-carboxylate (1p). Prepared according to the general procedure from AMI14 (790 $\mathrm{mg}, 2.0 \mathrm{mmol})$ and methyl propiolate $(214 \mu \mathrm{L}, 2.4 \mathrm{mmol}) ; 612 \mathrm{mg}(64 \%$ yield); yellow solid,

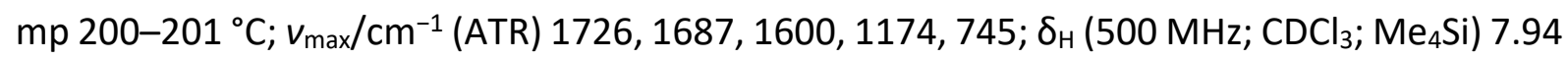
(d, $J=8.3 \mathrm{~Hz}, 1 \mathrm{H}), 7.72(\mathrm{~d}, J=8.1 \mathrm{~Hz}, 2 \mathrm{H}), 7.68(\mathrm{~d}, J=7.9 \mathrm{~Hz}, 1 \mathrm{H}), 7.65(\mathrm{~s}, 1 \mathrm{H}), 7.56(\mathrm{~s}, 1 \mathrm{H}), 7.29$ $(\mathrm{t}, J=7.7 \mathrm{~Hz}, 1 \mathrm{H}), 7.24-7.14(\mathrm{~m}, 3 \mathrm{H}), 5.74(\mathrm{~s}, 1 \mathrm{H}), 3.54(\mathrm{~s}, 3 \mathrm{H}), 2.85(\mathrm{~d}, J=15.8 \mathrm{~Hz}, 1 \mathrm{H}), 2.41$ (d, $J=15.8 \mathrm{~Hz}, 1 \mathrm{H}), 2.32(\mathrm{~s}, 3 \mathrm{H}), 1.26(\mathrm{~s}, 3 \mathrm{H}), 1.10(\mathrm{~s}, 3 \mathrm{H}) ; \delta_{\mathrm{C}}\left(126 \mathrm{MHz} ; \mathrm{CDCl}_{3} ; \mathrm{Me}_{4} \mathrm{Si}\right)$ 166.5, 163.8, 144.9, 135.7, 135.2, 129.8 (2C), 129.1, 126.8, 125.0, 124.8, 123.2, 122.9, 120.9, 115.4, 113.8, 64.4, 57.6, 51.4, 49.4, 24.9, 21.6, 18.9; HRMS (ESI) m/z: [M+ H] Calcd for $\mathrm{C}_{25} \mathrm{H}_{26} \mathrm{~N}_{3} \mathrm{O}_{5} \mathrm{~S}$ 480.1588; Found 480.1578 . 
tert-Butyl ((S)-1-((S)-1-(4-chlorophenyl)-7,7-dimethyl-5-oxo-6,7-dihydro-1H,5H-pyrazolo[1,2a]pyrazol-2-yl)-1-oxopropan-2-yl)carbamate (1q) and tert-butyl ((S)-1-((R)-1-(4-chlorophenyl)7,7-dimethyl-5-oxo-6,7-dihydro-1H,5H-pyrazolo[1,2-a]pyrazol-2-yl)-1-oxopropan-2-

$\mathrm{yl})$ carbamate $\left(\mathbf{1} \mathbf{q}^{\prime}\right)$. Prepared according to the general procedure from AMI1 (558.2 mg, 2.36 mmol) and tert-butyl (S)-(3-oxopent-4-yn-2-yl)carbamate $(560 \mathrm{mg}, 2.83 \mathrm{mmol}$, prepared according to the literature); ${ }^{13}{ }^{1} \mathrm{H}$ NMR analysis of the crude reaction mixture revealed that $\mathbf{1 q}$ and $\mathbf{1} \mathbf{q}^{\prime}$ are formed in a 1.8:1 ratio. After work-up the crude product weas triturated with diethyl ether. Product 1q was collected by filtration. Stereochemistry of the isomers was assigned by optical rotation. ${ }^{11} 240 \mathrm{mg}$ (24\% yield); yellow solid, $\mathrm{mp} 204-205^{\circ} \mathrm{C} ;[\alpha]_{589}^{25}+587$ (c 0.50, MeOH); $v_{\max } / \mathrm{cm}^{-1}$ (ATR) 3306, 3077, 2971, 1721, 1702, 1647, 1583, 1237, 1161, 1014; $\delta_{\mathrm{H}}\left(500 \mathrm{MHz} ; \mathrm{CDCl}_{3} ; \mathrm{Me}_{4} \mathrm{Si}\right) 7.64(\mathrm{~s}, 1 \mathrm{H}), 7.39(\mathrm{~d}, J=8.2 \mathrm{~Hz}, 2 \mathrm{H}), 7.30$ (d, J = 8.2 Hz, 2H), 5.55 (s, $1 \mathrm{H}), 5.16(\mathrm{~d}, J=7.2 \mathrm{~Hz}, 1 \mathrm{H}), 4.71-4.57(\mathrm{~m}, 1 \mathrm{H}), 2.87$ (d, J=15.9 Hz, 1H), $2.45(\mathrm{~d}, J=15.9 \mathrm{~Hz}$, 1H), $1.42(\mathrm{~s}, 9 \mathrm{H}), 1.24-1.10(\mathrm{~m}, 9 \mathrm{H}) ; \delta_{\mathrm{c}}\left(126 \mathrm{MHz}\right.$; $\mathrm{CDCl}_{3}$; Me $\mathrm{MSi}_{4}$ 194.8, 166.8, 155.0, 140.4, 133.6, 129.3, 129.0, 128.6, 122.8, 79.8, 64.4, 64.0, 51.7, 49.3, 28.3, 25.0, 19.6, 19.0; HRMS (ESI) $\mathrm{m} / \mathrm{z}:[\mathrm{M}+\mathrm{H}]^{+}$Calcd for $\mathrm{C}_{22} \mathrm{H}_{29} \mathrm{ClN}_{3} \mathrm{O}_{4} 434.1841$; Found 434.1833 .

Methyl 7,7-dimethyl-1-(1-methyl-1H-pyrrol-2-yl)-5-oxo-6,7-dihydro-1H,5H-pyrazolo[1,2a]pyrazole-2-carboxylate (1r). Prepared according to the general procedure from AMI15 (410 $\mathrm{mg}, 2.0 \mathrm{mmol})$ and methyl propiolate $(214 \mu \mathrm{L}, 2.4 \mathrm{mmol})$. The reaction mixture was filtered and evaporated in vacuo. Product $1 \mathrm{r}$ was additionally purified by CC (EA/PE, 1:2). $408 \mathrm{mg}$ (71\% yield); orange solid, $\mathrm{mp} 98-100{ }^{\circ} \mathrm{C} ; v_{\max } / \mathrm{cm}^{-1}$ (ATR) 2967, 1694, 1597, 1199; $\delta_{\mathrm{H}}(500 \mathrm{MHz}$; $\mathrm{CDCl}_{3}$; $\left.\mathrm{Me}_{4} \mathrm{Si}\right) 7.48(\mathrm{~d}, J=1.6 \mathrm{~Hz}, 1 \mathrm{H}), 6.58-6.53(\mathrm{~m}, 1 \mathrm{H}), 6.12(\mathrm{dd}, J=3.5,1.9 \mathrm{~Hz}, 1 \mathrm{H}), 6.06-$ $6.02(\mathrm{~m}, 1 \mathrm{H}), 5.64(\mathrm{~d}, J=1.4 \mathrm{~Hz}, 1 \mathrm{H}), 3.69(\mathrm{~s}, 3 \mathrm{H}), 3.65(\mathrm{~s}, 3 \mathrm{H}), 2.84(\mathrm{~d}, J=15.8 \mathrm{~Hz}, 1 \mathrm{H}), 2.42(\mathrm{~d}$, $J=15.8 \mathrm{~Hz}, 1 \mathrm{H}), 1.25(\mathrm{~s}, J=6.5 \mathrm{~Hz}, 3 \mathrm{H}), 1.17(\mathrm{~s}, 3 \mathrm{H}) ; \delta_{\mathrm{c}}\left(126 \mathrm{MHz}\right.$; CDCl$\left.; \mathrm{Me}_{4} \mathrm{Si}\right)$ 165.9, 163.9, $129.9,129.1,123.9,115.5,109.8,106.6,63.9,58.4,51.6,49.7,34.7,25.1,19.1$; HRMS (ESI) $\mathrm{m} / \mathrm{z}:[\mathrm{M}+\mathrm{H}]^{+}$Calcd for $\mathrm{C}_{15} \mathrm{H}_{20} \mathrm{~N}_{3} \mathrm{O}_{3}$ 290.1499; Found 290.1496.

\section{Optimization studies for the synthesis of pyrazoles 2}

Under irradiation alone, $\mathbf{1 a}$ could be converted to the substituted pyrazole $\mathbf{2 a}$. While the reaction took place in all solvents tested, namely $\mathrm{DCM}$, THF, acetone, $\mathrm{MeOH}$, toluene, $\mathrm{MeCN}$ DMF and ethyl acetate, DCM gave the optimal yield. Optimization studies revealed that increased reaction temperature resulted in side reactions. Varying the light source from $400 \mathrm{~nm}$ to $450 \mathrm{~nm}$ proved less effective (Table SI1). 
Table SI1: Optimization studies for the synthesis of $\mathbf{2 a .}$

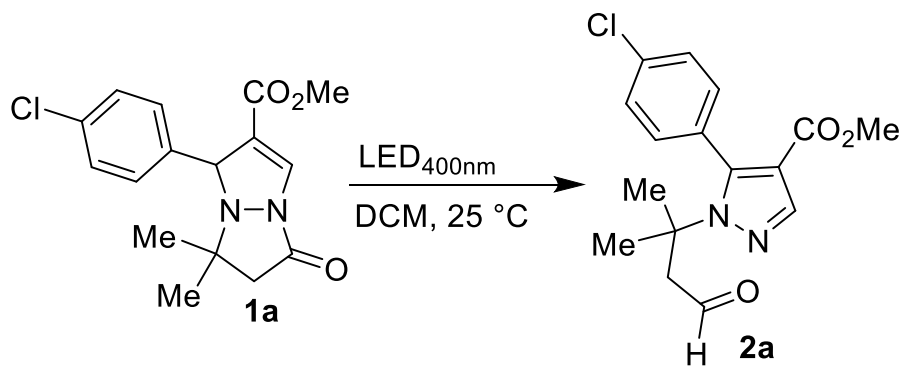

$\begin{array}{lll}\text { entry } & \text { deviation from standard conditions } & \text { yield } 2 \mathrm{a}(\%)^{\mathrm{a}} \\ 1 & \text { no deviation } & 82(78)^{\mathrm{b}} \\ 2 & \text { THF } & 66 \\ 3 & \text { acetone } & 50 \\ 4 & \mathrm{MeOH} & 31 \\ 5 & \text { toluene } & 28 \\ 6 & \text { MeCN } & 49 \\ 7 & \text { DMF } & 40 \\ 8 & \text { ethyl acetate } & 49 \\ 9 & \text { DCE, } 50{ }^{\circ} \mathrm{C}, 12 \mathrm{~h} & 62 \\ 10 & \text { DCE, } 50{ }^{\circ} \mathrm{C} & 52 \\ 11 & \text { LED }_{450 \mathrm{~nm}} & 28\end{array}$

Standard reaction conditions: 1a $(0.5 \mathrm{mmol}), \mathrm{DCM}$ (anhydrous, degassed, $2.5 \mathrm{~mL}$ ), LED $\mathrm{L}_{400 \mathrm{~nm}}$, $25{ }^{\circ} \mathrm{C}$, under $\mathrm{N}_{2}, 24 \mathrm{~h}$. ${ }^{\mathrm{a} 1} \mathrm{H}$ NMR yields were determined with 1,3,5-trimethoxybenzene as an internal standard. ${ }^{b}$ Isolated yield of pure product.

\section{General procedure for the synthesis of aldehydes $\mathbf{2}$ and diazepines $\mathbf{3}$}

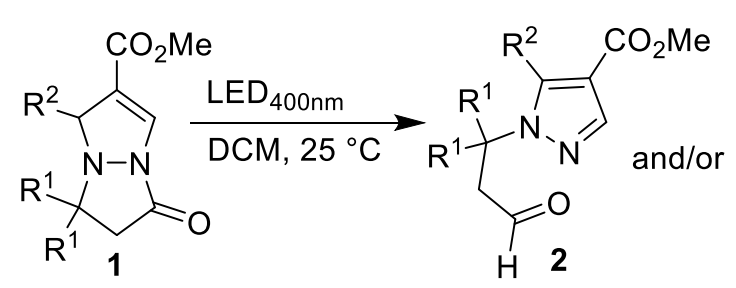<smiles>[R][R]C1([R])C=CC(C(C)=O)=CN2C(=O)CC([R])([R])N21</smiles>

Scheme SI2: Synthesis of compounds 2 and $\mathbf{3}$.

A dried $8 \mathrm{~mL}$ vial was charged with $1(0.5 \mathrm{mmol})$ and dry DCM $(2.5 \mathrm{~mL})$ and sealed off with a screw cap with a septum. The resulting solution was degassed via needle by three freezepump-thaw cycles and irradiated with $\mathrm{LED}_{400 \mathrm{~nm}}$ under nitrogen atmosphere for $24-48 \mathrm{~h}$ at 25 ${ }^{\circ} \mathrm{C}$. Products $\mathbf{2}$ and $\mathbf{3}$ were obtained by evaporation of the solvent and purification by CC (EA/PE). 


\section{Characterization data of novel compounds $\mathbf{2}$ and $\mathbf{3}$}

Methyl 5-(4-chlorophenyl)-1-(2-methyl-4-oxobutan-2-yl)-1H-pyrazole-4-carboxylate (2a). Prepared according to the general procedure from 1a (161 mg, $0.5 \mathrm{mmol}) ; \mathrm{CC}$ (EA/PE, 1:2); $126 \mathrm{mg}$ (78\% yield); colorless oil; $v_{\mathrm{max}} / \mathrm{cm}^{-1}$ (ATR) $2950,1715,1546 ; \delta_{\mathrm{H}}\left(500 \mathrm{MHz} ; \mathrm{CDCl}_{3} ; \mathrm{Me}_{4} \mathrm{Si}\right)$ $9.71(\mathrm{t}, J=1.9 \mathrm{~Hz}, 1 \mathrm{H}), 7.90(\mathrm{~s}, 1 \mathrm{H}), 7.44(\mathrm{~d}, J=8.4 \mathrm{~Hz}, 2 \mathrm{H}), 7.28(\mathrm{~d}, J=8.5 \mathrm{~Hz}, 2 \mathrm{H}), 3.63(\mathrm{~s}, 3 \mathrm{H})$, $2.94(\mathrm{~d}, J=1.9 \mathrm{~Hz}, 2 \mathrm{H}), 1.48(\mathrm{~s}, 6 \mathrm{H}) ; \delta_{\mathrm{C}}\left(126 \mathrm{MHz} ; \mathrm{CDCl}_{3} ; \mathrm{Me}_{4} \mathrm{Si}\right)$ 199.2, 163.1, 144.6, 139.4, 135.5, 131.6, 129.9, 128.5, 115.1, 63.4, 55.1, 51.1, 29.6; HRMS (ESI) m/z: [M + H] ${ }^{+}$Calcd for $\mathrm{C}_{16} \mathrm{H}_{18} \mathrm{ClN}_{2} \mathrm{O}_{3} 321.1000$; Found 321.1000.

Methyl 1-(2-methyl-4-oxobutan-2-yl)-5-phenyl-1H-pyrazole-4-carboxylate (2b). Prepared according to the general procedure from $\mathbf{1 b}$ (143 mg, $0.5 \mathrm{mmol}) ; C C$ (EA/PE, 1:2); $123 \mathrm{mg}$ (86\% yield); white solid; $\mathrm{mp} 86-87^{\circ} \mathrm{C} ; v_{\max } / \mathrm{cm}^{-1}$ (ATR) $2984,1720,1713,1546 ; \delta_{\mathrm{H}}\left(500 \mathrm{MHz} ; \mathrm{CDCl}_{3}\right.$; $\left.\mathrm{Me}_{4} \mathrm{Si}\right) 9.71(\mathrm{t}, J=2.1 \mathrm{~Hz}, 1 \mathrm{H}), 7.91(\mathrm{~s}, 1 \mathrm{H}), 7.50-7.42(\mathrm{~m}, 3 \mathrm{H}), 7.36-7.30(\mathrm{~m}, 2 \mathrm{H}), 3.61(\mathrm{~s}$, $3 \mathrm{H}), 2.91(\mathrm{~d}, J=2.1 \mathrm{~Hz}, 2 \mathrm{H}), 1.47(\mathrm{~s}, 6 \mathrm{H}) ; \delta_{\mathrm{c}}\left(126 \mathrm{MHz}\right.$; CDCl $\left.; \mathrm{Me}_{4} \mathrm{Si}\right)$ 199.5, 163.2, 145.9, 139.3, 131.3, 130.2, 129.2, 128.0, 114.9, 63.2, 55.1, 51.0, 29.5; HRMS (ESI) m/z: [M + H] ${ }^{+}$Calcd for $\mathrm{C}_{16} \mathrm{H}_{19} \mathrm{~N}_{2} \mathrm{O}_{3}$ 287.1390; Found 287.1387.

Methyl 5-(4-methoxyphenyl)-1-(2-methyl-4-oxobutan-2-yl)-1H-pyrazole-4-carboxylate (2c). Prepared according to the general procedure from 1c (158 mg, $0.5 \mathrm{mmol}) ; C C$ (EA/PE, 1:1); $134 \mathrm{mg}$ ( $85 \%$ yield); white solid; $\mathrm{mp} 122-123{ }^{\circ} \mathrm{C} ; v_{\max } / \mathrm{cm}^{-1}$ (ATR) 2841, 1730, 1715, 1612, 1553; $\delta_{\mathrm{H}}\left(500 \mathrm{MHz} ; \mathrm{CDCl}_{3} ; \mathrm{Me}_{4} \mathrm{Si}\right) 9.70(\mathrm{t}, J=2.0 \mathrm{~Hz}, 1 \mathrm{H}), 7.89(\mathrm{~s}, 1 \mathrm{H}), 7.23(\mathrm{~d}, J=8.7 \mathrm{~Hz}, 2 \mathrm{H})$, $6.98(\mathrm{~d}, J=8.7 \mathrm{~Hz}, 2 \mathrm{H}), 3.86(\mathrm{~s}, 3 \mathrm{H}), 3.63(\mathrm{~s}, 3 \mathrm{H}), 2.91(\mathrm{~d}, J=1.9 \mathrm{~Hz}, 2 \mathrm{H}), 1.49(\mathrm{~s}, 6 \mathrm{H}) ; \delta_{\mathrm{c}}(126$ $\mathrm{MHz} ; \mathrm{CDCl}_{3}$; Me $\left.4 \mathrm{Si}\right)$ 199.6, 163.3, 160.1, 145.9, 139.2, 131.4, 123.0, 114.9, 113.6, 63.1, 55.2, 55.1, 51.0, 29.5; HRMS (ESI) m/z: [M+ H] Calcd for $\mathrm{C}_{17} \mathrm{H}_{21} \mathrm{~N}_{2} \mathrm{O}_{4}$ 317,1496; Found 317.1491.

Methyl 5-(4-cyanophenyl)-1-(2-methyl-4-oxobutan-2-yl)-1H-pyrazole-4-carboxylate (2d). Prepared according to the general procedure from 1d (156 mg, $0.5 \mathrm{mmol}) ; C C(E A / P E, 1: 1)$; $137 \mathrm{mg}$ (88\% yield); white solid; $\mathrm{mp} 133-134{ }^{\circ} \mathrm{C} ; v_{\max } / \mathrm{cm}^{-1}$ (ATR) 2986, 2227, 1712, 1553; $\delta_{H}$ $\left(500 \mathrm{MHz} ; \mathrm{CDCl}_{3}\right.$; $\left.\mathrm{Me}_{4} \mathrm{Si}\right) 9.72(\mathrm{t}, J=1.6 \mathrm{~Hz}, 1 \mathrm{H}), 7.91(\mathrm{~s}, 1 \mathrm{H}), 7.76(\mathrm{~d}, J=8.3 \mathrm{~Hz}, 2 \mathrm{H}), 7.51(\mathrm{~d}, J=$ $8.3 \mathrm{~Hz}, 2 \mathrm{H}$ ), $3.63(\mathrm{~s}, 3 \mathrm{H}), 2.99$ (d, $J=1.5 \mathrm{~Hz}, 2 \mathrm{H}), 1.46(\mathrm{~s}, 6 \mathrm{H}) ; \delta_{c}\left(126 \mathrm{MHz} ; \mathrm{CDCl}_{3} ; \mathrm{Me}_{4} \mathrm{Si}\right)$ 198.9, 162.9, 143.7, 139.5, 136.6, 131.8, 131.2, 118.2, 115.1, 113.3, 63.5, 55.0, 51.2, 29.7; HRMS (ESI) $\mathrm{m} / \mathrm{z}:[\mathrm{M}+\mathrm{H}]^{+}$Calcd for $\mathrm{C}_{17} \mathrm{H}_{18} \mathrm{~N}_{3} \mathrm{O}_{3}$ 312.1343; Found 312.1341.

Methyl 5-(furan-2-yl)-1-(2-methyl-4-oxobutan-2-yl)-1H-pyrazole-4-carboxylate (2e). Prepared according to the general procedure from 1e (138 mg, $0.5 \mathrm{mmol})$; CC (EA/PE, 1:2); $57 \mathrm{mg}$ (41\% yield); colorless oil; $v_{\max } / \mathrm{cm}^{-1}(\mathrm{ATR}) 2951,1715,1537 ; \delta_{\mathrm{H}}\left(500 \mathrm{MHz} ; \mathrm{CDCl}_{3} ; \mathrm{Me}_{4} \mathrm{Si}\right) 9.68$ (t, $J=$ $2.1 \mathrm{~Hz}, 1 \mathrm{H}), 7.92(\mathrm{~s}, 1 \mathrm{H}), 7.62(\mathrm{~d}, J=1.7 \mathrm{~Hz}, 1 \mathrm{H}), 6.62(\mathrm{~d}, J=3.3 \mathrm{~Hz}, 1 \mathrm{H}), 6.58$ (dd, $J=3.3,1.9$ $\mathrm{Hz}, 1 \mathrm{H}), 3.72(\mathrm{~s}, 3 \mathrm{H}), 2.92(\mathrm{~d}, J=2.1 \mathrm{~Hz}, 2 \mathrm{H}), 1.54(\mathrm{~s}, 6 \mathrm{H}) ; \delta_{c}\left(126 \mathrm{MHz} ; \mathrm{CDCl}_{3} ; \mathrm{Me}_{4} \mathrm{Si}\right)$ 199.5, 162.8, 143.3, 140.7, 139.3, 134.6, 117.0, 114.2, 111.5, 63.0, 54.6, 51.4, 28.4; HRMS (ESI) m/z: $[\mathrm{M}+\mathrm{H}]^{+}$Calcd for $\mathrm{C}_{14} \mathrm{H}_{17} \mathrm{~N}_{2} \mathrm{O}_{4}$ 277.1183; Found 277.1180.

Methyl 5-methyl-1-(2-methyl-4-oxobutan-2-yl)-1H-pyrazole-4-carboxylate (2f). Prepared according to the general procedure from $1 \mathrm{ff}(112 \mathrm{mg}, 0.5 \mathrm{mmol}) ; \mathrm{CC}$ (EA/PE, 1:2); $86 \mathrm{mg}$ (77\% yield); colorless oil; $v_{\max } / \mathrm{cm}^{-1}$ (ATR) 2951, 1707, 1551; $\delta_{\mathrm{H}}\left(500 \mathrm{MHz} ; \mathrm{CDCl}_{3} ; \mathrm{Me}_{4} \mathrm{Si}\right) 9.73$ (t, $J=$ $2.0 \mathrm{~Hz}, 1 \mathrm{H}), 7.75(\mathrm{~s}, 1 \mathrm{H}), 3.81(\mathrm{~s}, 3 \mathrm{H}), 3.01(\mathrm{~d}, J=2.0 \mathrm{~Hz}, 2 \mathrm{H}), 2.77(\mathrm{~s}, 3 \mathrm{H}), 1.76(\mathrm{~s}, 6 \mathrm{H}) ; \delta_{c}(126$ 
$\mathrm{MHz} ; \mathrm{CDCl}_{3}$; $\mathrm{Me}_{4} \mathrm{Si}$ ) 199.4, 164.3, 142.8, 139.1, 113.3, 62.0, 54.6, 51.0, 28.2, 12.8; HRMS (ESI) $\mathrm{m} / \mathrm{z}:[\mathrm{M}+\mathrm{H}]^{+}$Calcd for $\mathrm{C}_{11} \mathrm{H}_{17} \mathrm{~N}_{2} \mathrm{O}_{3}$ 225.1234; Found 225.1233.

Methyl 1-(3-oxopropyl)-5-phenyl-1H-pyrazole-4-carboxylate (2g). Prepared according to the general procedure from $1 \mathrm{~g}$ (129 mg, $0.5 \mathrm{mmol}) ; \mathrm{CC}$ (EA/PE, 1:1); $92 \mathrm{mg}$ (71\% yield); white solid;

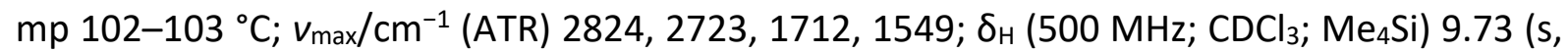
$1 \mathrm{H}), 7.98(\mathrm{~s}, 1 \mathrm{H}), 7.53-7.44(\mathrm{~m}, 3 \mathrm{H}), 7.42-7.34(\mathrm{~m}, 2 \mathrm{H}), 4.27(\mathrm{t}, J=6.8 \mathrm{~Hz}, 2 \mathrm{H}), 3.68(\mathrm{~s}, 3 \mathrm{H})$, 3.00 (td, $J=6.7,0.6 \mathrm{~Hz}, 2 \mathrm{H}) ; \delta_{c}\left(126 \mathrm{MHz} ; \mathrm{CDCl}_{3} ; \mathrm{Me}_{4} \mathrm{Si}\right)$ 198.9, 163.3, 146.2, 141.4, 129.9, 129.6, 128.6, 128.5, 112.6, 51.1, 43.0, 43.0; HRMS (ESI) m/z: $[\mathrm{M}+\mathrm{H}]^{+}$Calcd for $\mathrm{C}_{14} \mathrm{H}_{15} \mathrm{~N}_{2} \mathrm{O}_{3}$ 259.1077; Found 259.1078.

Methyl (E)-1-(2-methyl-4-oxobutan-2-yl)-5-(prop-1-en-1-yl)-1H-pyrazole-4-carboxylate (2h) and methyl 3,3-dimethyl-1-oxo-5-methyl-2,3-dihydro-1H,5H-pyrazolo[1,2-a][1,2]diazepine-8carboxylate (3h). Prepared according to the general procedure from $\mathbf{1 h}$ (125 $\mathrm{mg}, 0.5 \mathrm{mmol}$ ). Purification by CC (EA/PE, 1:1) gave products $\mathbf{2 h}$ and $\mathbf{3 h}$. $\mathbf{2 h}$; $51 \mathrm{mg}$ ( $41 \%$ yield); colorless oil; $v_{\text {max }} / \mathrm{cm}^{-1}$ (ATR) 2949, 1716, 1535; $\delta_{\mathrm{H}}\left(500 \mathrm{MHz} ; \mathrm{CDCl}_{3}\right.$; $\left.\mathrm{Me}_{4} \mathrm{Si}\right) 9.69(\mathrm{t}, J=2.1 \mathrm{~Hz}, 1 \mathrm{H}), 7.78(\mathrm{~s}$, $1 \mathrm{H}), 6.36(\mathrm{dq}, J=15.8,1.6 \mathrm{~Hz}, 1 \mathrm{H}), 6.21(\mathrm{dq}, J=15.8,6.6 \mathrm{~Hz}, 1 \mathrm{H}), 3.77(\mathrm{~s}, 3 \mathrm{H}), 2.98(\mathrm{~d}, J=2.1 \mathrm{~Hz}$, 2H), 1.97 (dd, $J=6.6,1.7 \mathrm{~Hz}, 3 \mathrm{H}), 1.73(\mathrm{~s}, 6 \mathrm{H}) ; \delta_{\mathrm{c}}\left(126 \mathrm{MHz}\right.$; CDCl $\left.; \mathrm{Me}_{4} \mathrm{Si}\right)$ 199.6, 163.8, 143.5, 139.7, 137.4, 117.8, 113.4, 62.3, 54.5, 51.1, 28.7, 19.0; HRMS (ESI) m/z: [M + H] ${ }^{+}$Calcd for $\mathrm{C}_{13} \mathrm{H}_{19} \mathrm{~N}_{2} \mathrm{O}_{3}$ 251.1390; Found 251.1387. 3h; $31 \mathrm{mg}$ (25\% yield); colorless oil; $v_{\max } / \mathrm{cm}^{-1}$ (ATR) 2973, 1702, 1640, 1604, 1197; $\delta_{\mathrm{H}}\left(500 \mathrm{MHz} ; \mathrm{CDCl}_{3}\right.$; Me $\left.4 \mathrm{Si}\right) 8.16$ (d, J = $\left.1.3 \mathrm{~Hz}, 1 \mathrm{H}\right), 6.47$ (dd, J $=11.8,1.4 \mathrm{~Hz}, 1 \mathrm{H}), 6.02(\mathrm{dd}, J=11.8,7.4 \mathrm{~Hz}, 1 \mathrm{H}), 4.14(\mathrm{p}, J=6.7 \mathrm{~Hz}, 1 \mathrm{H}), 3.78(\mathrm{~s}, 3 \mathrm{H}), 2.73(\mathrm{~d}$, $J=17.0 \mathrm{~Hz}, 1 \mathrm{H}), 2.29$ (d, $J=17.1 \mathrm{~Hz}, 1 \mathrm{H}), 1.45(\mathrm{~s}, 3 \mathrm{H}), 1.26(\mathrm{~s}, 3 \mathrm{H}), 1.12(\mathrm{~d}, J=6.5 \mathrm{~Hz}, 3 \mathrm{H}) ; \delta_{c}$ (126 MHz; $\mathrm{CDCl}_{3} ; \mathrm{Me}_{4} \mathrm{Si}$ ) 172.1, 167.7, 134.6, 131.7, 121.4, 109.8, 59.9, 58.3, 52.0, 43.4, 31.1, 23.9, 15.7; HRMS (ESI) m/z: [M + H] $]^{+}$Calcd for $\mathrm{C}_{13} \mathrm{H}_{19} \mathrm{~N}_{2} \mathrm{O}_{3}$ 251.1390; Found 251.1388.

Methyl 3,3-dimethyl-1-oxo-5-phenyl-2,3-dihydro-1H,5H-pyrazolo[1,2-a][1,2]diazepine-8carboxylate (3i) Prepared according to the general procedure from 1i (156 mg, $0.5 \mathrm{mmol}$ ); $\mathrm{LED}_{450 \mathrm{~nm}}$ were used; CC (EA/PE, 1:2); $117 \mathrm{mg}$ (75\% yield); pale yellow solid; $\mathrm{mp} 151-152{ }^{\circ} \mathrm{C}$; $v_{\max } / \mathrm{cm}^{-1}$ (ATR) 2973, 1704, 1628, 1601; $\delta_{\mathrm{H}}\left(500 \mathrm{MHz} ; \mathrm{CDCl}_{3} ; \mathrm{Me}_{4} \mathrm{Si}\right) 8.28$ (d, $\left.J=1.1 \mathrm{~Hz}, 1 \mathrm{H}\right)$, $7.32-7.27(\mathrm{~m}, 3 \mathrm{H}), 7.17-7.12(\mathrm{~m}, 2 \mathrm{H}), 6.60(\mathrm{dd}, J=12.0,0.5 \mathrm{~Hz}, 1 \mathrm{H}), 6.11$ (dd, $J=12.0,7.2 \mathrm{~Hz}$, $1 \mathrm{H}), 5.06(\mathrm{~d}, J=7.2 \mathrm{~Hz}, 1 \mathrm{H}), 3.84(\mathrm{~s}, 3 \mathrm{H}), 1.86(\mathrm{~d}, J=16.5 \mathrm{~Hz}, 1 \mathrm{H}), 1.65(\mathrm{~d}, J=16.7 \mathrm{~Hz}, 1 \mathrm{H}), 1.58$ (s, 3H), 1.22 (s, 3H); $\delta_{c}\left(126 \mathrm{MHz} ; \mathrm{CDCl}_{3}\right.$; Me $\left.{ }_{4} \mathrm{Si}\right)$ 173.3, 167.6, 139.5, 133.0, 132.9, 129.4, 128.8, 128.5, 120.7, 111.5, 67.4, 61.8, 52.1, 42.1, 30.5, 23.8; HRMS (ESI) m/z: $[\mathrm{M}+\mathrm{H}]^{+}$Calcd for $\mathrm{C}_{18} \mathrm{H}_{21} \mathrm{~N}_{2} \mathrm{O}_{3} 313.1547$; Found 313.1543. 


\section{Optimization studies for the synthesis of pyrazoles 4}

In the presence of diethyl bromomalonate, 1a could be converted to the N1-acryloyl substituted pyrazole 4a. Optimization studies revealed that solvents such as $\mathrm{MeCN}$, toluene, and $\mathrm{CHCl}_{3}$ were less suitable, resulting in lower conversions. Considering the fact that an equivalent of $\mathrm{HBr}$ is generated in this reaction, a series of bases were subsequently introduced to promote the conversion. The reaction carried out without a base proved to be inefficient, while pyridine allowed the formation of $4 \mathrm{a}$ in a low $38 \%$ yield. Furthermore, when inorganic bases $\left(\mathrm{Cs}_{2} \mathrm{CO}_{3}, \mathrm{~K}_{2} \mathrm{CO}_{3}\right.$ and $\left.\mathrm{KH}_{2} \mathrm{PO}_{4}\right)$ were examined, $\mathrm{Cs}_{2} \mathrm{CO}_{3}$ proved to be the most effective as it gave the desired product $4 \mathrm{a}$ in reasonable $50-60 \%$ yield. Varying the light source above $450 \mathrm{~nm}$ proved less effective, as no product formation was observed at $510 \mathrm{~nm}$. Moreover, for the reaction to be productive the exclusion of oxygen was necessary, since no product was detected in the case when the reaction was run under air atmosphere. It should be mentioned that the presence of moisture decreased the yield of 4 a substantially (Table SI2, entry 10).

Table SI2: Optimization studies for the synthesis of $4 a$.
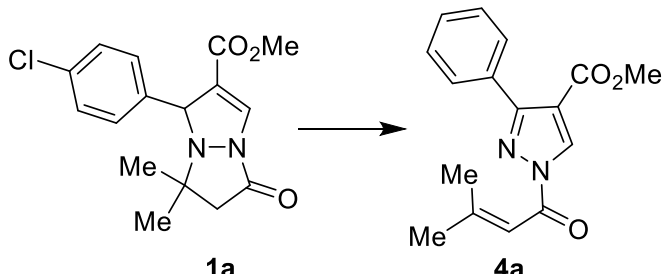

1a

4a

\begin{tabular}{lll}
\hline entry & deviation from standard conditions & yield 4a (\%) \\
\hline 1 & MeCN, $\mathrm{Cs}_{2} \mathrm{CO}_{3}$ (1.0 equiv) & 54 \\
2 & toluene, $\mathrm{Cs}_{2} \mathrm{CO}_{3}$ (1.0 equiv) & 50 \\
3 & $\mathrm{CHCl}_{3}, \mathrm{Cs}_{2} \mathrm{CO}_{3}$ (1.0 equiv) & 47 \\
4 & $\mathrm{Cs}_{2} \mathrm{CO}_{3}$ (1.0 equiv) & 64 \\
5 & $\mathrm{KH}_{2} \mathrm{PO}_{4}$ or $\mathrm{K}_{2} \mathrm{CO}_{3}$ (1.0 equiv) & 0 \\
6 & no deviation & $85(78)^{\mathrm{b}}[80]^{\mathrm{c}}$ \\
7 & pyridine (1.5 equiv) & 38 \\
8 & no base & 0 \\
9 & dietyl bromomalonate (1.5 equiv) & 55 \\
10 & $\mathrm{H} O$ & $24^{\mathrm{d}}$ \\
11 & air atmosphere & 0 \\
12 & 400 nm, (510 nm) & $75(0)$ \\
13 & no light & 0 \\
\hline
\end{tabular}

Standard reaction conditions: 1 a $(0.5 \mathrm{mmol})$, diethyl bromomalonate (2.0 equiv), 2,6-lutidine (1.5 equiv), DCM (anhydrous, degassed, $2.5 \mathrm{~mL}$ ), LED $450 \mathrm{~nm}, 25{ }^{\circ} \mathrm{C}$, under $\mathrm{N}_{2}, 18$ h. ${ }^{\text {a1 }} \mathrm{H}$ NMR yields were determined with 1,3,5-trimethoxybenzene as an internal standard. bIsolated yield of pure product. ${ }^{\mathrm{c}} 3.3 \mathrm{mmol}$ scale yield. ${ }^{\mathrm{d}} \mathbf{5} \mathrm{a}$ is present as the major product. 


\section{General procedure for the synthesis of pyrazoles 4}

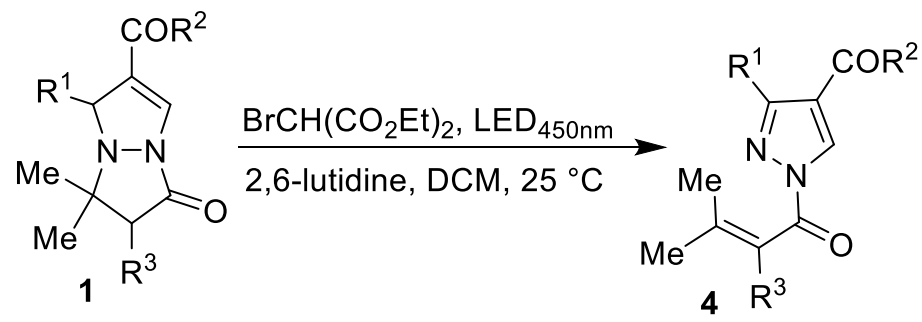

Scheme SI3: Synthesis of compounds 4.

A dried $8 \mathrm{~mL}$ vial was charged with $1(0.5 \mathrm{mmol})$ and dry DCM $(2.5 \mathrm{~mL})$ and sealed off with a screw cap with a septum. The resulting solution was degassed via needle by three freezepump-thaw cycles. Diethyl bromomalonate ( $185 \mu \mathrm{L}, 92 \%$ purity, 2.0 equiv) and 2,6-lutidine ( $87 \mu \mathrm{L}, 1.5$ equiv) were added. The solution was irradiated with a blue $\mathrm{LED}_{450 \mathrm{~nm}}$ under nitrogen atmosphere for $18 \mathrm{~h}$ at $25^{\circ} \mathrm{C}$. Products 4 were isolated by CC or CC (EA/PE) and dried under vacuum. In the optimization studies, inorganic bases were added prior to degassing.

\section{Characterization data of novel compounds 4}

Methyl 3-(4-chlorophenyl)-1-(3-methylbut-2-enoyl)-1H-pyrazole-4-carboxylate (4a). Prepared according to the general procedure from 1a (161 mg, $0.5 \mathrm{mmol}$ ); CC (EA/PE, 1:6); $126 \mathrm{mg}$ (79\% yield); white solid; $\mathrm{mp} 119-121{ }^{\circ} \mathrm{C} ; v_{\max } / \mathrm{cm}^{-1}$ (ATR) $1729,1717,1625,1147,1133 ; \delta_{H}(500$ $\left.\mathrm{MHz} ; \mathrm{CDCl}_{3} ; \mathrm{Me}_{4} \mathrm{Si}\right) 8.84(\mathrm{~s}, 1 \mathrm{H}), 7.83-7.76(\mathrm{~m}, 2 \mathrm{H}), 7.46-7.38(\mathrm{~m}, 2 \mathrm{H}), 7.18-7.14(\mathrm{~m}, 1 \mathrm{H})$, $3.83(\mathrm{~s}, 3 \mathrm{H}), 2.36(\mathrm{~d}, J=1.1 \mathrm{~Hz}, 3 \mathrm{H}), 2.11(\mathrm{~d}, J=1.2 \mathrm{~Hz}, 3 \mathrm{H}) ; \delta_{\mathrm{C}}\left(126 \mathrm{MHz} ; \mathrm{CDCl}_{3} ; \mathrm{Me}_{4} \mathrm{Si}\right)$ 165.7, $162.9,162.2$, 153.7, 135.3, 134.2, 130.7, 129.8, 128.3, 114.8, 113.2, 51.8, 28.7, 21.7; HRMS (ESI) $\mathrm{m} / \mathrm{z}:[\mathrm{M}+\mathrm{H}]^{+}$Calcd for $\mathrm{C}_{16} \mathrm{H}_{16} \mathrm{ClN}_{2} \mathrm{O}_{3} 319.0844$; Found 319.0841 .

Methyl 1-(3-methylbut-2-enoyl)-3-phenyl-1H-pyrazole-4-carboxylate (4b). Prepared according to the general procedure from 1 b (143 mg, $0.5 \mathrm{mmol}) ; C C$ (EA/PE, 1:7); $126 \mathrm{mg}$ (75\% yield); white solid; $\mathrm{mp} 109-110{ }^{\circ} \mathrm{C} ; v_{\max } / \mathrm{cm}^{-1}$ (ATR) $1731,1712,1628,1138 ; \delta_{\mathrm{H}}(500 \mathrm{MHz}$; $\mathrm{CDCl}_{3}$; Me $\left.4 \mathrm{Si}\right) 8.87(\mathrm{~s}, 1 \mathrm{H}), 7.88-7.81(\mathrm{~m}, 2 \mathrm{H}), 7.51-7.43(\mathrm{~m}, 3 \mathrm{H}), 7.23-7.18(\mathrm{~m}, 1 \mathrm{H}), 3.84$ $(\mathrm{s}, 3 \mathrm{H}), 2.39(\mathrm{~d}, J=1.0 \mathrm{~Hz}, 3 \mathrm{H}), 2.13(\mathrm{~d}, J=1.0 \mathrm{~Hz}, 3 \mathrm{H}) ; \delta_{\mathrm{c}}\left(126 \mathrm{MHz} ; \mathrm{CDCl}_{3} ; \mathrm{Me}_{4} \mathrm{Si}\right)$ 165.3, 163.0, 162.4, 154.8, 134.0, 131.4, 129.3, 129.2, 128.0, 114.9, 113.4, 51.7, 28.6, 21.7; HRMS (ESI) m/z: $[\mathrm{M}+\mathrm{H}]^{+}$Calcd for $\mathrm{C}_{16} \mathrm{H}_{17} \mathrm{~N}_{2} \mathrm{O}_{3}$ 285.1234; Found 285.1233.

Methyl 1-(3-methylbut-2-enoyl)-3-(p-tolyl)-1H-pyrazole-4-carboxylate (4c). Prepared according to the general procedure from $1 \mathrm{j}$ (150 mg, $0.5 \mathrm{mmol}$ ); CC (EA/PE, 1:2); $113 \mathrm{mg}$ (76\% yield); white solid; $\mathrm{mp} 109-110^{\circ} \mathrm{C} ; v_{\max } / \mathrm{cm}^{-1}$ (ATR) $1736,1711,1630,1141,1125 ; \delta_{\mathrm{H}}(500$ $\mathrm{MHz} \mathrm{CDCl}_{3}$; $\left.\mathrm{Me}_{4} \mathrm{Si}\right) 8.86(\mathrm{~s}, 1 \mathrm{H}), 7.74(\mathrm{~d}, J=8.1 \mathrm{~Hz}, 2 \mathrm{H}), 7.30-7.26(\mathrm{~m}, 2 \mathrm{H}), 7.23-7.20(\mathrm{~m}$, $1 \mathrm{H}), 3.84(\mathrm{~s}, 3 \mathrm{H}), 2.43(\mathrm{~s}, 3 \mathrm{H}), 2.38(\mathrm{~d}, J=0.7 \mathrm{~Hz}, 3 \mathrm{H}), 2.12(\mathrm{~d}, J=0.8 \mathrm{~Hz}, 3 \mathrm{H}) ; \delta_{\mathrm{c}}(126 \mathrm{MHz}$; $\mathrm{CDCl}_{3}$; $\left.\mathrm{Me}_{4} \mathrm{Si}\right)$ 165.1, 163.1, 162.4, 154.9, 139.2, 134.0, 129.2, 128.8, 128.5, 114.9, 113.5, 51.7, 28.6, 21.7, 21.4; HRMS (ESI) m/z: [M + H] ${ }^{+}$Calcd for $\mathrm{C}_{17} \mathrm{H}_{19} \mathrm{~N}_{2} \mathrm{O}_{3}$ 299.1390; Found 299.1389.

Methyl 3-(4-cyanophenyl)-1-(3-methylbut-2-enoyl)-1H-pyrazole-4-carboxylate (4d). Prepared according to the general procedure from 1d (156 mg, $0.5 \mathrm{mmol}$ ); CC (EA/PE, 1:4); $116 \mathrm{mg}$ (75\% 
yield); white solid; $\mathrm{mp} 150-151{ }^{\circ} \mathrm{C} ; v_{\max } / \mathrm{cm}^{-1}$ (ATR) $2224,1732,1709,1626,1142 ; \delta_{\mathrm{H}}(500$ $\mathrm{MHz} ; \mathrm{CDCl}_{3}$; $\left.\mathrm{Me}_{4} \mathrm{Si}\right) 8.88(\mathrm{~s}, 1 \mathrm{H}), 8.02(\mathrm{~d}, J=8.4 \mathrm{~Hz}, 2 \mathrm{H}), 7.75(\mathrm{~d}, J=8.4 \mathrm{~Hz}, 2 \mathrm{H}), 7.20-7.12(\mathrm{~m}$, $1 \mathrm{H}), 3.85(\mathrm{~s}, 3 \mathrm{H}), 2.38(\mathrm{~d}, J=0.8 \mathrm{~Hz}, 3 \mathrm{H}), 2.14(\mathrm{~d}, J=0.9 \mathrm{~Hz}, 3 \mathrm{H}) ; \delta_{c}\left(126 \mathrm{MHz} ; \mathrm{CDCl}_{3} ; \mathrm{Me}_{4} \mathrm{Si}\right)$ 166.4, 162.7, 162.0, 152.8, 135.9, 134.4, 131.8, 130.1, 118.8, 114.9, 113.0, 112.7, 51.9, 28.7, 21.8; HRMS (ESI) m/z: [M+ H] $]^{+}$Calcd for $\mathrm{C}_{17} \mathrm{H}_{16} \mathrm{~N}_{3} \mathrm{O}_{3}$ 310.1186; Found 310.1182.

Methyl 3-(4-methoxyphenyl)-1-(3-methylbut-2-enoyl)-1H-pyrazole-4-carboxylate (4e). Prepared according to the general procedure from 1c (158 mg, $0.5 \mathrm{mmol}$ ); CC (EA/PE, 1:5); $121 \mathrm{mg}\left(77 \%\right.$ yield); white solid; $\mathrm{mp} 108-109{ }^{\circ} \mathrm{C} ; v_{\max } / \mathrm{cm}^{-1}$ (ATR) 1734, 1706, 1616, 1138; $\delta_{\mathrm{H}}$ (500 MHz; $\left.\mathrm{CDCl}_{3} ; \mathrm{Me}_{4} \mathrm{Si}\right) 8.85(\mathrm{~s}, 1 \mathrm{H}), 7.86-7.78(\mathrm{~m}, 2 \mathrm{H}), 7.22-7.19(\mathrm{~m}, 1 \mathrm{H}), 7.03-6.95(\mathrm{~m}$, $2 \mathrm{H}), 3.88(\mathrm{~s}, 3 \mathrm{H}), 3.84(\mathrm{~s}, 3 \mathrm{H}), 2.38(\mathrm{~d}, J=0.7 \mathrm{~Hz}, 3 \mathrm{H}), 2.12(\mathrm{~d}, J=0.8 \mathrm{~Hz}, 3 \mathrm{H}) ; \delta_{\mathrm{c}}(126 \mathrm{MHz}$; $\mathrm{CDCl}_{3}$; Mes $\mathrm{Si}$ ) 165.0, 163.1, 162.4, 160.5, 154.5, 134.1, 130.7, 123.8, 114.7, 113.5, 113.5, 55.3, 51.7, 28.6, 21.6; HRMS (ESI) m/z: [M + H] ${ }^{+}$Calcd for $\mathrm{C}_{17} \mathrm{H}_{19} \mathrm{~N}_{2} \mathrm{O}_{4} 315.1339$; Found 315.1334.

Methyl 3-(2,6-dichlorophenyl)-1-(3-methylbut-2-enoyl)-1H-pyrazole-4-carboxylate (4f). Prepared according to the general procedure from 1k (178 mg, $0.5 \mathrm{mmol}) ; \mathrm{CC}(\mathrm{EA} / \mathrm{PE}, 1: 7)$; $108 \mathrm{mg}$ (61\% yield); white solid; $\mathrm{mp} 140-141^{\circ} \mathrm{C} ; v_{\max } / \mathrm{cm}^{-1}$ (ATR) 1726, 1628, 1361, 1153; $\delta_{\mathrm{H}}$ (500 MHz; $\left.\mathrm{CDCl}_{3} ; \mathrm{Me}_{4} \mathrm{Si}\right) 8.91(\mathrm{~s}, 1 \mathrm{H}), 7.45-7.41(\mathrm{~m}, 2 \mathrm{H}), 7.36-7.32(\mathrm{~m}, 1 \mathrm{H}), 7.15-7.12(\mathrm{~m}$, $1 \mathrm{H}), 3.76(\mathrm{~s}, 3 \mathrm{H}), 2.39$ (d, $J=0.8 \mathrm{~Hz}, 3 \mathrm{H}), 2.10$ (d, $J=0.8 \mathrm{~Hz}, 3 \mathrm{H}) ; \delta_{c}\left(126 \mathrm{MHz} ; \mathrm{CDCl}_{3}\right.$; $\left.\mathrm{Me}_{4} \mathrm{Si}\right)$ 165.8, 162.2, 162.1, 150.5, 135.7, 132.7, 130.8, 130.6, 127.8, 116.5, 113.3, 51.7, 28.6, 21.8; HRMS (ESI) m/z: [M + H] $]^{+}$Calcd for $\mathrm{C}_{16} \mathrm{H}_{15} \mathrm{~N}_{2} \mathrm{Cl}_{2} \mathrm{O}_{3}$ 353.0454; Found 353.0449.

Methyl 1-(3-methylbut-2-enoyl)-3-(3,4,5-trimethoxyphenyl)-1H-pyrazole-4-carboxylate (4g). Prepared according to the general procedure from 11 (188 mg, $0.5 \mathrm{mmol}$ ); CC (EA/PE, 2:5); $108 \mathrm{mg}$ ( $58 \%$ yield); white solid; $\mathrm{mp} 104-105{ }^{\circ} \mathrm{C} ; v_{\max } / \mathrm{cm}^{-1}$ (ATR) $1732,1712,1617,1419$, 1398, 1117; $\delta_{\mathrm{H}}\left(500 \mathrm{MHz} ; \mathrm{CDCl}_{3} ; \mathrm{Me}_{4} \mathrm{Si}\right) 8.85$ (d, $\left.J=3.7 \mathrm{~Hz}, 1 \mathrm{H}\right), 7.18$ (dd, $\left.J=5.0,3.8 \mathrm{~Hz}, 1 \mathrm{H}\right)$, $7.16(\mathrm{~s}, 2 \mathrm{H}), 3.93(\mathrm{~s}, 6 \mathrm{H}), 3.90(\mathrm{~s}, 3 \mathrm{H}), 3.84(\mathrm{~s}, 3 \mathrm{H}), 2.37(\mathrm{~d}, J=1.0 \mathrm{~Hz}, 3 \mathrm{H}), 2.12(\mathrm{~d}, J=1.1 \mathrm{~Hz}$, $3 \mathrm{H}) ; \delta_{\mathrm{C}}\left(126 \mathrm{MHz} ; \mathrm{CDCl}_{3} ; \mathrm{Me}_{4} \mathrm{Si}\right)$ 165.5, 163.0, 162.3, 154.5, 152.9, 139.1, 134.4, 126.6, 114.9, 113.3, 106.9, 60.9, 56.3, 51.8, 28.6, 21.7; HRMS (ESI) m/z: $[\mathrm{M}+\mathrm{H}]^{+}$Calcd for $\mathrm{C}_{19} \mathrm{H}_{23} \mathrm{~N}_{2} \mathrm{O}_{6}$ 375.1551 ; Found 375.1546.

Methyl (E)-1-(3-methylbut-2-enoyl)-3-styryl-1H-pyrazole-4-carboxylate (4h) and methyl (Z)-1(3-methylbut-2-enoyl)-3-styryl-1H-pyrazole-4-carboxylate (4h'). Prepared according to the general procedure from 1i (156 mg, $0.5 \mathrm{mmol}) ; \mathbf{4 h}: \mathbf{4} \mathbf{h}^{\prime}=3.3: 1$; CC (EA/PE, 1:6); 4h: $36 \mathrm{mg}$ (23\% yield); white solid; $\mathrm{mp} 93-94^{\circ} \mathrm{C} ; v_{\max } / \mathrm{cm}^{-1}$ (ATR) 1712, 1628, 1552, 1365, 1222, 1101; $\delta_{H}$ (500 MHz; $\left.\mathrm{CDCl}_{3} ; \mathrm{Me}_{4} \mathrm{Si}\right) 8.75(\mathrm{~s}, 1 \mathrm{H}), 7.69(\mathrm{~d}, J=16.4 \mathrm{~Hz}, 1 \mathrm{H}), 7.66-7.57(\mathrm{~m}, 3 \mathrm{H}), 7.38(\mathrm{t}, J=$ $7.4 \mathrm{~Hz}, 2 \mathrm{H}), 7.31(\mathrm{t}, J=7.2 \mathrm{~Hz}, 1 \mathrm{H}), 7.20(\mathrm{~s}, 1 \mathrm{H}), 3.89(\mathrm{~s}, 3 \mathrm{H}), 2.36(\mathrm{~s}, 3 \mathrm{H}), 2.15(\mathrm{~s}, 3 \mathrm{H}) ; \delta_{\mathrm{c}}(126$ $\mathrm{MHz} ; \mathrm{CDCl}_{3}$; $\mathrm{Me}_{4} \mathrm{Si}$ ) 165.2, 163.3, 162.4, 152.2, 136.6, 134.0, 133.2, 128.7, 128.5, 127.2, 117.4, 114.9, 113.5, 51.7, 28.7, 21.7; HRMS (ESI) m/z: [M + H] Calcd for $\mathrm{C}_{18} \mathrm{H}_{19} \mathrm{~N}_{2} \mathrm{O}_{3}$ 311.1390; Found 311.1393.

Methyl 3-methyl-1-(3-methylbut-2-enoyl)-1H-pyrazole-4-carboxylate (4i). Prepared according to the general procedure from 1f (112 mg, $0.5 \mathrm{mmol}) ; \mathrm{CC}$ (EA/PE, 1:6); $72 \mathrm{mg}$ (65\% yield); white solid; $\mathrm{mp} 66-67^{\circ} \mathrm{C} ; v_{\max } / \mathrm{cm}^{-1}$ (ATR) $1732,1703,1630,1564,1273,1139,1100 ; \delta_{H}(500$ 
$\mathrm{MHz} ; \mathrm{CDCl}_{3}$; $\left.\mathrm{Me}_{4} \mathrm{Si}\right) 8.69$ (s, 1H), $7.06(\mathrm{~s}, 1 \mathrm{H}), 3.85$ (s, 3H), 2.51 (s, 3H), 2.33 (s, 3H), $2.10(\mathrm{~s}, 3 \mathrm{H})$; $\delta_{c}\left(126 \mathrm{MHz} ; \mathrm{CDCl}_{3}\right.$; $\left.\mathrm{Me}_{4} \mathrm{Si}\right)$ 164.6, 163.5, 162.3, 153.5, 132.9, 115.6, 113.5, 51.5, 28.5, 21.6, 13.8; $\mathrm{HRMS}$ (ESI) m/z: [M+ H] $]^{+}$Calcd for $\mathrm{C}_{11} \mathrm{H}_{15} \mathrm{~N}_{2} \mathrm{O}_{3}$ 223.1077; Found 223.1076.

Methyl 1-(3-methylbut-2-enoyl)-3-(naphthalen-2-yl)-1H-pyrazole-4-carboxylate (4j). Prepared according to the general procedure from $1 \mathrm{~m}$ (168 mg, $0.5 \mathrm{mmol}$ ); CC (EA/PE, 1:7); $127 \mathrm{mg}$ (76\% yield); white solid; $\mathrm{mp} 120-121^{\circ} \mathrm{C} ; v_{\max } / \mathrm{cm}^{-1}$ (ATR) 1728, 1709, 1117; $\delta_{\mathrm{H}}(500 \mathrm{MHz}$; $\mathrm{CDCl}_{3}$; $\left.\mathrm{Me}_{4} \mathrm{Si}\right) 8.89(\mathrm{~s}, 1 \mathrm{H}), 8.38(\mathrm{~s}, 1 \mathrm{H}), 7.95-7.84(\mathrm{~m}, 4 \mathrm{H}), 7.54-7.46(\mathrm{~m}, 2 \mathrm{H}), 7.23(\mathrm{~s}, 1 \mathrm{H})$, $3.83(\mathrm{~s}, 3 \mathrm{H}), 2.37$ (d, J=0.5 Hz, 3H), 2.11 (d, J=0.5 Hz, 3H); $\delta_{\mathrm{c}}\left(126 \mathrm{MHz} ; \mathrm{CDCl}_{3} ; \mathrm{Me}_{4} \mathrm{Si}\right)$ 165.4, 163.1, 162.4, 154.8, 134.2, 133.7, 133.0, 129.1, 128.8, 128.7, 127.7, 127.5, 126.8, 126.7, 126.2, 115.2, 113.4, 51.8, 28.7, 21.7; HRMS (ESI) m/z: [M + H] Calcd for $\mathrm{C}_{20} \mathrm{H}_{19} \mathrm{~N}_{2} \mathrm{O}_{3}$ 335.1390; Found 335.1385 .

1-(4-Acetyl-3-(4-methoxyphenyl)-1H-pyrazol-1-yl)-3-methylbut-2-en-1-one (4k). Prepared according to the general procedure from 1n (150 mg, $0.5 \mathrm{mmol})$; CC (EA/PE, 1:4); $75 \mathrm{mg}$ (50\% yield); white solid; $\mathrm{mp} 139-140{ }^{\circ} \mathrm{C} ; v_{\max } / \mathrm{cm}^{-1}$ (ATR) $3122,1710,1686,1614,1147 ; \delta_{H}(500$ $\left.\mathrm{MHz} ; \mathrm{CDCl}_{3} ; \mathrm{Me}_{4} \mathrm{Si}\right) 8.82(\mathrm{~s}, 1 \mathrm{H}), 7.77-7.71(\mathrm{~m}, 2 \mathrm{H}), 7.20-7.15(\mathrm{~m}, 1 \mathrm{H}), 7.00-6.93(\mathrm{~m}, 2 \mathrm{H})$, $3.86(\mathrm{~s}, 3 \mathrm{H}), 2.48(\mathrm{~s}, 3 \mathrm{H}), 2.37(\mathrm{~d}, J=0.9 \mathrm{~Hz}, 3 \mathrm{H}), 2.11(\mathrm{~d}, J=1.0 \mathrm{~Hz}, 3 \mathrm{H}) ; \delta_{\mathrm{c}}\left(126 \mathrm{MHz} ; \mathrm{CDCl}_{3}\right.$; $\left.\mathrm{Me}_{4} \mathrm{Si}\right)$ 192.4, 165.3, 162.6, 160.5, 154.1, 133.6, 130.7, 124.0, 123.1, 113.5, 113.5, 55.3, 29.4, 28.6, 21.7; HRMS (ESI) m/z: [M + H] $]^{+}$Calcd for $\mathrm{C}_{17} \mathrm{H}_{19} \mathrm{~N}_{2} \mathrm{O}_{3}$ 299.1390; Found 299.1387.

Methyl 1-(2-(((benzyloxycarbonylamino)-3-methylbut-2-enoyl)-3-phenyl-1H-pyrazole-4carboxylate (4I). Prepared according to the general procedure from 10 (218 $\mathrm{mg}, 0.5 \mathrm{mmol})$; CC (EA/PE, 1:3); $136 \mathrm{mg}$ (63\% yield); white solid; $\mathrm{mp} 106-107^{\circ} \mathrm{C} ; v_{\max } / \mathrm{cm}^{-1}$ (ATR) 3296, 1718, $1692,1518,1269 ; \delta_{H}\left(500 \mathrm{MHz} ; \mathrm{CDCl}_{3} ; \mathrm{Me}_{4} \mathrm{Si}\right) 8.80(\mathrm{~s}, 1 \mathrm{H}), 7.70(\mathrm{~d}, J=6.8 \mathrm{~Hz}, 2 \mathrm{H}), 7.47-7.34$ (m, 3H), $7.29-7.17(\mathrm{~m}, 5 \mathrm{H}), 6.62(\mathrm{~s}, 1 \mathrm{H}), 5.03(\mathrm{~s}, 2 \mathrm{H}), 3.82(\mathrm{~s}, 3 \mathrm{H}), 2.04(\mathrm{~s}, 3 \mathrm{H}), 1.94(\mathrm{~s}, 3 \mathrm{H}) ; \delta_{\mathrm{c}}$ (126 MHz; CDCl 3 ; $\mathrm{Me}_{4} \mathrm{Si}$ ) 162.66, 162.32, 155.33, 154.49, 143.71, 135.71, 135.35, 130.92, 129.36, 128.51, 128.30, 128.24, 127.97, 122.41, 114.75, 77.24, 67.46, 51.76, 20.99, 20.90; HRMS (ESI) m/z: [M - Cbz + H] ${ }^{+}$Calcd for $\mathrm{C}_{11} \mathrm{H}_{11} \mathrm{~N}_{2} \mathrm{O}_{2}$ 203.0815; Found 203.0816.

Methyl 1-(3-methylbut-2-enoyl)-3-(1-tosyl-1H-indol-3-yl)-1H-pyrazole-4-carboxylate (4m). Prepared according to the general procedure from 1p (240 mg, $0.5 \mathrm{mmol}$ ); CC (EA/PE, 1:3); $122 \mathrm{mg}$ (51\% yield); white solid; $\mathrm{mp} 199-200{ }^{\circ} \mathrm{C} ; v_{\max } / \mathrm{cm}^{-1}$ (ATR) $1715,1623,1139 ; \delta_{H}(500$ $\mathrm{MHz} ; \mathrm{CDCl}_{3}$; Me $\left.4 \mathrm{Si}\right) 8.87(\mathrm{~s}, 1 \mathrm{H}), 8.85(\mathrm{~s}, 1 \mathrm{H}), 8.29(\mathrm{~d}, J=7.1 \mathrm{~Hz}, 1 \mathrm{H}), 8.05(\mathrm{~d}, J=7.6 \mathrm{~Hz}, 1 \mathrm{H})$, $7.86(\mathrm{~d}, J=8.4 \mathrm{~Hz}, 2 \mathrm{H}), 7.35$ (dqd, $J=14.7,7.3,1.2 \mathrm{~Hz}, 2 \mathrm{H}), 7.22(\mathrm{~d}, J=8.2 \mathrm{~Hz}, 2 \mathrm{H}), 7.20-7.18$ (m, 1H), $3.91(\mathrm{~s}, 3 \mathrm{H}), 2.37$ (d, J = 0.9 Hz, 3H), $2.32(\mathrm{~s}, 3 \mathrm{H}), 2.14(\mathrm{~d}, J=1.0 \mathrm{~Hz}, 3 \mathrm{H}) ; \delta_{\mathrm{c}}(126 \mathrm{MHz}$; $\mathrm{CDCl}_{3}$; $\mathrm{Me}_{4} \mathrm{Si}$ ) 165.4, 163.0, 162.2, 148.0, 145.1, 135.1, 134.8, 134.0, 129.9 (2C), 129.1, 127.1, 125.0, 123.8, 122.6, 115.0, 113.4, 113.3, 112.8, 51.9, 28.8, 21.7, 21.6; HRMS (ESI) m/z: $[\mathrm{M}+\mathrm{H}]^{+}$ Calcd for $\mathrm{C}_{25} \mathrm{H}_{24} \mathrm{~N}_{3} \mathrm{O}_{5} \mathrm{~S}$ 478.1431; Found 478.1420.

tert-Butyl (S)-(1-(3-(4-chlorophenyl)-1-(3-methylbut-2-enoyl)-1H-pyrazol-4-yl)-1-oxopropan2-yl)carbamate (4n). Prepared according to the general procedure from 1q $(217 \mathrm{mg}, 0.5$ $\mathrm{mmol}$ ); CC (EA/PE, 1:3); $182 \mathrm{mg}$ (84\% yield); white solid; $\mathrm{mp} 134-135^{\circ} \mathrm{C} ;[\alpha]_{589}^{25}-31$ (c 0.25 , $\mathrm{MeOH}$ ); $v_{\max } / \mathrm{cm}^{-1}$ (ATR) 3327, 1714, 1688, 1666, 1631, 1139; $\delta_{\mathrm{H}}\left(500 \mathrm{MHz} ; \mathrm{CDCl}_{3} ; \mathrm{Me}_{4} \mathrm{Si}\right) 8.95$ 
(s, 1H), $7.75-7.69(\mathrm{~m}, 2 \mathrm{H}), 7.41(\mathrm{~d}, J=8.5 \mathrm{~Hz}, 2 \mathrm{H}), 7.15(\mathrm{~s}, 1 \mathrm{H}), 5.33(\mathrm{~d}, J=7.5 \mathrm{~Hz}, 1 \mathrm{H}), 4.93$ (p, J = 7.1 Hz, 1H), $2.37(\mathrm{~s}, 3 \mathrm{H}), 2.12(\mathrm{~s}, 3 \mathrm{H}), 1.44(\mathrm{~s}, 10 \mathrm{H}), 1.41(\mathrm{~d}, J=7.1 \mathrm{~Hz}, 3 \mathrm{H}) ; \delta_{c}(126 \mathrm{MHz}$; $\mathrm{CDCl}_{3}$; Me $\left.4 \mathrm{Si}\right)$ 194.4, 166.2, 162.1, 155.2, 154.0, 135.5, 133.3, 130.7, 129.9, 128.3, 119.6, 113.1, 80.0, 53.5, 28.7, 28.3, 21.8, 19.4; HRMS (ESI) m/z: [M - Boc] $]^{+}$Calcd for $\mathrm{C}_{17} \mathrm{H}_{19} \mathrm{ClN}_{3} \mathrm{O}_{2} 332.1160$; Found 332.1153.

Methyl 3-(1-methyl-1H-pyrrol-2-yl)-1-(3-methylbut-2-enoyl)-1H-pyrazole-4-carboxylate (40) and diethyl 2-(5-(4-(methoxycarbonyl)-1-(3-methylbut-2-enoyl)-1H-pyrazol-3-yl)-1-methyl$1 \mathrm{H}$-pyrrol-2-yl)malonate (4o'). Prepared according to the general procedure from $\mathbf{1 r}(145 \mathrm{mg}$, $0.5 \mathrm{mmol})$, diethyl bromomalonate $(370 \mu \mathrm{L}, 92 \%$ purity, 4.0 equiv) and 2,6-lutidine (174 $\mu \mathrm{L}$, 3.0 equiv); products were separated by CC (EA/PE, 2:7); 40; $22 \mathrm{mg}$ (15\% yield); white solid; $\mathrm{mp}$ 98-99 ${ }^{\circ} \mathrm{C} ; v_{\max } / \mathrm{cm}^{-1}(\mathrm{ATR}) 1711,1624,1123 ; \delta_{\mathrm{H}}\left(500 \mathrm{MHz} ; \mathrm{CDCl}_{3} ; \mathrm{Me}_{4} \mathrm{Si}\right) 8.82(\mathrm{~s}, 1 \mathrm{H}), 7.12$ (s, $1 \mathrm{H}), 6.79(\mathrm{~d}, J=2.0 \mathrm{~Hz}, 1 \mathrm{H}), 6.76(\mathrm{~s}, 1 \mathrm{H}), 6.25-6.16(\mathrm{~m}, 1 \mathrm{H}), 3.83(\mathrm{~s}, 3 \mathrm{H}), 3.78(\mathrm{~s}, 3 \mathrm{H}), 2.36(\mathrm{~s}$, $3 \mathrm{H}), 2.09$ (s, 3H); $\delta_{\mathrm{C}}\left(126 \mathrm{MHz} ; \mathrm{CDCl}_{3} ; \mathrm{Me}_{4} \mathrm{Si}\right)$ 165.0, 162.8, 162.3, 147.4, 133.5, 125.4, 123.1, 115.7, 113.9, 113.4, 107.7, 51.8, 36.1, 28.7, 21.6; HRMS (ESI) $\mathrm{m} / \mathrm{z}:[\mathrm{M}+\mathrm{H}]^{+}$Calcd for $\mathrm{C}_{15} \mathrm{H}_{18} \mathrm{~N}_{3} \mathrm{O}_{3}$ 288.1343; Found 288.1338. 40; $112 \mathrm{mg}$ (50\% yield); yellow oil; $v_{\mathrm{max}} / \mathrm{cm}^{-1}$ (ATR) 1716, 1627, 1133; $\delta_{\mathrm{H}}\left(500 \mathrm{MHz} ; \mathrm{CDCl}_{3} ; \mathrm{Me}_{4} \mathrm{Si}\right) 8.83(\mathrm{~s}, 1 \mathrm{H}), 7.16-7.07(\mathrm{~m}, 1 \mathrm{H}), 6.64$ (d, J = 3.9 $\mathrm{Hz}, 1 \mathrm{H}), 6.32(\mathrm{~d}, J=3.9 \mathrm{~Hz}, 1 \mathrm{H}), 4.79(\mathrm{~s}, 1 \mathrm{H}), 4.26$ (qdd, $J=14.3,9.0,5.4 \mathrm{~Hz}, 4 \mathrm{H}), 3.81(\mathrm{~s}, 3 \mathrm{H})$, $3.63(\mathrm{~s}, 3 \mathrm{H}), 2.36(\mathrm{~d}, J=1.0 \mathrm{~Hz}, 3 \mathrm{H}), 2.09(\mathrm{~d}, J=1.1 \mathrm{~Hz}, 3 \mathrm{H}), 1.31(\mathrm{t}, J=7.1 \mathrm{~Hz}, 6 \mathrm{H}) ; \delta_{\mathrm{c}}(126 \mathrm{MHz}$; $\mathrm{CDCl}_{3}$; Me $\left.4 \mathrm{Si}\right)$ 167.3, 165.4, 162.6, 162.2, 147.3, 133.4, 126.3, 124.8, 116.2, 113.3, 112.6, 109.6, 62.0, 51.8, 51.1, 32.8, 28.7, 21.7, 14.1; HRMS (ESI) $\mathrm{m} / \mathrm{z}$ : $[\mathrm{M}+\mathrm{H}]^{+}$Calcd for $\mathrm{C}_{22} \mathrm{H}_{28} \mathrm{~N}_{3} \mathrm{O}_{7}$ 446.1922; Found 446.1911.

\section{General procedure for the synthesis of pyrazoles 5}

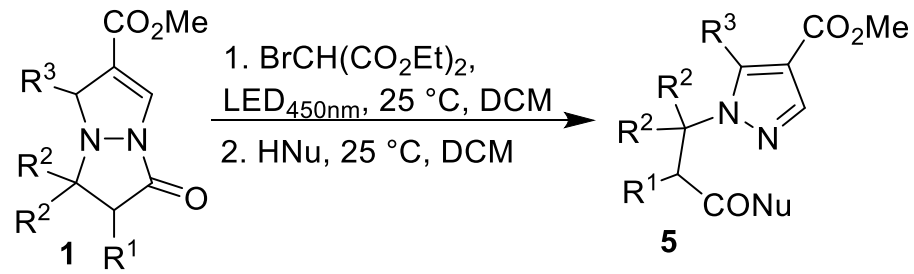

Scheme SI4: Synthesis of compounds 5.

A dried $8 \mathrm{~mL}$ vial was charged with $1(0.5 \mathrm{mmol})$ and dry DCM $(2.5 \mathrm{~mL})$ as the solvent unless noted otherwise. The vial was sealed off with a screw cap with a septum. The resulting solution was degassed via needle by three freeze-pump-thaw cycles. Diethyl bromomalonate $(185 \mu \mathrm{L}$, $92 \%$ purity, 2.0 equiv) was then added. The solution was irradiated with a blue LED $_{450} \mathrm{~nm}$ under nitrogen atmosphere for $18 \mathrm{~h}$ at $25^{\circ} \mathrm{C}$. If necessary, a solution of a nucleophile (2.0 equiv) in dry DCM $(1.0 \mathrm{~mL})$ was added and the reaction mixture stirred for an additional $1 \mathrm{~h}$ at $25^{\circ} \mathrm{C}$ without irradiation. Products $\mathbf{5}$ were isolated by CC and dried under vacuum unless noted otherwise. 


\section{Characterization data of novel compounds 5}

3-(5-(4-Chlorophenyl)-4-(methoxycarbonyl)-1H-pyrazol-1-yl)-3-methylbutanoic acid (5a). Prepared according to the general procedure from 1a $(161 \mathrm{mg}, 0.5 \mathrm{mmol})$. Acetone $(2.5 \mathrm{~mL})$ with water ( $90 \mu \mathrm{L}, 10$ equiv) is used as the solvent. the reaction mixture was evaporated and product 5 a was purified by extraction in DCM. The crude mixture was extracted with saturated aqueous sodium bicarbonate $(20 \mathrm{~mL})$. The aqueous phase was acidified with sodium hydrogen sulphate $(1 \mathrm{M}, 25 \mathrm{~mL})$ and extracted with DCM $(2 \times 20 \mathrm{~mL})$. The organic phase was dried over anhydrous sodium sulphate, filtrated and the solvent evaporated in vacuo. $140 \mathrm{mg}$ ( $83 \%$ yield); white solid; $\mathrm{mp} 146-147^{\circ} \mathrm{C} ; v_{\max } / \mathrm{cm}^{-1}$ (ATR) 1704, 1224; $\delta_{\mathrm{H}}\left(500 \mathrm{MHz} ; \mathrm{CDCl}_{3} ; \mathrm{Me}_{4} \mathrm{Si}\right) 7.95$ (s, $1 \mathrm{H}), 7.43(\mathrm{~d}, J=8.0 \mathrm{~Hz}, 2 \mathrm{H}), 7.28(\mathrm{~d}, J=8.0 \mathrm{~Hz}, 2 \mathrm{H}), 3.64(\mathrm{~s}, 3 \mathrm{H}), 2.98(\mathrm{~s}, 2 \mathrm{H}), 1.48(\mathrm{~s}, 6 \mathrm{H}) ; \delta_{\mathrm{c}}$ (126 MHz; $\mathrm{CDCl}_{3}$; $\left.\mathrm{Me}_{4} \mathrm{Si}\right)$ 174.2, 163.1, 145.0, 139.4, 135.5, 131.5, 129.8, 128.4, 114.9, 63.3, 51.2, 47.5, 29.5; HRMS (ESI) m/z: [M + H] ${ }^{+}$Calcd for $\mathrm{C}_{16} \mathrm{H}_{18} \mathrm{CIN}_{2} \mathrm{O}_{4} 337.0950$; Found 337.0948.

Methyl 5-(4-chlorophenyl)-1-(4-methoxy-2-methyl-4-oxobutan-2-yl)-1H-pyrazole-4carboxylate (5b). Prepared according to the general procedure from 1a (161 mg, $0.5 \mathrm{mmol})$. Anhydrous $\mathrm{MeOH}$ (2.5 mL) is used as solvent. CC (EA/PE, 1:4); $165 \mathrm{mg}$ (94\% yield); white solid; $\mathrm{mp} 82-84{ }^{\circ} \mathrm{C} ; v_{\max } / \mathrm{cm}^{-1}$ (ATR) 1731, 1708, 1219; $\delta_{\mathrm{H}}\left(500 \mathrm{MHz} ; \mathrm{CDCl}_{3} ; \mathrm{Me}_{4} \mathrm{Si}\right) 7.91(\mathrm{~s}, 1 \mathrm{H}), 7.45$ - $7.40(\mathrm{~m}, 2 \mathrm{H}), 7.33-7.29(\mathrm{~m}, 2 \mathrm{H}), 3.63(\mathrm{~s}, 3 \mathrm{H}), 3.60(\mathrm{~s}, 3 \mathrm{H}), 2.87(\mathrm{~s}, 2 \mathrm{H}), 1.49(\mathrm{~s}, 6 \mathrm{H}) ; \delta_{\mathrm{c}}(126$ $\mathrm{MHz} ; \mathrm{CDCl}_{3}$; $\left.\mathrm{Me}_{4} \mathrm{Si}\right)$ 170.3, 163.3, 144.8, 139.4, 135.3, 131.5, 130.3, 128.3, 114.6, 63.1, 51.6, 51.0, 46.7, 29.5; HRMS (ESI) m/z: [M+ H] ${ }^{+}$Calcd for $\mathrm{C}_{17} \mathrm{H}_{20} \mathrm{ClN}_{2} \mathrm{O}_{4}$ 351.1106; Found 351.1106.

Methyl 5-(4-chlorophenyl)-1-(2-methyl-4-oxo-4-(p-tolyloxy)butan-2-yl)-1H-pyrazole-4carboxylate (5c). Prepared according to the general procedure from 1a (161 mg, $0.5 \mathrm{mmol}$ ). p-Cresol (108 mg, 2.0 equiv) is used as the nucleophile. CC (EA/PE, 1:3); $162 \mathrm{mg}$ (76\% yield); white solid; $\mathrm{mp} 102-103{ }^{\circ} \mathrm{C} ; v_{\max } / \mathrm{cm}^{-1}$ (ATR) $1765,1704,1126 ; \delta_{\mathrm{H}}\left(500 \mathrm{MHz} ; \mathrm{CDCl}_{3} ; \mathrm{Me}_{4} \mathrm{Si}\right)$ $7.95(\mathrm{~s}, 1 \mathrm{H}), 7.41$ (d, J = 8.5 Hz, 2H), $7.31(\mathrm{~d}, J=8.5 \mathrm{~Hz}, 2 \mathrm{H}), 7.14(\mathrm{~d}, J=8.2 \mathrm{~Hz}, 2 \mathrm{H}), 6.86(\mathrm{~d}, J=$ $8.4 \mathrm{~Hz}, 2 \mathrm{H}), 3.62(\mathrm{~s}, 3 \mathrm{H}), 3.12(\mathrm{~s}, 2 \mathrm{H}), 2.32(\mathrm{~s}, 3 \mathrm{H}), 1.56(\mathrm{~s}, 6 \mathrm{H}) ; \delta_{c}\left(126 \mathrm{MHz} ; \mathrm{CDCl}_{3}\right.$; $\left.\mathrm{Me}_{4} \mathrm{Si}\right)$ 168.7, 163.2, 148.1, 144.9, 139.4, 135.6, 135.3, 131.6, 130.2, 129.9, 128.3, 121.1, 114.7, 63.2, 51.1, 47.0, 29.6, 20.9; HRMS (ESI) $\mathrm{m} / \mathrm{z}$ : $[\mathrm{M}+\mathrm{H}]^{+}$Calcd for $\mathrm{C}_{23} \mathrm{H}_{24} \mathrm{ClN}_{2} \mathrm{O}_{4}$ 427.1419; Found 427.1414 .

Methyl 5-(4-chlorophenyl)-1-(2-methyl-4-oxo-4-(propylamino)butan-2-yl)-1H-pyrazole-4carboxylate (5d) Prepared according to the general procedure from 1a (161 mg, $0.5 \mathrm{mmol}$ ). $n$-Propylamine ( $83 \mu \mathrm{L}, 2.0$ equiv) is used as the nucleophile. CC (EA/PE, 1:1); $121 \mathrm{mg}(64 \%$ yield); white solid; $\mathrm{mp} 93-95^{\circ} \mathrm{C} ; v_{\max } / \mathrm{cm}^{-1}$ (ATR) 3304, 2972, 1720, 1642, 1231; $\delta_{\mathrm{H}}(500 \mathrm{MHz}$; $\mathrm{CDCl}_{3}$; $\left.\mathrm{Me}_{4} \mathrm{Si}\right) 7.98(\mathrm{~s}, 1 \mathrm{H}), 7.43-7.37(\mathrm{~m}, 2 \mathrm{H}), 7.25-7.19(\mathrm{~m}, 2 \mathrm{H}), 5.98(\mathrm{~s}, 1 \mathrm{H}), 3.62(\mathrm{~s}, 3 \mathrm{H})$, $3.09(\mathrm{dd}, J=13.1,6.9 \mathrm{~Hz}, 2 \mathrm{H}), 2.86(\mathrm{~s}, 2 \mathrm{H}), 1.42(\mathrm{~s}, 6 \mathrm{H}), 1.41-1.33(\mathrm{~m}, 2 \mathrm{H}), 0.80(\mathrm{t}, J=7.4 \mathrm{~Hz}$, $3 \mathrm{H}) ; \delta_{\mathrm{c}}\left(126 \mathrm{MHz} ; \mathrm{CDCl}_{3}\right.$; Me $\left.4 \mathrm{Si}\right) 169.3,163.0,145.5,139.8,135.4,131.4,130.0,128.3,114.8$, 64.6, 51.1, 49.6, 41.0, 29.7, 22.7, 11.3; HRMS (ESI) $\mathrm{m} / \mathrm{z}$ : $[\mathrm{M}+\mathrm{H}]^{+}$Calcd for $\mathrm{C}_{19} \mathrm{H}_{25} \mathrm{ClN}_{3} \mathrm{O}_{3}$ 378.1579; Found 378.1572.

Methyl 5-(4-chlorophenyl)-1-(2-methyl-4-oxo-4-(p-tolylamino)butan-2-yl)-1H-pyrazole-4carboxylate (5e). Prepared according to the general procedure from 1a (161 mg, $0.5 \mathrm{mmol}$ ). p-Toluidine (108 mg, 2.0 equiv) is used as the nucleophile. CC (EA/PE, 1:3); $134 \mathrm{mg}$ (63\% yield); white solid; $\mathrm{mp} 94-95^{\circ} \mathrm{C} ; v_{\max } / \mathrm{cm}^{-1}$ (ATR) 2984, 1720, 1651, 1220; $\delta_{\mathrm{H}}\left(500 \mathrm{MHz} ; \mathrm{CDCl}_{3} ; \mathrm{Me}_{4} \mathrm{Si}\right)$ $8.09(\mathrm{~s}, 1 \mathrm{H}), 7.93(\mathrm{~s}, 1 \mathrm{H}), 7.38(\mathrm{~d}, J=8.0 \mathrm{~Hz}, 2 \mathrm{H}), 7.24(\mathrm{~d}, J=8.0 \mathrm{~Hz}, 2 \mathrm{H}), 7.16(\mathrm{~d}, J=8.0 \mathrm{~Hz}, 2 \mathrm{H})$, 
$7.08(\mathrm{~d}, J=8.0 \mathrm{~Hz}, 2 \mathrm{H}), 3.63(\mathrm{~s}, 3 \mathrm{H}), 3.07(\mathrm{~s}, 2 \mathrm{H}), 2.29(\mathrm{~s}, 3 \mathrm{H}), 1.45(\mathrm{~s}, 6 \mathrm{H}) ; \delta_{\mathrm{c}}\left(126 \mathrm{MHz} ; \mathrm{CDCl}_{3}\right.$; $\left.\mathrm{Me}_{4} \mathrm{Si}\right)$ 167.5, 162.9, 145.8, 139.9, 135.5, 135.3, 133.8, 131.4, 129.6, 129.5, 128.4, 119.5, 115.2, 65.0, 51.2, 50.9, 29.7, 20.9; HRMS (ESI) m/z: [M+ H] ${ }^{+}$Calcd for $\mathrm{C}_{23} \mathrm{H}_{25} \mathrm{ClN}_{3} \mathrm{O}_{3}$ 426.1579; Found 426.1572 .

Methyl (S)-5-(4-chlorophenyl)-1-(4-((1-methoxy-1-oxopropan-2-yl)amino)-2-methyl-4oxobutan-2-yl)-1H-pyrazole-4-carboxylate (5f). Prepared according to the general procedure from 1a (161 mg, $0.5 \mathrm{mmol}$ ). The solution of the nucleophile was prepared by stirring L-alanine methyl ester hydrochloride (140 mg, 2.0 equiv) with DBU (150 $\mu \mathrm{L}, 2.0$ equiv) in dry DCM (1 mL) overnight. CC (EA/PE, 1:1); $126 \mathrm{mg}$ (60\% yield); colorless oil; $[\alpha]_{589}^{25}-26$ (c 0.17, MeOH); $v_{\text {max }} / \mathrm{cm}^{-1}$ (ATR) 3335, 2951, 1718, 1656, 1210, 1158, 729; $\delta_{\mathrm{H}}\left(500 \mathrm{MHz} ; \mathrm{CDCl}_{3} ; \mathrm{Me}_{4} \mathrm{Si}\right) 7.99$ (s, $1 \mathrm{H}), 7.41(\mathrm{~d}, J=8.7 \mathrm{~Hz}, 2 \mathrm{H}), 7.32-7.28(\mathrm{~m}, 1 \mathrm{H}), 7.26-7.22(\mathrm{~m}, 1 \mathrm{H}), 6.52(\mathrm{~d}, J=7.4 \mathrm{~Hz}, 1 \mathrm{H})$, $4.47(\mathrm{p}, J=7.2 \mathrm{~Hz}, 1 \mathrm{H}), 3.70(\mathrm{~s}, 3 \mathrm{H}), 3.63(\mathrm{~s}, 3 \mathrm{H}), 3.00(\mathrm{~d}, J=13.7 \mathrm{~Hz}, 1 \mathrm{H}), 2.85(\mathrm{~d}, J=13.7 \mathrm{~Hz}$, $1 \mathrm{H}), 1.43(\mathrm{~s}, 6 \mathrm{H}), 1.28$ (d, J = 7.2 Hz, 3H); $\delta_{\mathrm{c}}(126 \mathrm{MHz}$; CDCl ; Me $4 \mathrm{Si})$ 173.0, 168.9, 163.1, 145.4, 139.7, 135.3, 131.6, 131.4, 130.1, 128.3, 128.3, 114.8, 64.4, 52.3, 51.09, 49.1, 47.8, 29.8, 29.4, 18.1; HRMS (ESI) m/z: [M + H] Calcd for $\mathrm{C}_{20} \mathrm{H}_{25} \mathrm{CIN}_{3} \mathrm{O}_{5}$ 422.1477; Found 422.1473.

3-(4-(Methoxycarbonyl)-5-phenyl-1H-pyrazol-1-yl)propanoic acid (5g). Prepared according to the general procedure from $1 \mathrm{~g}(129 \mathrm{mg}, 0.5 \mathrm{mmol})$. Acetone $(2.5 \mathrm{~mL})$ with water $(90 \mu \mathrm{L}$, 10 equiv) was used as the solvent. Reaction mixture was evaporated and product $\mathbf{5 g}$ was purified by extraction whit DCM $(20 \mathrm{~mL})$ and washed with saturated aqueous sodium bicarbonate $(20 \mathrm{~mL})$. The aqueous phase was acidified with sodium hydrogen sulphate (1M, $25 \mathrm{~mL})$ and extracted with DCM $(2 \times 20 \mathrm{~mL})$. The organic phase was dried over anhydrous sodium sulphate, filtrated and the solvent evaporated in vacuo. $99 \mathrm{mg}$ ( $72 \%$ yield); white solid; $\mathrm{mp} 111-112{ }^{\circ} \mathrm{C} ; v_{\max } / \mathrm{cm}^{-1}$ (ATR) 2950, 1718, 1189; $\delta_{\mathrm{H}}\left(500 \mathrm{MHz} ; \mathrm{CDCl}_{3} ; \mathrm{Me}_{4} \mathrm{Si}\right) 9.05$ (bs, $\left.1 \mathrm{H}\right)$, $8.01(\mathrm{~s}, 1 \mathrm{H}), 7.51-7.45(\mathrm{~m}, 3 \mathrm{H}), 7.38-7.33(\mathrm{~m}, 2 \mathrm{H}), 4.24(\mathrm{t}, J=6.8 \mathrm{~Hz}, 2 \mathrm{H}), 3.68(\mathrm{~s}, 3 \mathrm{H}), 2.88$ $(\mathrm{t}, J=6.8 \mathrm{~Hz}, 2 \mathrm{H}) ; \delta_{\mathrm{c}}\left(126 \mathrm{MHz} ; \mathrm{CDCl}_{3} ; \mathrm{Me}_{4} \mathrm{Si}\right) 175.3,163.3,146.5,141.5,129.8,129.6,128.5$, 128.4, 112.6, 51.2, 44.6, 34.1; HRMS (ESI) m/z: [M + H] $]^{+}$Calcd for $\mathrm{C}_{14} \mathrm{H}_{15} \mathrm{~N}_{2} \mathrm{O}_{4}$ 275.1026; Found 275.1023.

Methyl 1-(3-methoxy-3-oxopropyl)-5-phenyl-1H-pyrazole-4-carboxylate (5h). Prepared according to the general procedure from $1 \mathrm{~g}(129 \mathrm{mg}, 0.5 \mathrm{mmol})$. Anhydrous $\mathrm{MeOH}(2.5 \mathrm{~mL})$ was used as the solvent. CC (EA/PE, 1:2); $133 \mathrm{mg}$ (92\% yield); colorless oil; $v_{\max } / \mathrm{cm}^{-1}$ (ATR) 2952, 1712, 1203; $\delta_{\mathrm{H}}\left(500 \mathrm{MHz} ; \mathrm{CDCl}_{3} ; \mathrm{Me}_{4} \mathrm{Si}\right) 7.99(\mathrm{~s}, J=7.2 \mathrm{~Hz}, 1 \mathrm{H}), 7.53-7.44(\mathrm{~m}, 3 \mathrm{H}), 7.42$ $-7.35(\mathrm{~m}, 2 \mathrm{H}), 4.24(\mathrm{t}, J=7.1 \mathrm{~Hz}, 2 \mathrm{H}), 3.67(\mathrm{~s}, 3 \mathrm{H}), 3.63(\mathrm{~s}, 3 \mathrm{H}), 2.87(\mathrm{t}, J=7.1 \mathrm{~Hz}, 2 \mathrm{H}) ; \delta_{\mathrm{c}}(126$ $\mathrm{MHz} ; \mathrm{CDCl}_{3}$; Me $\left.4 \mathrm{Si}\right)$ 170.9, 163.3, 146.3, 141.5, 129.9, 129.5, 128.7, 128.4, 112.5, 51.9, 51.1, 44.8, 33.9; HRMS (ESI) m/z: [M + H] $]^{+}$Calcd for $\mathrm{C}_{15} \mathrm{H}_{17} \mathrm{~N}_{2} \mathrm{O}_{4}$ 289.1183; Found 289.1174.

(RS)-2-(((Benzyloxy)carbonyl)amino)-3-(4-(methoxycarbonyl)-5-phenyl-1H-pyrazol-1-yl)-3methylbutanoic acid (5i). Prepared according to the general procedure from $101218 \mathrm{mg}$, $0.5 \mathrm{mmol})$. Acetone $(2.5 \mathrm{~mL}$ ) with water $(90 \mu \mathrm{L}, 10$ equiv) was used as the solvent. Reaction mixture was evaporated and product $5 \mathbf{i}$ purified by extraction with DCM $(20 \mathrm{~mL})$. The crude mixture was extracted with saturated aqueous sodium bicarbonate $(20 \mathrm{~mL})$. The aqueous phase was acidified with sodium hydrogen sulphate $(1 \mathrm{M}, 25 \mathrm{~mL})$ and extracted with DCM $(2 \mathrm{x}$ $20 \mathrm{~mL}$ ). The organic phase was dried over anhydrous sodium sulphate, filtrated and the solvent evaporated in vacuo. $221 \mathrm{mg}$ ( $98 \%$ yield); white solid; $\mathrm{mp} 138-139^{\circ} \mathrm{C} ; v_{\max } / \mathrm{cm}^{-1}$ (ATR) 3401, 2943, 1721, 1514, 697; $\delta_{\mathrm{H}}\left(500 \mathrm{MHz} ; \mathrm{CDCl}_{3} ; \mathrm{Me}_{4} \mathrm{Si}\right) 10.38$ (bs, $1 \mathrm{H}$ ), 7.87 (d, J = $4.0 \mathrm{~Hz}$, 
$1 \mathrm{H}), 7.51-7.18(\mathrm{~m}, 10 \mathrm{H}), 6.30(\mathrm{~d}, J=9.8 \mathrm{~Hz}, 1 \mathrm{H}), 5.13(\mathrm{~d}, J=12.2 \mathrm{~Hz}, 1 \mathrm{H}), 5.08(\mathrm{~d}, J=12.2 \mathrm{~Hz}$, $1 \mathrm{H}), 4.70(\mathrm{~d}, J=9.8 \mathrm{~Hz}, 1 \mathrm{H}), 3.58(\mathrm{~s}, 3 \mathrm{H}), 1.48(\mathrm{~s}, 3 \mathrm{H}), 1.46(\mathrm{~s}, 3 \mathrm{H}) ; \delta_{\mathrm{c}}\left(126 \mathrm{MHz} ; \mathrm{CDCl}_{3} ; \mathrm{Me}_{4} \mathrm{Si}\right)$ $173.8,163.2$, 156.6, 146.6, 139.7, 136.0, 130.9, 130.3, 129.8, 129.3, 128.5, 128.2, 128.1, 128.0, 128.0, 114.7, 67.4, 67.0, 62.8, 51.1, 27.8, 27.3; HRMS (ESI) m/z: [M + H] Calcd for $\mathrm{C}_{24} \mathrm{H}_{26} \mathrm{~N}_{3} \mathrm{O}_{6}$ 452.1816; Found 452.1818.

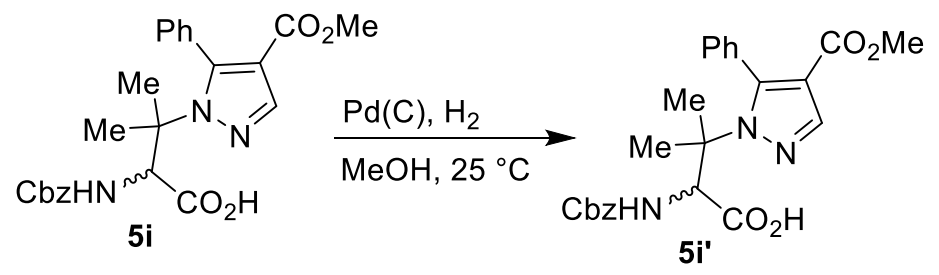

Scheme SI5: Deprotection of compound 5i.

(RS)-2-Amino-3-(4-(methoxycarbonyl)-5-phenyl-1H-pyrazol-1-yl)-3-methylbutanoic acid (5i'). $5 \mathbf{i}(180 \mathrm{mg}, 0.4 \mathrm{mmol})$ was dissolved in $\mathrm{MeOH}(10 \mathrm{~mL}$ ). Palladium on carbon (10\%, $40 \mathrm{mg}$ ) was added and the suspension was hydrogenated at 2.0 bar at $25^{\circ} \mathrm{C}$ for $6 \mathrm{~h}$. The reaction mixture was then filtered through a pad of silica and washed with $\mathrm{MeOH}(10 \mathrm{~mL})$. After evaporation of the solvent, product $5 \mathbf{i}^{\prime}$ can be further purified by $\mathrm{CC}(\mathrm{DCM} / \mathrm{MeOH}, 8: 1)$ to give a white powder. $120 \mathrm{mg}$ (95\% yield); white solid; mp $166-167^{\circ} \mathrm{C} ; v_{\max } / \mathrm{cm}^{-1}$ (ATR) 3115, 2989, 2950, $1721,1618,1547 ; \delta_{H}\left(500 \mathrm{MHz} ; \mathrm{CD}_{3} \mathrm{OD} ; \mathrm{Me}_{4} \mathrm{Si}\right) 7.98(\mathrm{~s}, 1 \mathrm{H}), 7.50-7.39(\mathrm{~m}, 4 \mathrm{H}), 7.35-7.29$ $(\mathrm{m}, 1 \mathrm{H}), 4.12$ (bs, $1 \mathrm{H}), 3.54(\mathrm{~s}, 3 \mathrm{H}), 3.34(\mathrm{~s}, 1 \mathrm{H}), 1.60(\mathrm{~s}, 3 \mathrm{H}), 1.38(\mathrm{~s}, 3 \mathrm{H}) ; \delta_{\mathrm{c}}\left(126 \mathrm{MHz} ; \mathrm{CDCl}_{3}\right.$; $\left.\mathrm{Me}_{4} \mathrm{Si}\right)$ 172.4, 165.1, 148.6, 141.7, 133.0, 132.3, 131.3, 130.5, 129.3, 116.3, 67.4, 64.9, 51.8, 50.2, 29.6, 25.5; HRMS (ESI) m/z: [M + H] ${ }^{+}$Calcd for $\mathrm{C}_{16} \mathrm{H}_{20} \mathrm{~N}_{3} \mathrm{O}_{4} 318.1448$; Found 318.1448.

Methyl (RS)-1-(3-(((benzyloxy)carbonyl)amino)-4-methoxy-2-methyl-4-oxobutan-2-yl)-5phenyl-1H-pyrazole-4-carboxylate (5j). Prepared according to the general procedure from 10 (218 mg, $0.5 \mathrm{mmol})$. Anhydrous $\mathrm{MeOH}(2.5 \mathrm{~mL})$ was used as the solvent. CC (EA/PE, 1:4); 175 $\mathrm{mg}\left(75 \%\right.$ yield); white solid; $\mathrm{mp} 132-133^{\circ} \mathrm{C} ; v_{\mathrm{max}} / \mathrm{cm}^{-1}$ (ATR) $3307,1738,1719,1151 ; \delta_{H}(500$ $\left.\mathrm{MHz} ; \mathrm{CDCl}_{3} ; \mathrm{Me}_{4} \mathrm{Si}\right) 7.86(\mathrm{~s}, 1 \mathrm{H}), 7.50-7.27(\mathrm{~m}, 1 \mathrm{HH}), 6.19(\mathrm{~d}, J=9.8 \mathrm{~Hz}, 1 \mathrm{H}), 5.11(\mathrm{~s}, 2 \mathrm{H}), 4.65$ $(\mathrm{d}, J=9.9 \mathrm{~Hz}, 1 \mathrm{H}), 3.63(\mathrm{~s}, 3 \mathrm{H}), 3.60(\mathrm{~s}, 3 \mathrm{H}), 1.50(\mathrm{~s}, 3 \mathrm{H}), 1.45(\mathrm{~s}, 3 \mathrm{H}) ; \delta_{c}\left(126 \mathrm{MHz} ; \mathrm{CDCl}_{3} ; \mathrm{Me}_{4} \mathrm{Si}\right)$ $170.1,163.2$, 156.3, 146.7, 139.5, 136.1, 131.2, 130.2, 129.9, 129.3, 128.6, 128.3, 128.1, 128.0, 127.97, 114.6, 67.2, 66.8, 62.7, 52.6, 51.0, 27.4, 27.2; HRMS (ESI) m/z: [M+ H] ${ }^{+}$Calcd for $\mathrm{C}_{25} \mathrm{H}_{28} \mathrm{~N}_{3} \mathrm{O}_{6}$ 466.1973; Found 466.1965.

Methyl 5-(furan-2-yl)-1-(4-methoxy-2-methyl-4-oxobutan-2-yl)-1H-pyrazole-4-carboxylate (5k). Prepared according to the general procedure from $1 \mathrm{e}(138 \mathrm{mg}, 0.5 \mathrm{mmol})$. Anhydrous $\mathrm{MeOH}(2.5 \mathrm{~mL}$ ) was used as the solvent. CC (EA/PE, 1:4); $80 \mathrm{mg}$ (52\% yield); pale yellow oil; $v_{\max } / \mathrm{cm}^{-1}$ (ATR) 2952, 1722, 1213, 1145; $\delta_{\mathrm{H}}\left(500 \mathrm{MHz} ; \mathrm{CDCl}_{3} ; \mathrm{Me}_{4} \mathrm{Si}\right) 7.92$ (s, 1H), $7.63-7.60$ $(\mathrm{m}, 1 \mathrm{H}), 6.61-6.59(\mathrm{~m}, 1 \mathrm{H}), 6.56(\mathrm{dd}, J=3.3,1.9 \mathrm{~Hz}, 1 \mathrm{H}), 3.70(\mathrm{~s}, 3 \mathrm{H}), 3.59(\mathrm{~s}, 3 \mathrm{H}), 2.90(\mathrm{~s}, 2 \mathrm{H})$, 1.55 (s, 6H); $\delta_{c}\left(126 \mathrm{MHz} \mathrm{CDCl}_{3}\right.$; $\left.\mathrm{Me}_{4} \mathrm{Si}\right)$ 170.3, 163.0, 143.1, 141.1, 139.2, 134.8, 116.6, 114.0, 111.4, 62.8, 51.6, 51.3, 46.4, 28.1; HRMS (ESI) $\mathrm{m} / \mathrm{z}$ : $[\mathrm{M}+\mathrm{H}]^{+}$Calcd for $\mathrm{C}_{15} \mathrm{H}_{19} \mathrm{~N}_{2} \mathrm{O}_{5} 307.1288$; Found 307.1284 .

Methyl 1-(3-(4-bromobutoxy)-3-oxopropyl)-5-phenyl-1H-pyrazole-4-carboxylate (5I). Prepared according to the general procedure from $1 \mathrm{~g}(129 \mathrm{mg}, 0.5 \mathrm{mmol})$. Anhydrous THF $\left(2.5 \mathrm{~mL}\right.$ ) was used as the solvent. CC (EA/PE, 2:5); $137 \mathrm{mg}$ (67\% yield); colorless oil; $v_{\mathrm{max}} / \mathrm{cm}^{-1}$ 
(ATR) 2951, 1716, 1552; $\delta_{\mathrm{H}}\left(500 \mathrm{MHz} ; \mathrm{CDCl}_{3} ; \mathrm{Me}_{4} \mathrm{Si}\right) 7.99(\mathrm{~s}, 1 \mathrm{H}), 7.53-7.45(\mathrm{~m}, 3 \mathrm{H}), 7.42-$ $7.35(\mathrm{~m}, 2 \mathrm{H}), 4.24(\mathrm{t}, J=7.0 \mathrm{~Hz}, 2 \mathrm{H}), 4.07(\mathrm{t}, J=6.3 \mathrm{~Hz}, 2 \mathrm{H}), 3.67(\mathrm{~s}, 3 \mathrm{H}), 3.39(\mathrm{t}, J=6.6 \mathrm{~Hz}, 2 \mathrm{H})$, $2.86(\mathrm{t}, J=7.0 \mathrm{~Hz}, 2 \mathrm{H}), 1.91-1.82(\mathrm{~m}, 2 \mathrm{H}), 1.78-1.69(\mathrm{~m}, 2 \mathrm{H}) ; \delta_{\mathrm{c}}\left(126 \mathrm{MHz} ; \mathrm{CDCl}_{3} ; \mathrm{Me}_{4} \mathrm{Si}\right)$ 170.5, 163.3, 146.2, 141.4, 129.9, 129.5, 128.6, 128.4, 112.5, 63.8, 51.1, 44.9, 34.0, 33.0, 29.1, 27.1; HRMS (ESI) m/z: [M + H] ${ }^{+}$Calcd for $\mathrm{C}_{18} \mathrm{H}_{22} \mathrm{BrN}_{2} \mathrm{O}_{4} 409.0757$; Found 409.0751.

Methyl 1-(4-(4-bromobutoxy)-2-methyl-4-oxobutan-2-yl)-5-(4-chlorophenyl)-1H-pyrazole-4carboxylate $(5 \mathrm{~m})$. Prepared according to the general procedure from $1 \mathrm{a}$ (161 $\mathrm{mg}, 0.5 \mathrm{mmol})$. Anhydrous THF (2.5 mL) was used as the solvent. CC (EA/PE, 1:4); $122 \mathrm{mg}$ (52\% yield); colorless oil; $v_{\max } / \mathrm{cm}^{-1}$ (ATR) 2950, 1719, 1547; $\delta_{\mathrm{H}}\left(500 \mathrm{MHz} ; \mathrm{CDCl}_{3} ; \mathrm{Me}_{4} \mathrm{Si}\right) 7.90(\mathrm{~s}, 1 \mathrm{H}), 7.43$ (d, J = 8.5 $\mathrm{Hz}, 2 \mathrm{H}), 7.31(\mathrm{~d}, J=8.5 \mathrm{~Hz}, 2 \mathrm{H}), 4.03(\mathrm{t}, J=6.3 \mathrm{~Hz}, 2 \mathrm{H}), 3.63(\mathrm{~s}, 3 \mathrm{H}), 3.40(\mathrm{t}, J=6.6 \mathrm{~Hz}, 2 \mathrm{H}), 2.87$ $(\mathrm{s}, 2 \mathrm{H}), 1.91-1.82(\mathrm{~m}, 2 \mathrm{H}), 1.76-1.68(\mathrm{~m}, 2 \mathrm{H}), 1.49(\mathrm{~s}, 6 \mathrm{H}) ; \delta_{\mathrm{C}}\left(126 \mathrm{MHz} ; \mathrm{CDCl}_{3} ; \mathrm{Me}_{4} \mathrm{Si}\right)$ 169.8, 163.2, 144.8, 139.3, 135.3, 131.6, 130.2, 128.3, 114.7, 63.5, 63.1, 51.1, 46.9, 33.0, 29.5, 29.2, 27.2; HRMS (ESI) m/z: [M+ H] ${ }^{+}$Calcd for $\mathrm{C}_{20} \mathrm{H}_{25} \mathrm{BrClN}_{2} \mathrm{O}_{4} 471.0681$; Found 471.0672 .

\section{Gram-scale synthesis of $4 a$}

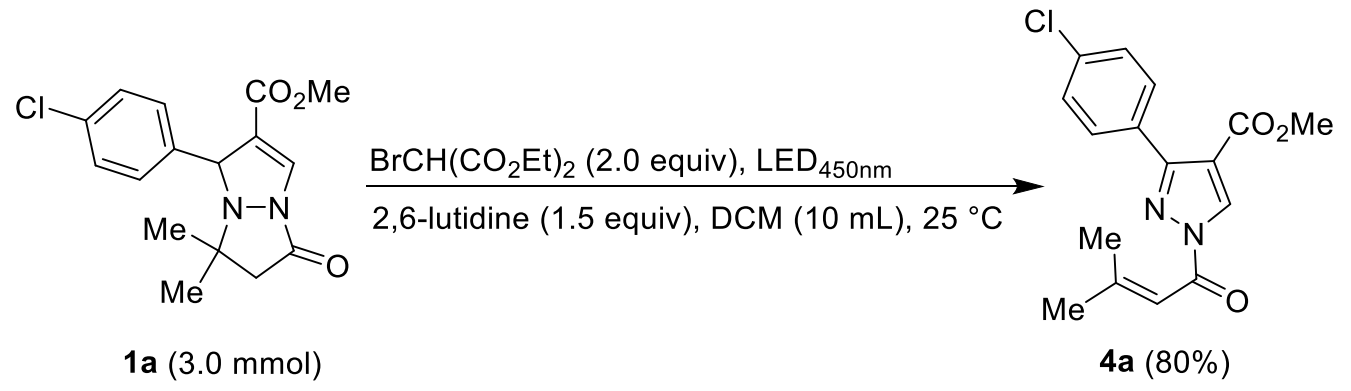

Scheme SI6: Synthesis of compound 4 a on a gram-scale.

Modified general procedure was applied on a gram-scale synthesis of 4a. A dried $25 \mathrm{~mL}$ glass tube was charged with 1a $(962 \mathrm{mg}, 3.0 \mathrm{mmol})$, diethyl bromomalonate $(1.11 \mathrm{~mL}, 92 \%$ purity, 2.0 equiv), 2,6-lutidine (522 $\mu \mathrm{L}, 1.5$ equiv) and dry DCM $(10 \mathrm{~mL})$ and sealed off with a septum. The resulting solution was degassed via needle by three freeze-pump-thaw cycles and irradiated with a blue $\mathrm{LED}_{450} \mathrm{~nm}$ under nitrogen atmosphere for 8 days at room temperature. The product 4a was purified by CC (EA/PE, 1:6) and isolated as a white solid (764 mg, 80\%). For characterization see section 8. 


\section{Mechanistic studies}

\subsection{Synthesis of deuterated analogue 2D}

Deuterated substrate 1D was prepared according to a slightly modified general procedure in order to determine the origin of the aldehyde proton in compounds 2. After irradiation, 2D was isolated in a high yield with unchanged isotopic purity.

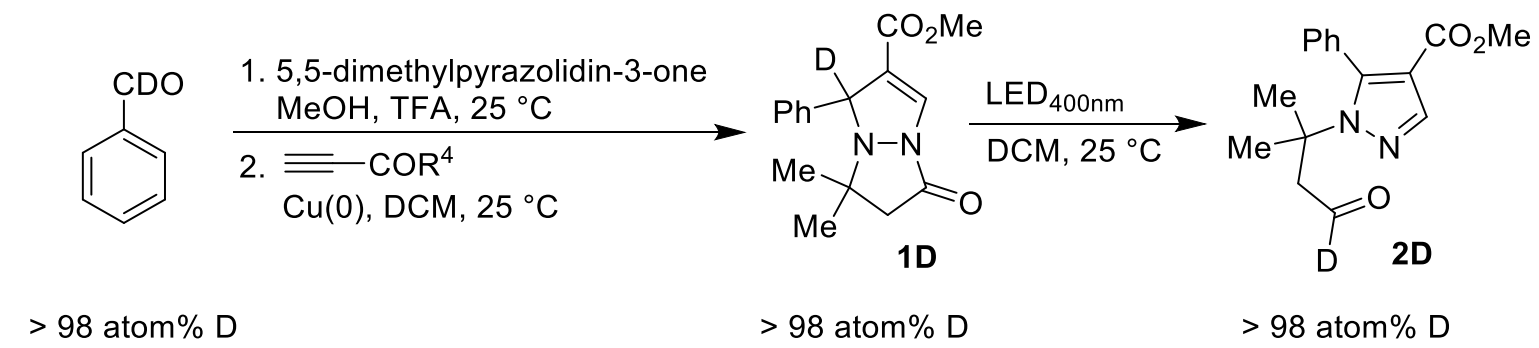

Scheme SI7: Synthesis of deuterated compounds 1D and 2D.

Methyl 7,7-dimethyl-5-oxo-1-phenyl-6,7-dihydro-1H,5H-pyrazolo[1,2-a]pyrazole-2carboxylate-1-d (1D). Prepared by stirring a solution of 5,5-dimethylpyrazolidin-3-one (342 mg, $3.0 \mathrm{mmol}$ ), benzaldehyde- $\alpha-d_{1}(337 \mathrm{mg}, 3.15 \mathrm{mmol}, 98 \mathrm{atom} \% \mathrm{D}$ ) and TFA (10 $\mu \mathrm{L}$ ) in $\mathrm{MeOH}(6 \mathrm{~mL})$ at $25{ }^{\circ} \mathrm{C}$ for $24 \mathrm{~h}$. After evaporating the volatiles, copper powder $(120 \mathrm{mg})$, $\mathrm{CH}_{2} \mathrm{Cl}_{2}(15 \mathrm{~mL})$ and methyl propiolate $(321 \mu \mathrm{L}, 3.6 \mathrm{mmol})$ were added and the mixture was stirred at $25{ }^{\circ} \mathrm{C}$ for $24 \mathrm{~h}$. The catalyst and impurities were removed by CC on silica (DCM/MeOH, 40:1) and the filtrate evaporated in vacuo to give 1D as yellow crystals. $517 \mathrm{mg}$ (60\% yield); yellow solid; $\mathrm{mp} 153-154^{\circ} \mathrm{C} ; v_{\max } / \mathrm{cm}^{-1}$ (ATR) 3074, 1787, 1735, 1688, 1595; $\delta_{\mathrm{H}}$ $\left(500 \mathrm{MHz} ; \mathrm{CDCl}_{3} ; \mathrm{Me}_{4} \mathrm{Si}\right) 7.52(\mathrm{~s}, 1 \mathrm{H}), 7.46-7.42(\mathrm{~m}, 2 \mathrm{H}), 7.38-7.32(\mathrm{~m}, 2 \mathrm{H}), 7.31-7.27(\mathrm{~m}$, $1 \mathrm{H}), 3.62(\mathrm{~s}, 3 \mathrm{H}), 2.87(\mathrm{~d}, J=15.7 \mathrm{~Hz}, 1 \mathrm{H}), 2.39(\mathrm{~d}, J=15.7 \mathrm{~Hz}, 1 \mathrm{H}), 1.24(\mathrm{~s}, 3 \mathrm{H}), 1.15(\mathrm{~s}, 3 \mathrm{H}) ; \delta \mathrm{c}$ (126 MHz; $\left.\mathrm{CDCl}_{3} ; \mathrm{Me}_{4} \mathrm{Si}\right)$ 166.6, 164.1, 141.9, 129.5, 128.4, 127.9, 127.8, 116.7, 64.5, 64.2 (JCD $=21.0 \mathrm{~Hz}$ )51.5, 49.4, 24.9, 19.0; HRMS (ESI) m/z: [M+ H] $]^{+}$Calcd for $\mathrm{C}_{16} \mathrm{H}_{18} \mathrm{DN}_{2} \mathrm{O}_{3}$ 288.1453; Found 288.1454. According to ${ }^{1} \mathrm{H}$ NMR and HRMS, isotopic purity is estimated to be $>98$ atom\% D.

Methyl 1-(2-methyl-4-oxobutan-2-yl-4-d)-5-phenyl-1H-pyrazole-4-carboxylate (2D) Prepared according to the general procedure from 1D (144 mg, $0.5 \mathrm{mmol}$ ); CC(EA/PE, 1:3); $123 \mathrm{mg}$ (86\% yield); white solid; $\mathrm{mp} 97-98^{\circ} \mathrm{C} ; v_{\max } / \mathrm{cm}^{-1}$ (ATR) 2977, 2125, 1716, 1701, 1545; $\delta_{\mathrm{H}}(500 \mathrm{MHz}$; $\mathrm{CDCl}_{3}$; $\left.\mathrm{Me}_{4} \mathrm{Si}\right) 7.91(\mathrm{~s}, 1 \mathrm{H}), 7.52-7.40(\mathrm{~m}, 3 \mathrm{H}), 7.37-7.29(\mathrm{~m}, 2 \mathrm{H}), 3.61(\mathrm{~s}, 3 \mathrm{H}), 2.91(\mathrm{~s}, 2 \mathrm{H})$, $1.47(\mathrm{~s}, 6 \mathrm{H}) ; \delta_{\mathrm{C}}\left(126 \mathrm{MHz} ; \mathrm{CDCl}_{3} ; \mathrm{Me}_{4} \mathrm{Si}\right) 199.2\left(J_{C D}=27.0 \mathrm{~Hz}\right), 163.2,145.9,139.3,131.3,130.2$, 129.2, 128.1, 114.9, 63.3, $54.9\left(J_{C D}=3.5 \mathrm{~Hz}\right), 51.0,29.5 ; \mathrm{HRMS}(\mathrm{ESI}) \mathrm{m} / \mathrm{z}$ : $[\mathrm{M}+\mathrm{H}]^{+}$Calcd for $\mathrm{C}_{16} \mathrm{H}_{18} \mathrm{DN}_{2} \mathrm{O}_{3} 288.1453$; Found 288.1453. According to ${ }^{1} \mathrm{H}$ NMR and HRMS analysis, isotopic purity was estimated to be $>98$ atom\% D.

An additional experiment including a mixture of 1D and 1d showed no intermolecular proton/deuterium exchange and both $\mathbf{2 D}$ and $\mathbf{2 d}$ were isolated as isotopically pure (> 98 atom\%) substances. Carried out according to the general procedure from 1D (14.4 mg, $0.1 \mathrm{mmol}$ ) and 1d (15.6 mg, $0.1 \mathrm{mmol}) ; \mathrm{CC}$ (EA/PE, 1:2); $12.4 \mathrm{mg}$ (86\% yield) 2D; $13.3 \mathrm{mg}$ (85\% yield) 2 d. 


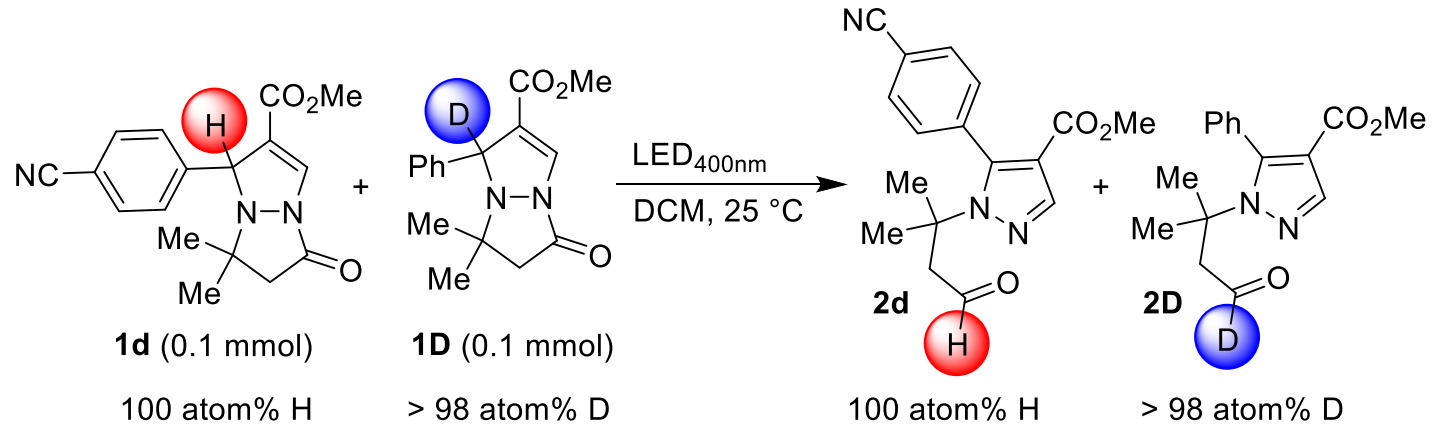

Scheme SI8: Irradiation of a mixture of compounds 1d and 1D.

\subsection{Reaction monitoring and determination of the triplet state}

Transformation from $1 \mathrm{a}$ to $\mathbf{2 a}$ was also monitored by ${ }^{1} \mathrm{H}$ NMR spectroscopy. After following the general procedure with $1 \mathrm{a}(9.6 \mathrm{mg}, 0.03 \mathrm{mmol})$ and $\mathrm{CD}_{2} \mathrm{Cl}_{2}(1.0 \mathrm{~mL})$, the reaction mixture was transferred to an NMR tube under argon and irradiated with LED $400 \mathrm{~nm}$. Progress of the reaction was monitored every hour. A parallel reaction mixture was prepared by adding transstilbene (5.4 mg, 1.0 equiv). Significant inhibition of the reaction was observed, together with the conversion of trans to cis-stilbene, which implies the energy transfer took place between the triplet excited state of $1 \mathrm{a}$ and trans-stilbene (Figure SI1). ${ }^{14}$

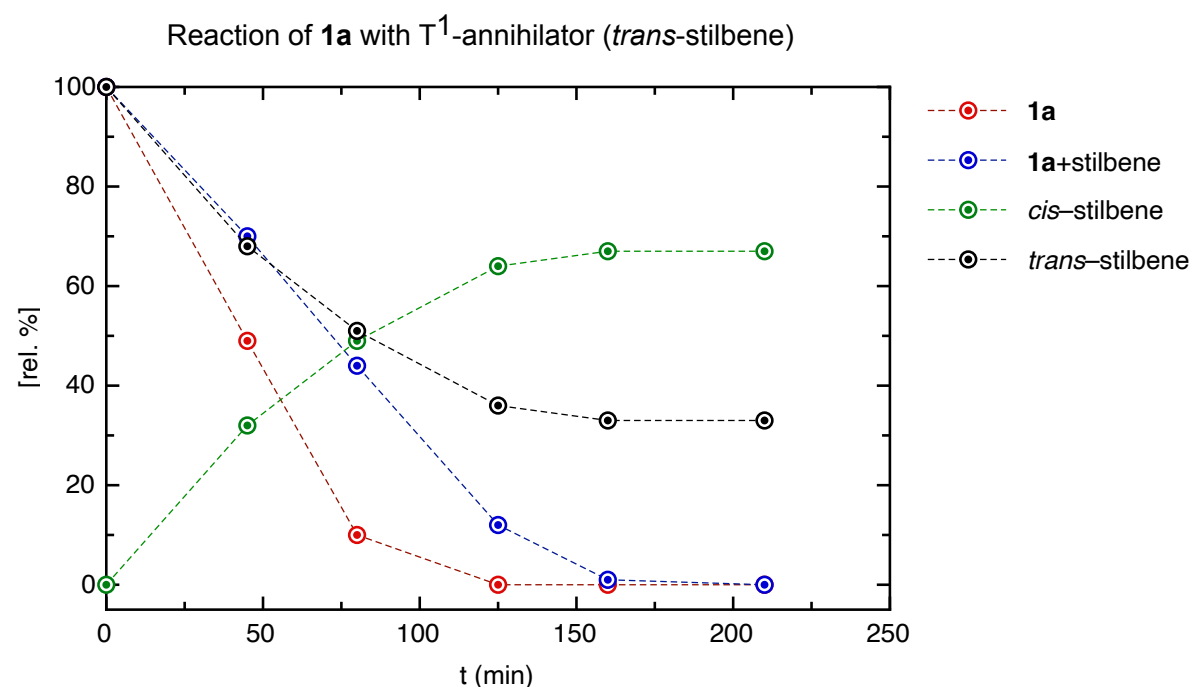

Figure SI1: Reaction progress: (a) 1a in DCM over time $(\bullet)$, (b) 1a with trans-stilbene in DCM over time $(\bullet)$, trans- to cis-stilbene conversion over time $(\bullet$ and $\bullet)$.

\subsection{Light-dark reaction}

To show that the reaction only takes place during irradiation, a reaction following the general procedure with 1a ( $32.1 \mathrm{mg}, 0.1 \mathrm{mmol})$ and DCM $(2.5 \mathrm{~mL})$ was carried out. It was consecutively either irradiated with LED $_{450 \mathrm{~nm}}$ or kept in darkness. The samples taken at regular intervals were analyzed by ${ }^{1} \mathrm{H}$ NMR spectroscopy to determine the NMR yields of $1 \mathrm{a}$. The reaction took place only during irradiation periods. 


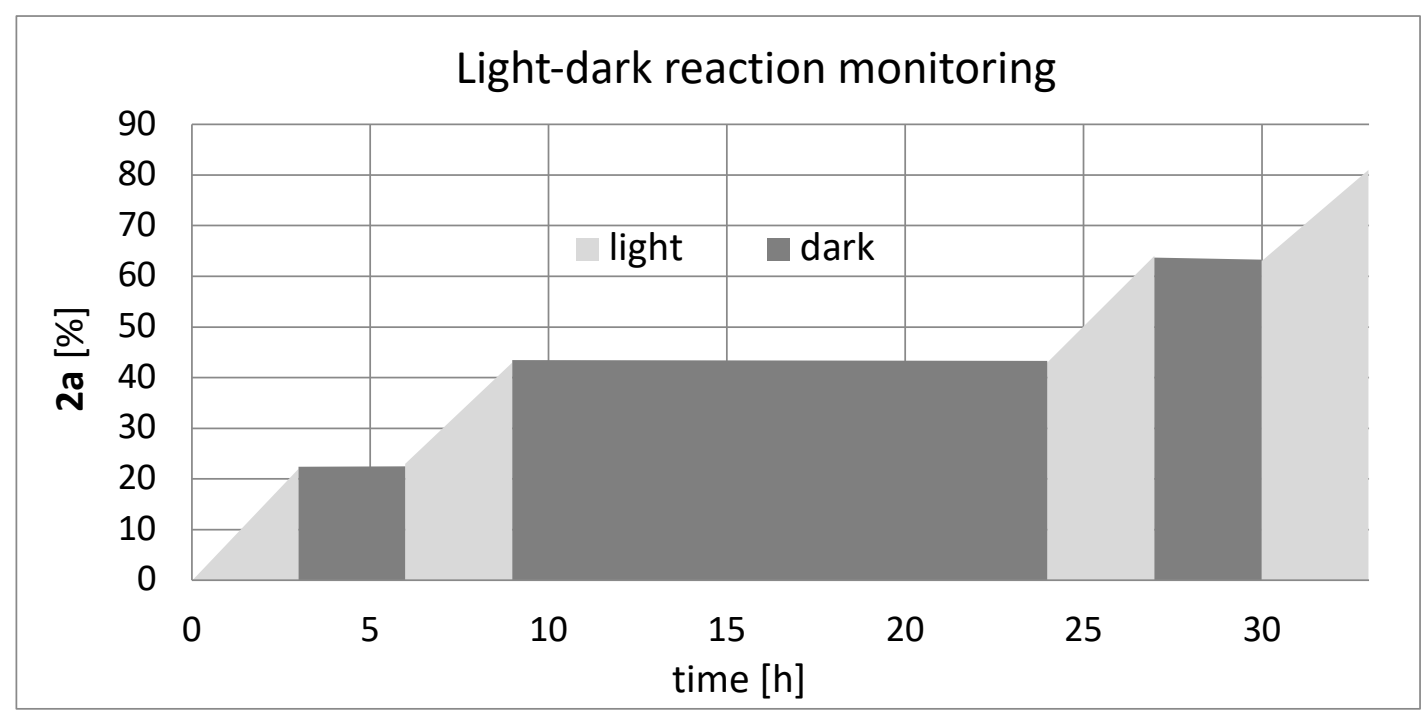

Figure SI2: Light-dark experiment for the formation of $\mathbf{2 a}$.

\subsection{Proposed mechanism for the formation of $\mathbf{3 i}$}

We propose the following reaction pathway for the formation of ring-expansion products 3 commenced via C1-N8 homolytic bond cleavage followed by radical 7-endo-trig cyclization.

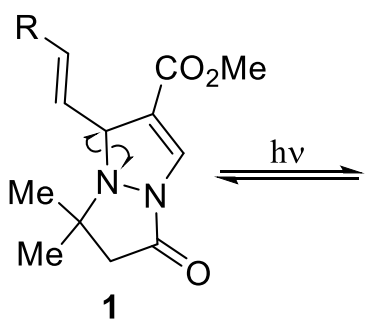

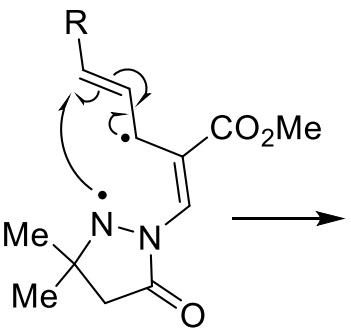

Scheme SI9: Proposed reaction pathway for the formation of compounds 3.

\subsection{Intermediate $\mathbf{1 2}$ detection}

Intermediate 12 was successfully characterized by NMR spectroscopy. A dry $8 \mathrm{~mL}$ vial was charged with 1a $(32.1 \mathrm{mg}, 0.1 \mathrm{mmol})$ and $\mathrm{CD}_{3} \mathrm{CN}(1.0 \mathrm{~mL})$. The vial was sealed off with a screw cap with a septum. The resulting solution was degassed via needle by three freeze-pump-thaw cycles. Diethyl bromomalonate ( $18.5 \mu \mathrm{L}, 92 \%$ purity, 1.0 equiv) was then added. The solution was irradiated with a blue $\mathrm{LED}_{450 \mathrm{~nm}}$ under nitrogen atmosphere for $18 \mathrm{~h}$ at $25^{\circ} \mathrm{C}$. The reaction mixture was then transferred to an NMR tube under argon. ${ }^{1} \mathrm{H}$ NMR revealed a new compound, which corresponds to intermediate 12. As the sample was then gradually exposed to moisture, the intensity of signals $\mathbf{1 2}$ decreased to which carboxylic acid $\mathbf{5 a}$ was formed. 


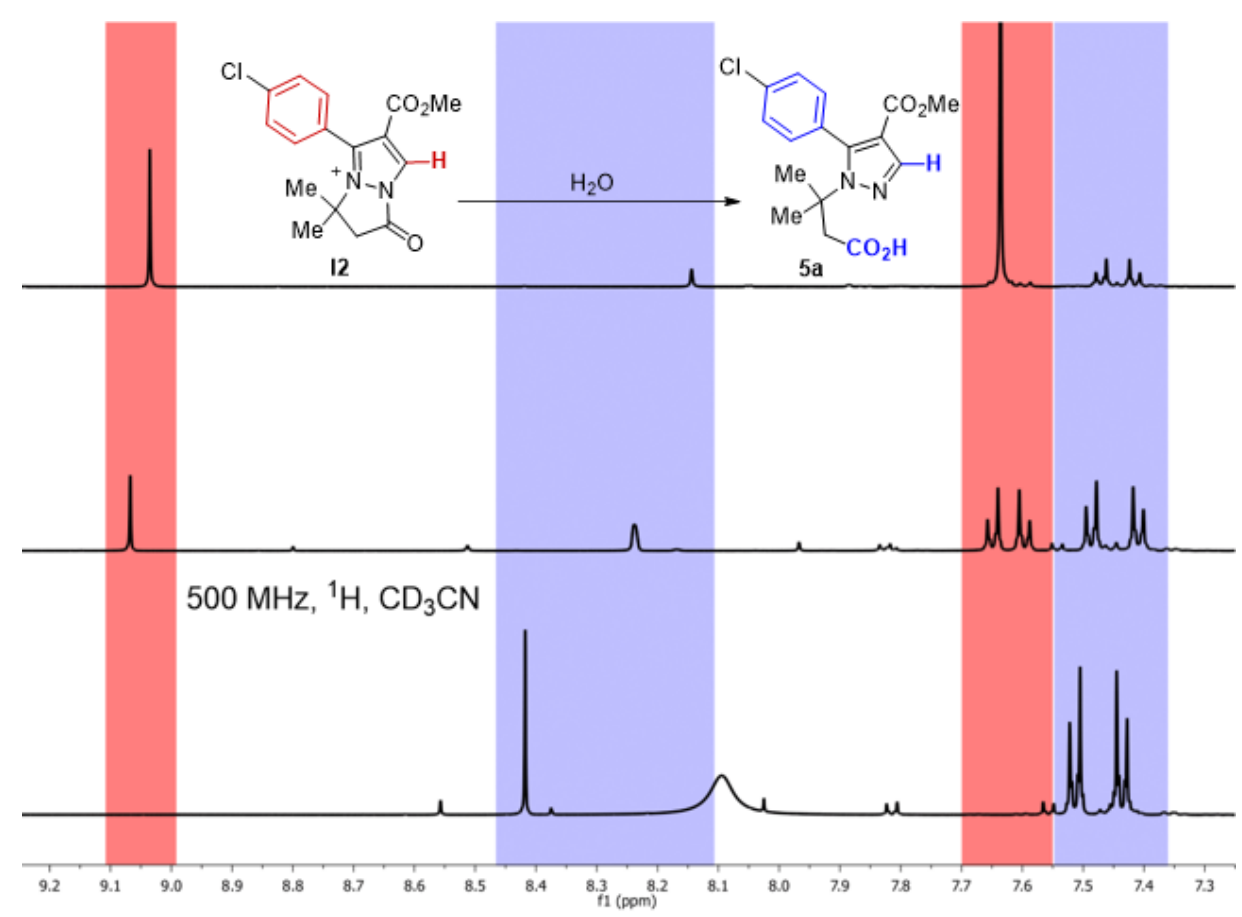

Figure SI3: ${ }^{1} \mathrm{H}$ NMR spectrum of intermediate $\mathbf{I} \mathbf{2}$ and its gradual conversion to pyrazole $\mathbf{5 a}$.

\subsection{TEMPO reaction}

A dry $8 \mathrm{~mL}$ vial was charged with 1a (161 mg, $0.5 \mathrm{mmol}$ ), TEMPO (313 mg, 4 equiv) and dry DCM $(2.5 \mathrm{~mL})$ and sealed off with a screw cap with a septum. The resulting solution was degassed via needle by three freeze-pump-thaw cycles. Diethyl bromomalonate (185 $\mu \mathrm{L}, 92 \%$ purity, 2.0 equiv) and 2,6-lutidine ( $87 \mu \mathrm{L}, 1.5$ equiv) were added. The solution was irradiated from below with blue $L_{E D} 450 \mathrm{~nm}$ under nitrogen atmosphere for $18 \mathrm{~h}$ at $25{ }^{\circ} \mathrm{C}$. HRMS of the reaction mixture confirmed the presence of a TEMPO-malonate adduct $(\mathrm{m} / \mathrm{z}=316.2115)$, which forms from a malonyl radical.

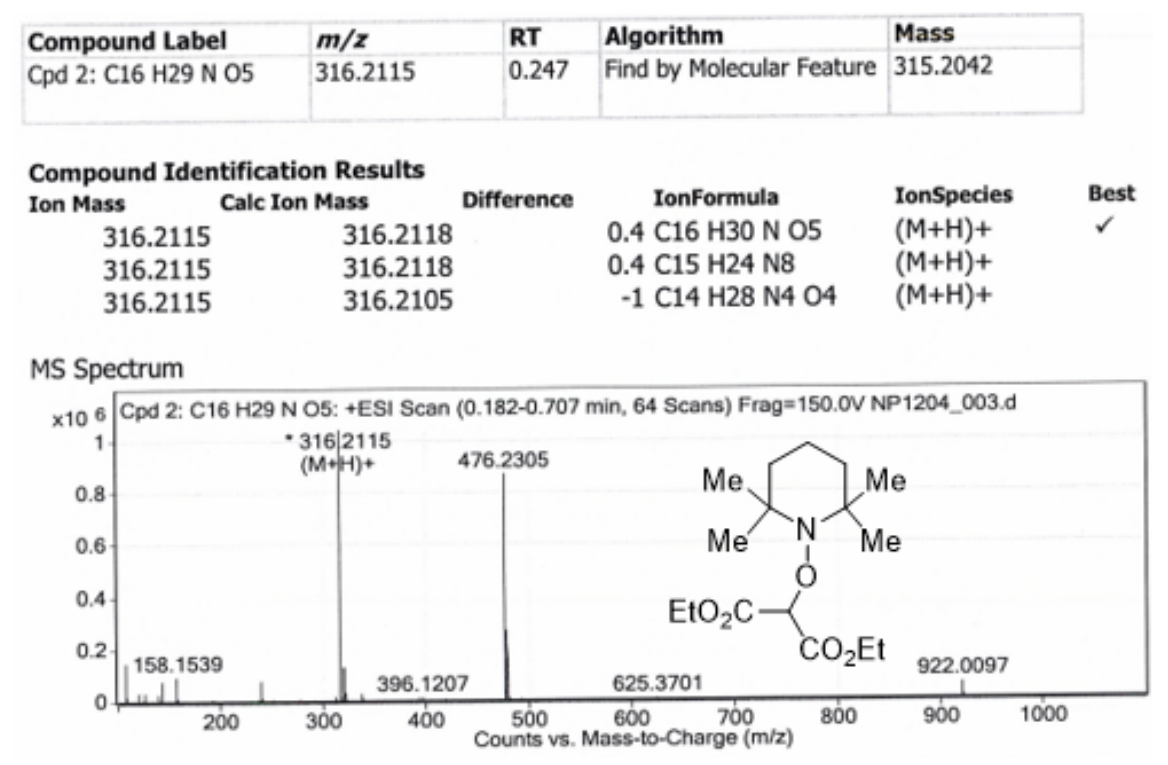

Figure SI4: MS spectrum of a reaction mixture with the TEMPO-malonate adduct. 


\section{Absorption and emission measurements}

Emission spectra of LED were measured and determined to peak at $450-455 \mathrm{~nm}$ for blue and $510-515 \mathrm{~nm}$ for green LED.

\section{LED Emission spectra}

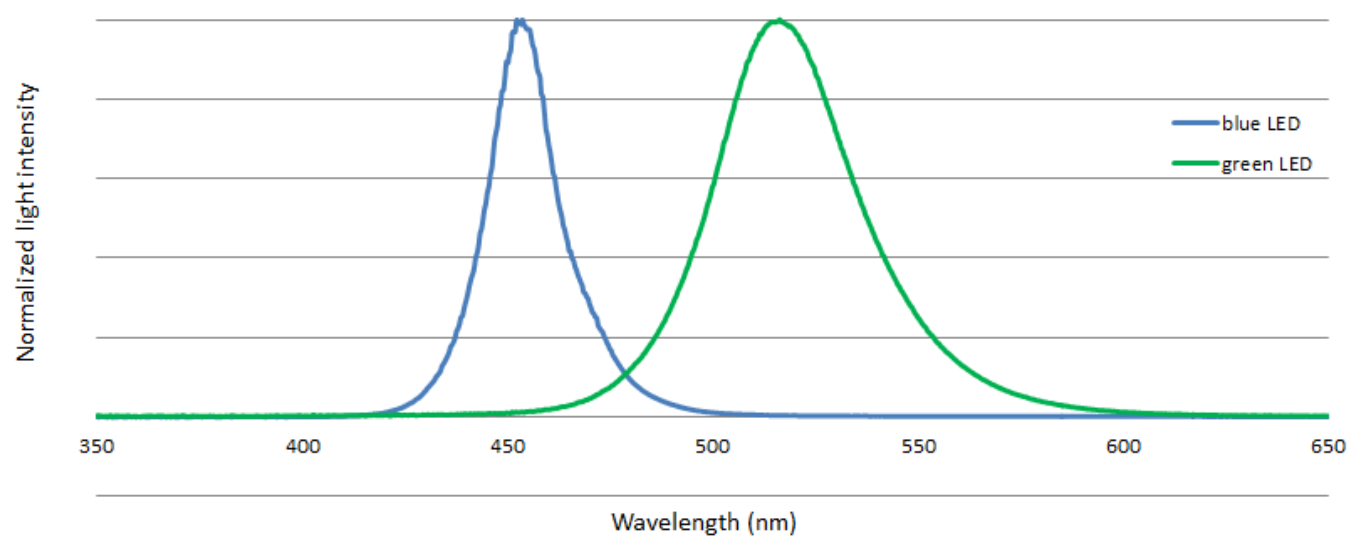

Figure SI5: Emission spectra of blue and green LED.

Absorption spectra of $3 \mu \mathrm{M}$ solutions of 1 in DCM were measured. The absorption profile that trails into the visible region (around $400 \mathrm{~nm}$ ) differs little between compounds. The extinction coefficient for compound 1a was also measured in $\operatorname{MeCN}\left(\varepsilon=8400 \mathrm{M}^{-1} \mathrm{~cm}^{-1}\right)$.

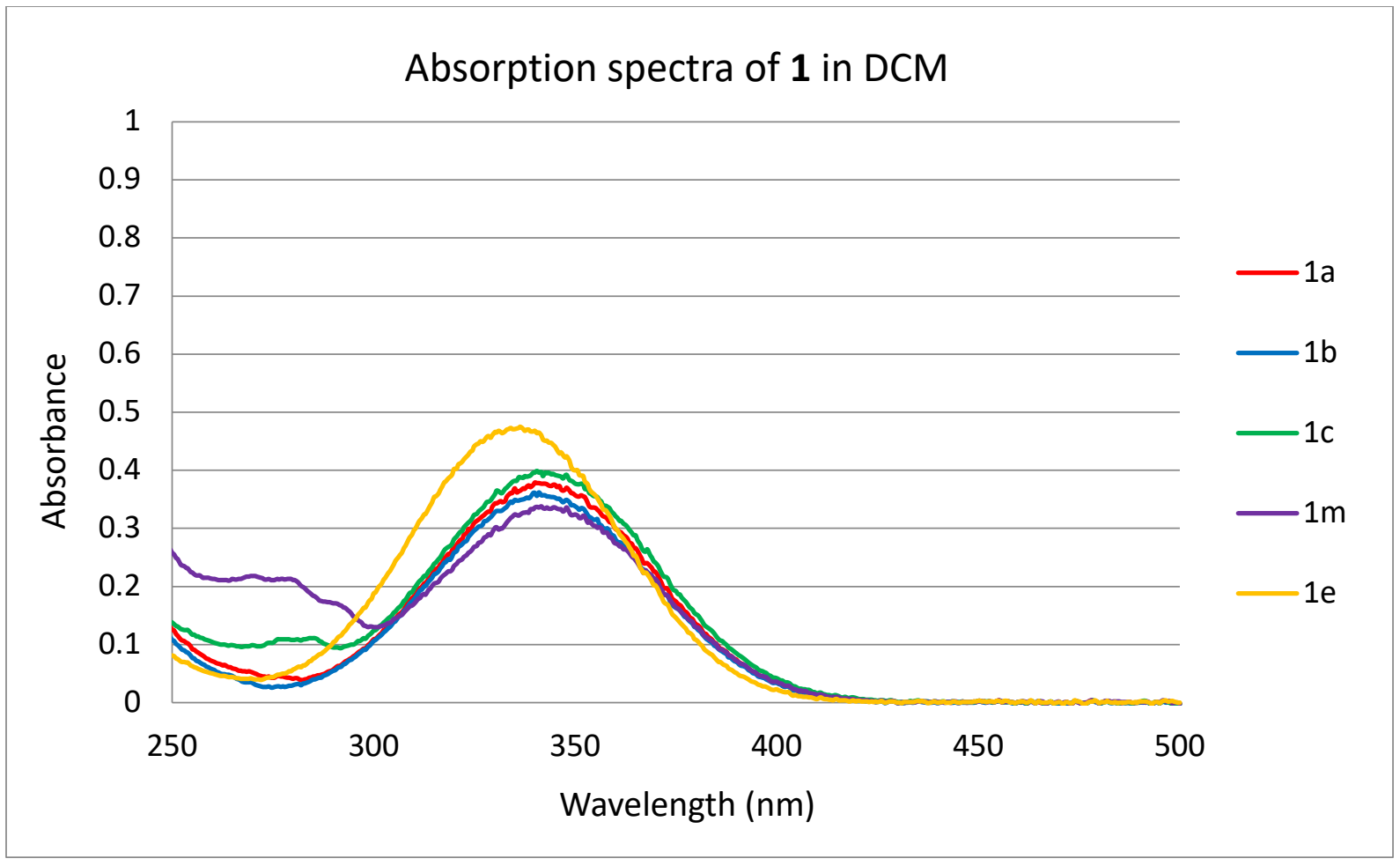

Figure SI6: Absorption spectra of compounds 1. 


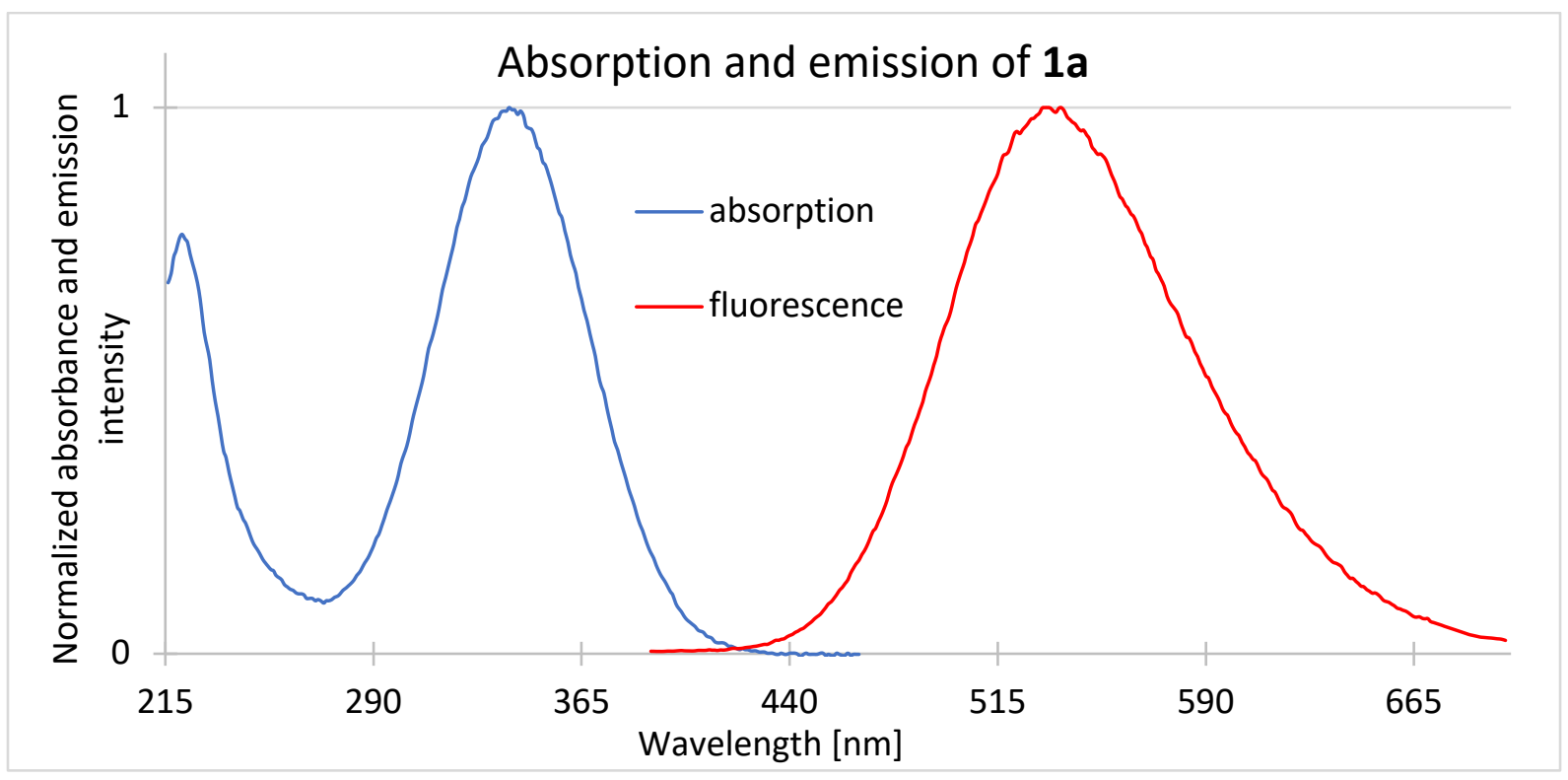

Figure SI7: Absorption and emission spectra of compound 1a.

Fluorescence quantum yield of compound 1a in $\mathrm{MeCN}$ was determined from the ratio of integrated fluorescence intensity and absorbance. ${ }^{15}$ 9-Fluorenone was chosen as a reference with fluorescence quantum yield of $2.7 \%$ in $\mathrm{MeCN} .{ }^{16}$

$\Phi_{f}=2.7 \% \times 2.48 / 0.59=11.3 \%$

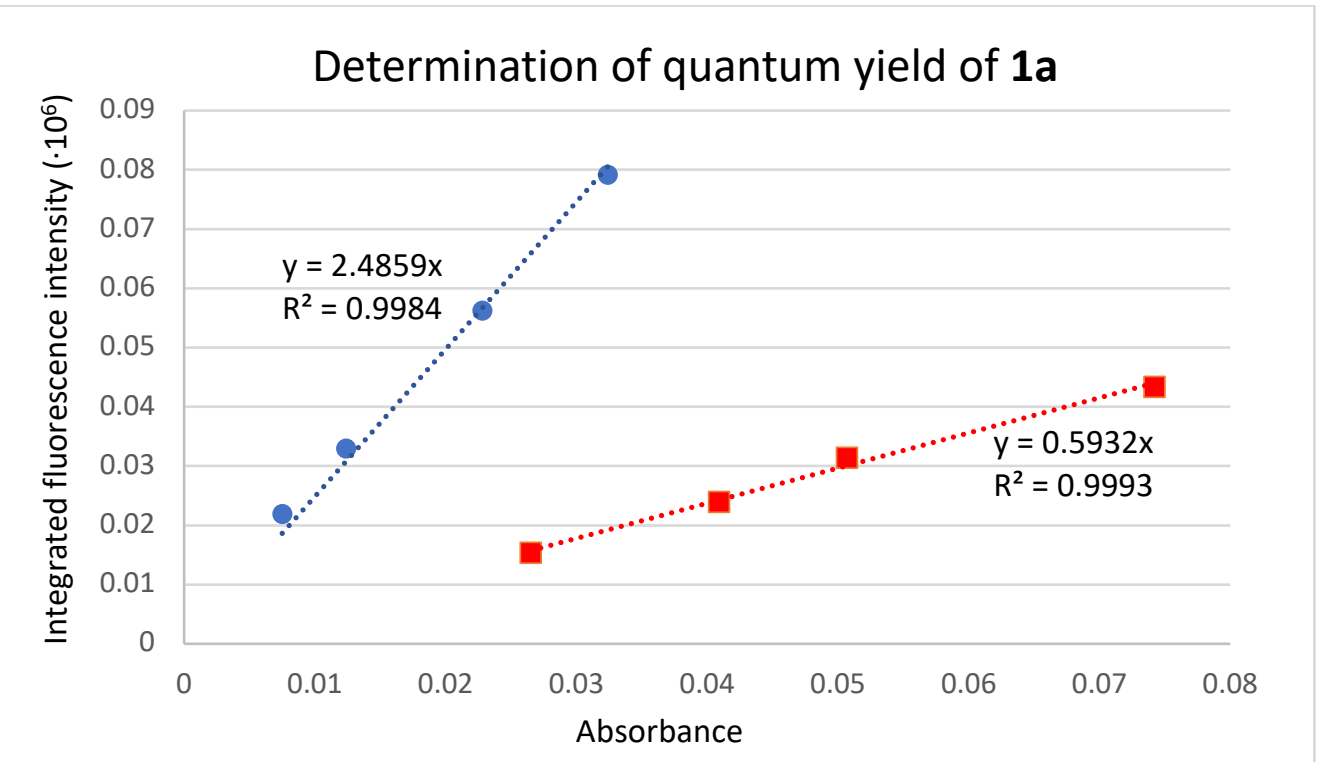

Figure S18: Plot of integrated fluorescence intensity against absorbance for 9-fluorenone and compound 1a ( $\bullet$ 1a, $\mathbf{a}$ fluorenone).

\section{Stern-Volmer quenching experiments}

A $20 \mu \mathrm{M}$ solution of $1 \mathrm{a}$ in $\mathrm{MeCN}$ was degassed and fluorescence of the solution measured under nitrogen atmosphere at different concentrations of diethyl bromomalonate. Fluorescence intensity decreased with increased concentration of diethyl malonate in the solution, which indicates its interaction with 1a*. 


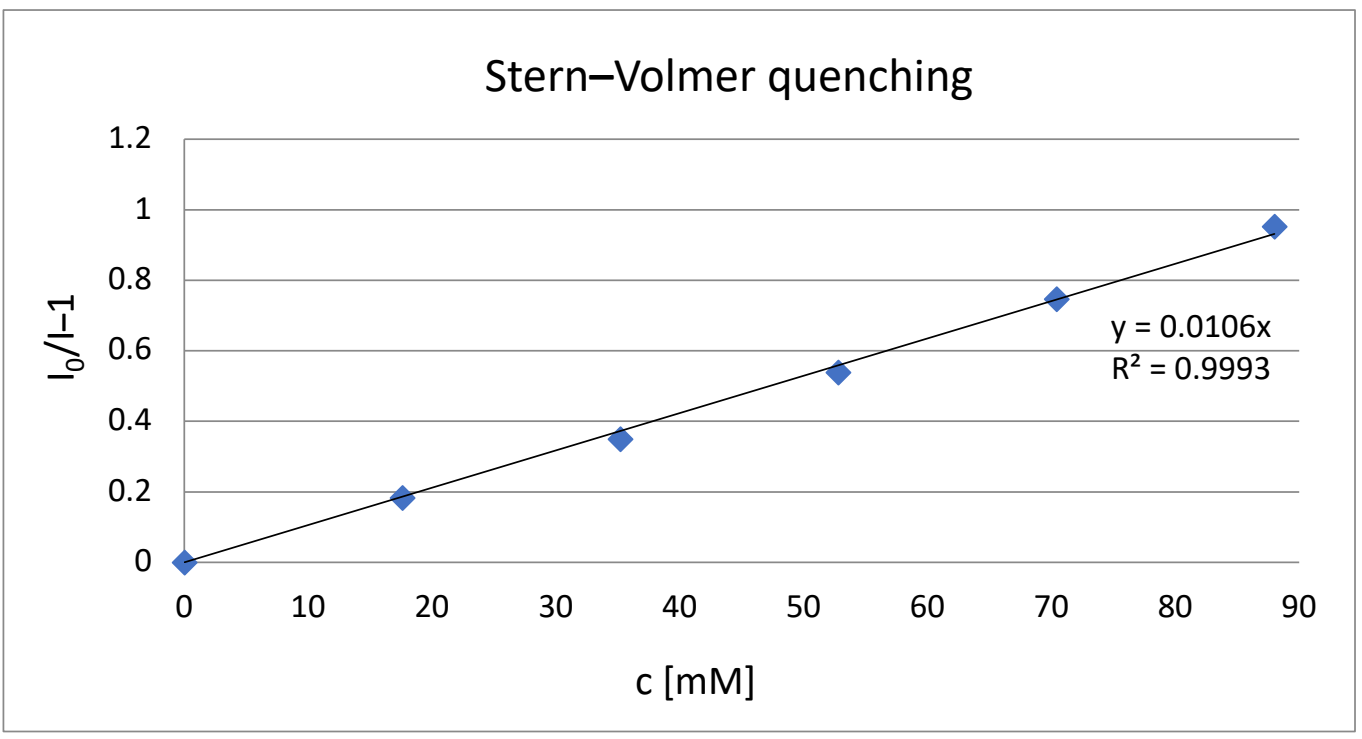

Figure SI9: Stern-Volmer plot of 1a quenching by diethyl bromomalonate.

\section{Cyclic Voltammograms}

A cyclic voltammogram of compound 1a was recorded. A solution of $1 \mathrm{a}(10 \mathrm{mM})$ was prepared in $\mathrm{MeCN}_{\text {with }} \mathrm{Bu}_{4} \mathrm{NBF}_{4}(50 \mathrm{mM})$ as an electrolyte. Ferrocene was added as an internal standard. Applied potential was increased from $0 \mathrm{~V}$ to $2 \mathrm{~V}$ and back to $0 \mathrm{~V}$ with a scan rate of $10 \mathrm{mV} / \mathrm{s}$.

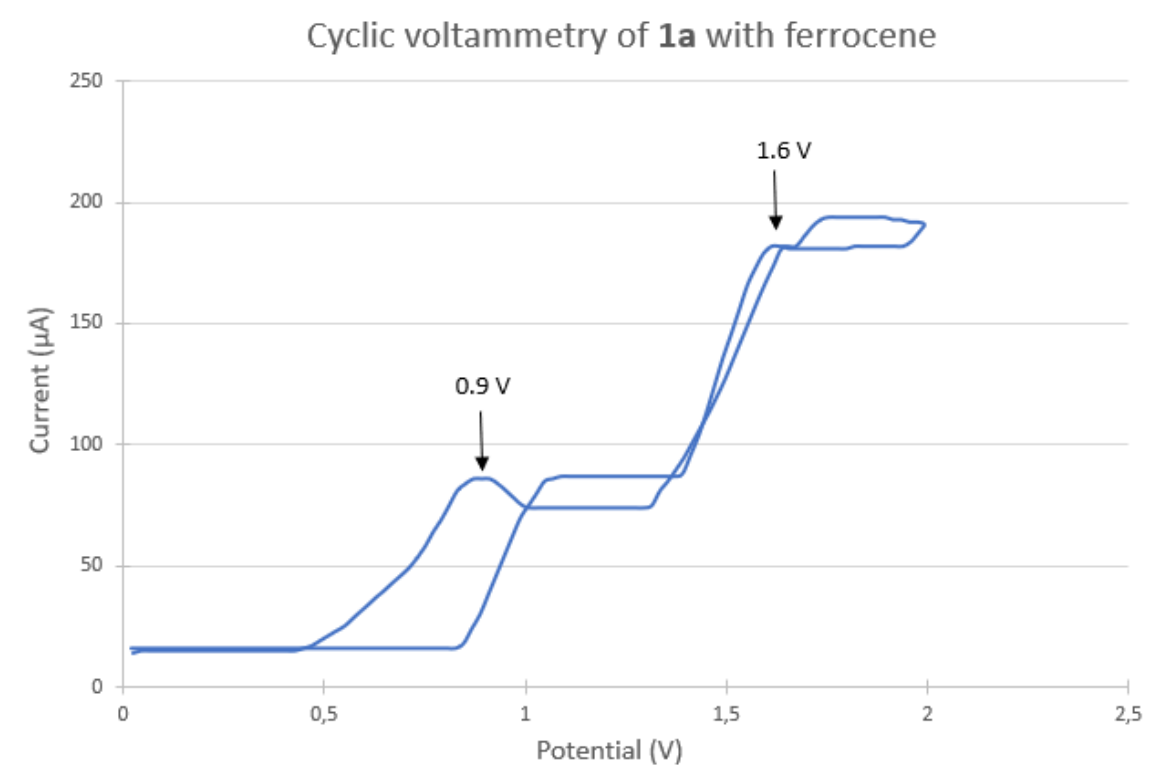

Figure SI10: CV plot of compound 1a with ferrocene.

$E_{1 / 2}\left(\mathrm{Fc}^{+} / \mathrm{Fc}\right)=0.4 \mathrm{~V}(\mathrm{vs} . \mathrm{SCE})^{17}$

$E_{1 / 2}\left(1 a^{\cdot+/ 1 a}\right)=1.1 \vee(v s . S C E)$

From the absorption and emission spectra, we can write:

$E_{0,0} * 423 \mathrm{~nm}=2.93 \mathrm{eV}$

$E_{1 / 2}\left(1 a^{+} / 1 a^{*}\right)=1.1 \mathrm{~V}-2.9 \vee($ vs. SCE $)=-1.8 \vee(v s . ~ S C E)$ 


\section{X-ray crystallography data}

Crystals were prepared by slow evaporation of DCM solutions at room temperature. Singlecrystal X-ray diffraction data was collected on Agilent Technologies SuperNova Dual diffractometer with an Atlas detector using monochromated Mo- $K_{\alpha}$ radiation $(\lambda=0.71073 \AA)$ at $293 \mathrm{~K}$. The data was processed using CrysAlis $\mathrm{PRO}^{18}$. Using Olex2.1.2. ${ }^{19}$, the structures were solved by direct methods implemented in SHELXS ${ }^{20}$ or SHELXT ${ }^{21}$ and refined by a full-matrix least-squares procedure based on $\mathrm{F}^{2}$ with SHELXT-2014/722. All nonhydrogen atoms were refined anisotropicallly. Hydrogen atoms were placed in geometrically calculated positions and were refined using a riding model. The drawings and the analysis of bond lengths, angles and intermolecular interactions were carried out using Mercury ${ }^{23}$ and Platon ${ }^{24}$.

Table SI3: Crystal data and structure refinement for compounds $\mathbf{2 c}$, $\mathbf{3 i}$ and $\mathbf{4 b}$.

\begin{tabular}{|c|c|c|c|}
\hline Compound & $2 c$ & $3 \mathbf{i}$ & $4 b$ \\
\hline Empirical formula & $\mathrm{C}_{17} \mathrm{H}_{20} \mathrm{~N}_{2} \mathrm{O}_{4}$ & $\mathrm{C}_{18} \mathrm{H}_{20} \mathrm{~N}_{2} \mathrm{O}_{3}$ & $\mathrm{C}_{16} \mathrm{H}_{16} \mathrm{~N}_{2} \mathrm{O}_{3}$ \\
\hline Formula weight & 316.35 & 312.36 & 284.31 \\
\hline Temperature/K & $150.00(10)$ & $150.00(10)$ & 293(2) \\
\hline Crystal system & triclinic & monoclinic & monoclinic \\
\hline Space group & P-1 & $\mathrm{P} 2{ }_{1} / \mathrm{n}$ & $\mathrm{P} 22_{1} / \mathrm{n}$ \\
\hline$a\left[\AA^{3}\right]$ & $9.4508(7)$ & $9.3608(4)$ & $12.3566(6)$ \\
\hline$b\left[\AA^{3}\right]$ & $10.0870(8)$ & $14.3093(5)$ & $9.3671(4)$ \\
\hline$c\left[\AA^{3}\right]$ & $10.1375(7)$ & $12.5589(5)$ & $12.8560(6)$ \\
\hline$\alpha\left[^{\circ}\right]$ & $62.823(7)$ & 90 & 90 \\
\hline$B\left[^{\circ}\right]$ & $70.139(7)$ & $105.553(4)$ & $106.093(5)$ \\
\hline$\gamma\left[{ }^{\circ}\right]$ & $76.003(6)$ & 90 & 90 \\
\hline$V\left[\AA^{3}\right]$ & $804.23(12)$ & $1620.62(11)$ & $1429.71(12)$ \\
\hline Z & 2 & 4 & 4 \\
\hline$\rho_{\text {calc }}\left[\mathrm{g} / \mathrm{cm}^{3}\right]$ & 1.306 & 1.280 & 1.321 \\
\hline$\mu\left[\mathrm{mm}^{-1}\right]$ & 0.094 & 0.088 & 0.093 \\
\hline$F(000)$ & 336.0 & 664.0 & 600.0 \\
\hline Crystal size/mm $\mathrm{mm}^{3}$ & $0.5 \times 0.4 \times 0.2$ & $0.4 \times 0.3 \times 0.2$ & $0.5 \times 0.4 \times 0.2$ \\
\hline Radiation & $\operatorname{MoK} \alpha(\lambda=0.71073)$ & $\operatorname{MoK} \alpha(\lambda=0.71073)$ & $\operatorname{MoK} \alpha(\lambda=0.71073)$ \\
\hline Reflections collected & 7562 & 15235 & 12418 \\
\hline Independent reflections & 4148 & 4401 & 3848 \\
\hline$R_{\text {int }}$ & 0.0293 & 0.0323 & 0.0297 \\
\hline Data/restraints/parameters & $4148 / 0 / 213$ & $4401 / 0 / 212$ & $3848 / 0 / 194$ \\
\hline GOF & 1.064 & 1.021 & 1.027 \\
\hline$R_{1}, w R_{2}[\mathrm{I}>=2 \sigma(\mathrm{I})]$ & $0.0489,0.1056$ & $0.0431,0.1005$ & $0.0416,0.1023$ \\
\hline$R_{1}, w R_{2}$ (all data) & $0.0726,0.1265$ & $0.0595,0.1106$ & $0.0553,0.1125$ \\
\hline$(\Delta \rho)_{\max }\left[\mathrm{e} \AA^{-3}\right]$ & 0.27 & 0.29 & 0.30 \\
\hline$(\Delta \rho)_{\min }\left[\mathrm{e} \AA^{-3}\right]$ & -0.22 & -0.21 & -0.21 \\
\hline
\end{tabular}




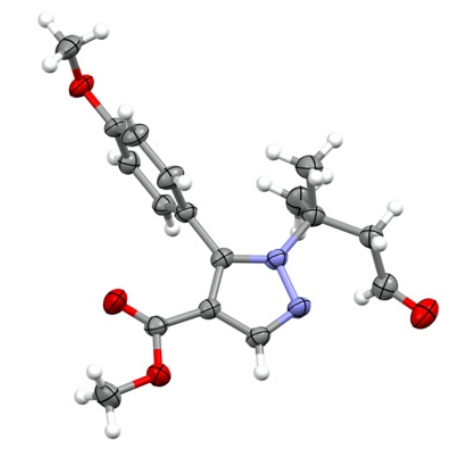

Figure SI11: Molecular structure of product 2c. Thermal ellipsoids are shown at $50 \%$ probability. CCDC number: 2077376

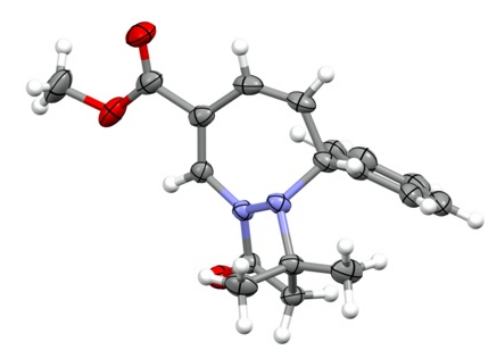

Figure SI12: Molecular structure of product 3i. Thermal ellipsoids are shown at $50 \%$ probability. CCDC number: 2077377

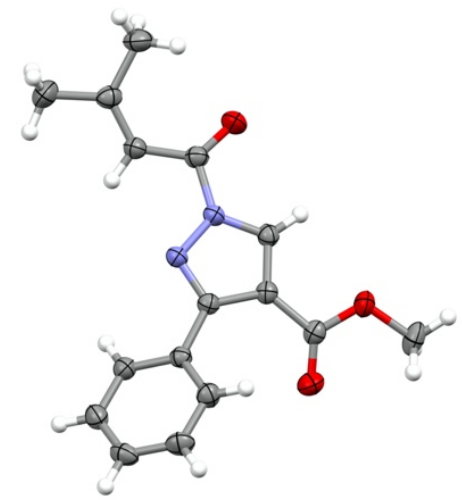

Figure SI13: Molecular structure of product $\mathbf{4 b}$. Thermal ellipsoids are shown at $50 \%$ probability. CCDC number: 2077375 


\section{NMR spectra}

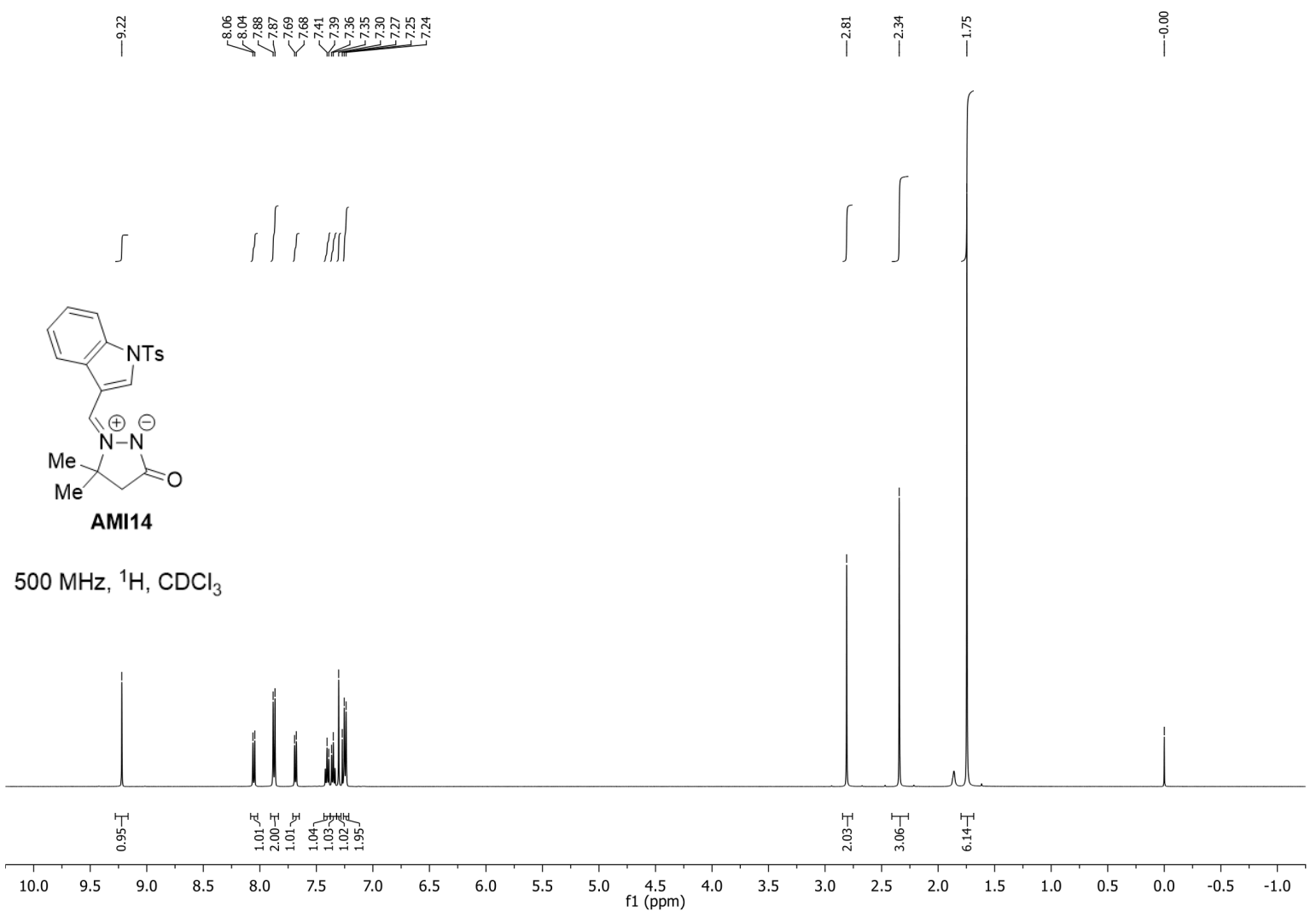

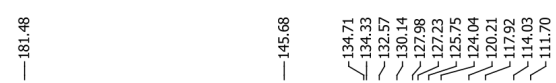
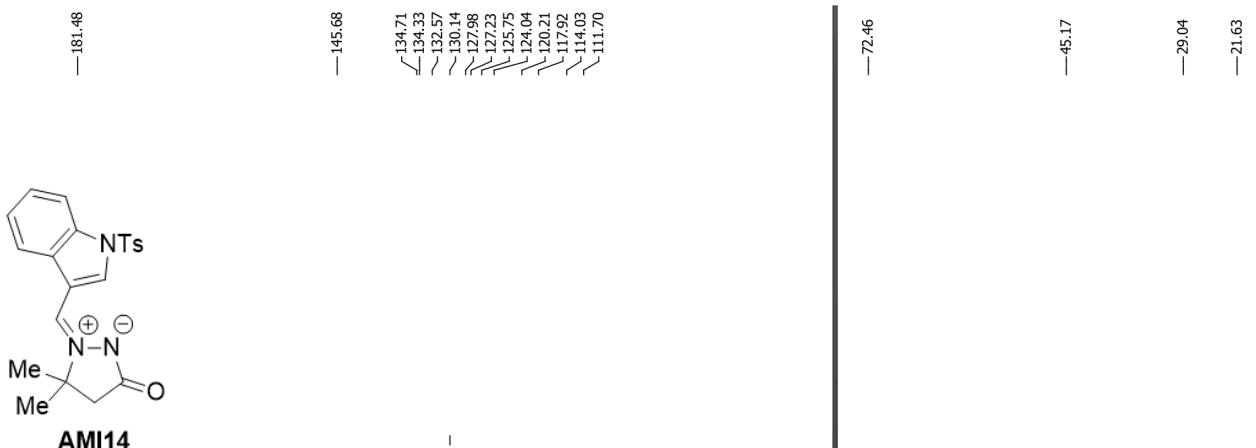

$126 \mathrm{MHz},{ }^{13} \mathrm{C}, \mathrm{CDCl}_{3}$

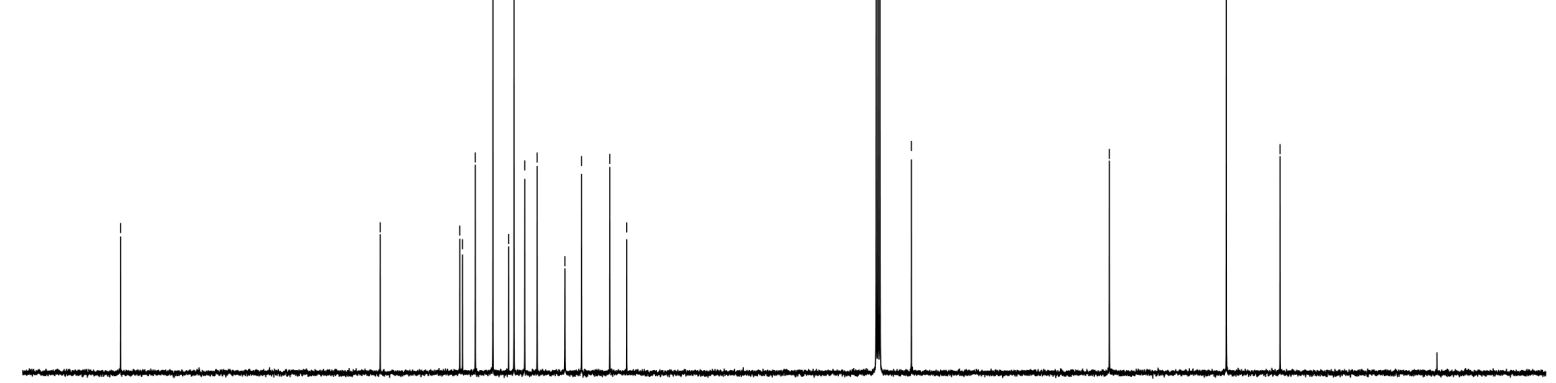

$\begin{array}{lllllllllll}190 & 180 & 170 & 160 & 150 & 140 & 130 & 120 & 110 & 100 & 90 \\ \mathrm{f} 1(\mathrm{ppm})\end{array}$ 


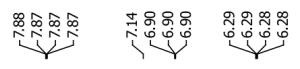

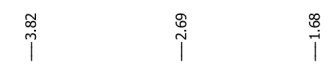

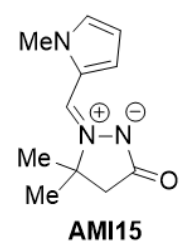

$500 \mathrm{MHz},{ }^{1} \mathrm{H}, \mathrm{CDCl}_{3}$

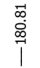

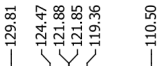

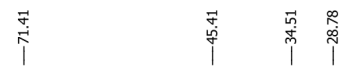

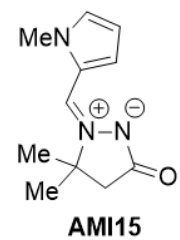

$126 \mathrm{MHz},{ }^{13} \mathrm{C}, \mathrm{CDCl}_{3}$

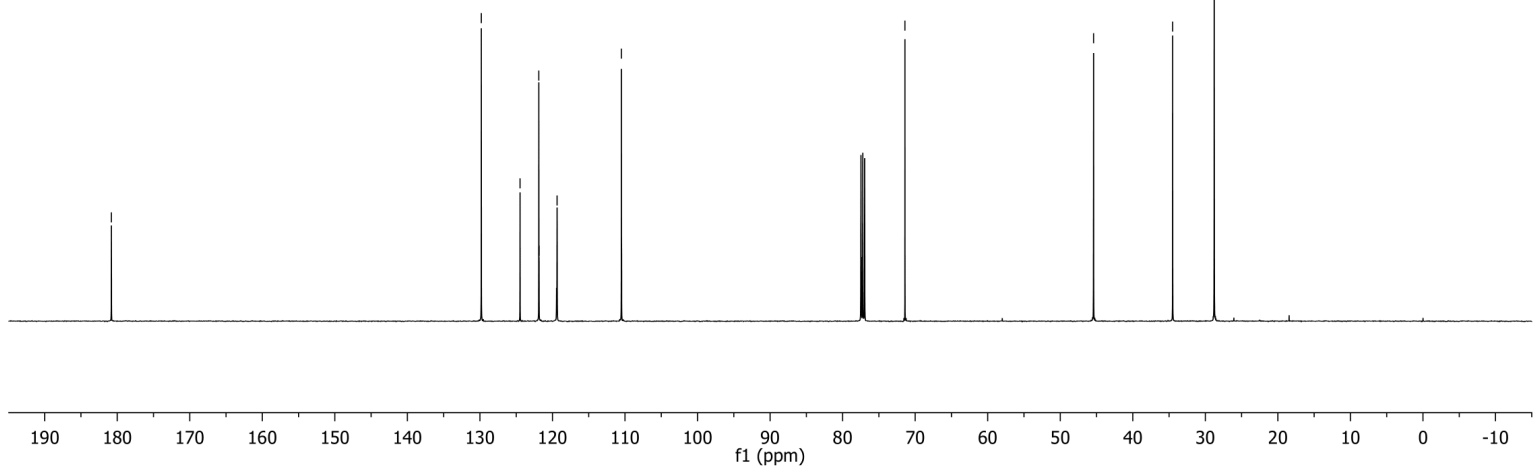




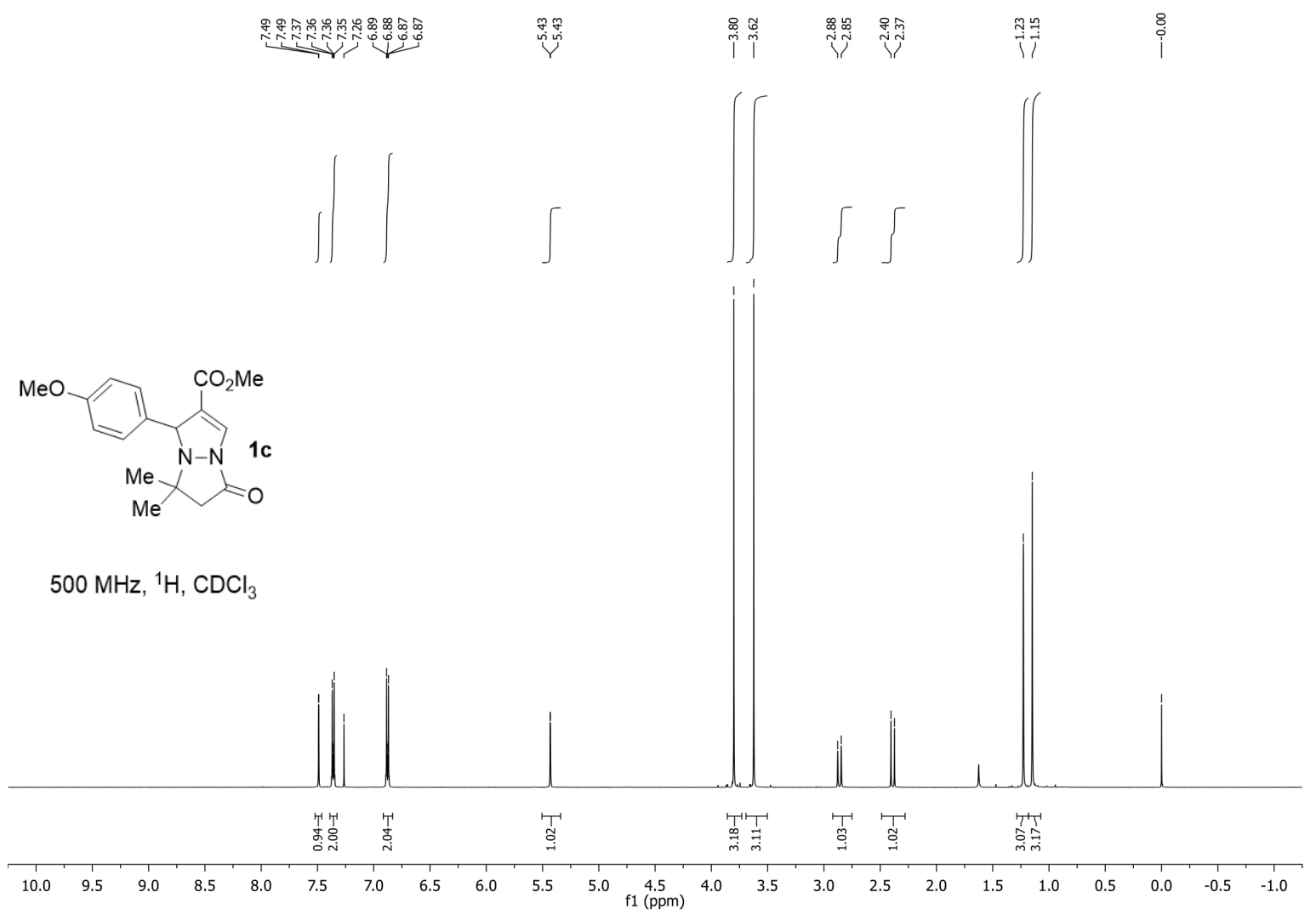

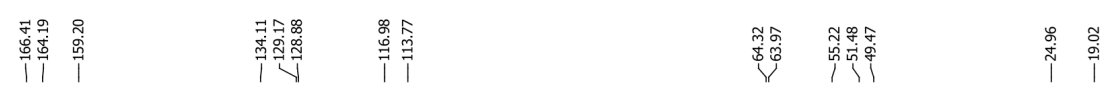

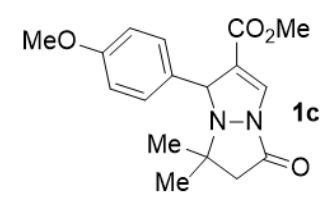

$126 \mathrm{MHz},{ }^{13} \mathrm{C}, \mathrm{CDCl}_{3}$

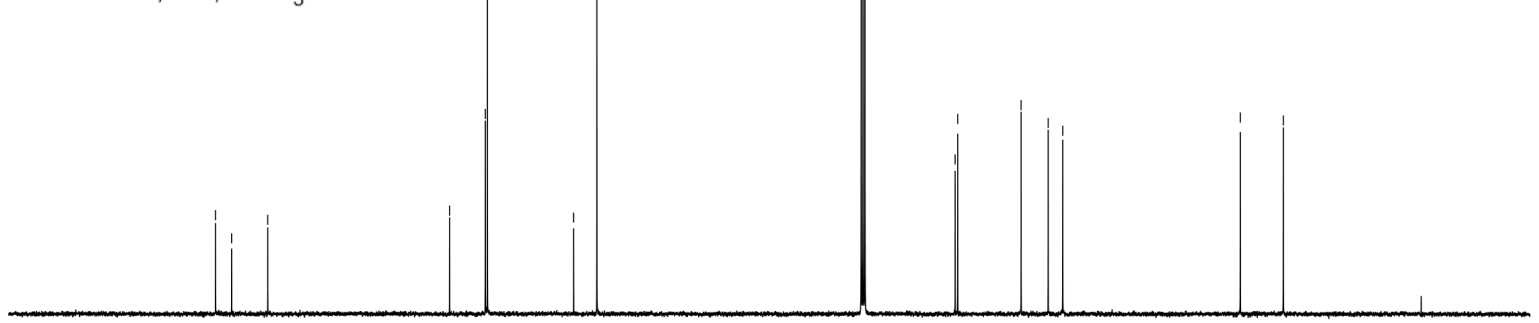

$\begin{array}{llllllllllllllllllllll}190 & 180 & 170 & 160 & 150 & 140 & 130 & 120 & 110 & 100 & 90 & 80 & 70 & 60 & 50 & 40 & 30 & 20 & 10 & 0 & -10\end{array}$ 


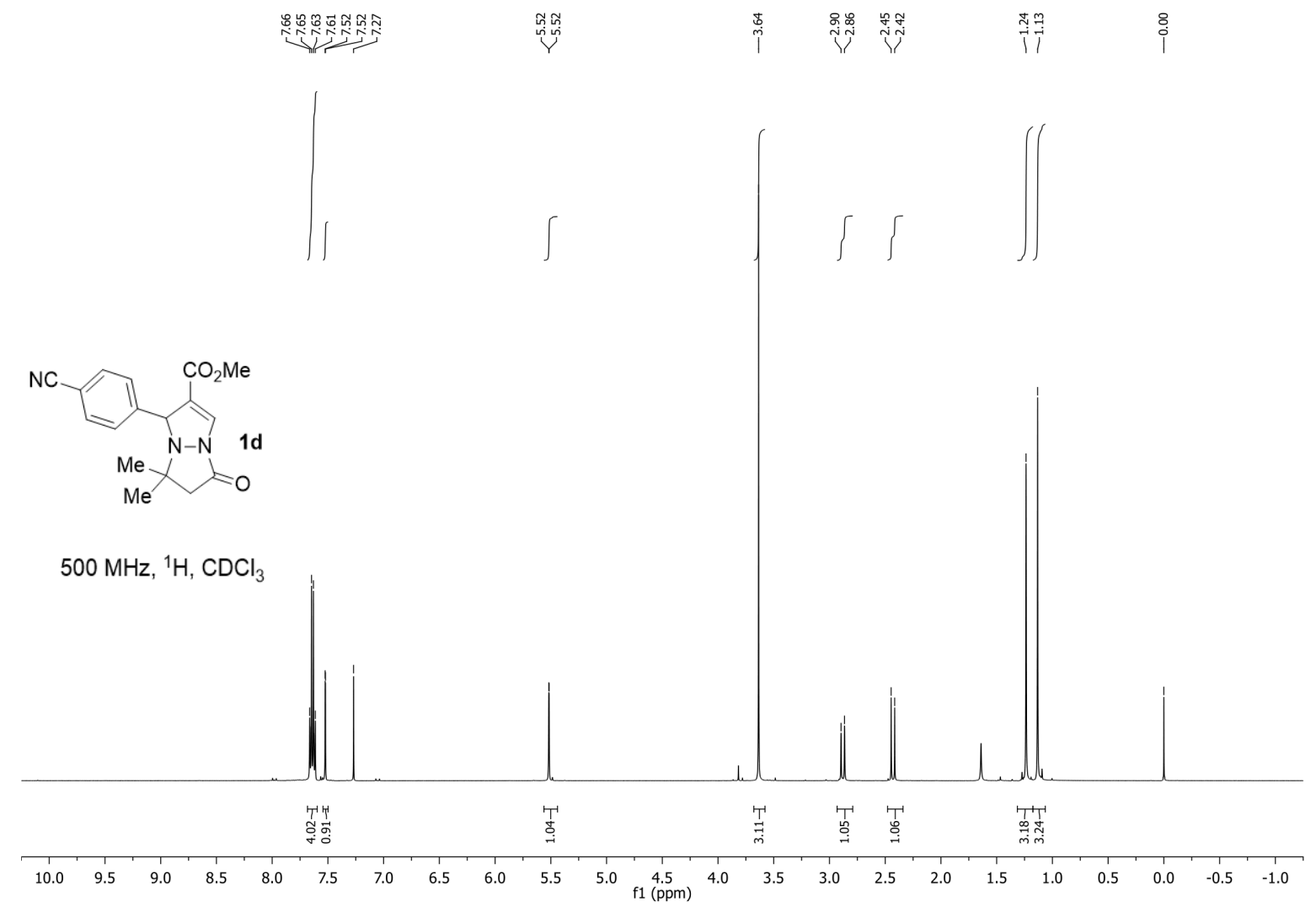

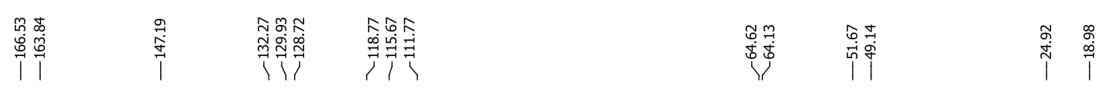

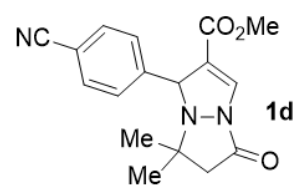

$126 \mathrm{MHz},{ }^{13} \mathrm{C}, \mathrm{CDCl}_{3}$

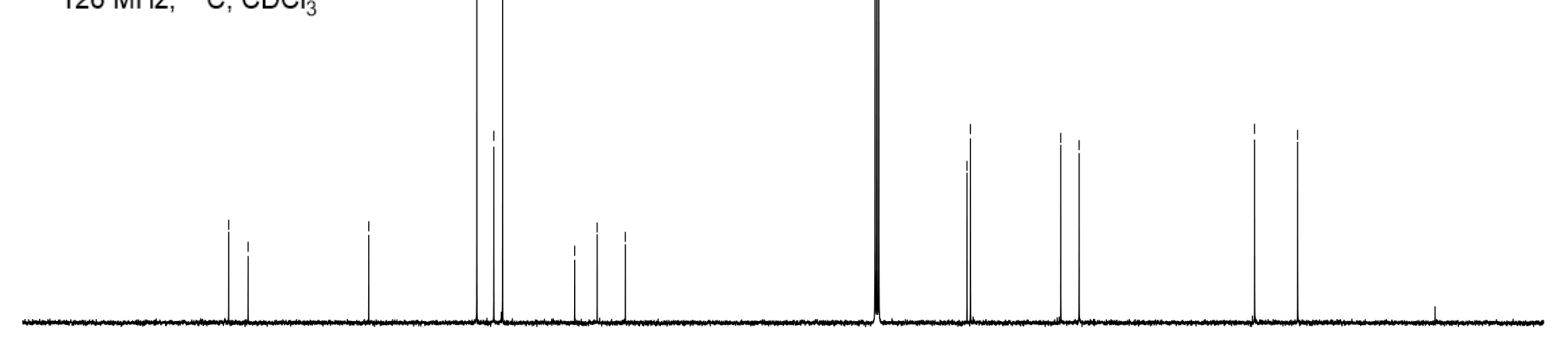

$\begin{array}{llllllllllllllllllllllll}190 & 180 & 170 & 160 & 150 & 140 & 130 & 120 & 110 & 100 & 90 & 80 & 70 & 60 & 50 & 40 & 30 & 20 & 10 & 0 & -10\end{array}$ 


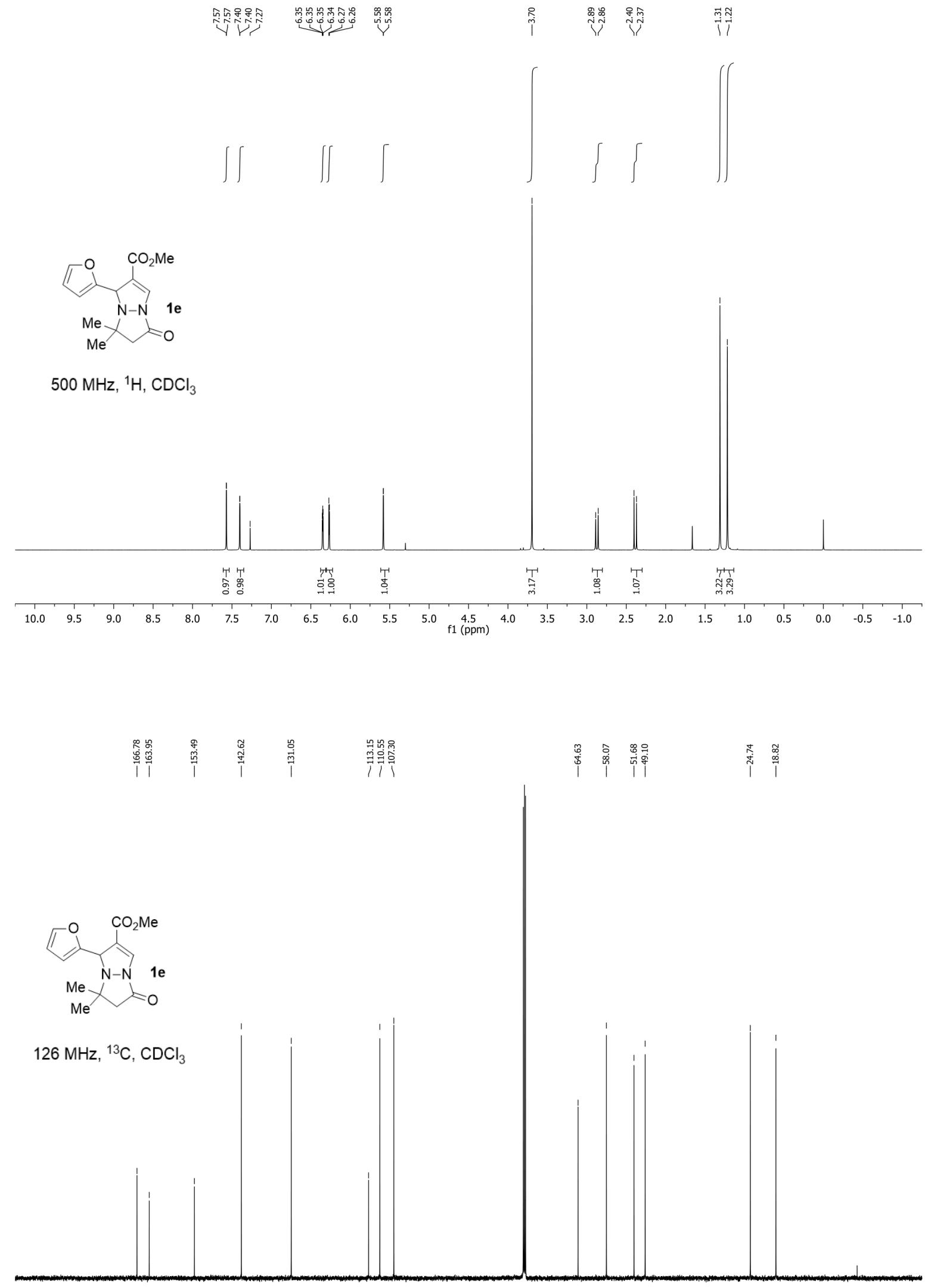

$\begin{array}{lllllllllllllllllllll}190 & 180 & 170 & 160 & 150 & 140 & 130 & 120 & 110 & 100 & 90 & 80 & 70 & 60 & 50 & 40 & 30 & 20 & 10 & 0 & -10\end{array}$ 

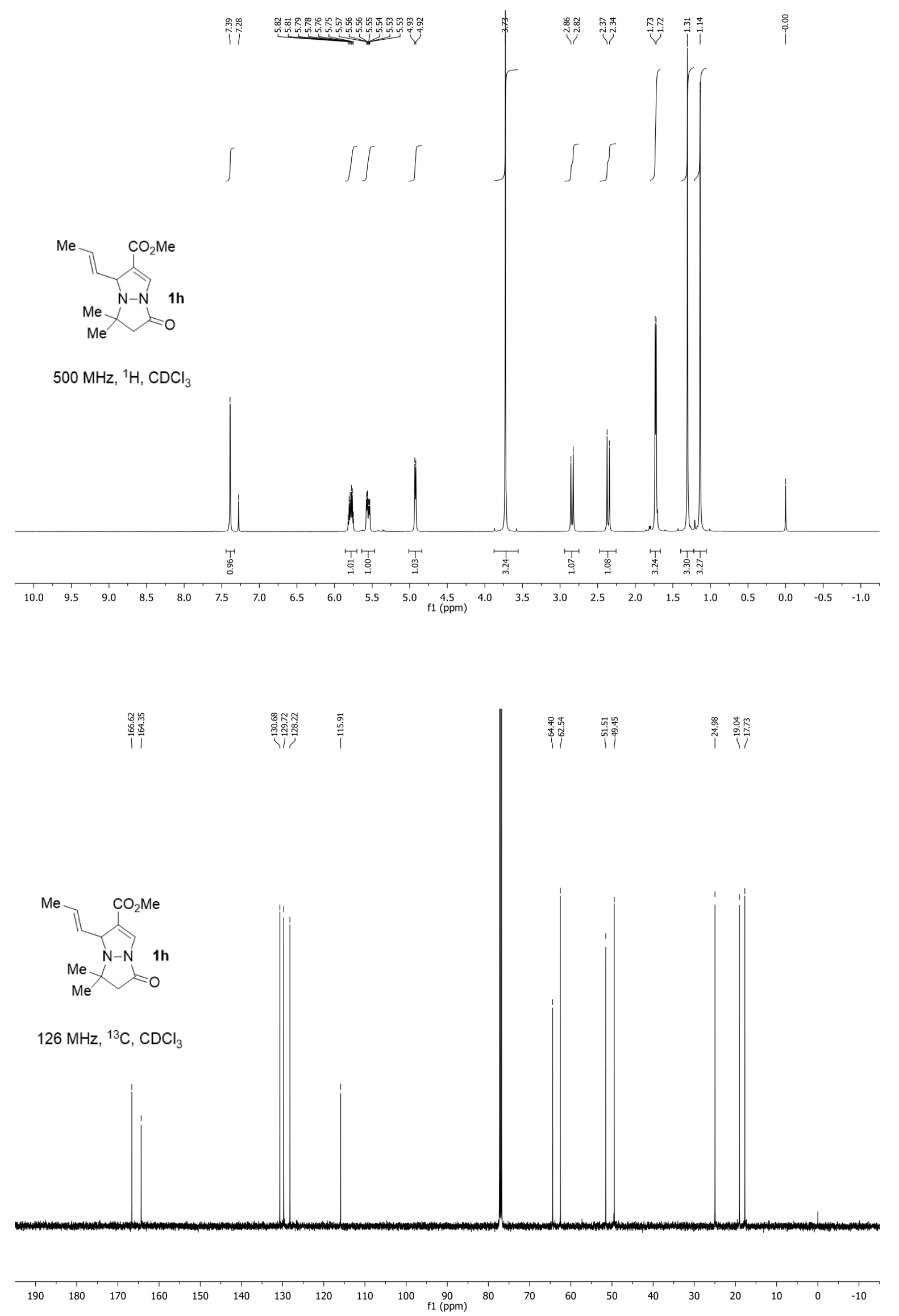

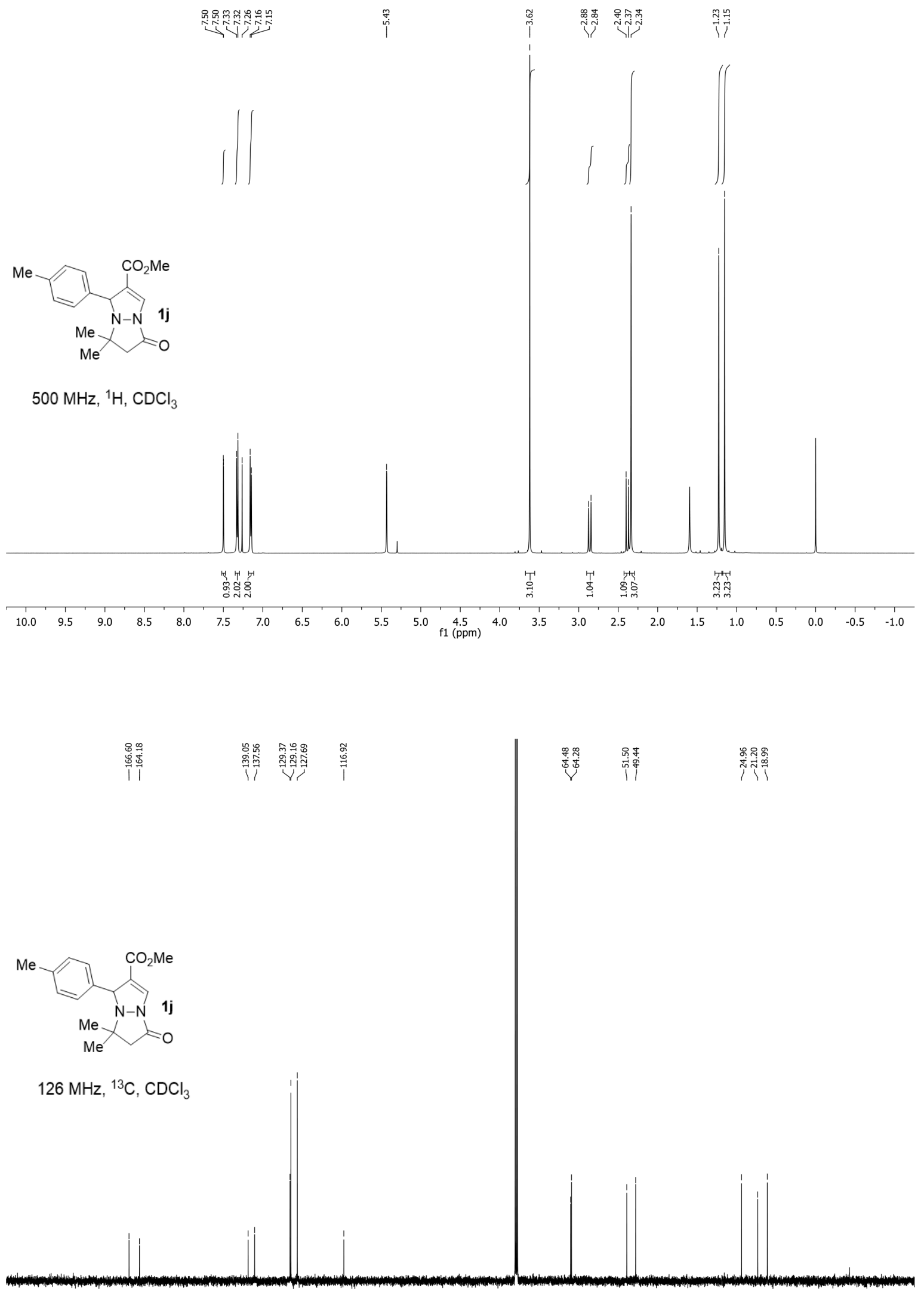

$\begin{array}{lllllllllllllllllllllll}190 & 180 & 170 & 160 & 150 & 140 & 130 & 120 & 110 & 100 & 90 & 80 & 70 & 60 & 50 & 40 & 30 & 20 & 10 & 0 & -10\end{array}$ 

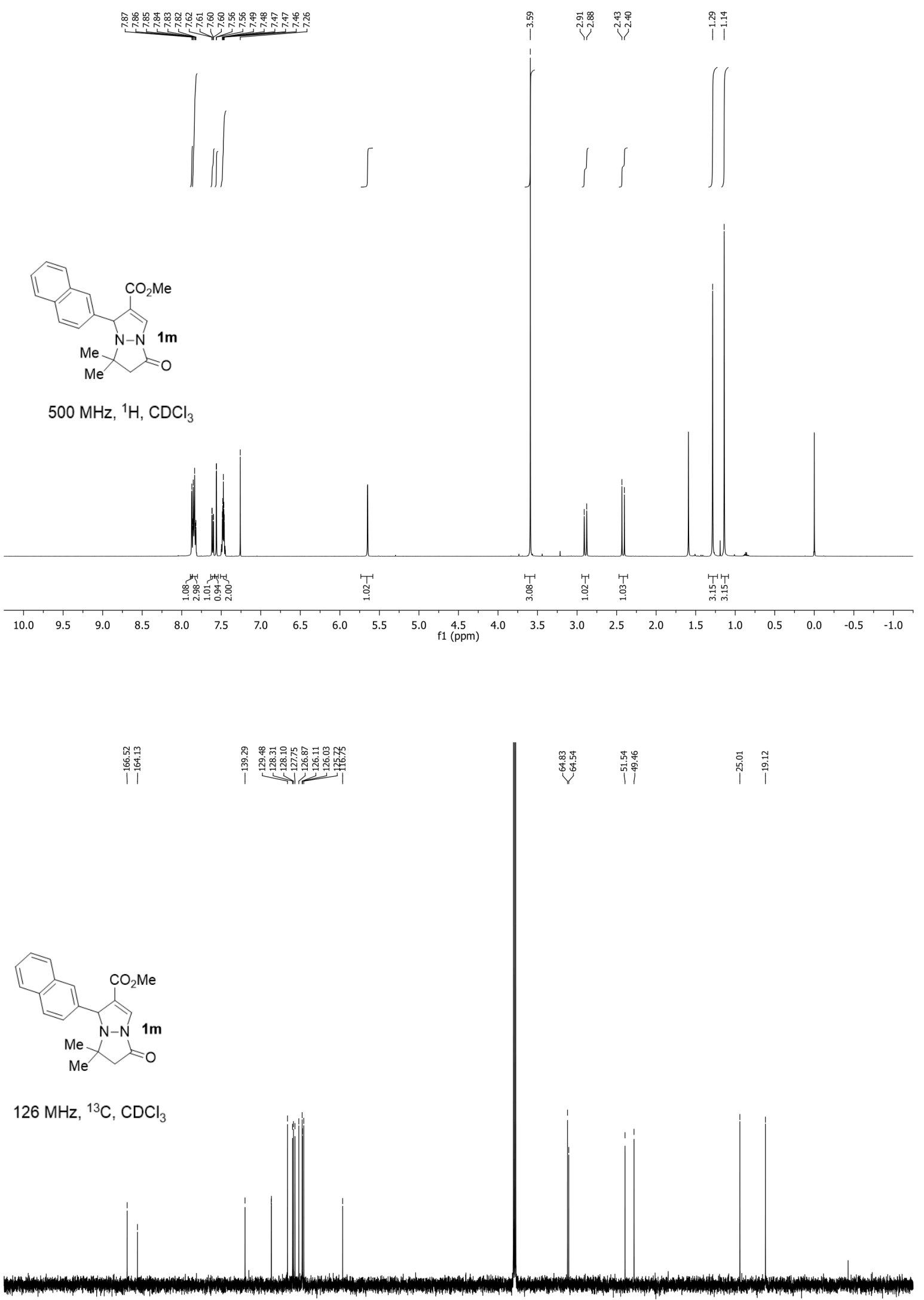

$\begin{array}{lllllllllllllllllllllll}190 & 180 & 170 & 160 & 150 & 140 & 130 & 120 & 110 & 100 & 90 & 80 & 70 & 60 & 50 & 40 & 30 & 20 & 10 & 0 & -10\end{array}$ 


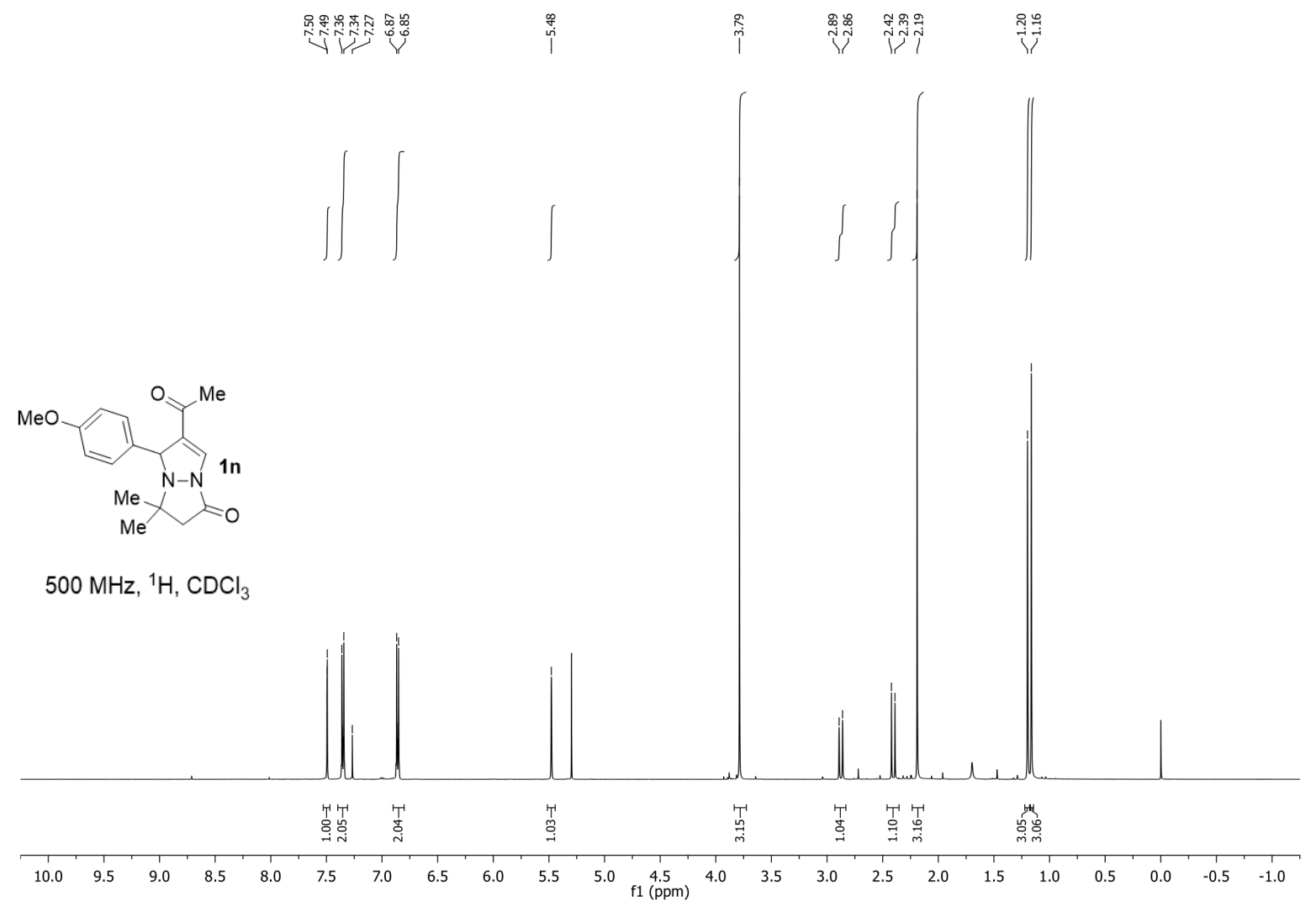

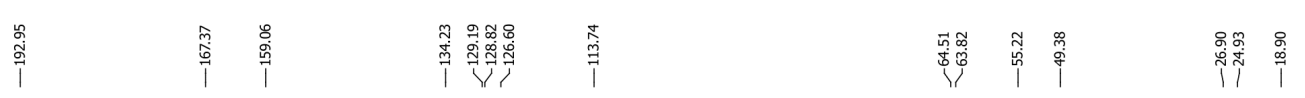

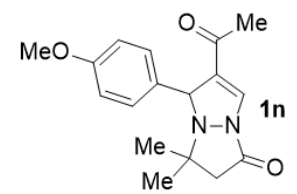

$126 \mathrm{MHz},{ }^{13} \mathrm{C}, \mathrm{CDCl}_{3}$

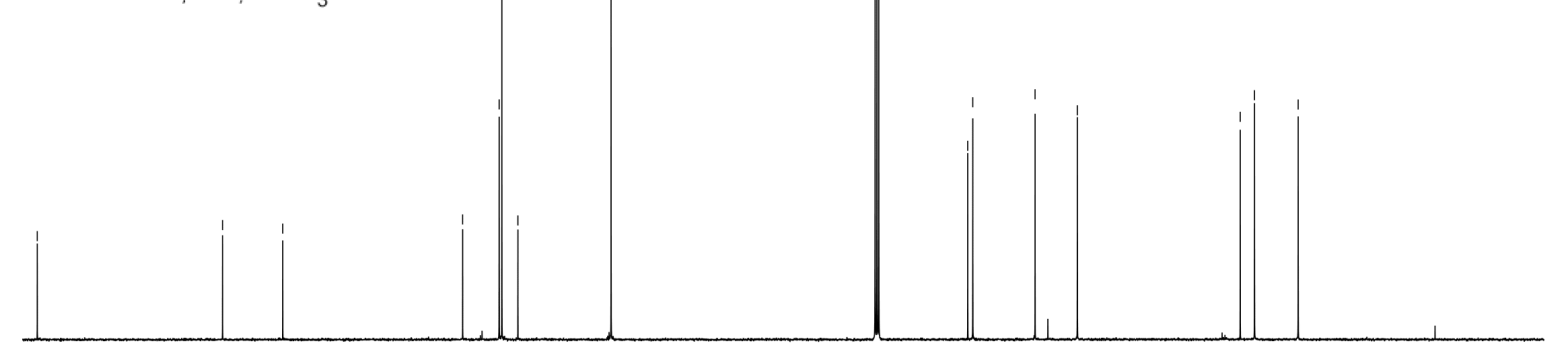

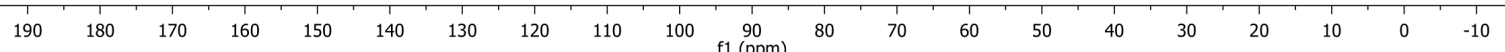



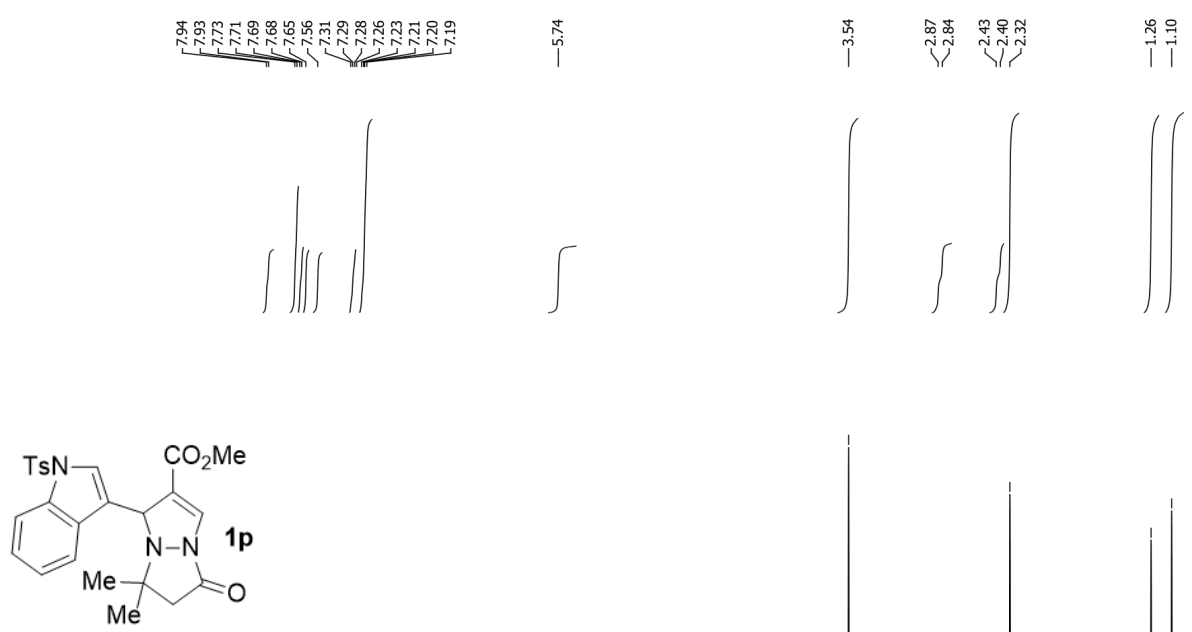

$500 \mathrm{MHz},{ }^{1} \mathrm{H}, \mathrm{CDCl}_{3}$

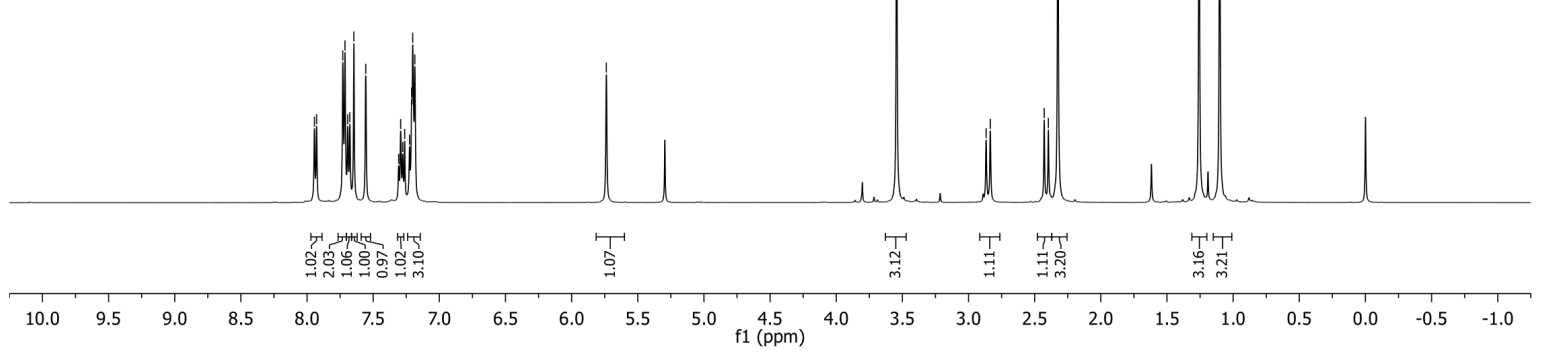
1
70
000
000
11

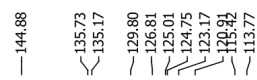

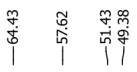

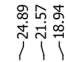

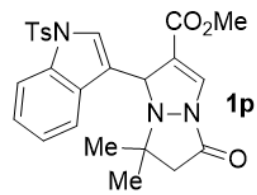

$126 \mathrm{MHz},{ }^{13} \mathrm{C}, \mathrm{CDCl}_{3}$
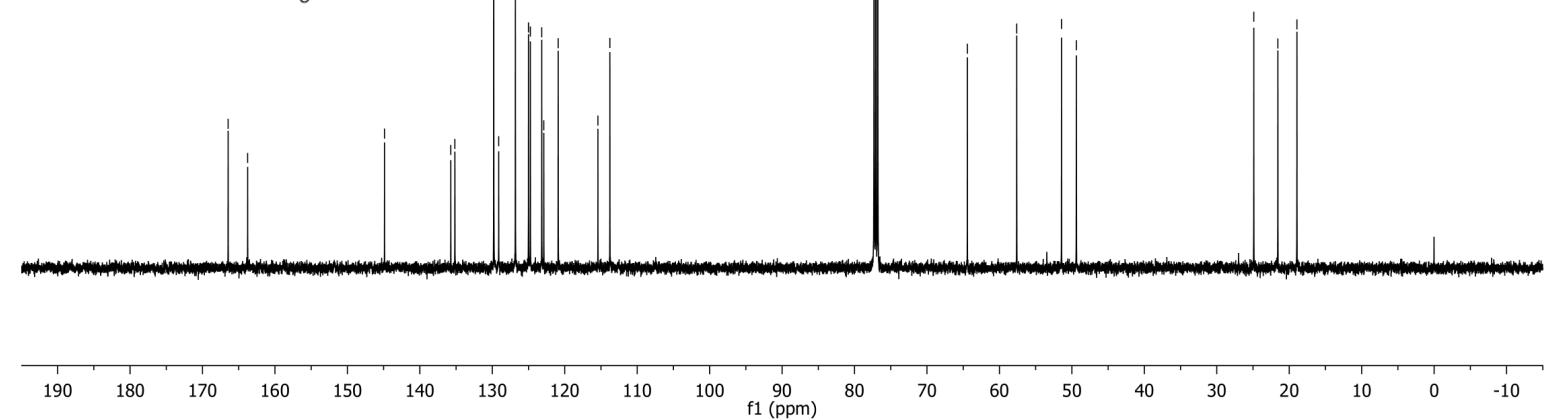

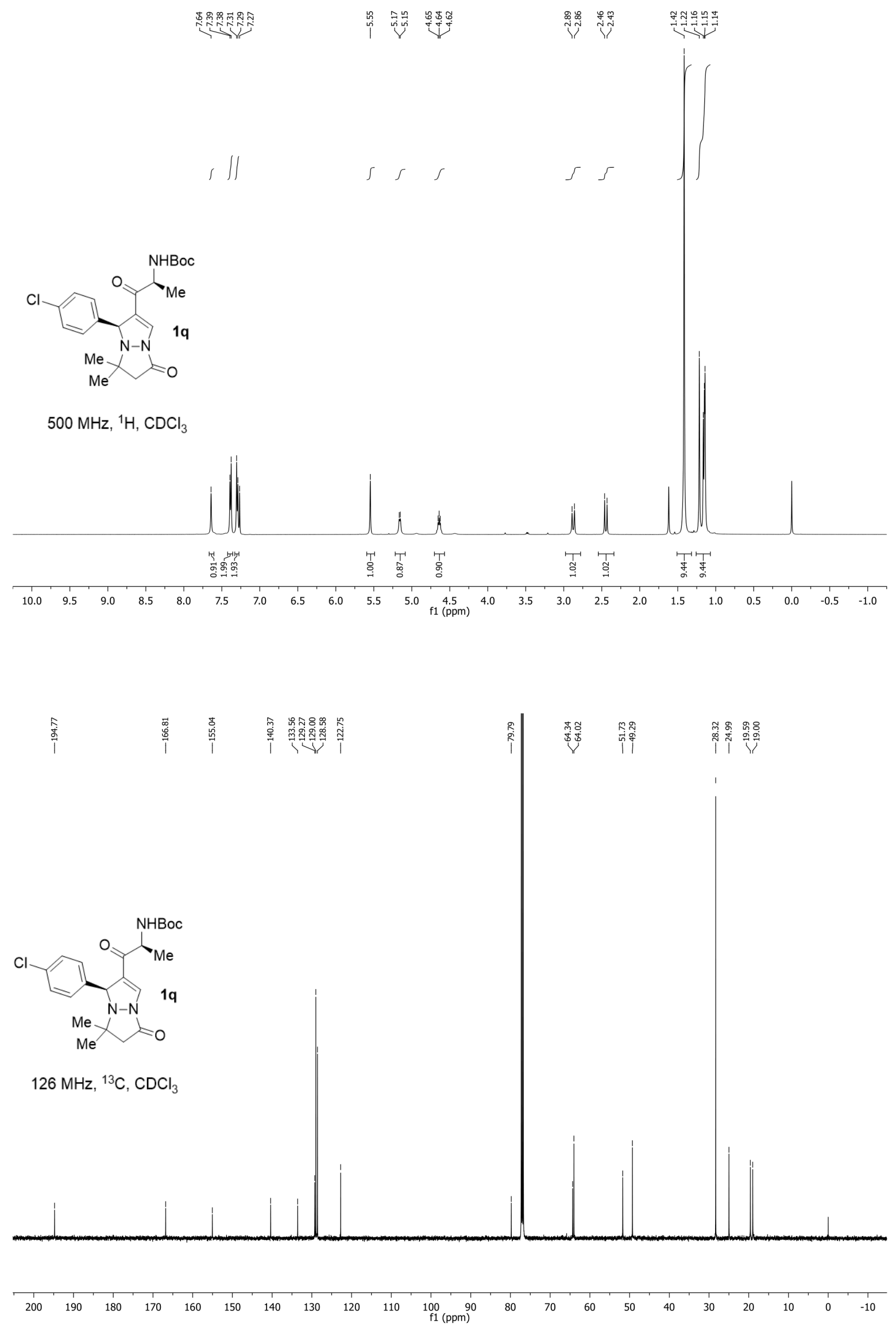

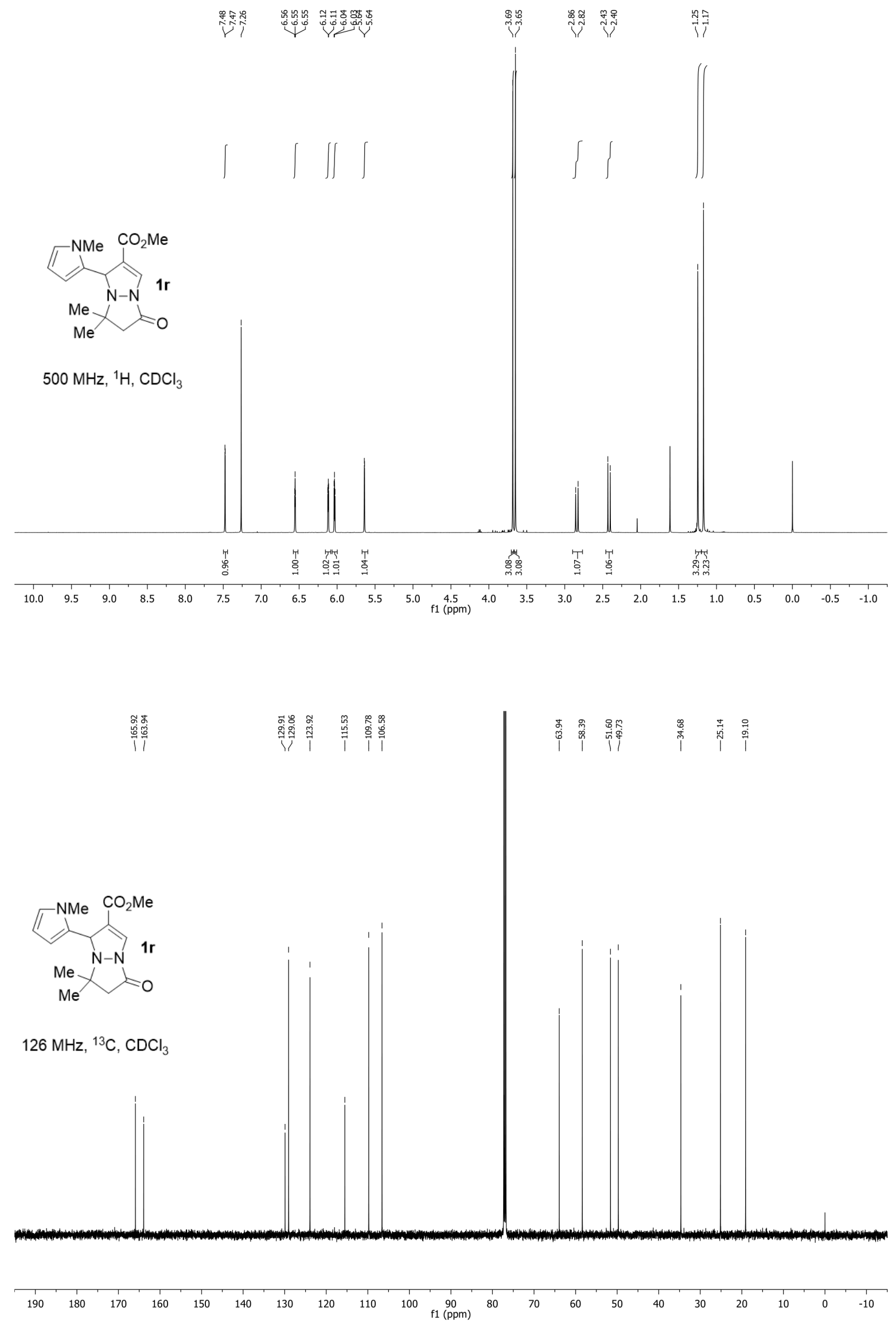

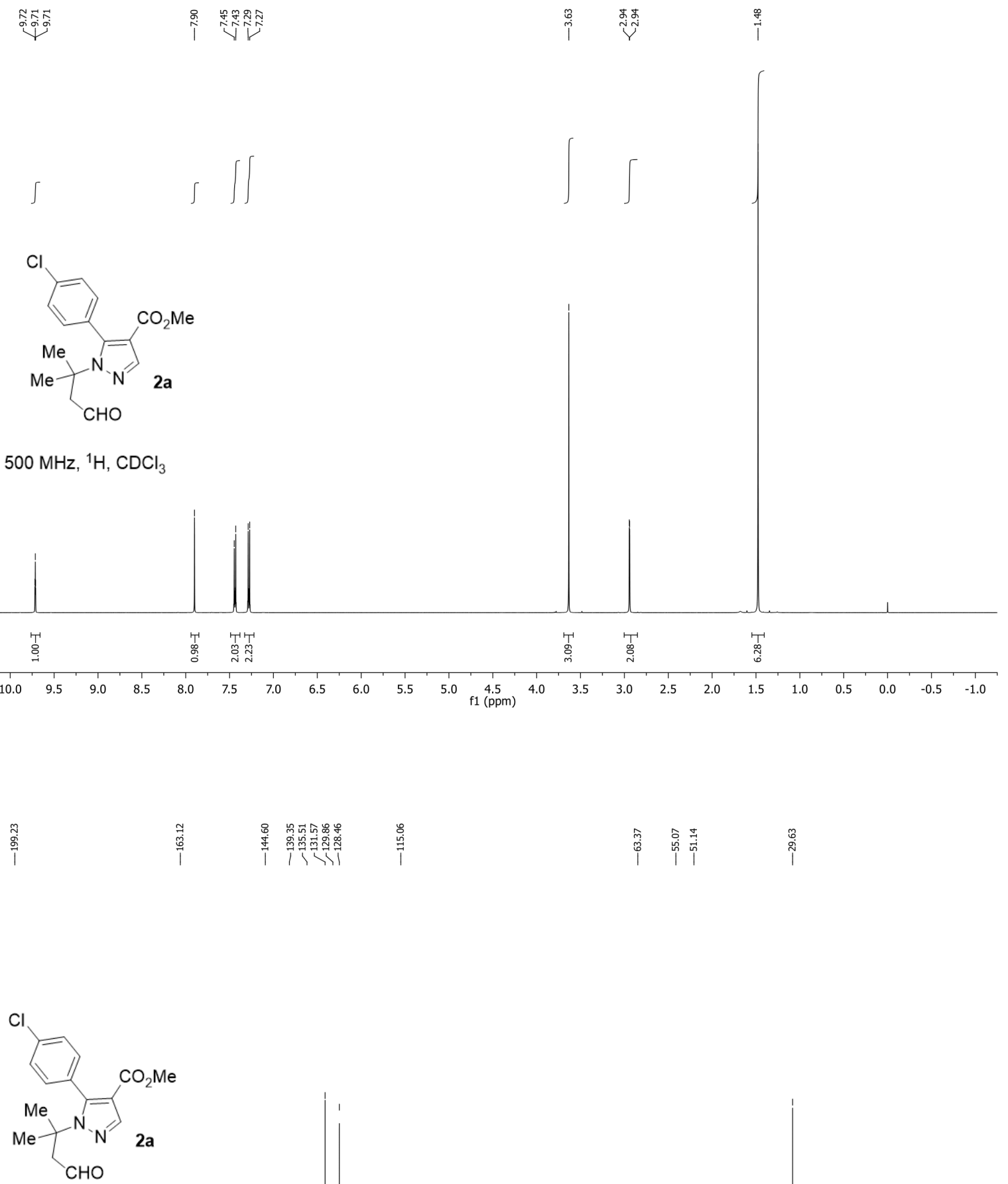

$126 \mathrm{MHz},{ }^{13} \mathrm{C}, \mathrm{CDCl}_{3}$

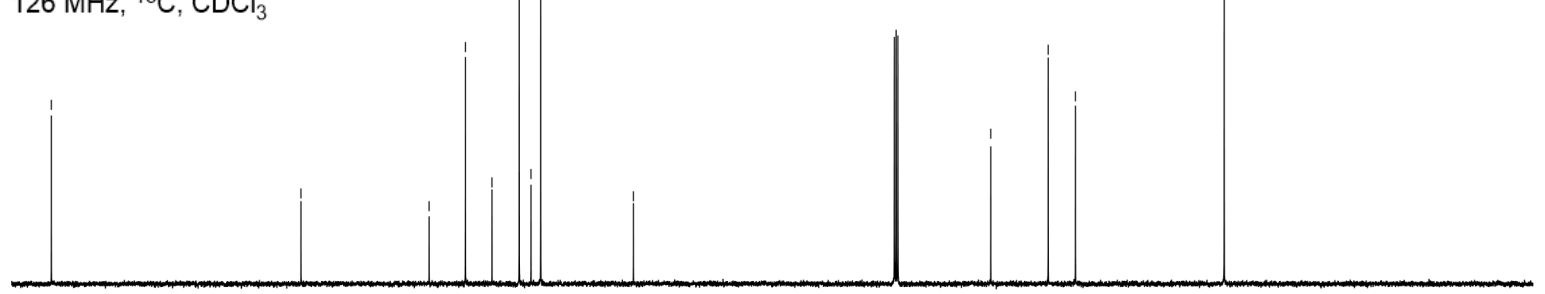

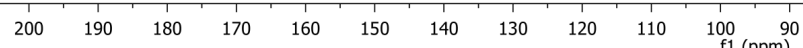



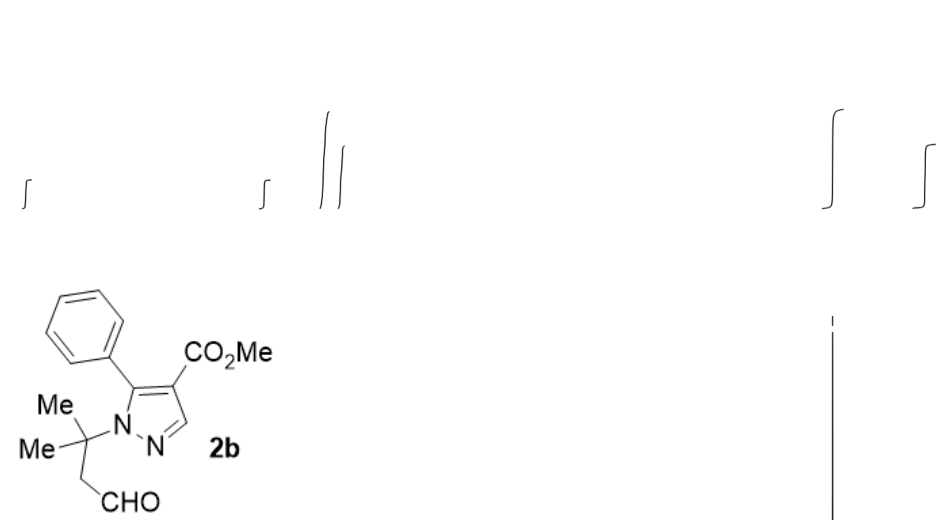

$500 \mathrm{MHz},{ }^{1} \mathrm{H}, \mathrm{CDCl}_{3}$
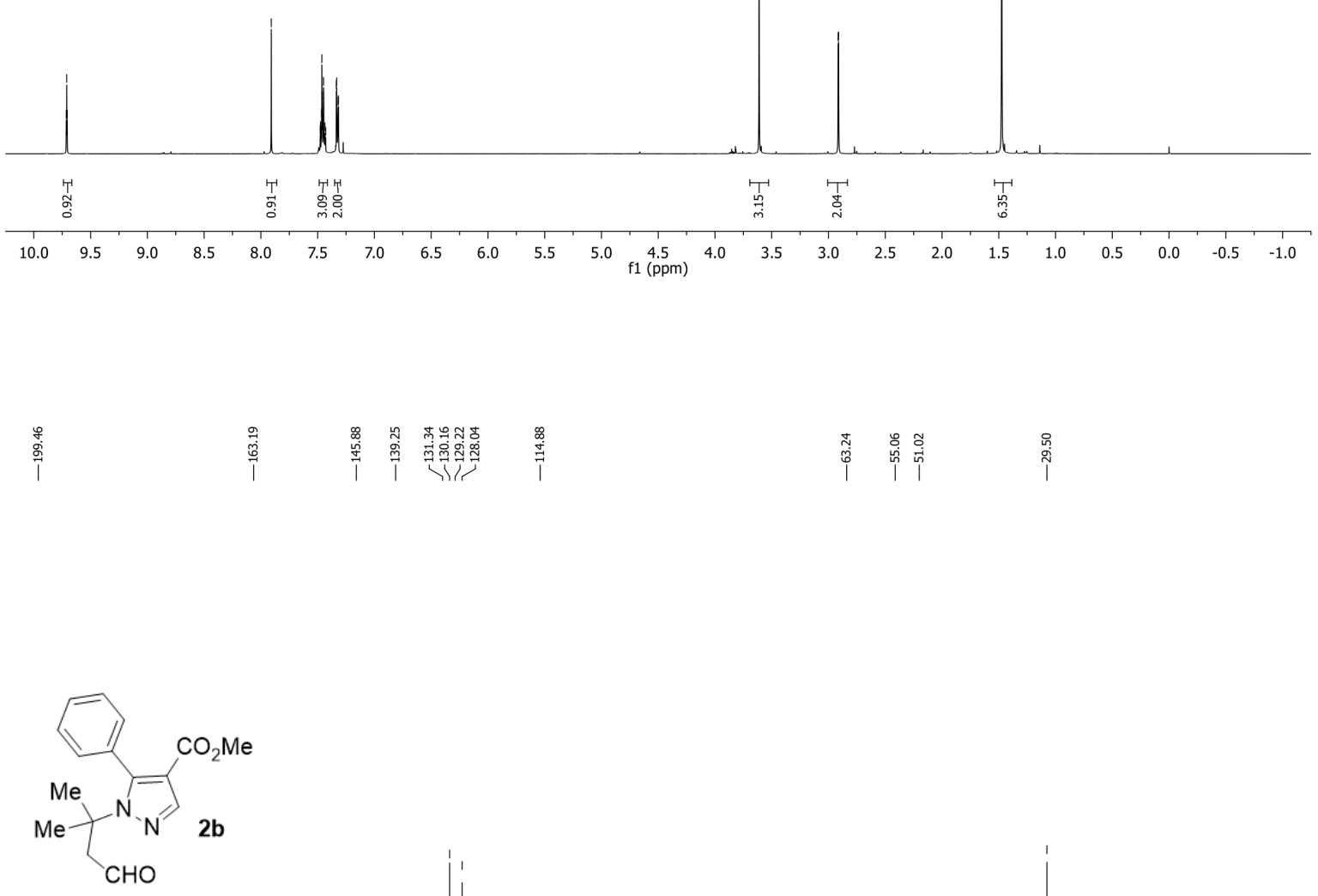

$126 \mathrm{MHz},{ }^{13} \mathrm{C}, \mathrm{CDCl}_{3}$

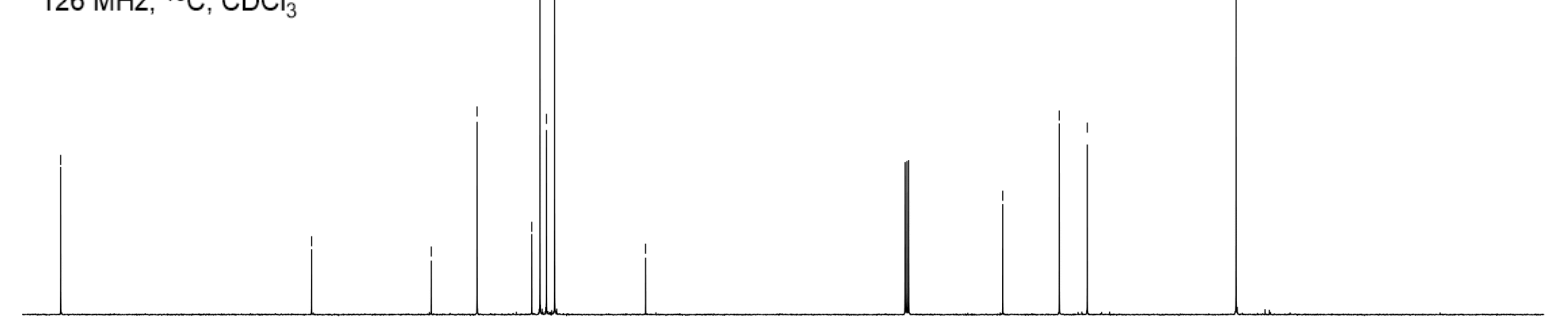




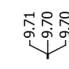

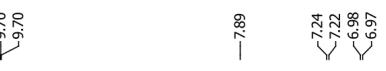

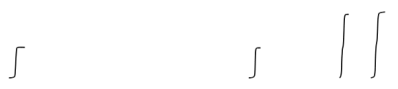

$\mathrm{MeO}$

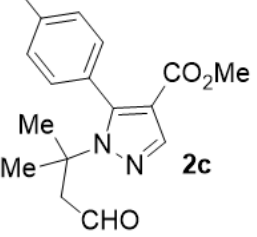

$500 \mathrm{MHz},{ }^{1} \mathrm{H}, \mathrm{CDCl}_{3}$

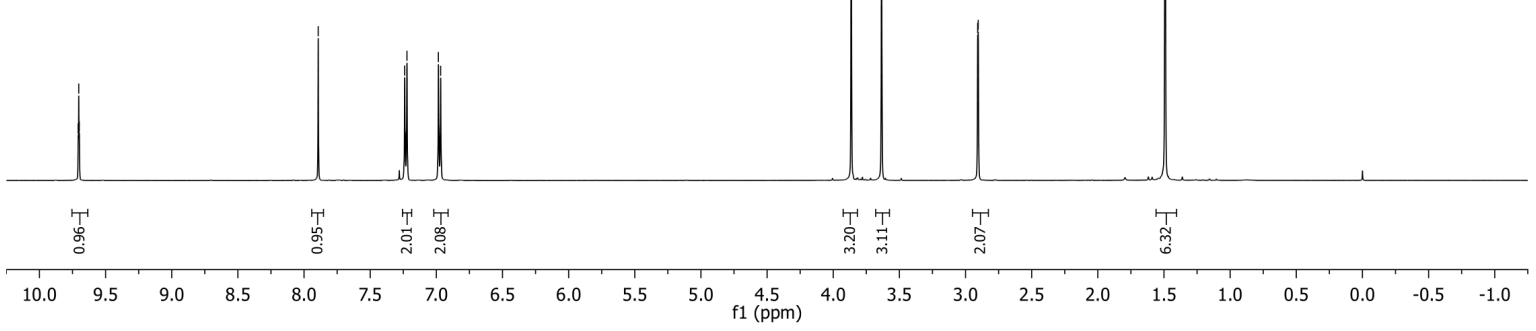

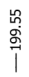

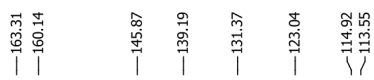

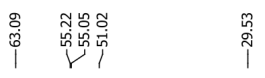

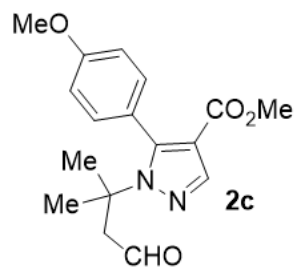

$126 \mathrm{MHz},{ }^{13} \mathrm{C}, \mathrm{CDCl}_{3}$

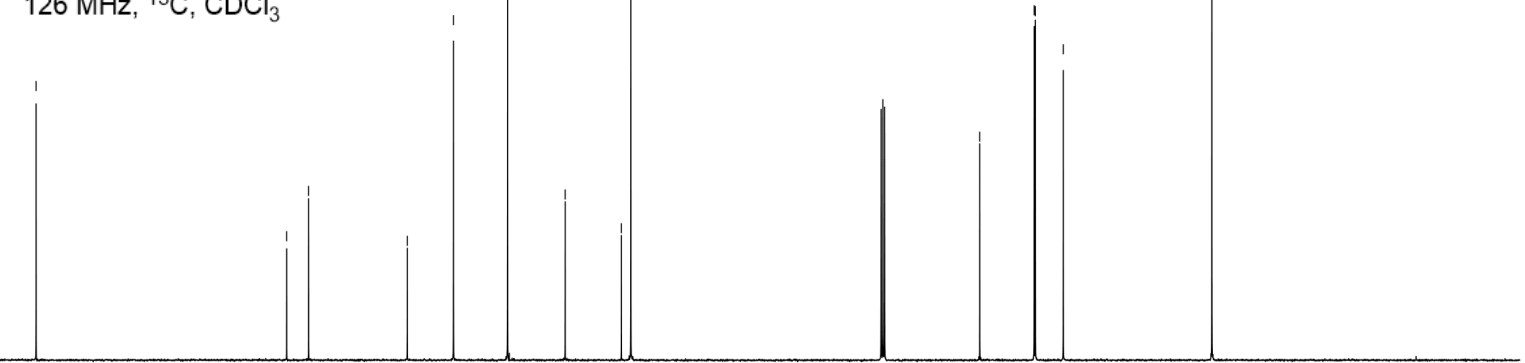

200

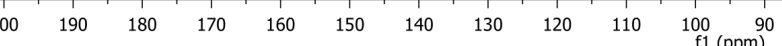



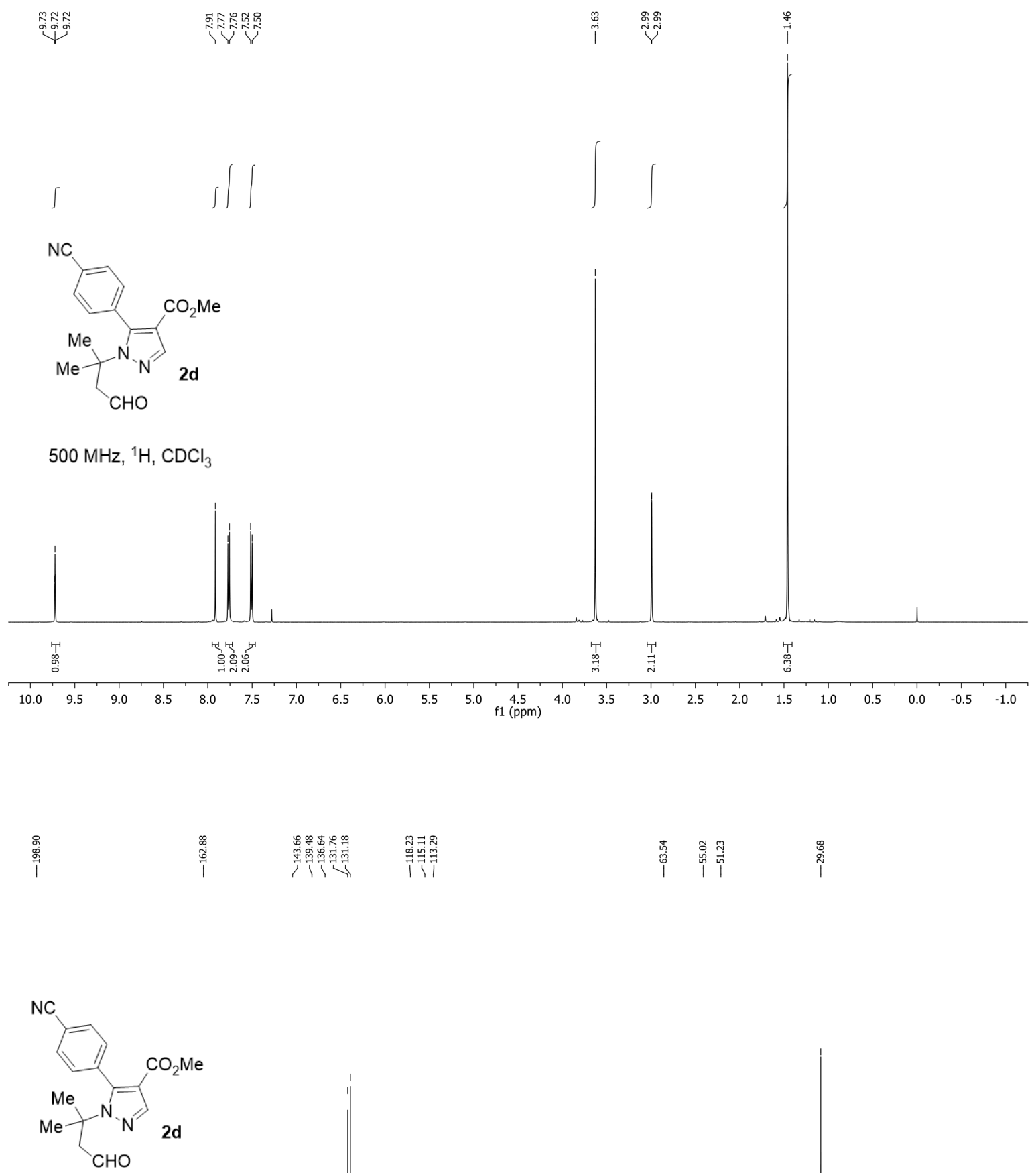

$126 \mathrm{MHz},{ }^{13} \mathrm{C}, \mathrm{CDCl}_{3}$

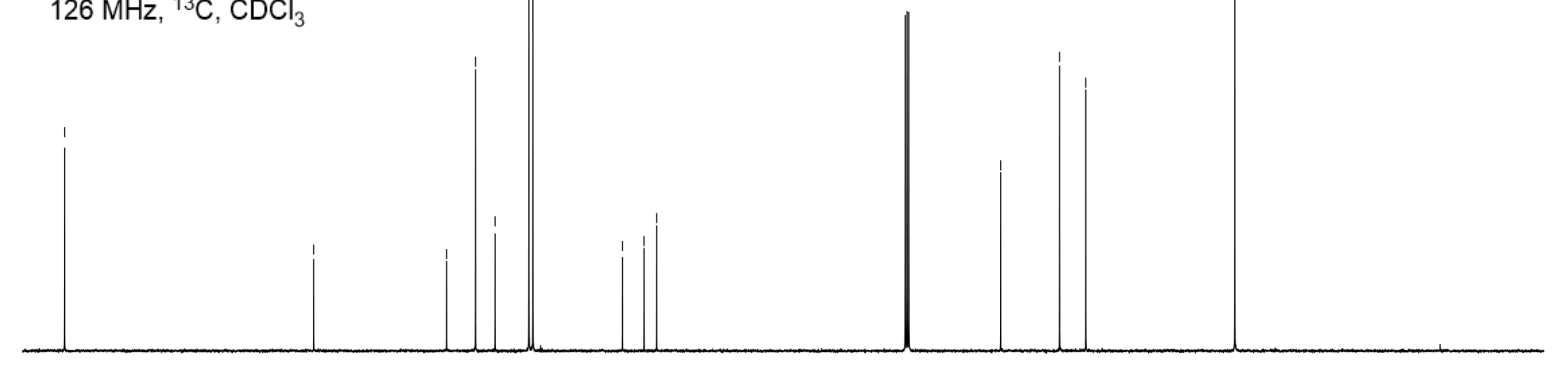

$\begin{array}{lllllllllllllllllllllllllllll}200 & 190 & 180 & 170 & 160 & 150 & 140 & 130 & 120 & 110 & 100 & 90 & 80 & 70 & 60 & 50 & 40 & 30 & 20 & 10 & 0 & -10\end{array}$ 


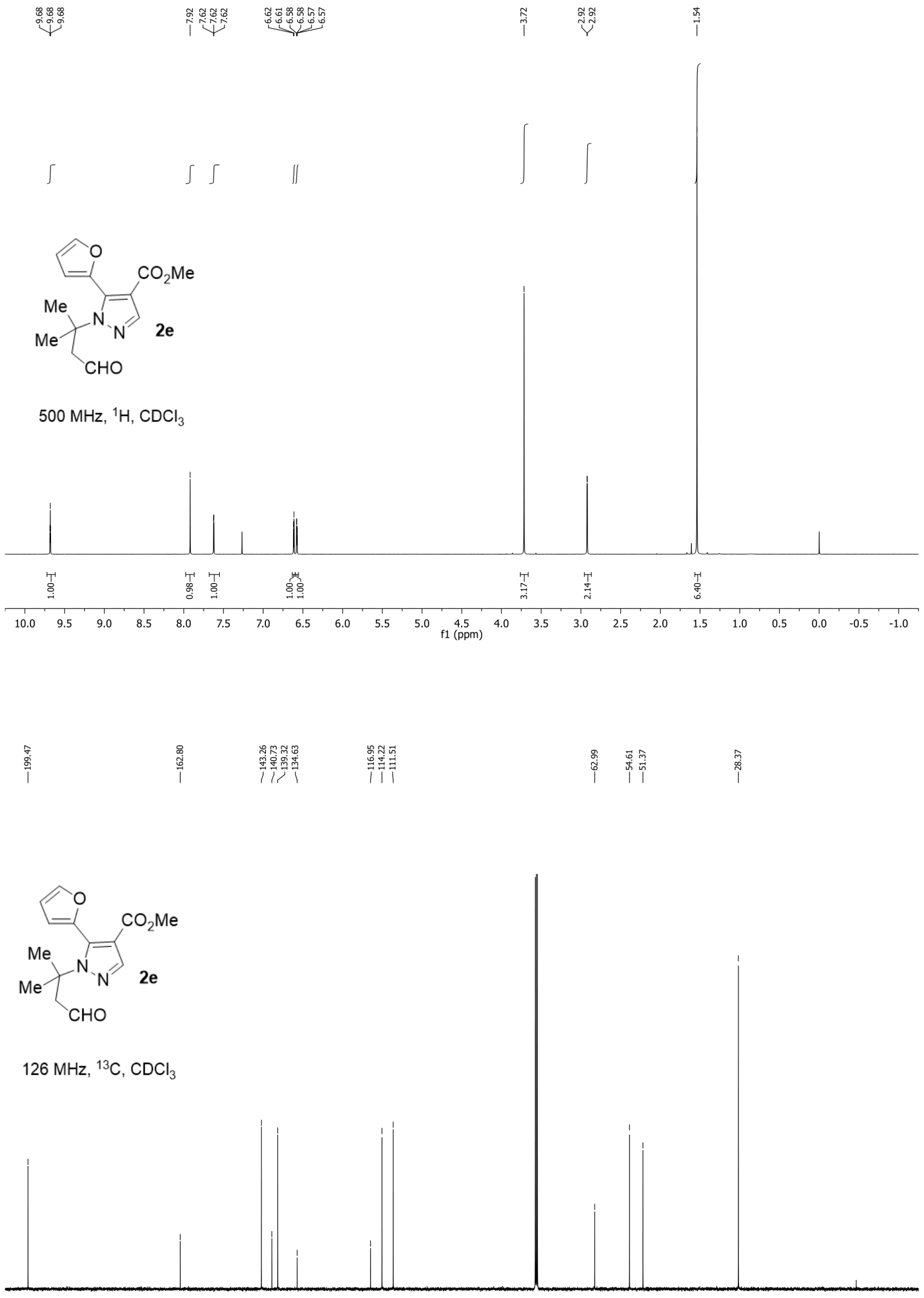

$\begin{array}{llllllllllllllllllllllllllll}200 & 190 & 180 & 170 & 160 & 150 & 140 & 130 & 120 & 110 & 100 & 90 & 80 & 70 & 60 & 50 & 40 & 30 & 20 & 10 & 0 & -10\end{array}$ 

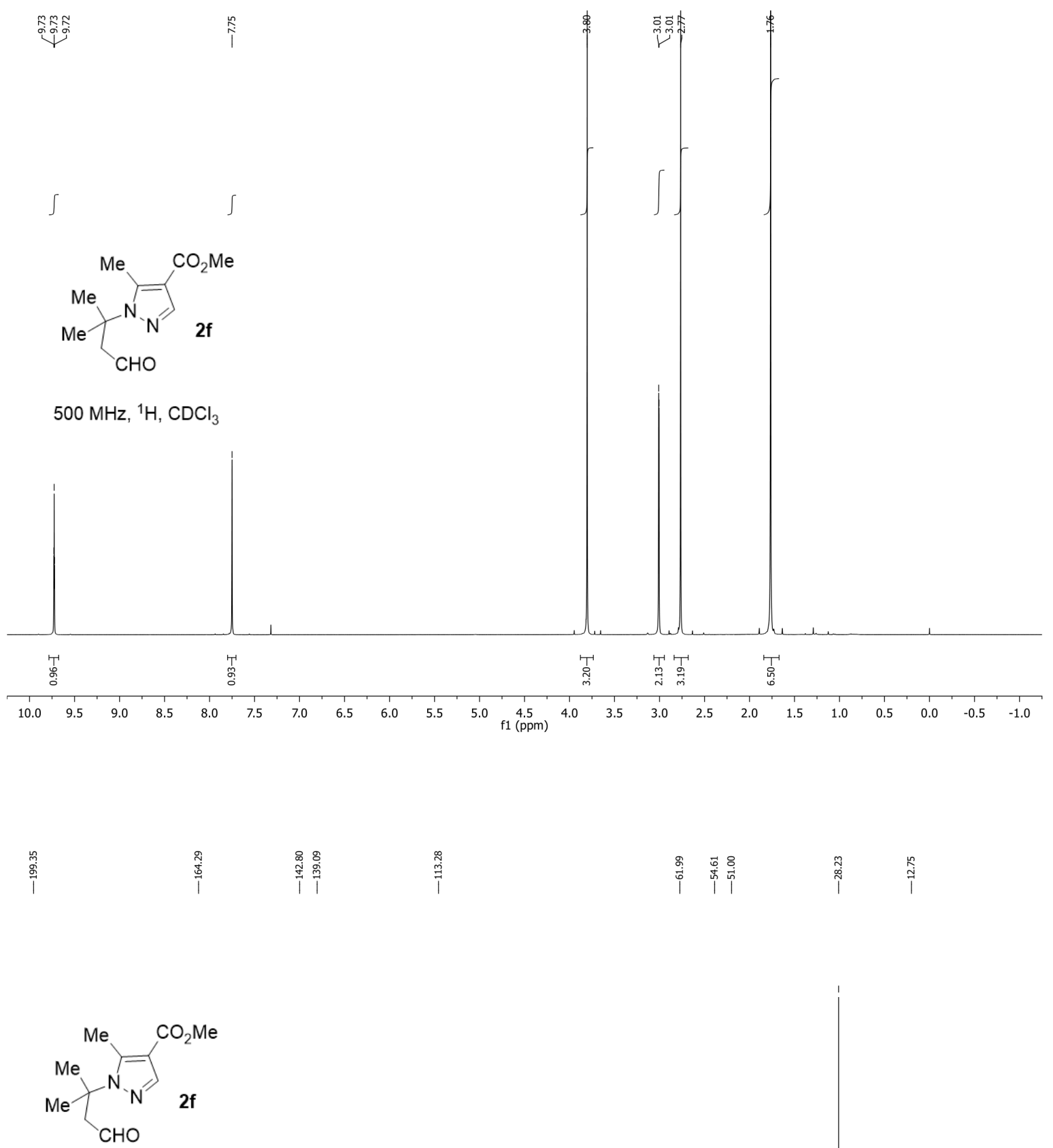

$126 \mathrm{MHz},{ }^{13} \mathrm{C}, \mathrm{CDCl}_{3}$

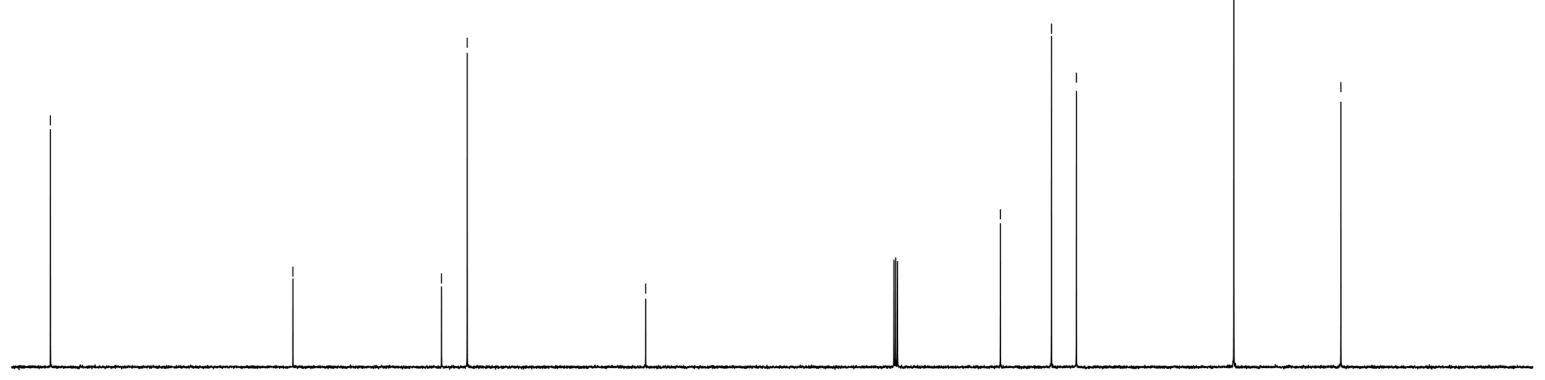

$\begin{array}{llllllllllllllllllllllllllll}200 & 190 & 180 & 170 & 160 & 150 & 140 & 130 & 120 & 110 & 100 & 90 & 80 & 70 & 60 & 50 & 40 & 30 & 20 & 10 & 0 & -10\end{array}$ 

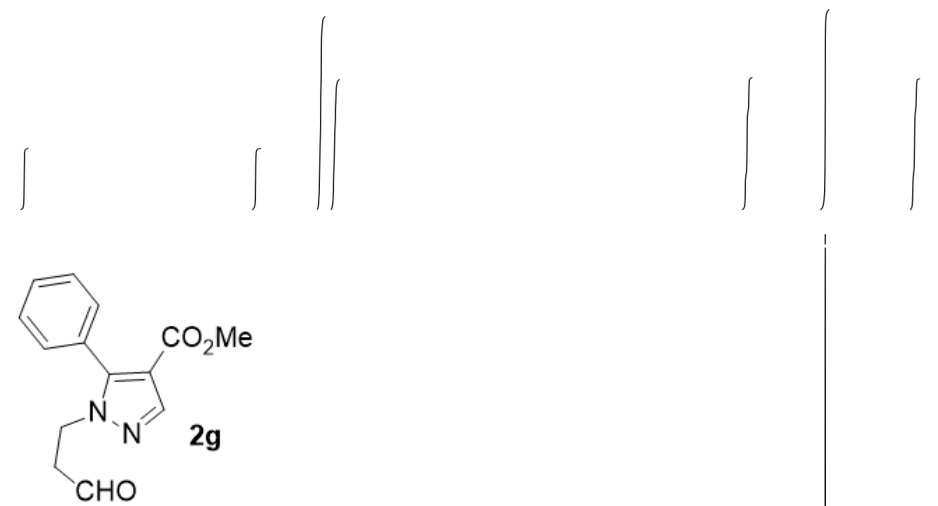

$500 \mathrm{MHz},{ }^{1} \mathrm{H}, \mathrm{CDCl}_{3}$
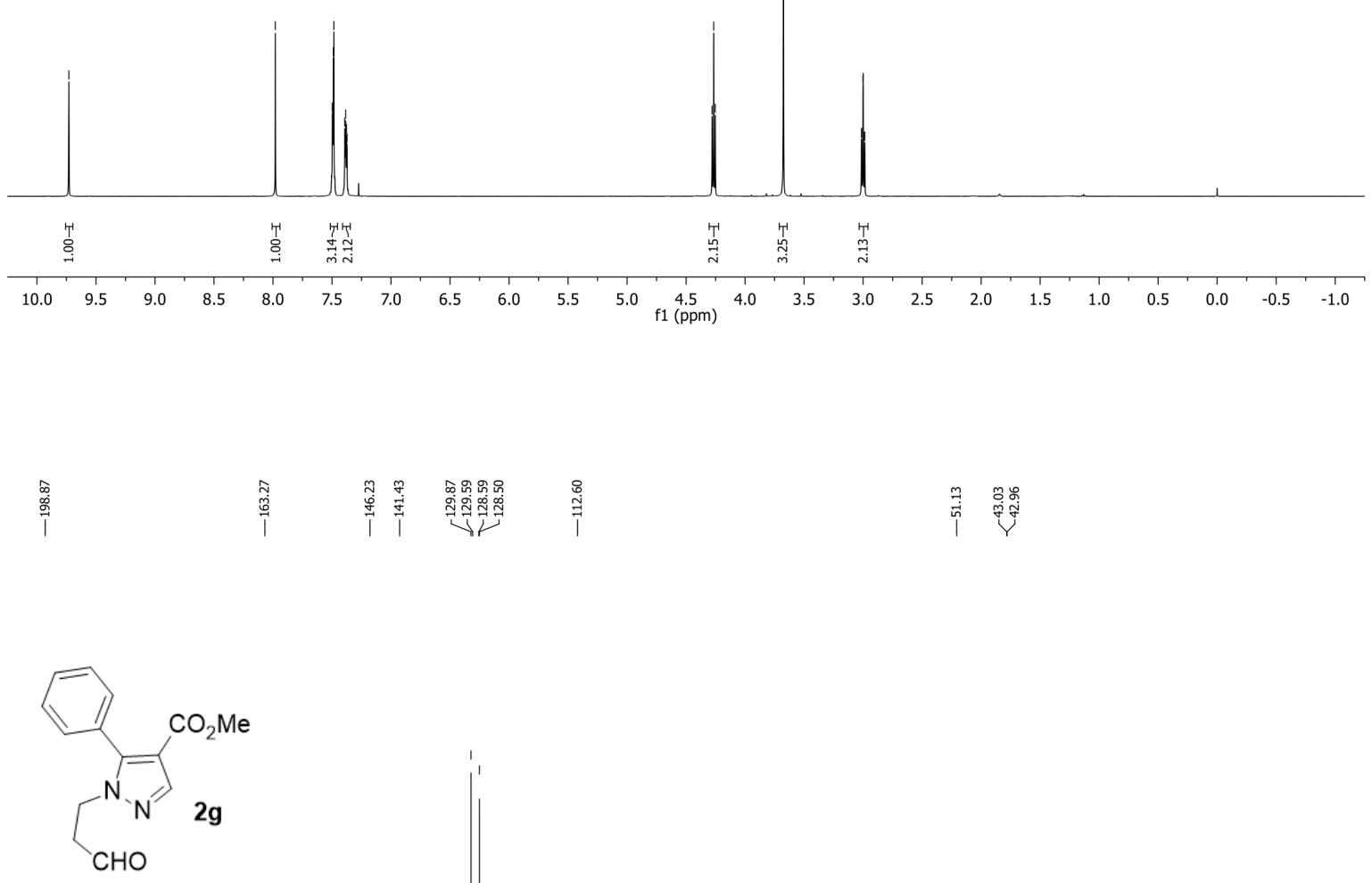

$126 \mathrm{MHz},{ }^{13} \mathrm{C}, \mathrm{CDCl}_{3}$

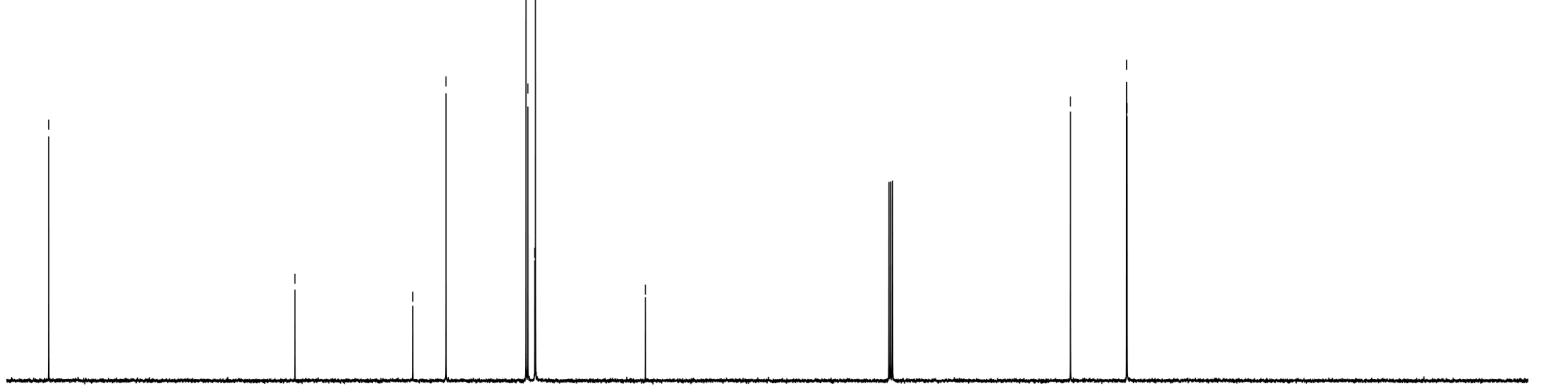

$\begin{array}{lllllllllllllllllllllll} & 1 \\ 200 & 190 & 180 & 170 & 160 & 150 & 140 & 130 & 120 & 110 & 100 & 90 & 80 & 70 & 60 & 50 & 40 & 30 & 20 & 10 & 0 & -10\end{array}$ 

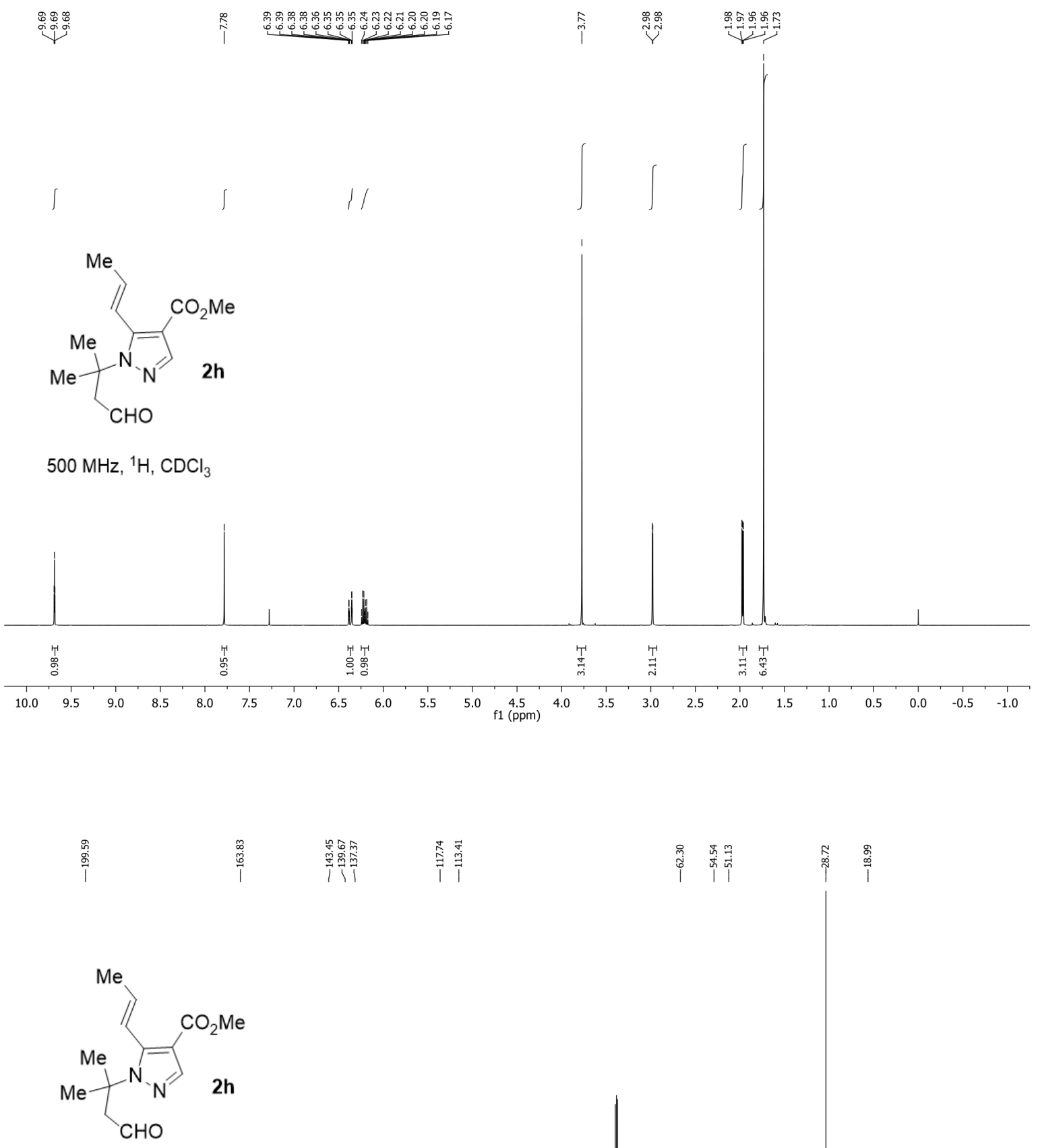

$126 \mathrm{MHz},{ }^{13} \mathrm{C}, \mathrm{CDCl}_{3}$

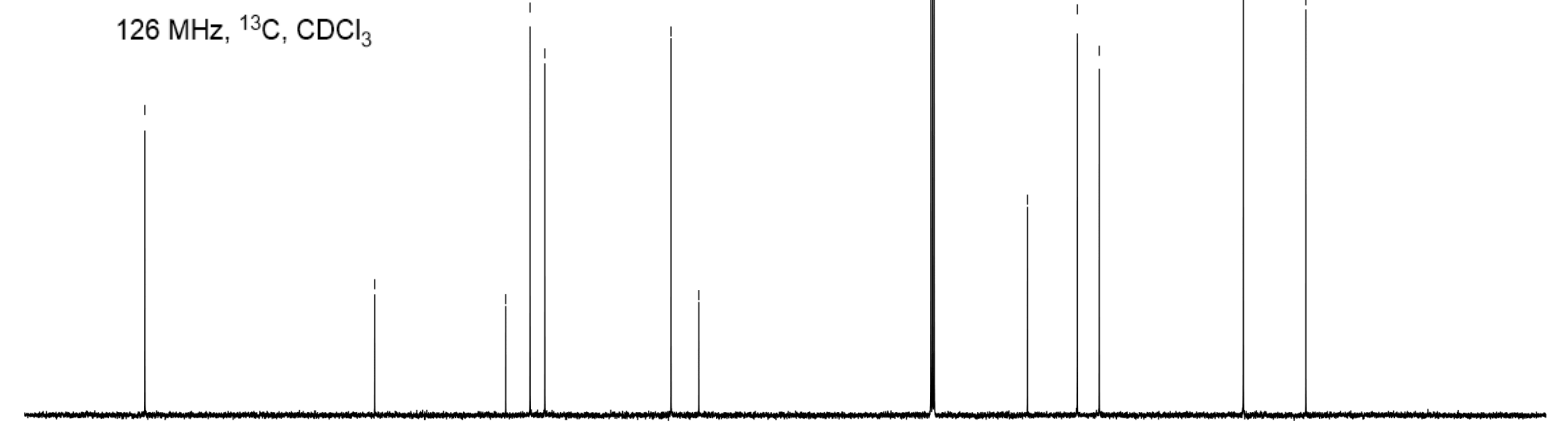

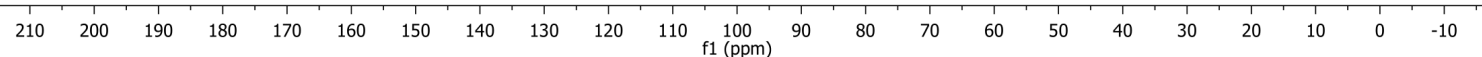



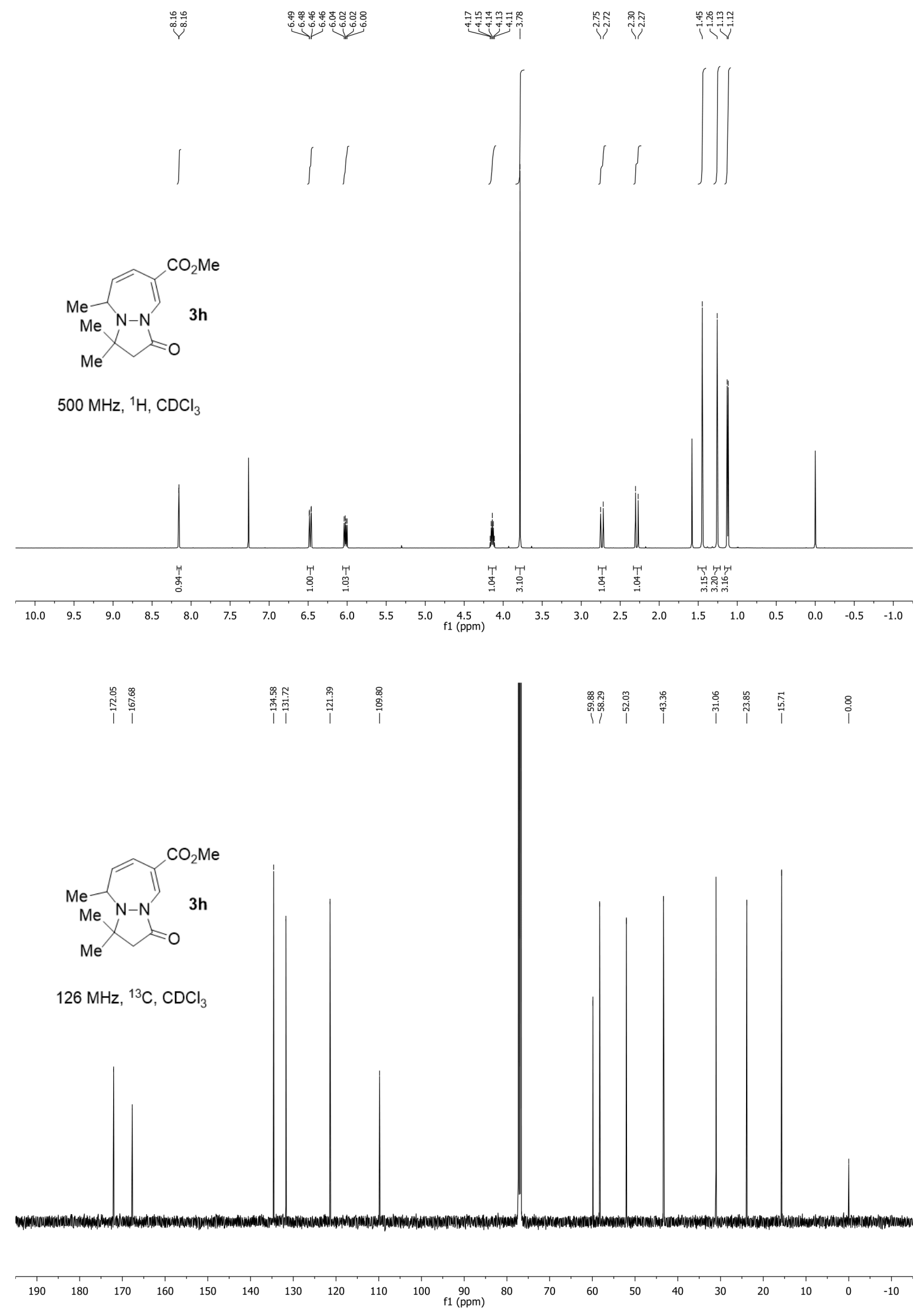


\section{พุ๊}

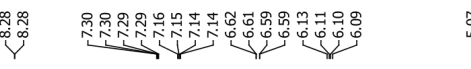

盖品亭

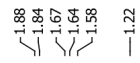
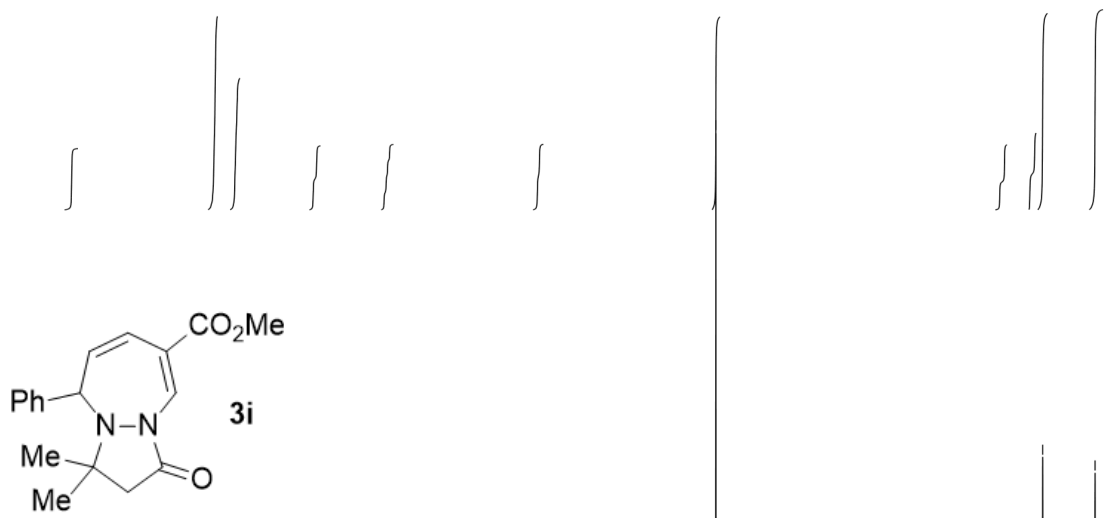

$500 \mathrm{MHz},{ }^{1} \mathrm{H}, \mathrm{CDCl}_{3}$

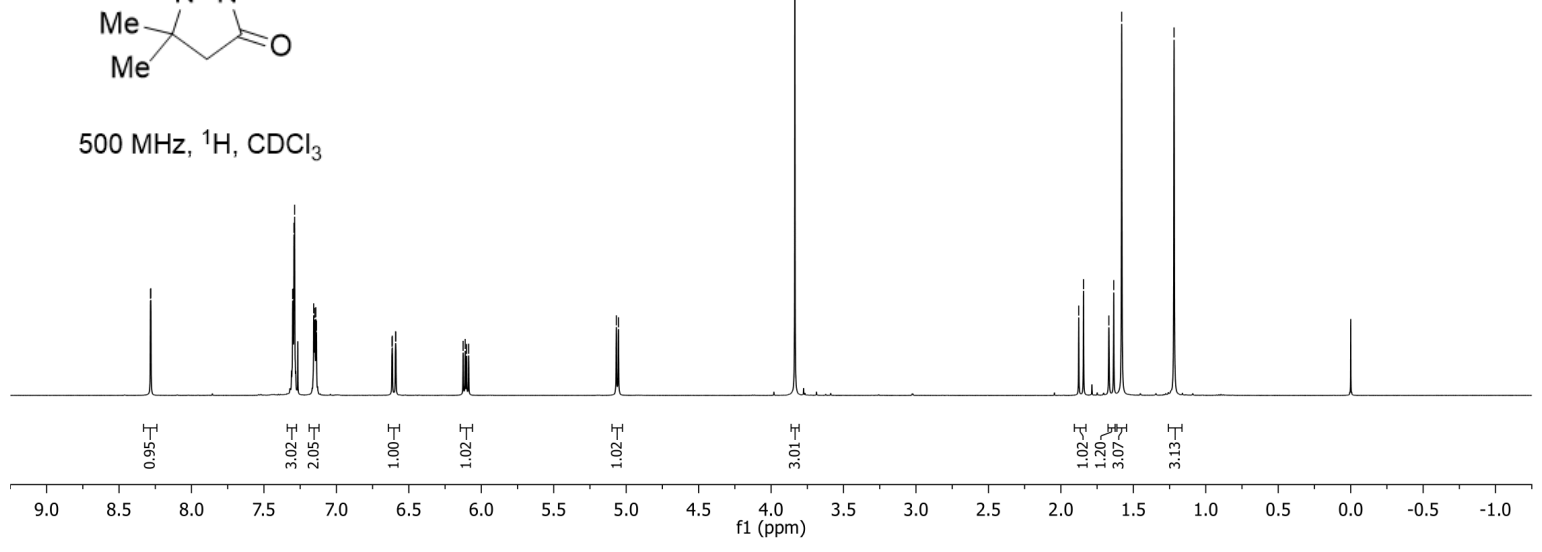

i

|

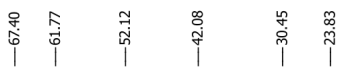

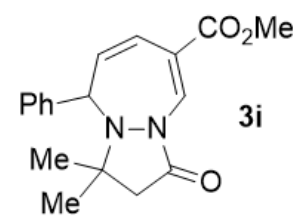

$126 \mathrm{MHz},{ }^{13} \mathrm{C}, \mathrm{CDCl}_{3}$

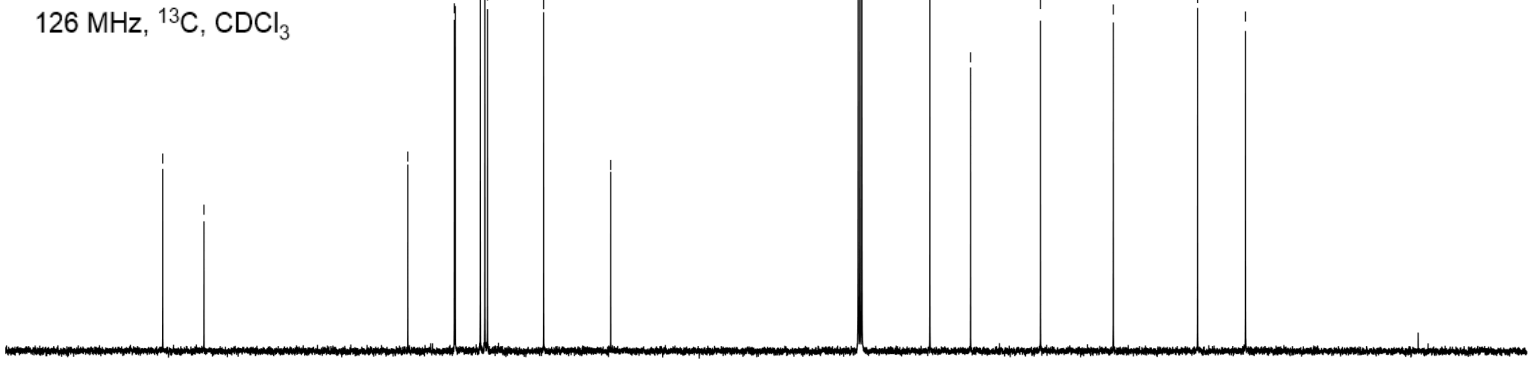




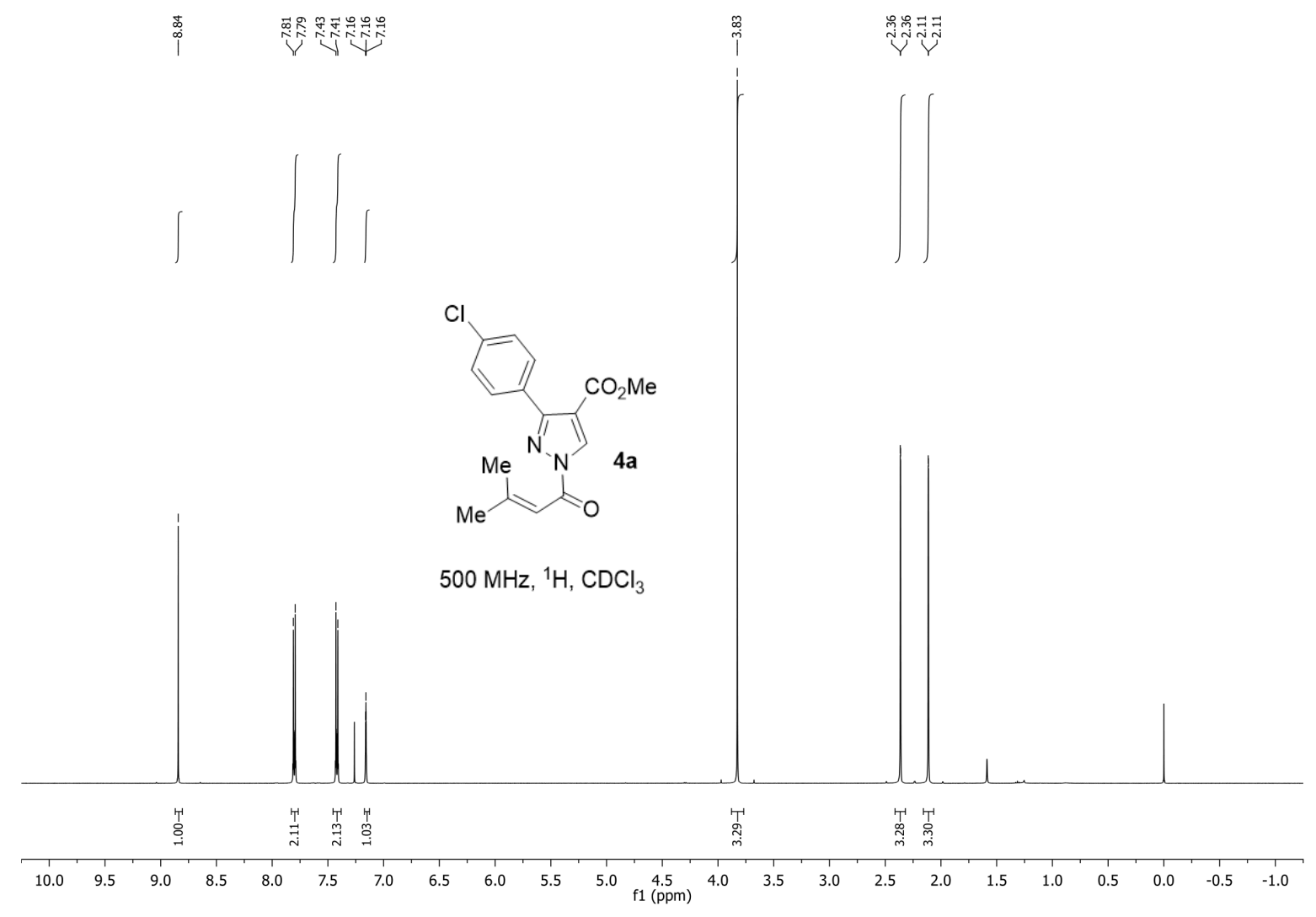

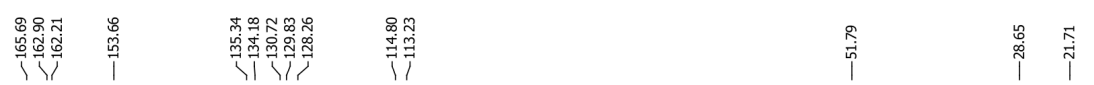

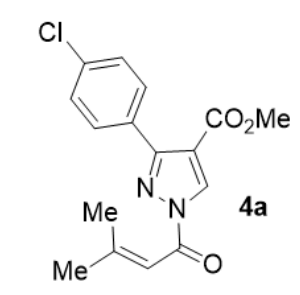

$126 \mathrm{MHz},{ }^{13} \mathrm{C}, \mathrm{CDCl}_{3}$

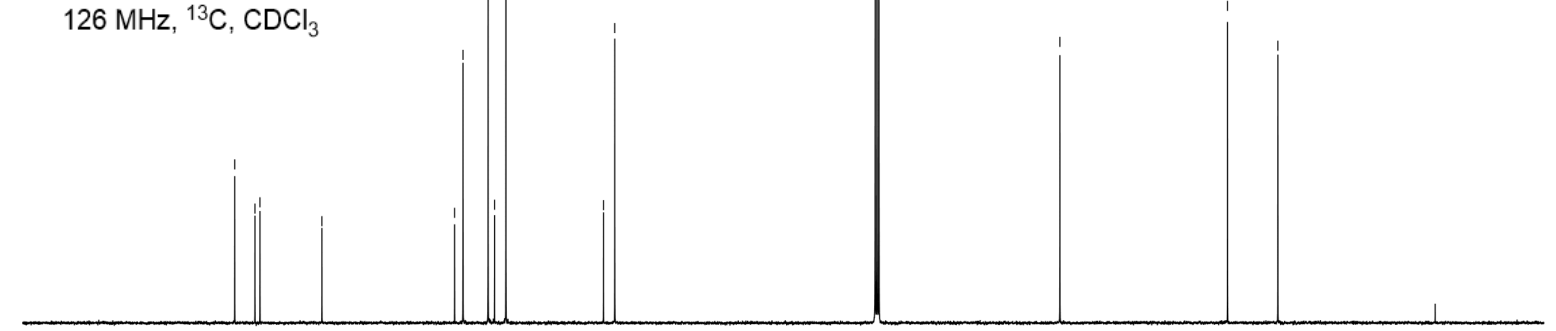

$\begin{array}{lllllllllllllllllllllll}190 & 180 & 170 & 160 & 150 & 140 & 130 & 120 & 110 & 100 & 90 & 80 & 70 & 60 & 50 & 40 & 30 & 20 & 10 & 0 & -10\end{array}$ 


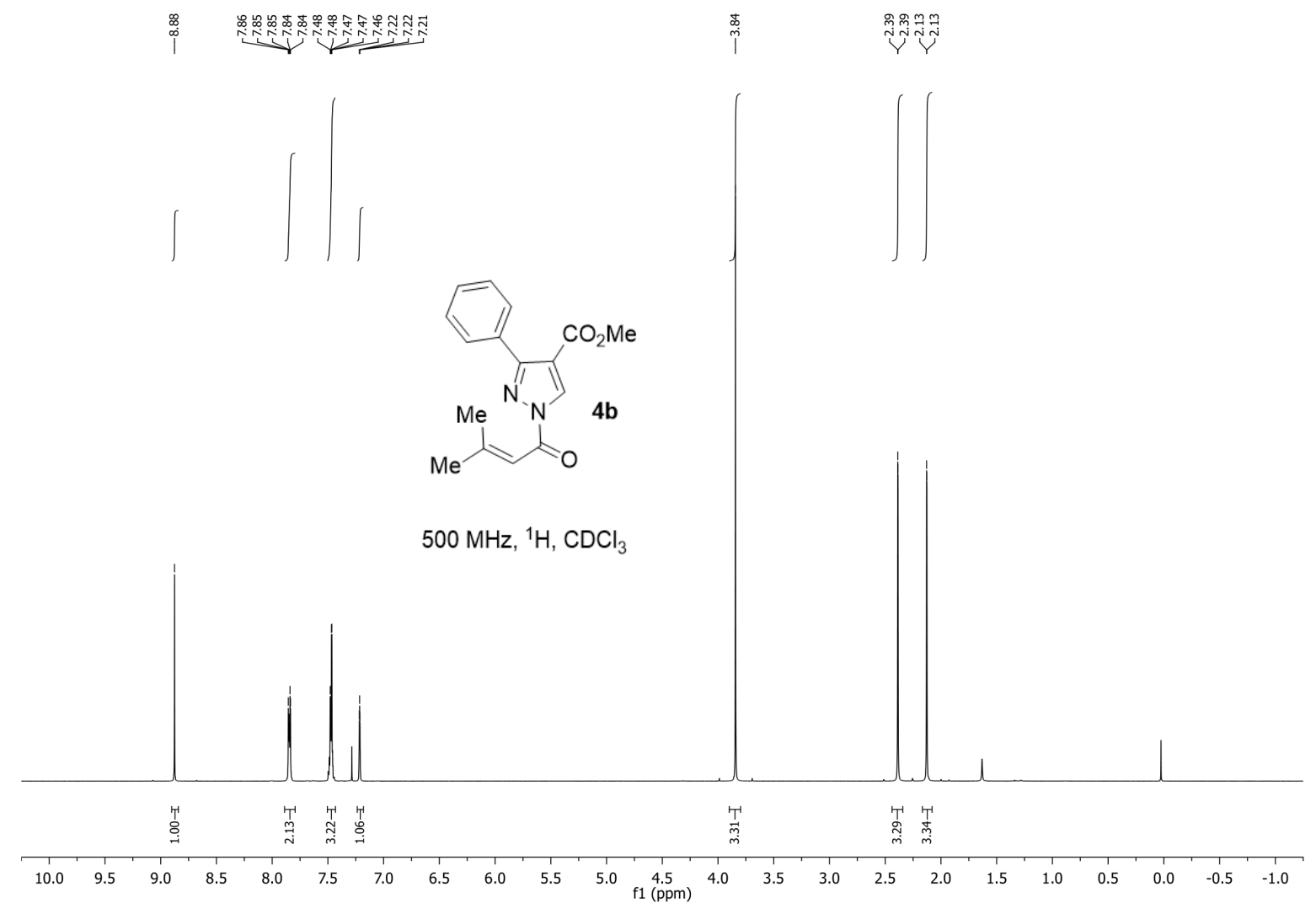

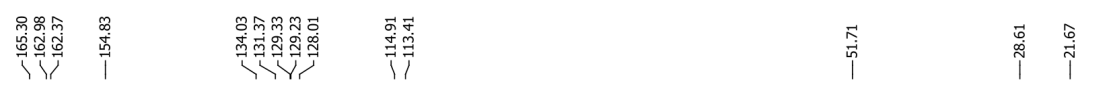

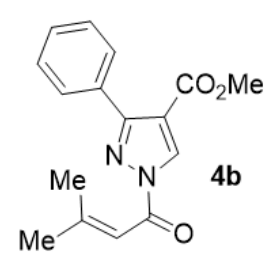

$126 \mathrm{MHz},{ }^{13} \mathrm{C}, \mathrm{CDCl}_{3}$

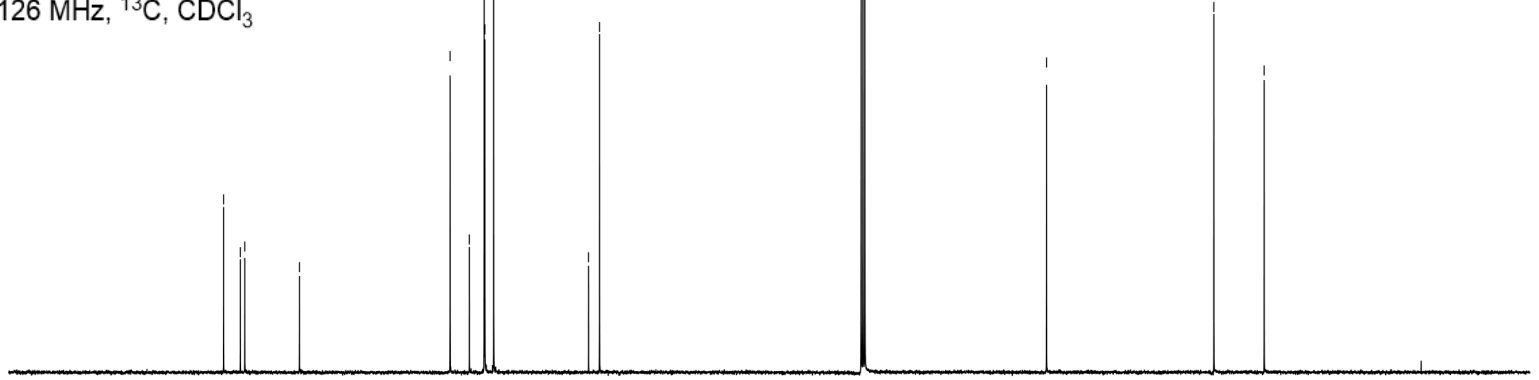

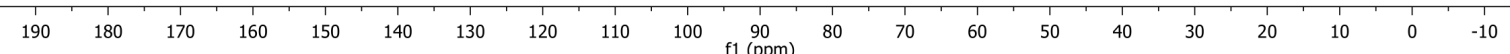



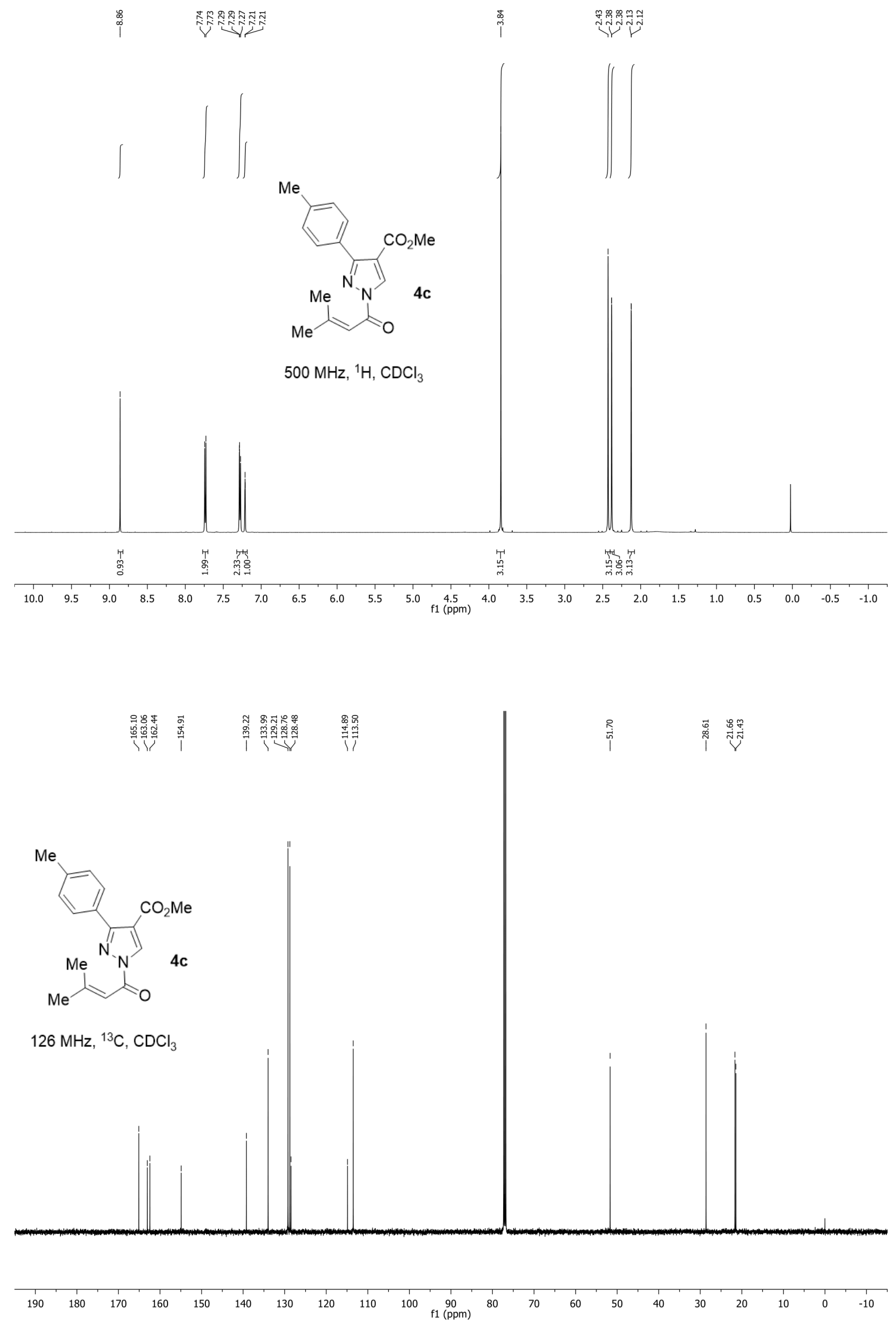

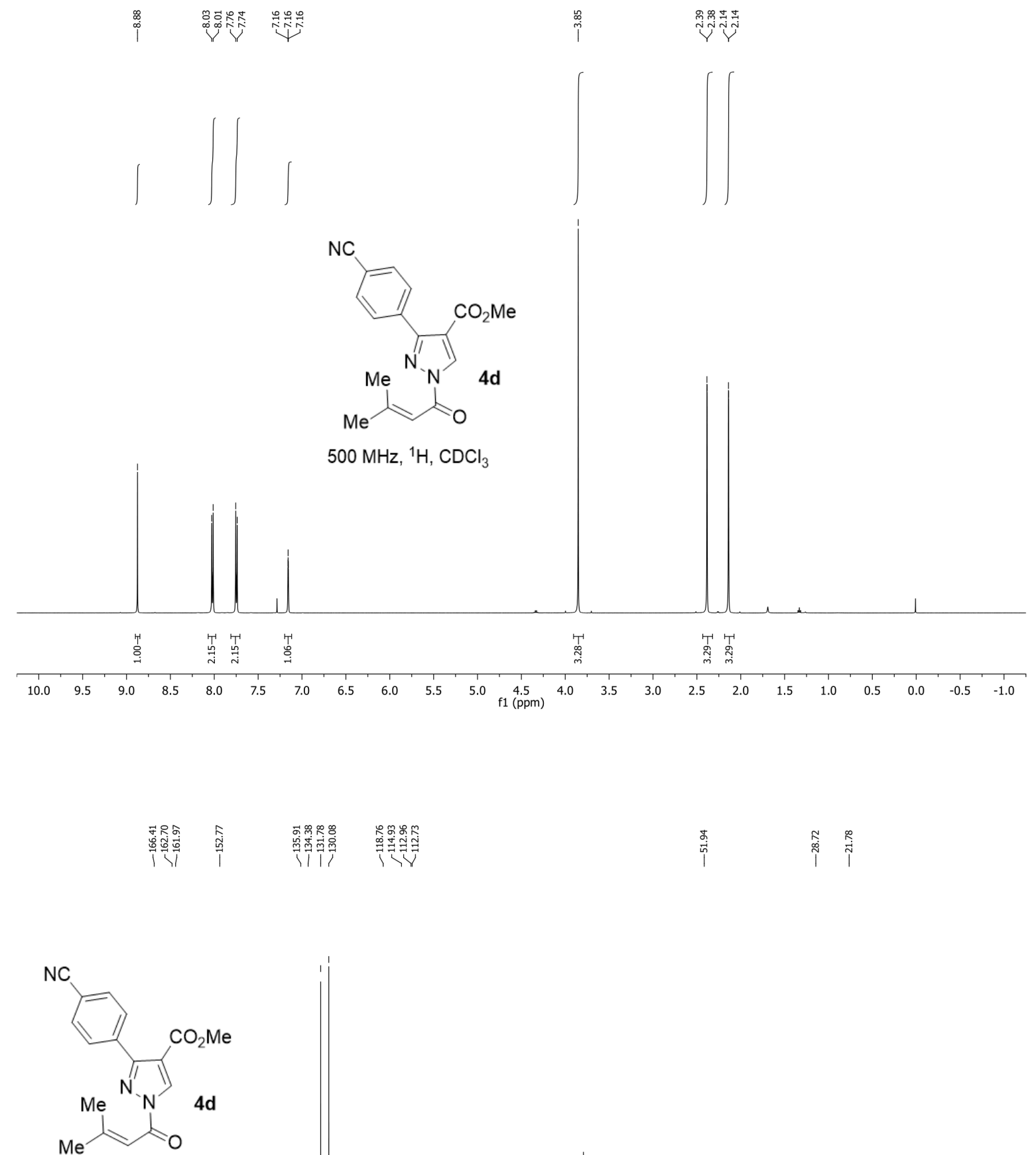

$126 \mathrm{MHz},{ }^{13} \mathrm{C}, \mathrm{CDCl}_{3}$

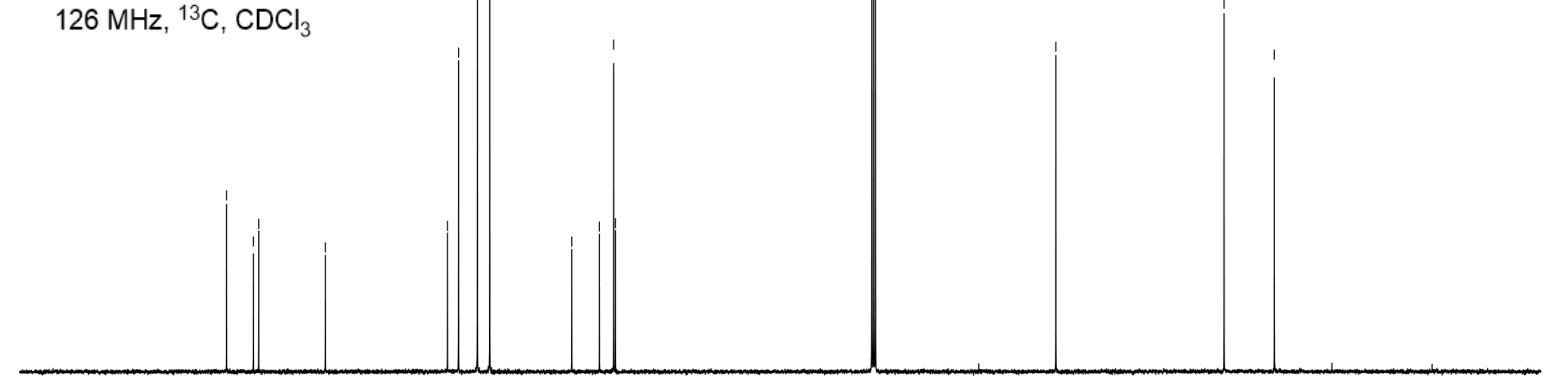

$\begin{array}{llllllllllllllllllllllll}190 & 180 & 170 & 160 & 150 & 140 & 130 & 120 & 110 & 100 & 90 & 80 & 70 & 60 & 50 & 40 & 30 & 20 & 10 & 0 & -10\end{array}$ 


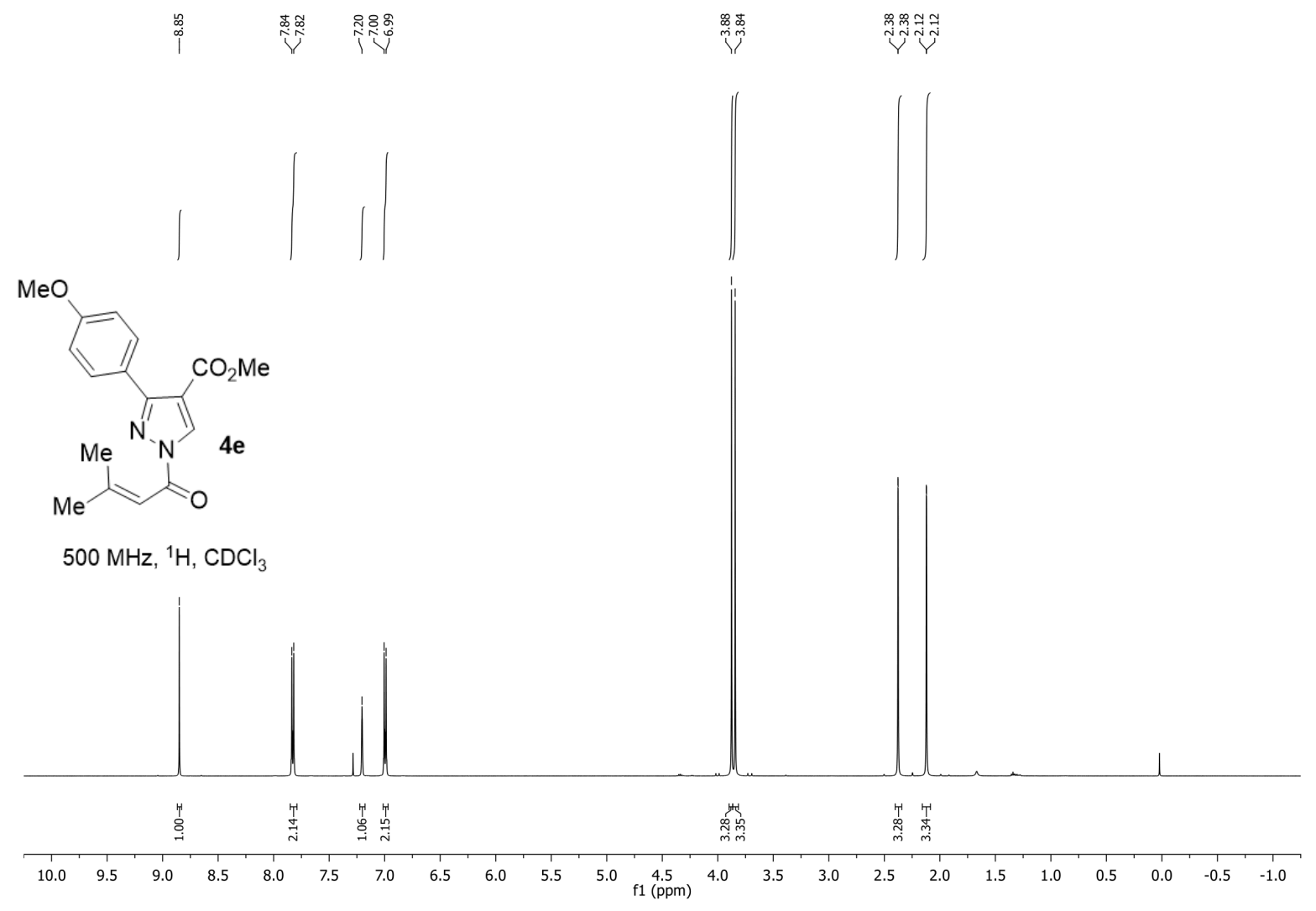

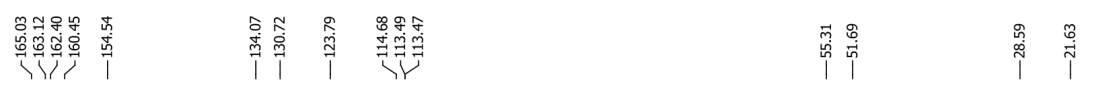

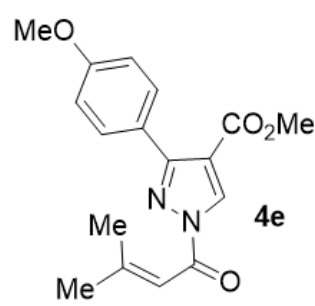

$126 \mathrm{MHz},{ }^{13} \mathrm{C}, \mathrm{CDCl}_{3}$

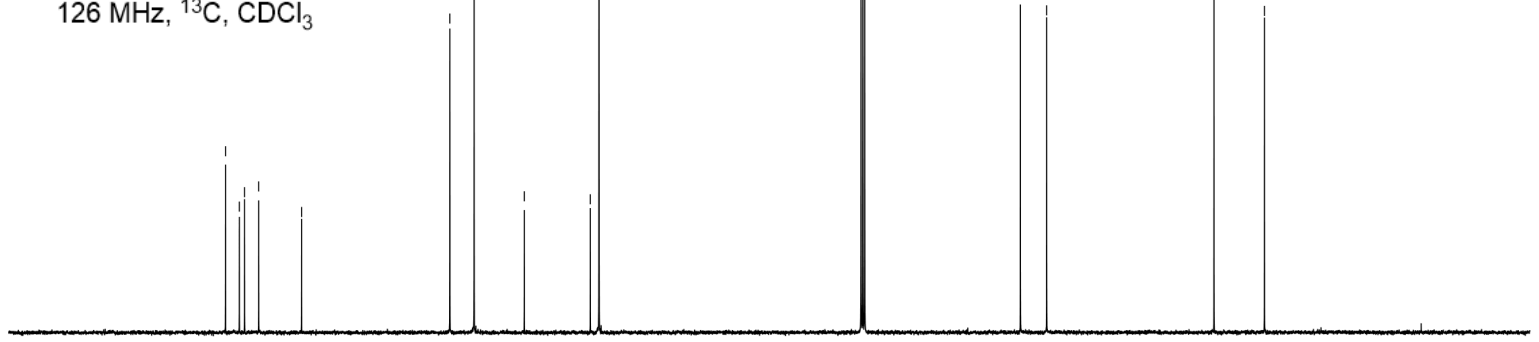

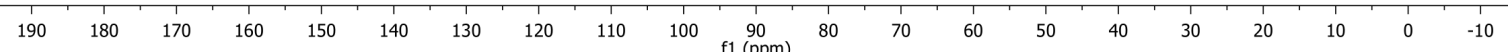




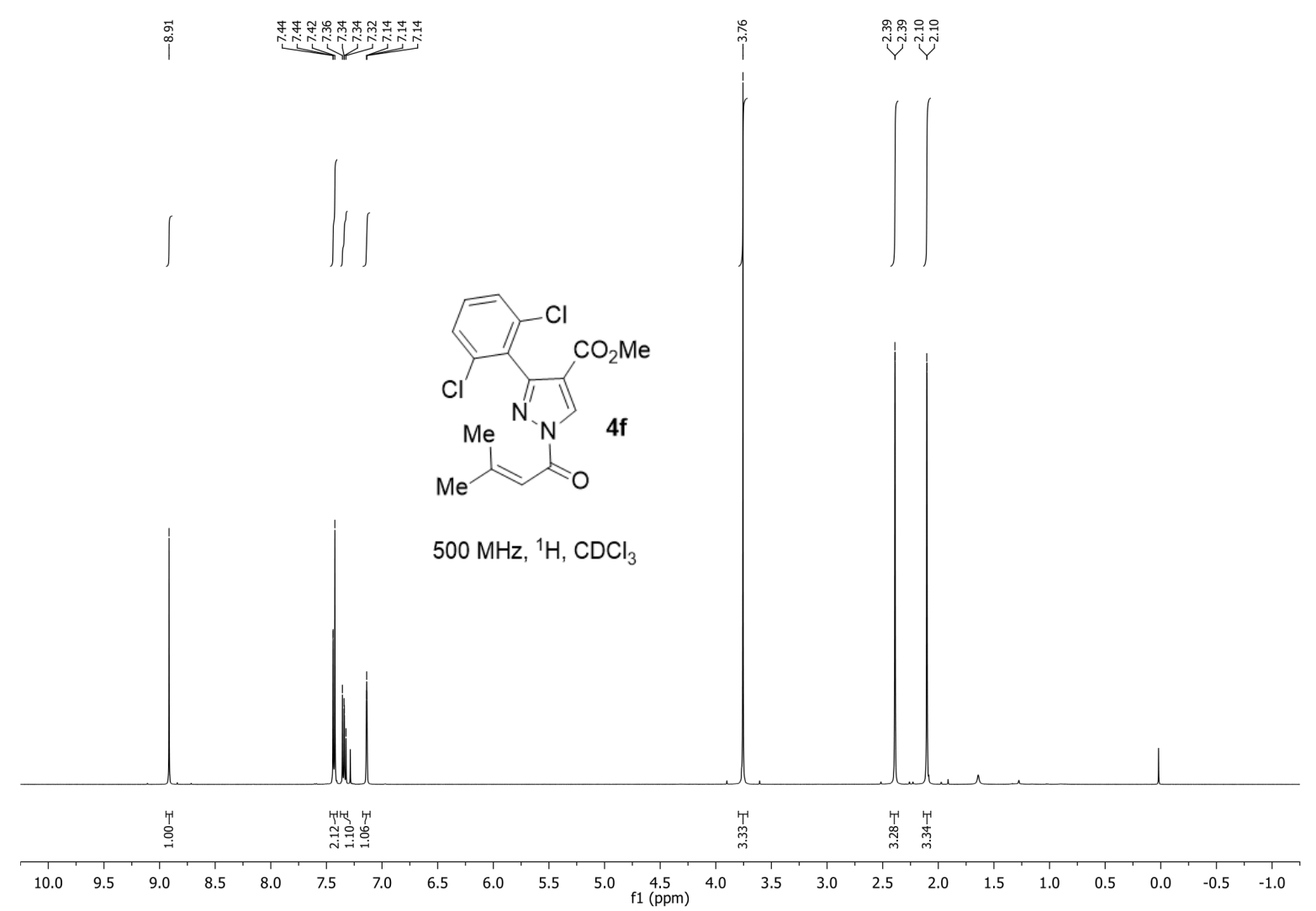

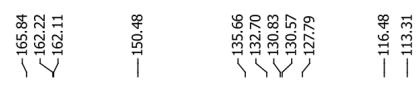
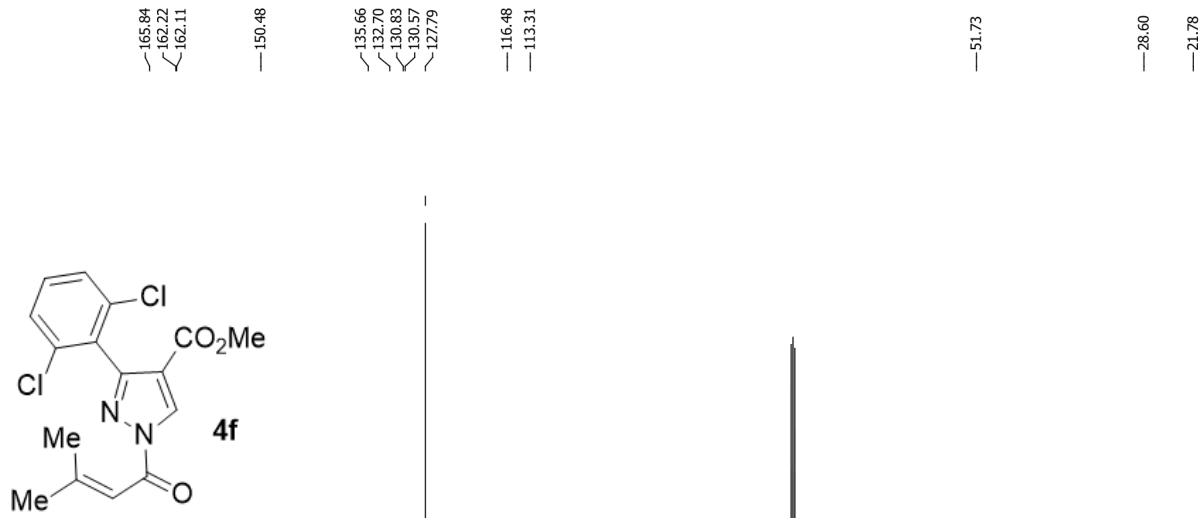

$126 \mathrm{MHz},{ }^{13} \mathrm{C}, \mathrm{CDCl}_{3}$

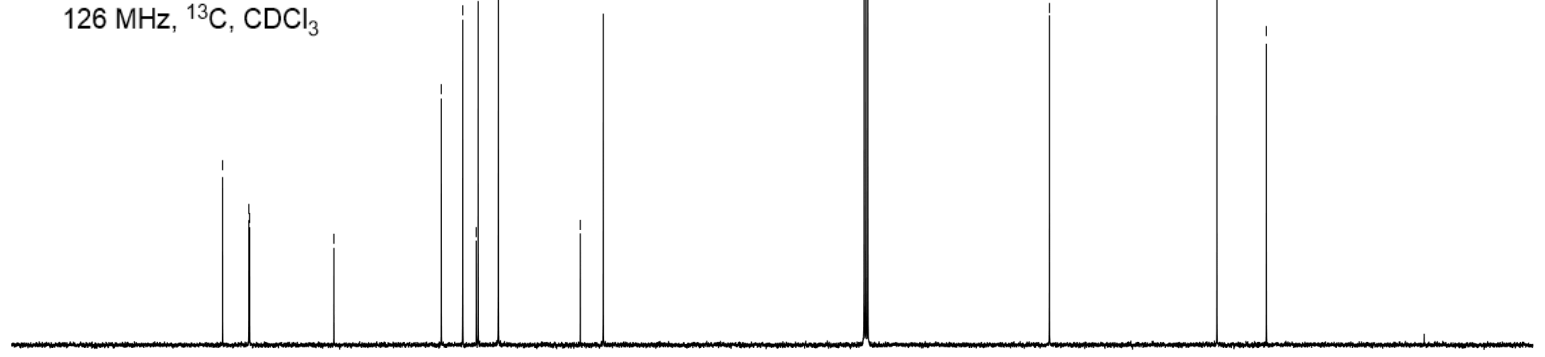

\begin{tabular}{|lllllllllllllllllllllllll}
190 & 180 & 170 & 160 & 150 & 140 & 130 & 120 & 110 & 100 & 90 & 80 & 70 & 60 & 50 & 40 & 30 & 20 & 10 & 0 & -10
\end{tabular} 

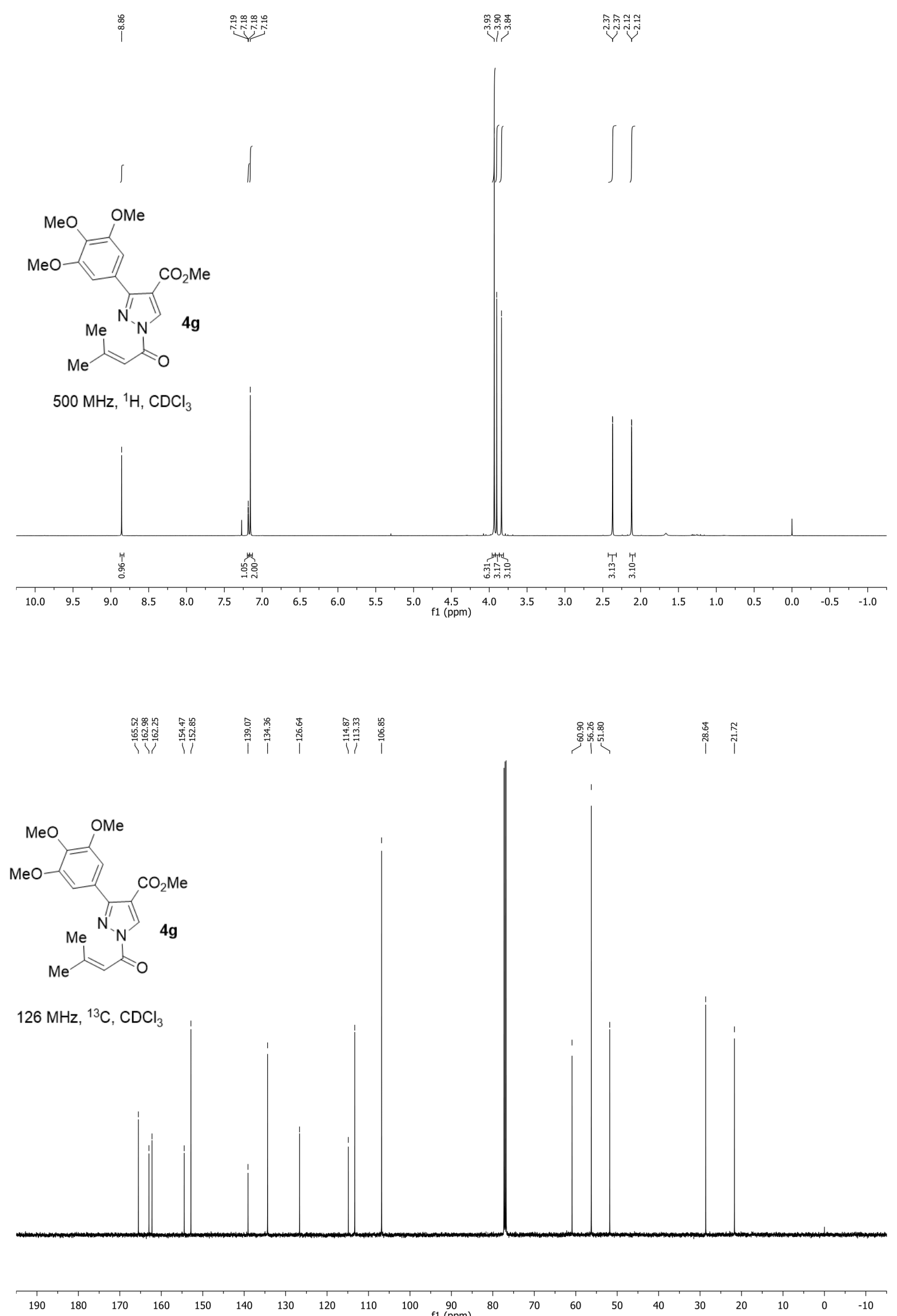

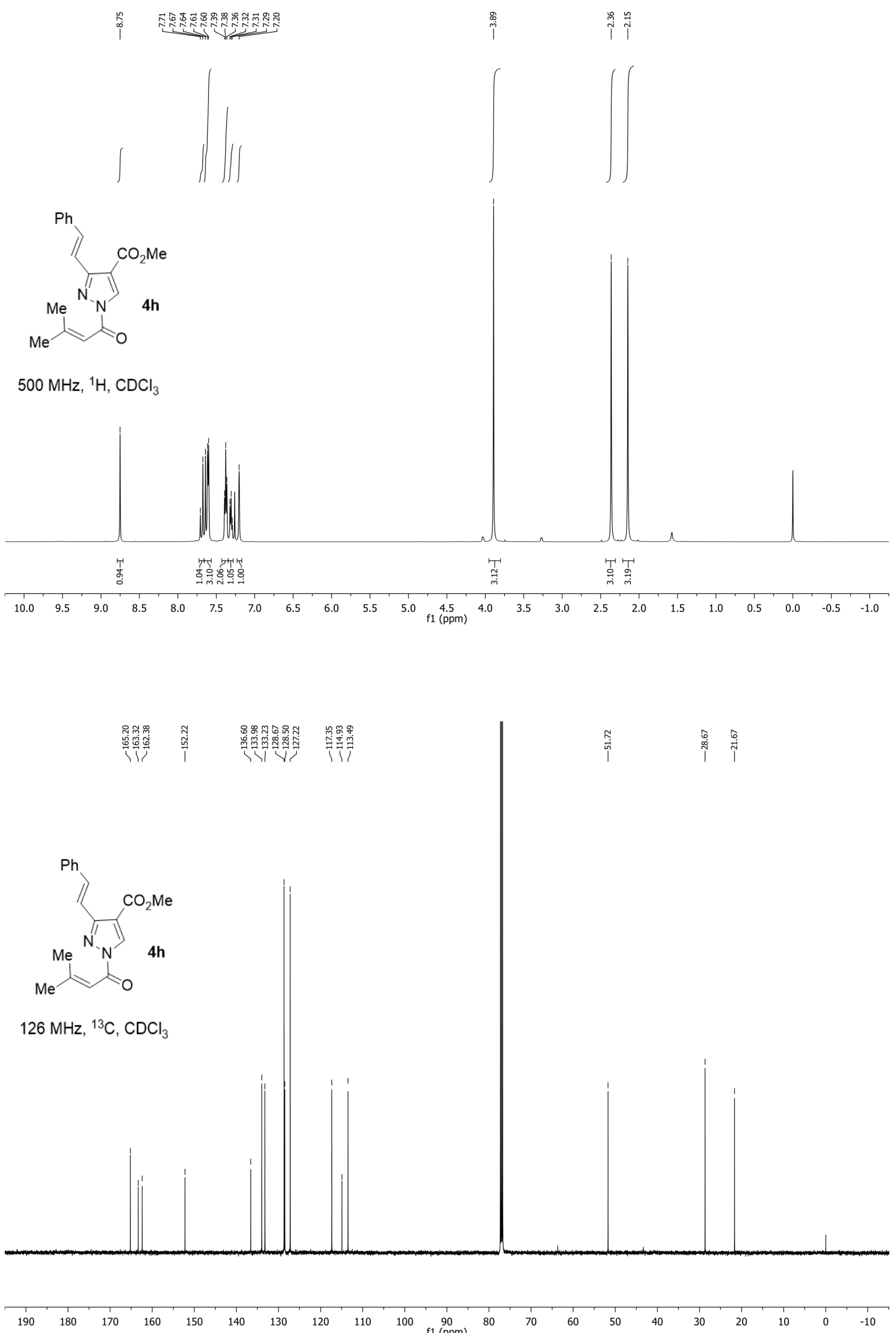

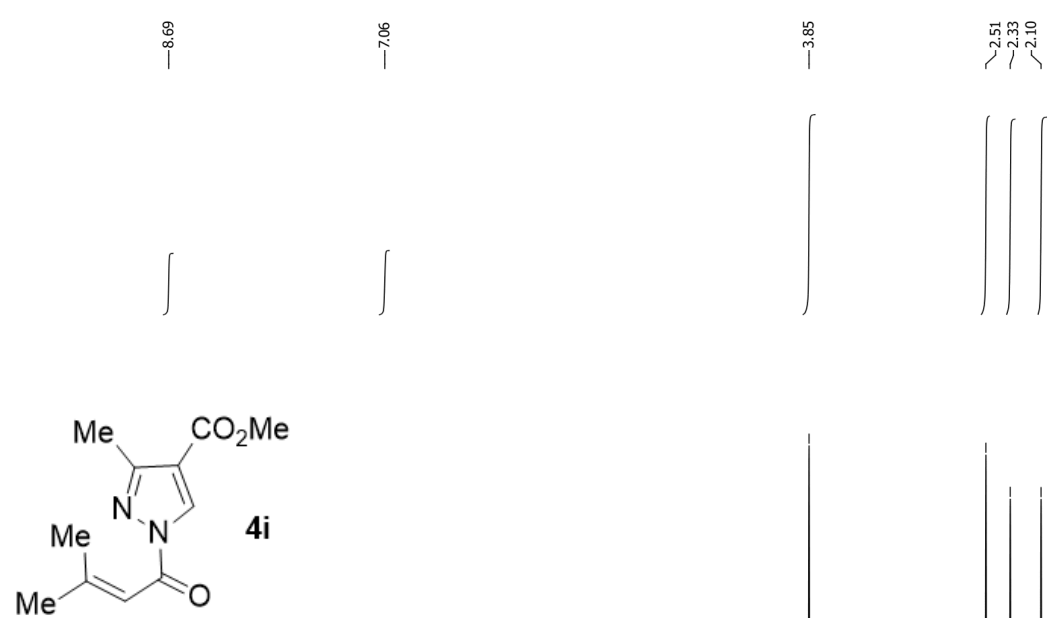

$500 \mathrm{MHz},{ }^{1} \mathrm{H}, \mathrm{CDCl}_{3}$

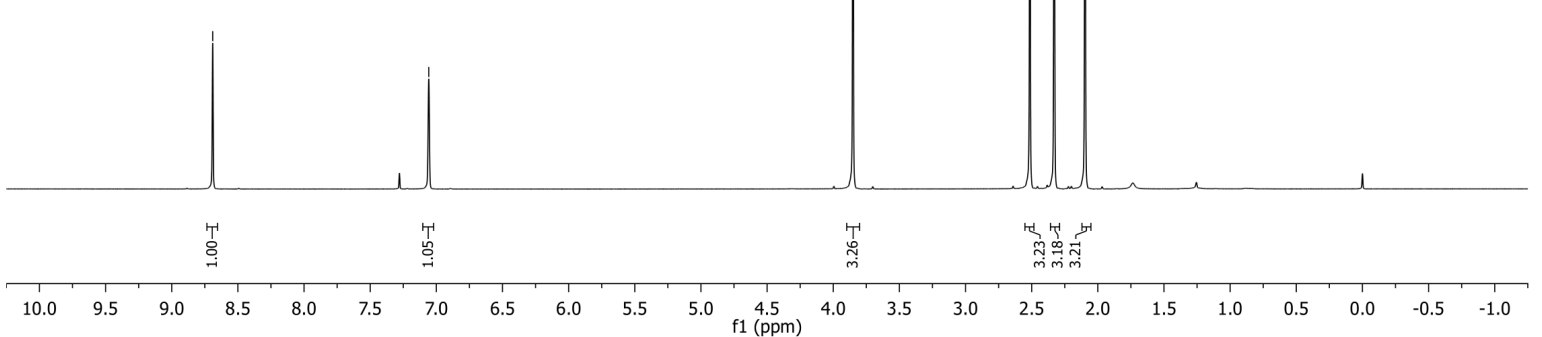

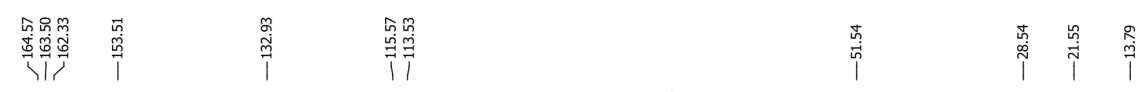<smiles></smiles>

$126 \mathrm{MHz},{ }^{13} \mathrm{C}, \mathrm{CDCl}_{3}$

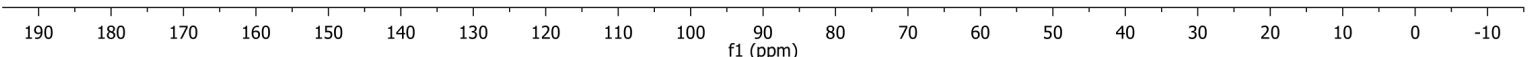



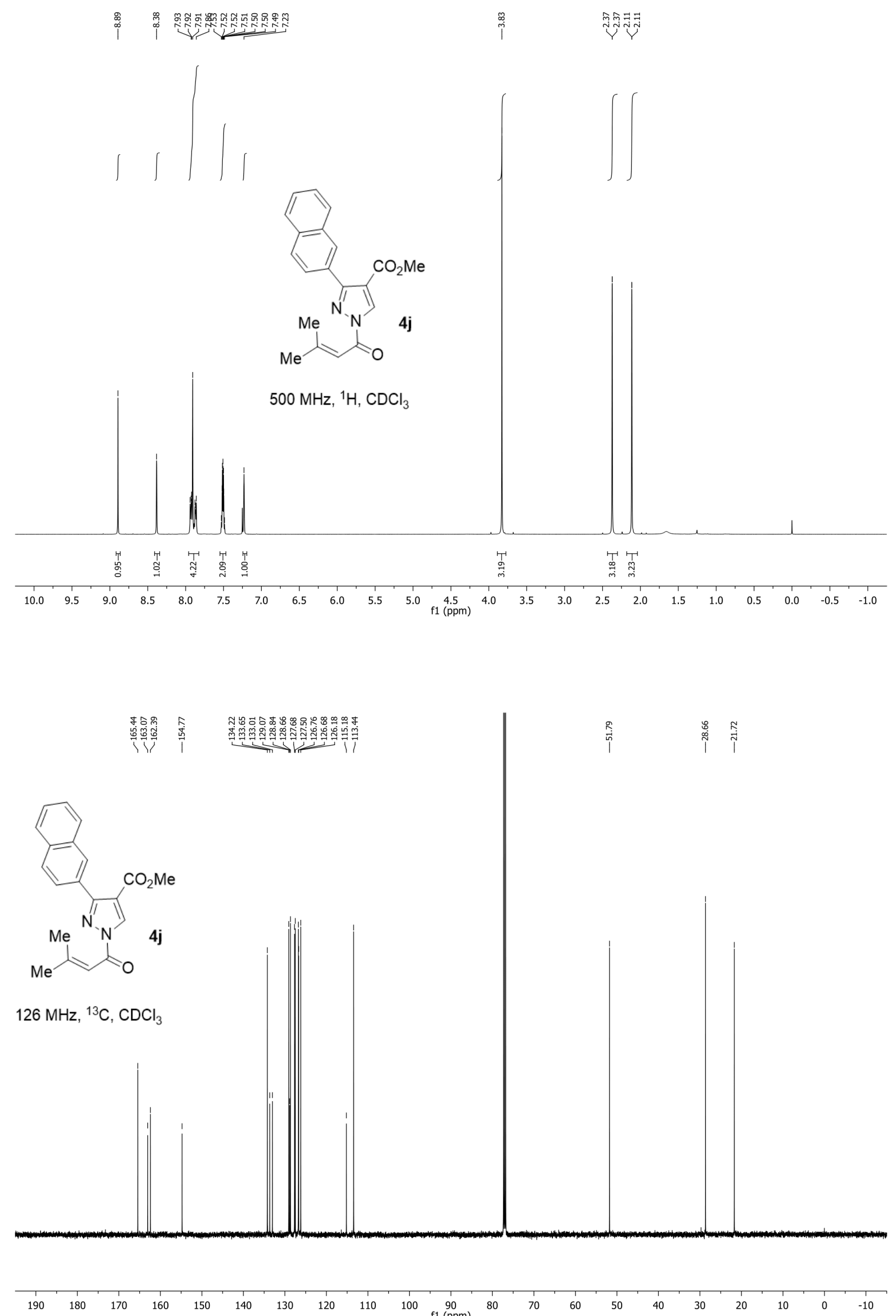

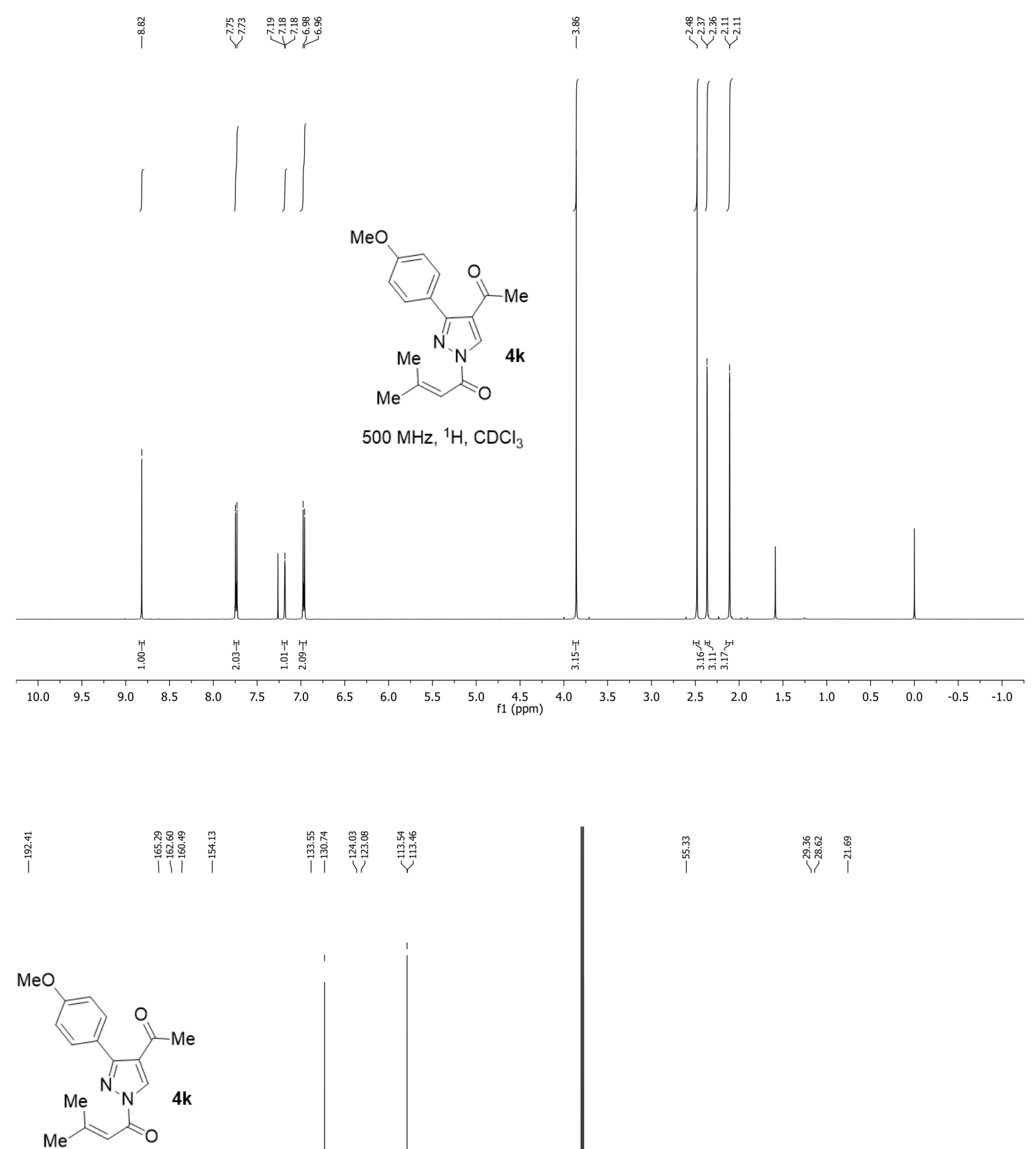

$126 \mathrm{MHz},{ }^{13} \mathrm{C}, \mathrm{CDCl}_{3}$
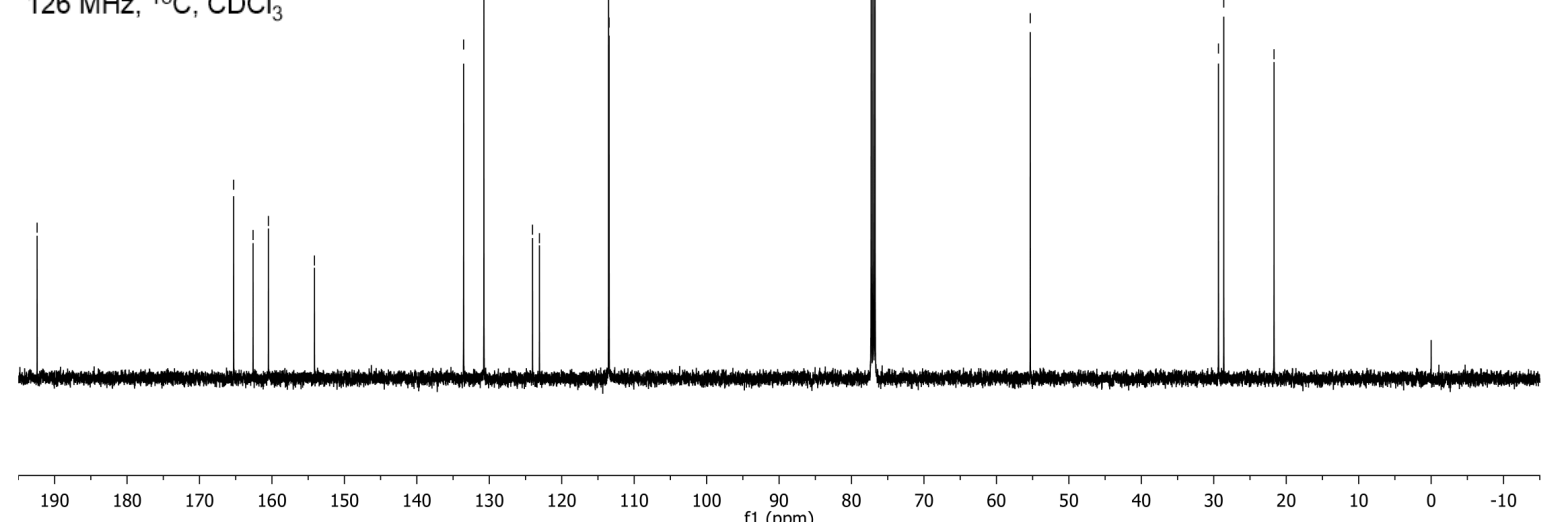


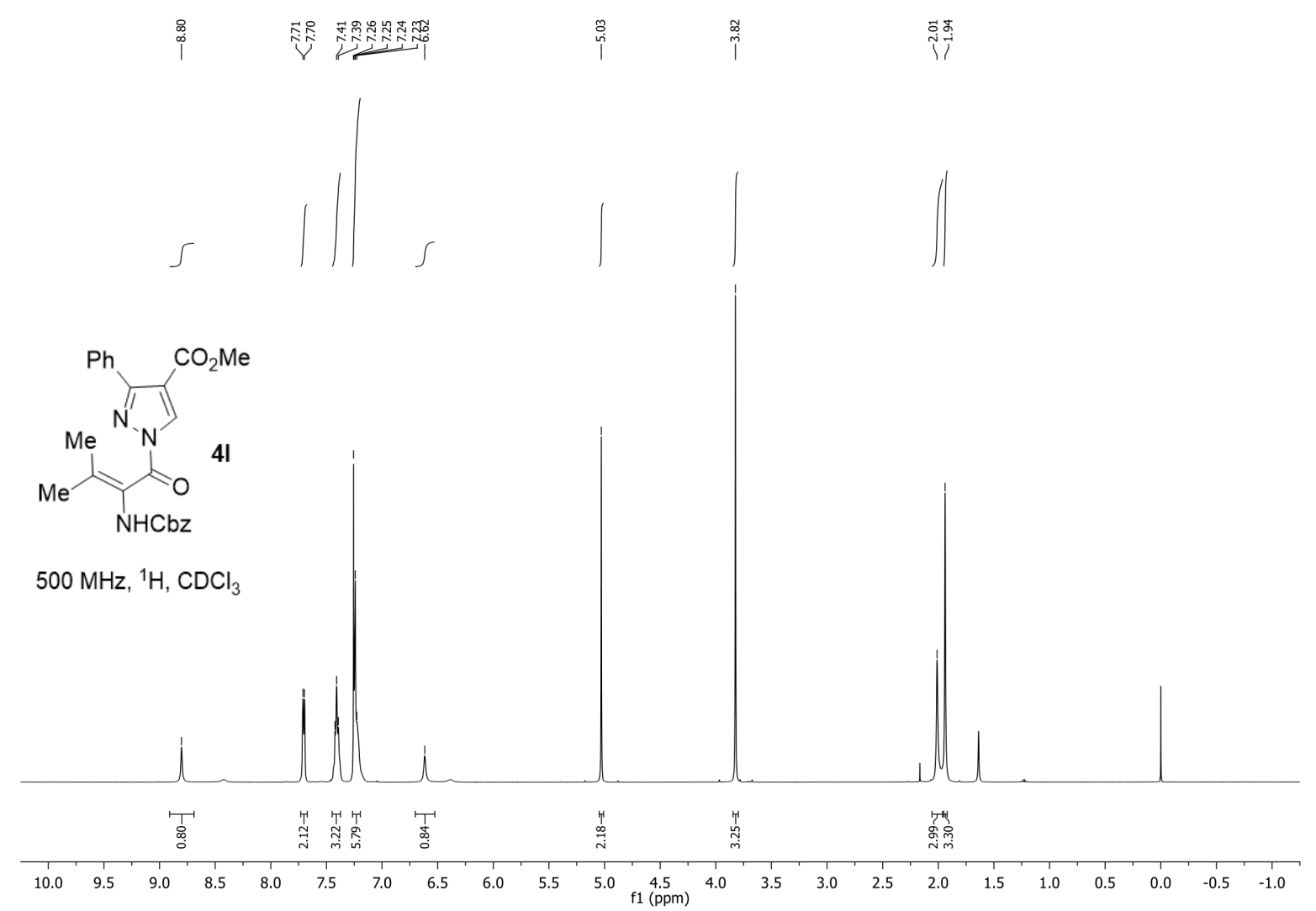

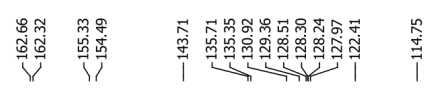

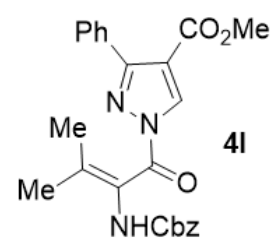

$126 \mathrm{MHz},{ }^{13} \mathrm{C}, \mathrm{CDCl}_{3}$
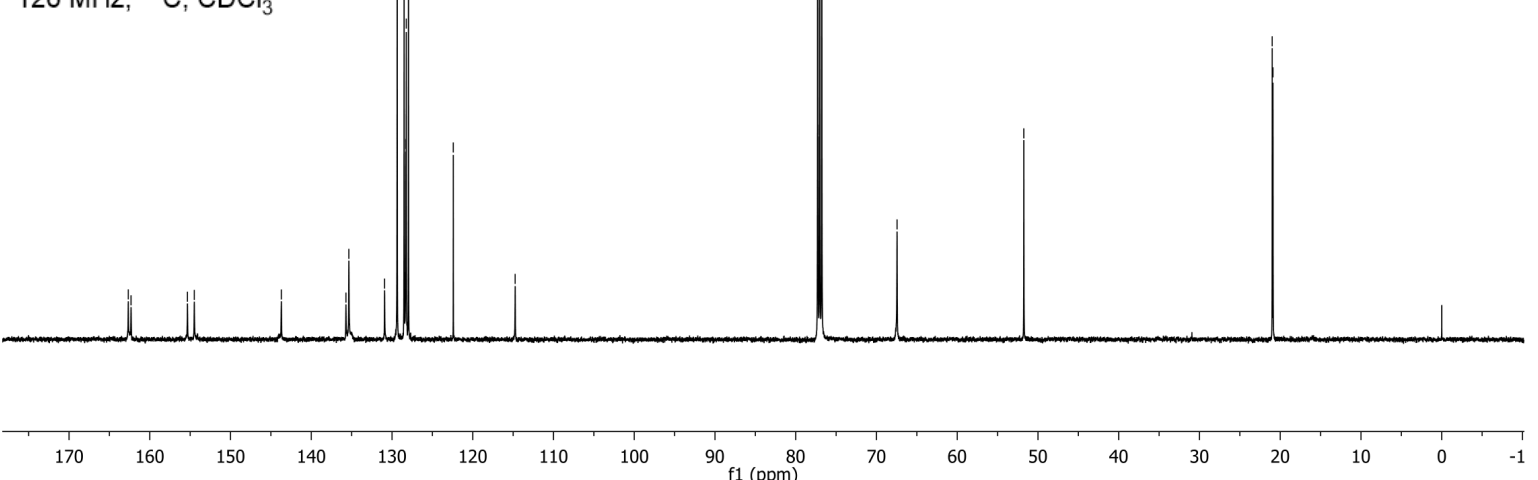

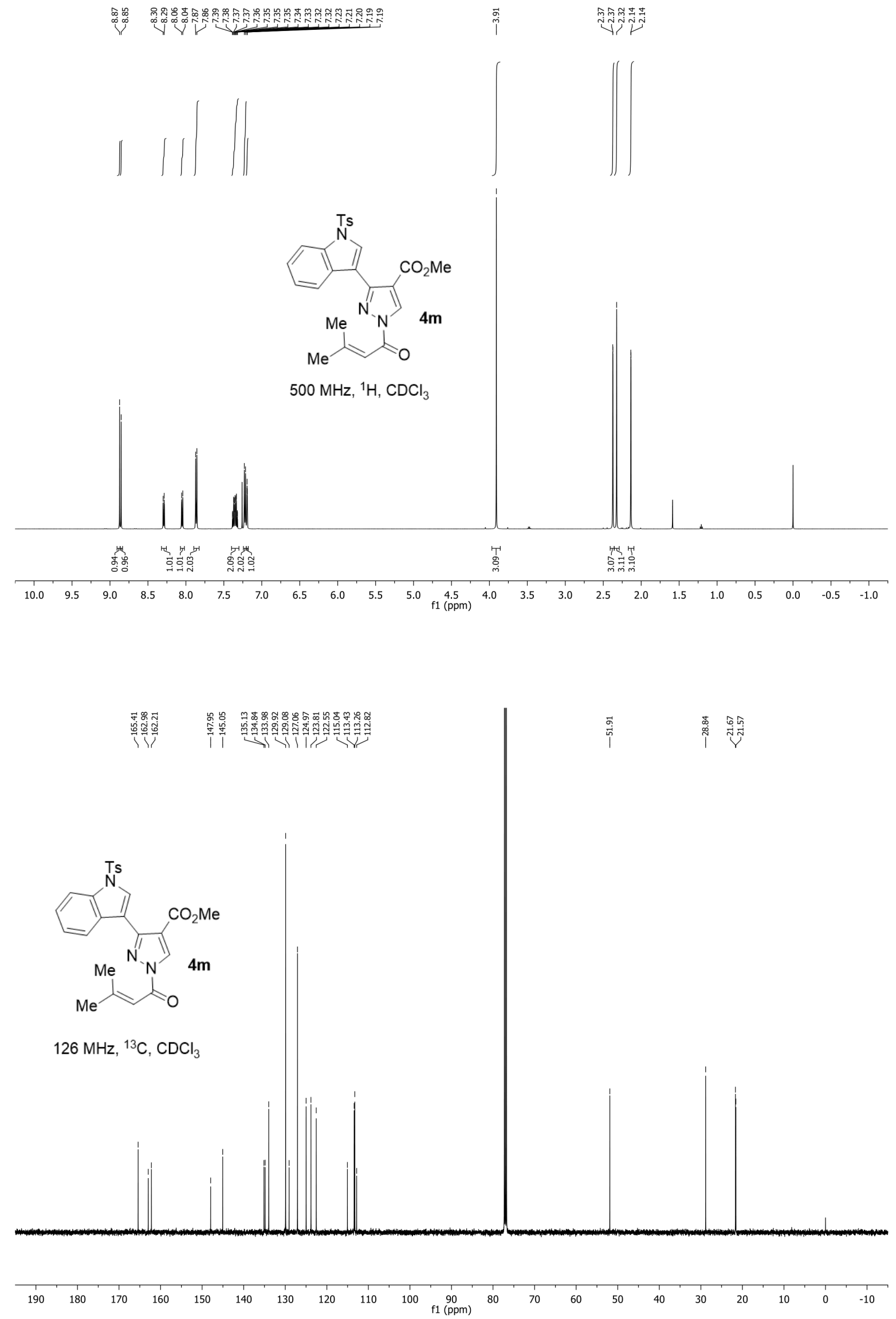

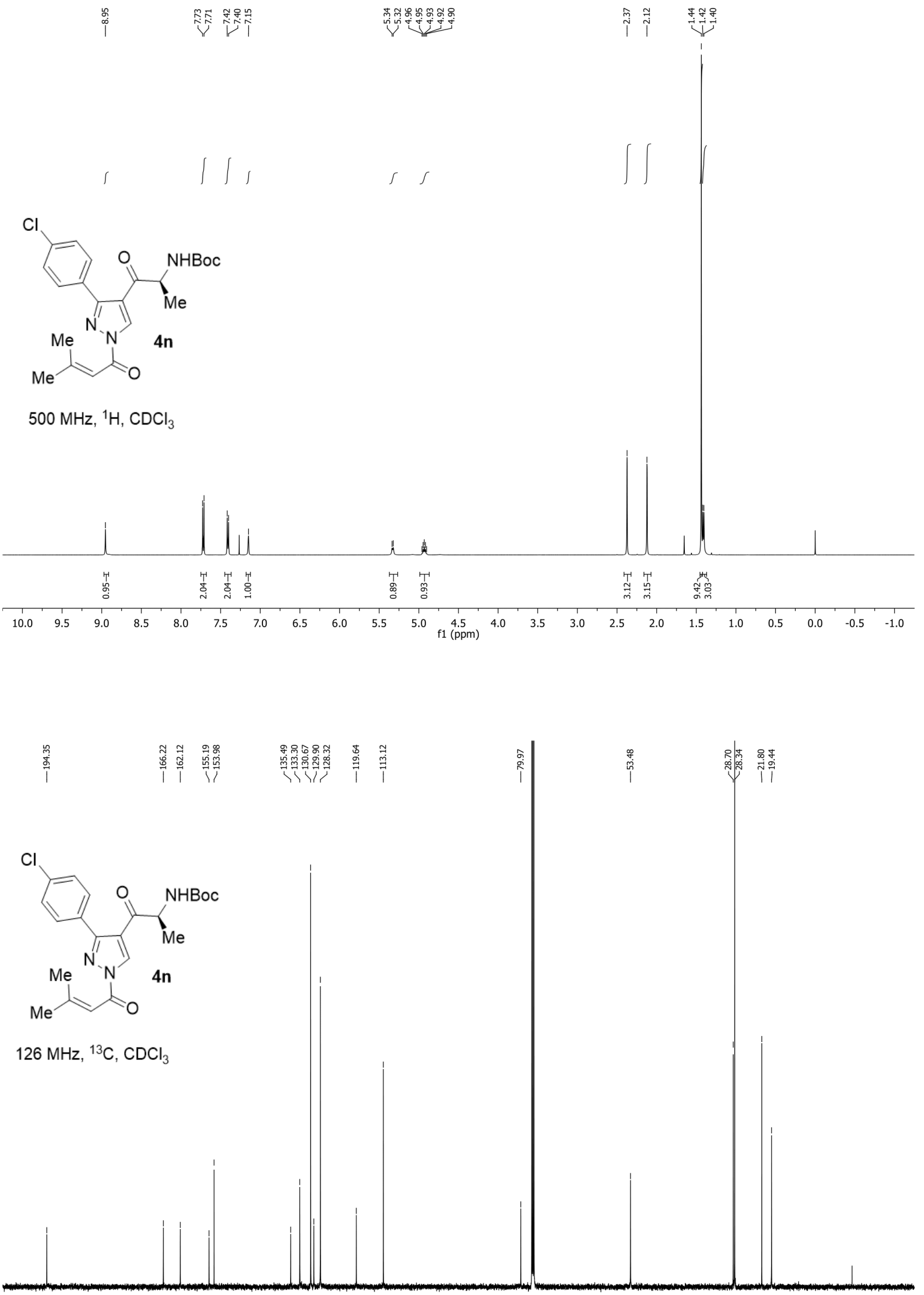

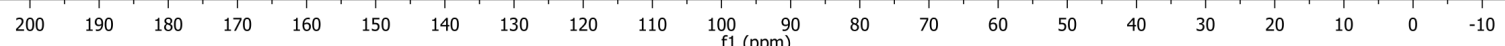



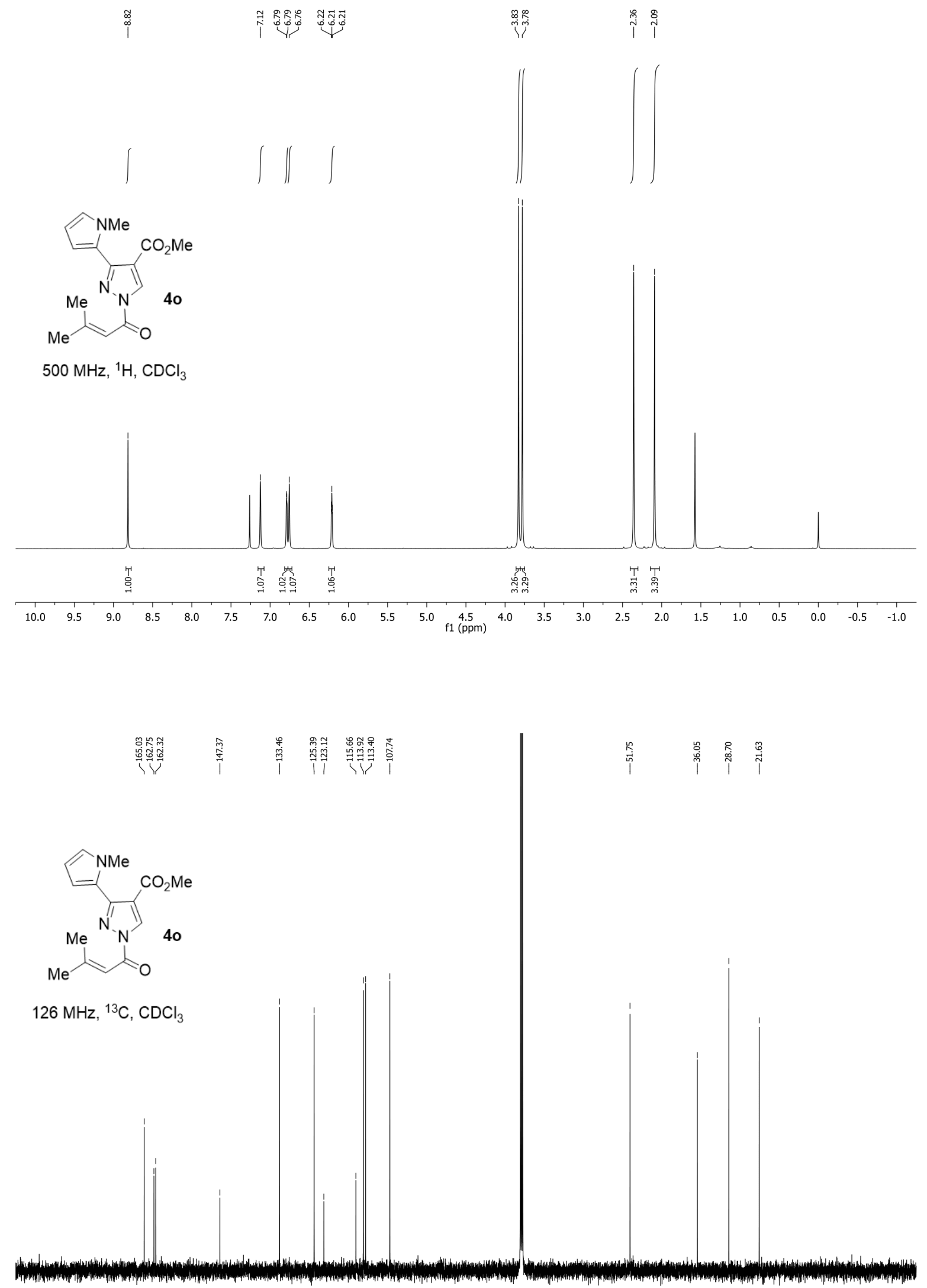

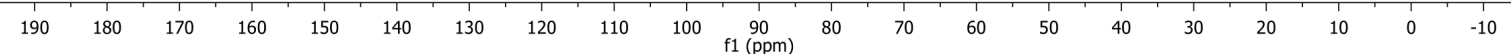



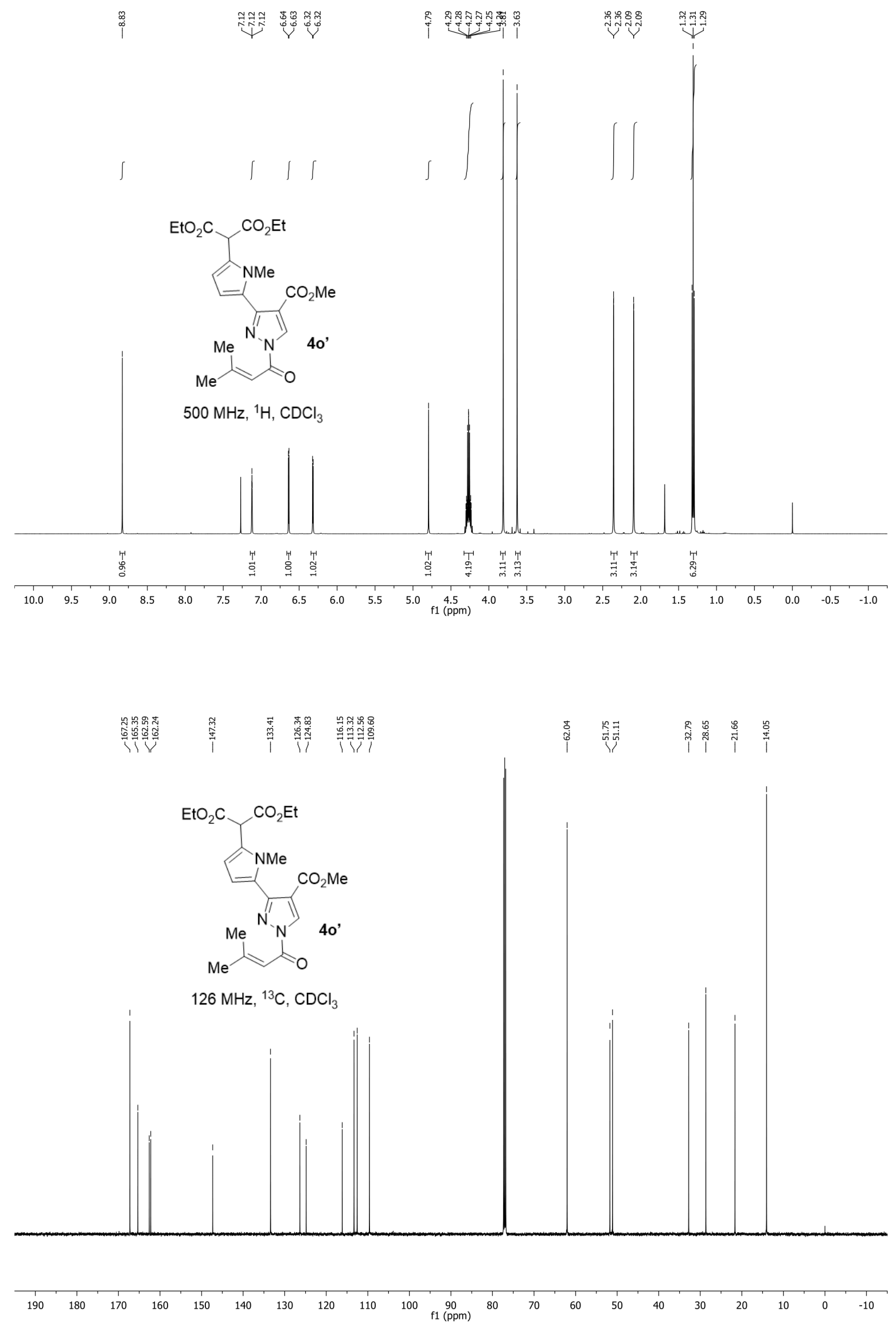


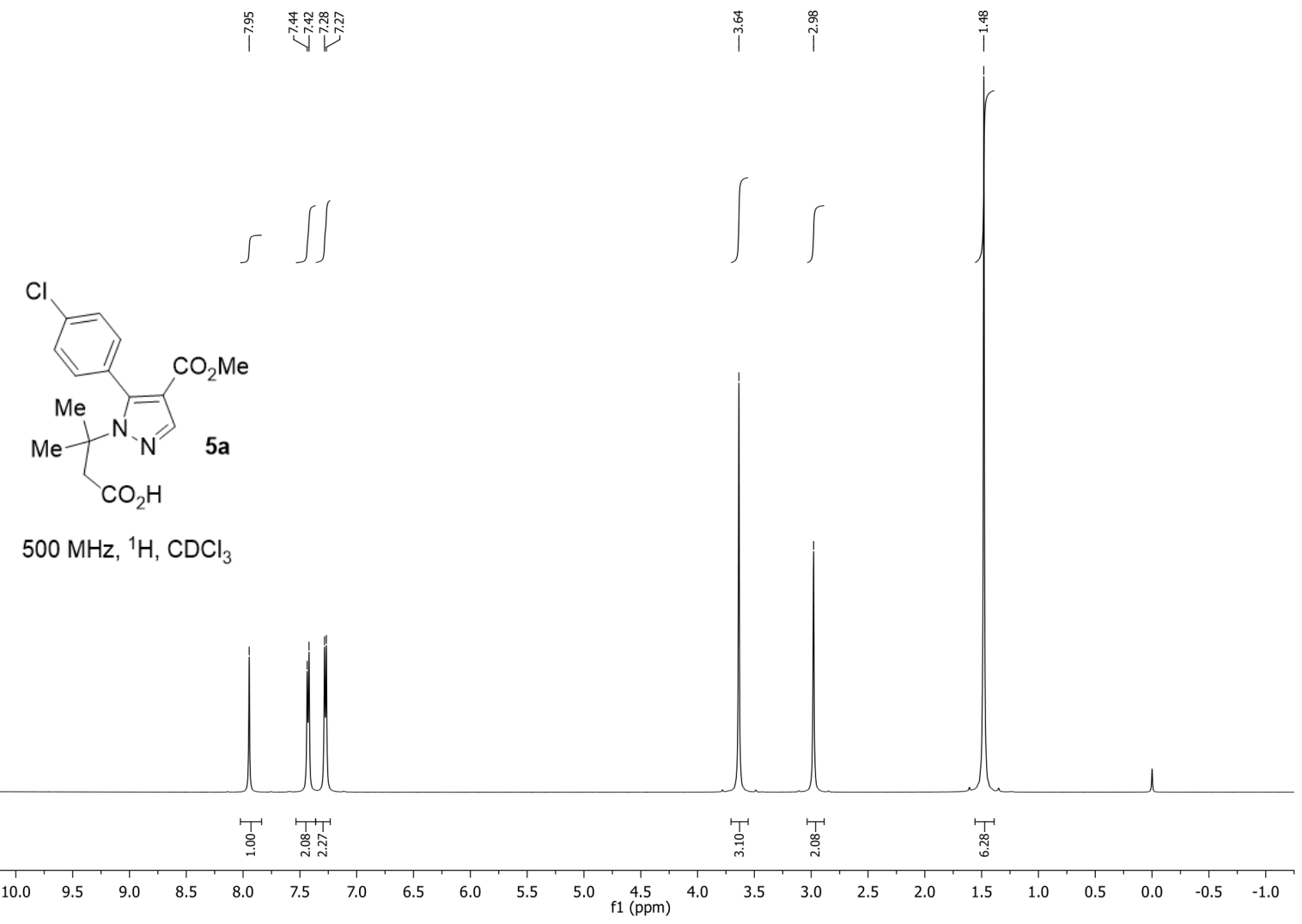

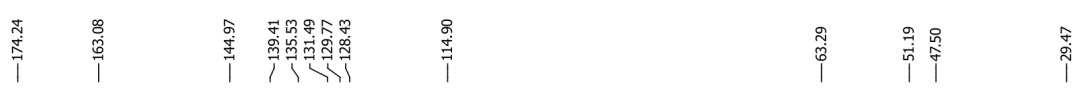

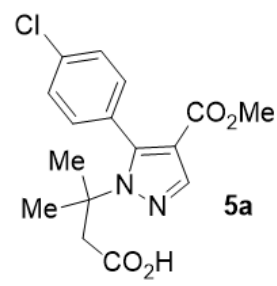

$126 \mathrm{MHz},{ }^{13} \mathrm{C}, \mathrm{CDCl}_{3}$

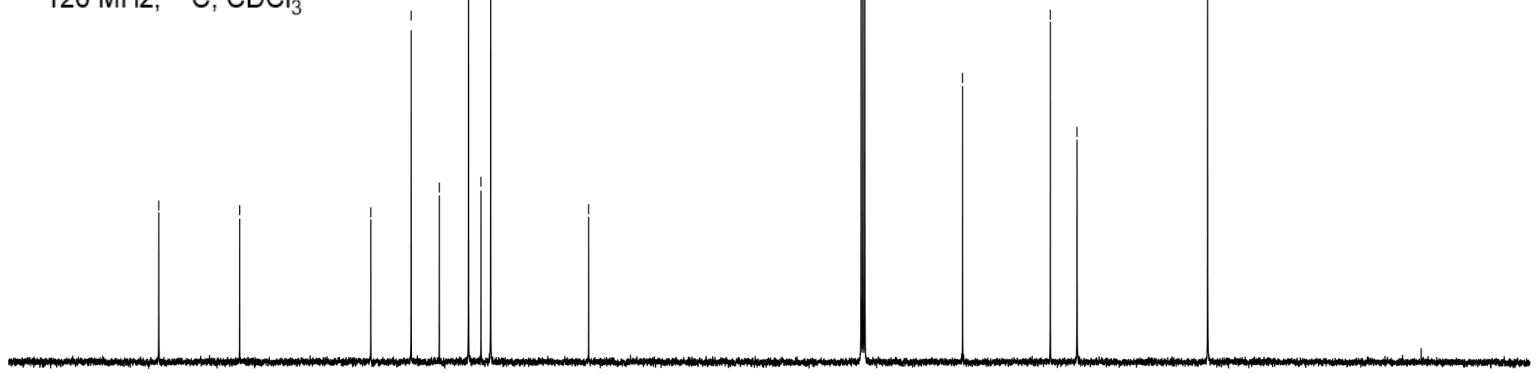

$\begin{array}{lllllllllllllllllllll}190 & 180 & 170 & 160 & 150 & 140 & 130 & 120 & 110 & 100 & 90 & 80 & 70 & 60 & 50 & 40 & 30 & 20 & 10 & 0 & -10\end{array}$ 

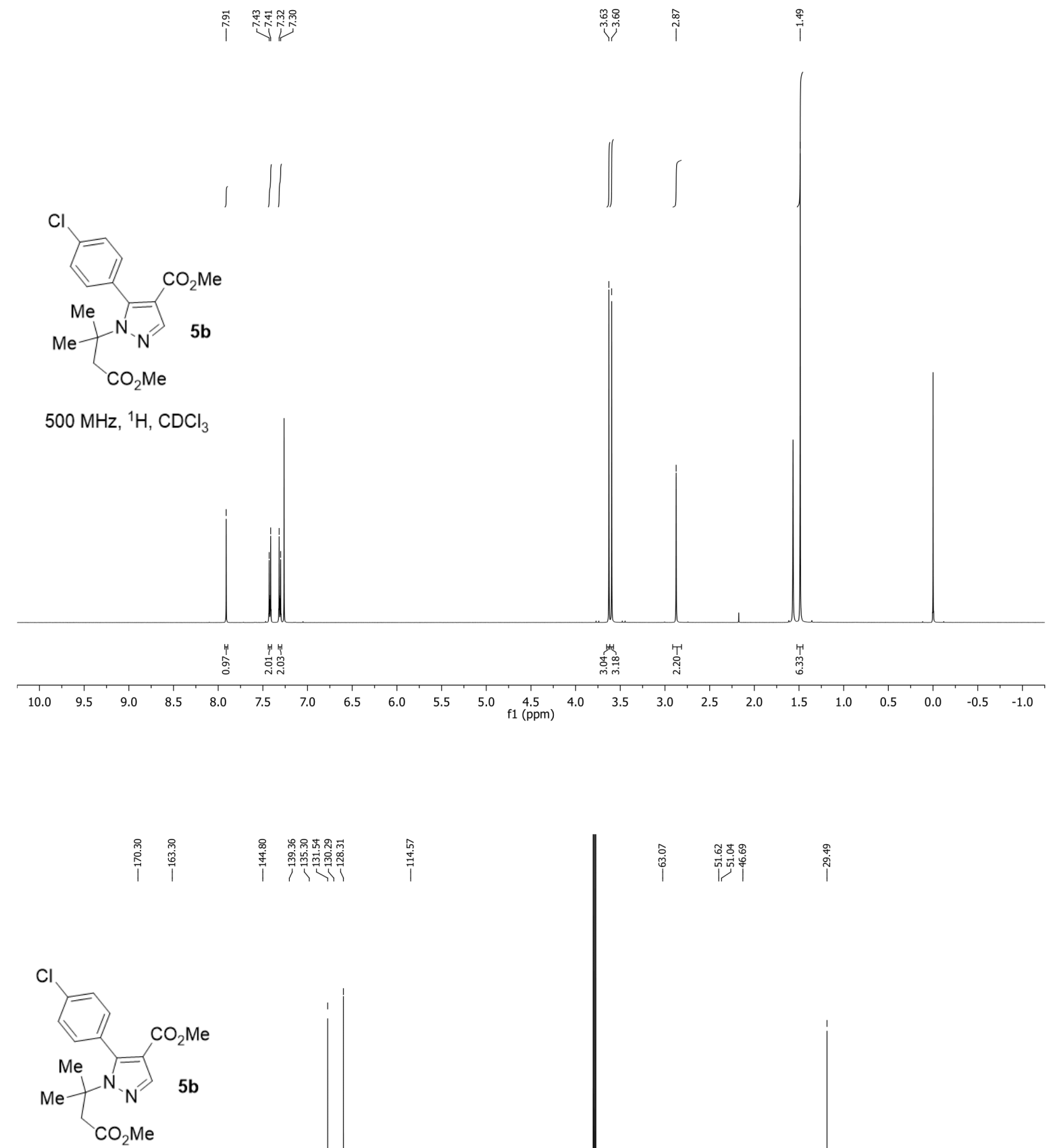

$126 \mathrm{MHz},{ }^{13} \mathrm{C}, \mathrm{CDCl}_{3}$

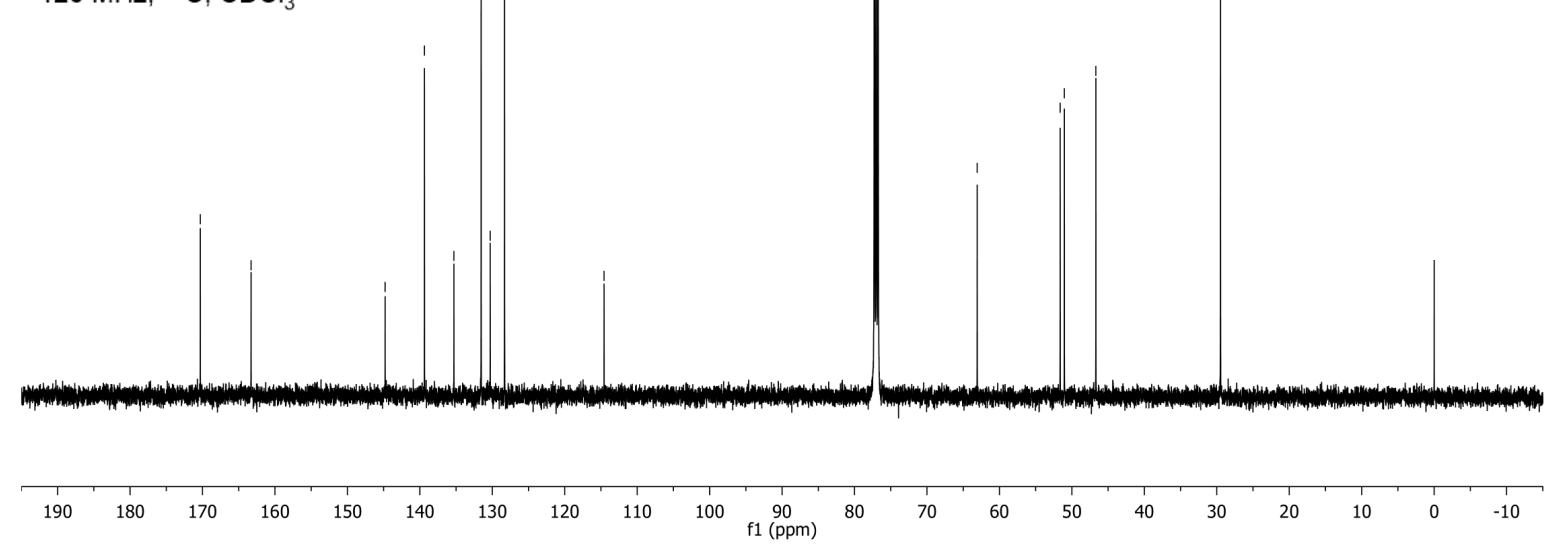




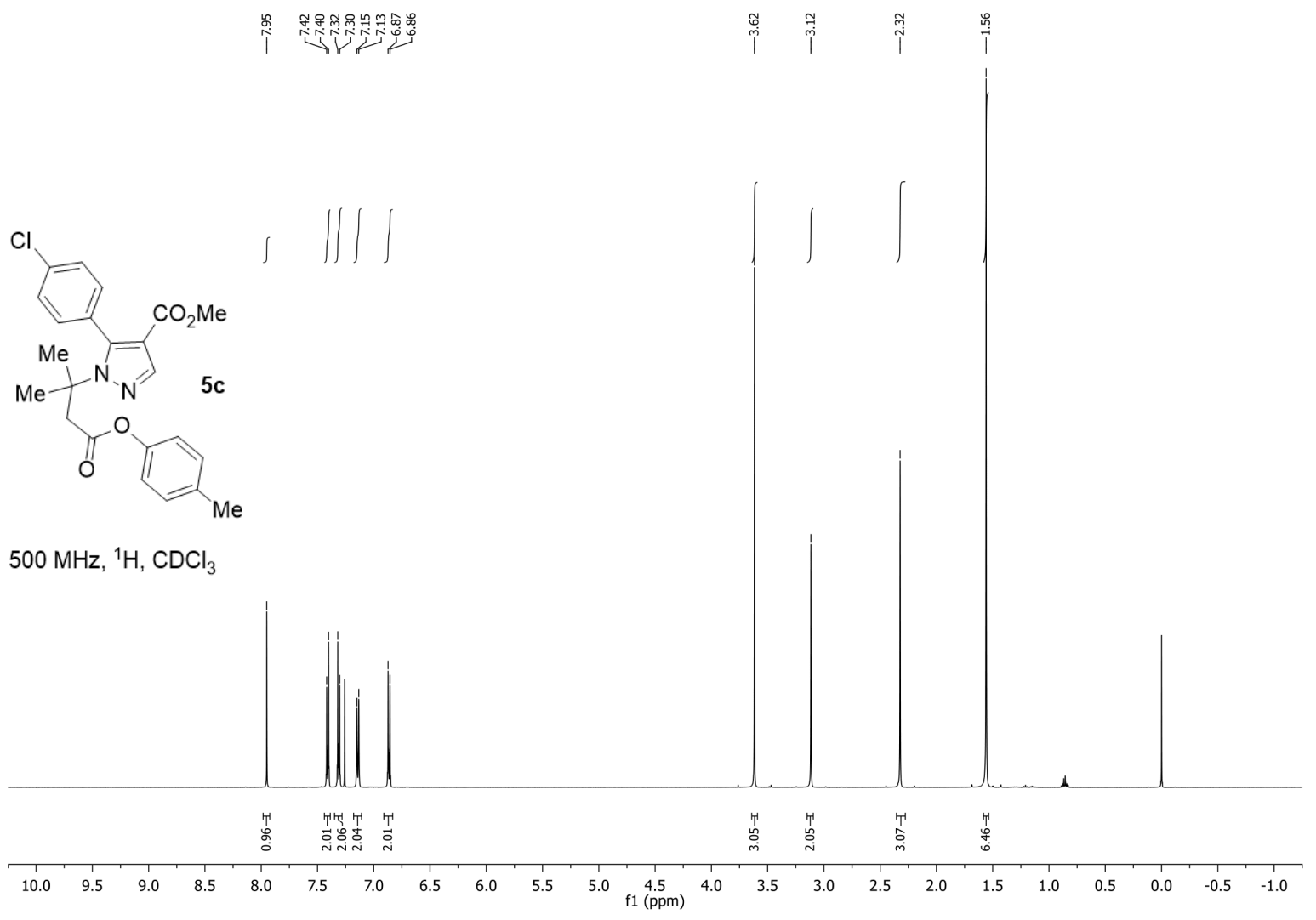

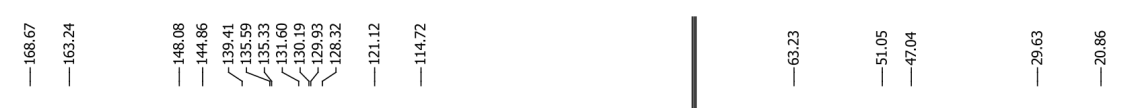

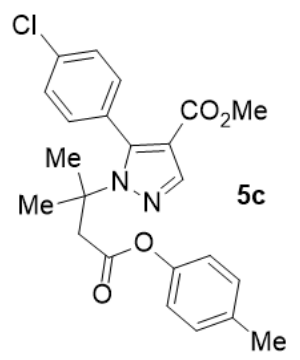

$126 \mathrm{MHz},{ }^{13} \mathrm{C}, \mathrm{CDCl}_{3}$

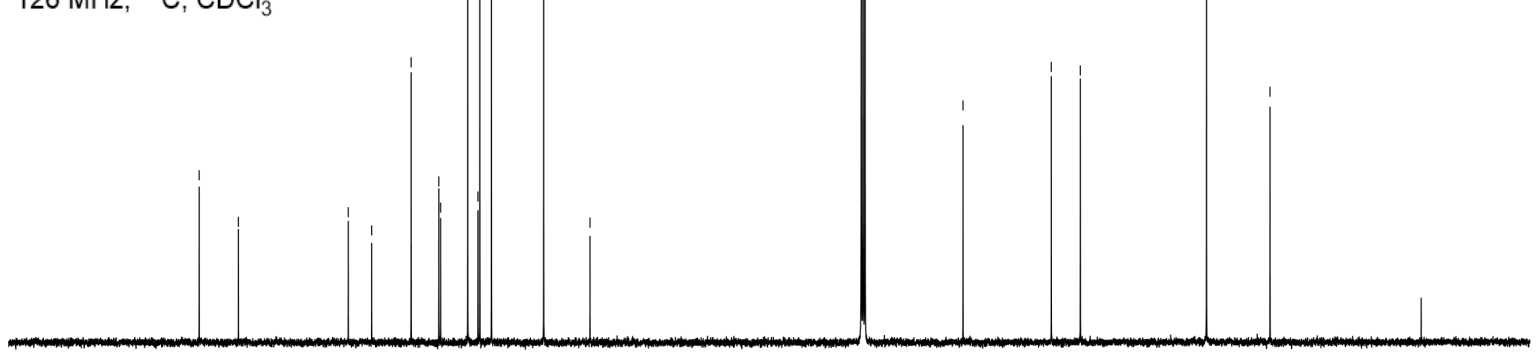

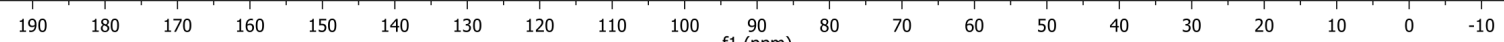




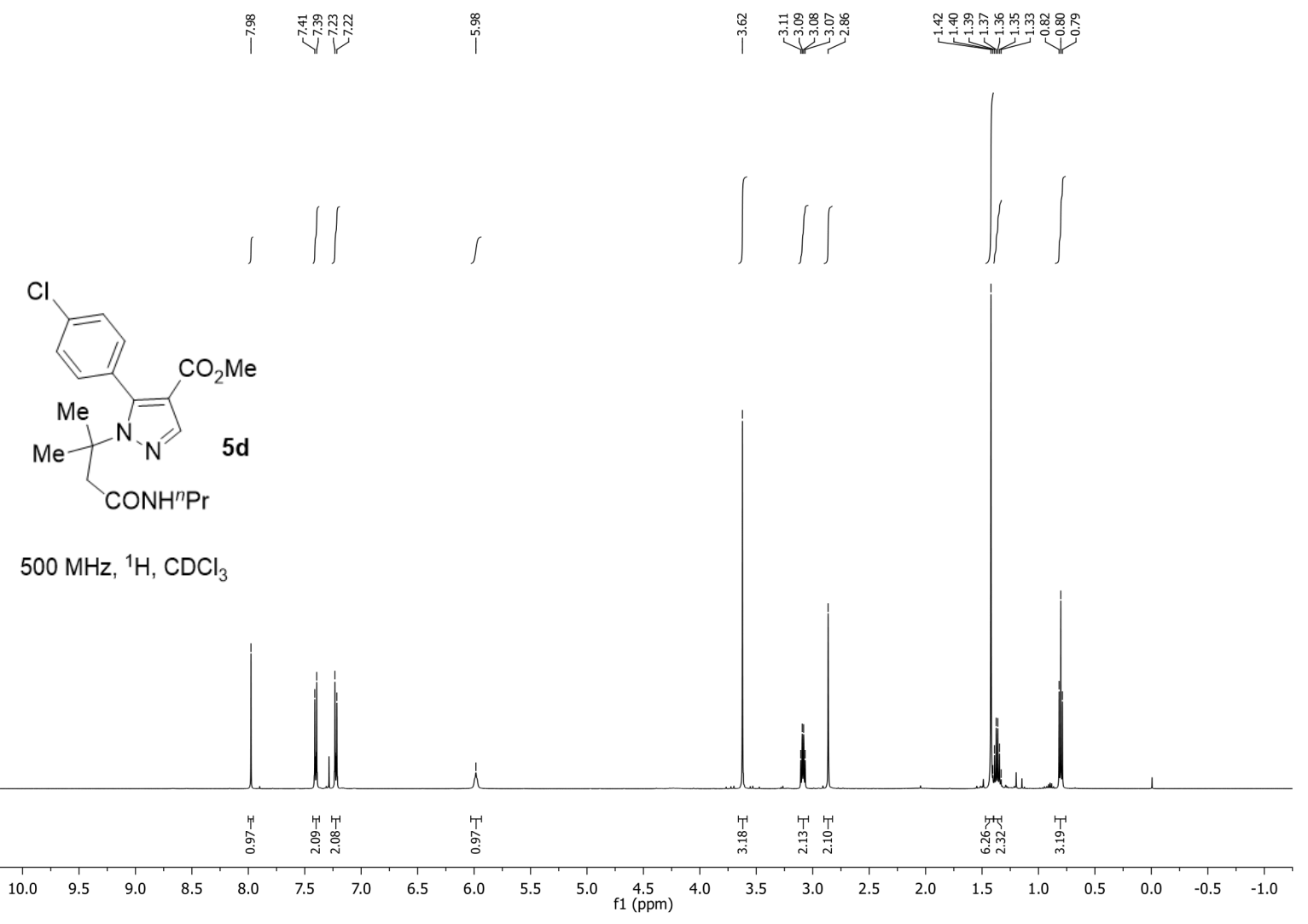

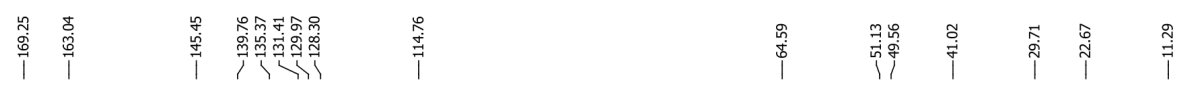

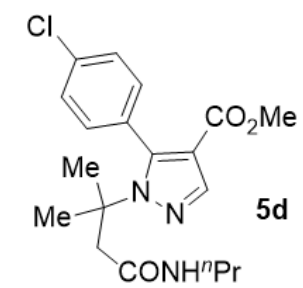

$126 \mathrm{MHz},{ }^{13} \mathrm{C}, \mathrm{CDCl}_{3}$

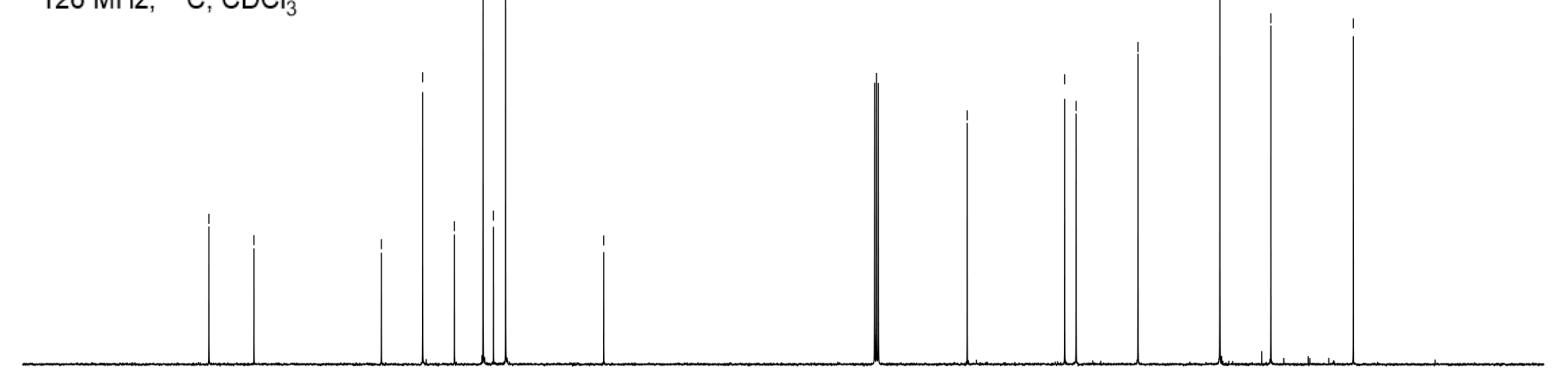

$\begin{array}{llllllllllllllllllllllll}190 & 180 & 170 & 160 & 150 & 140 & 130 & 120 & 110 & 100 & 90 & 80 & 70 & 60 & 50 & 40 & 30 & 20 & 10 & 0 & -10\end{array}$ 


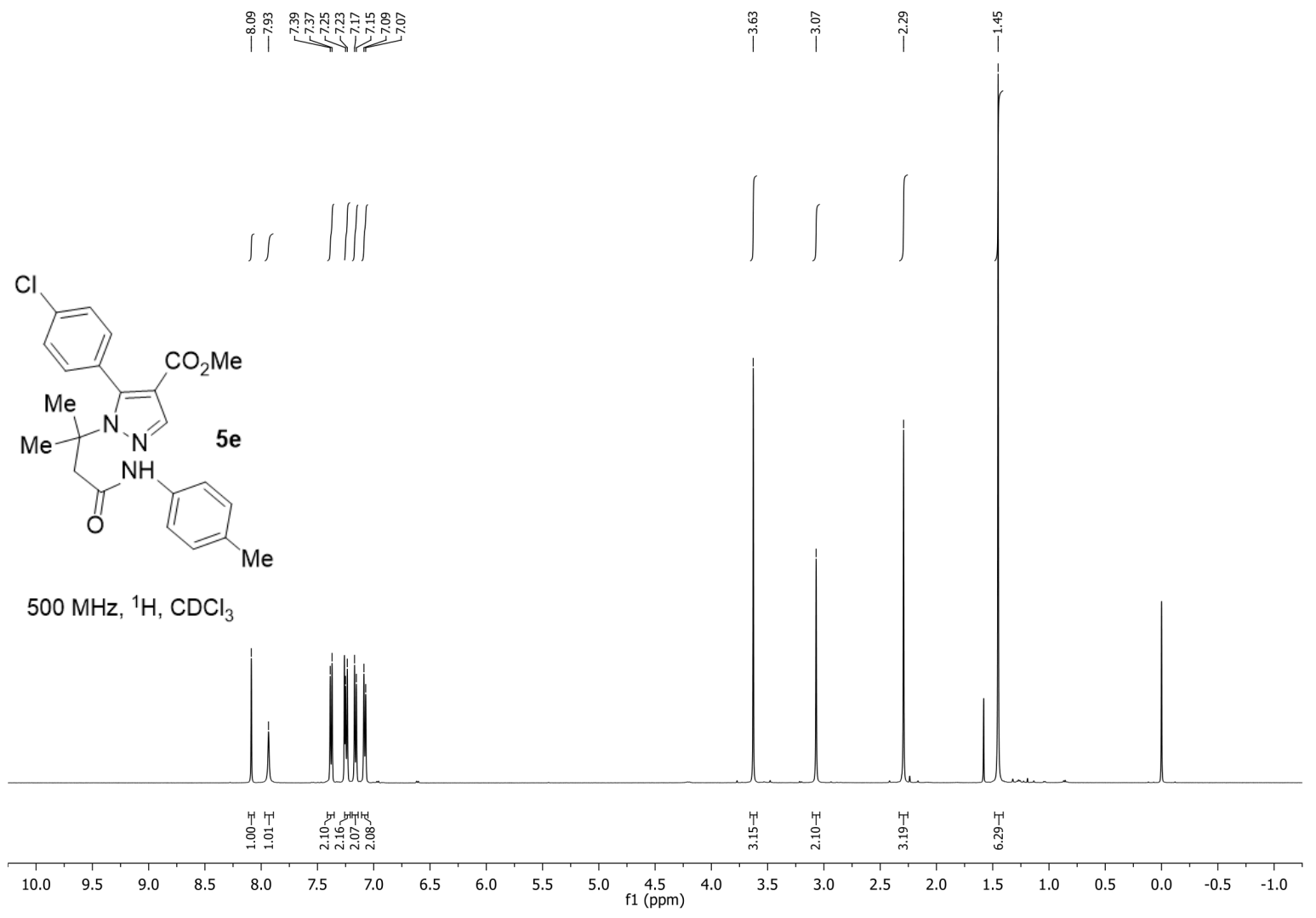

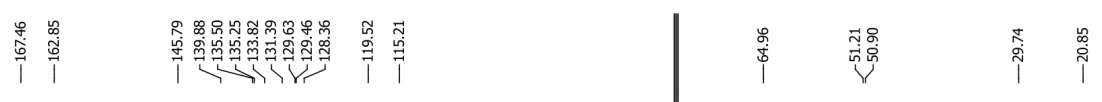

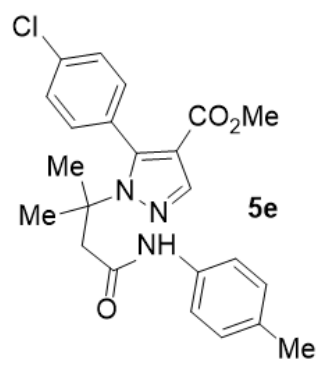

$126 \mathrm{MHz},{ }^{13} \mathrm{C}, \mathrm{CDCl}_{3}$

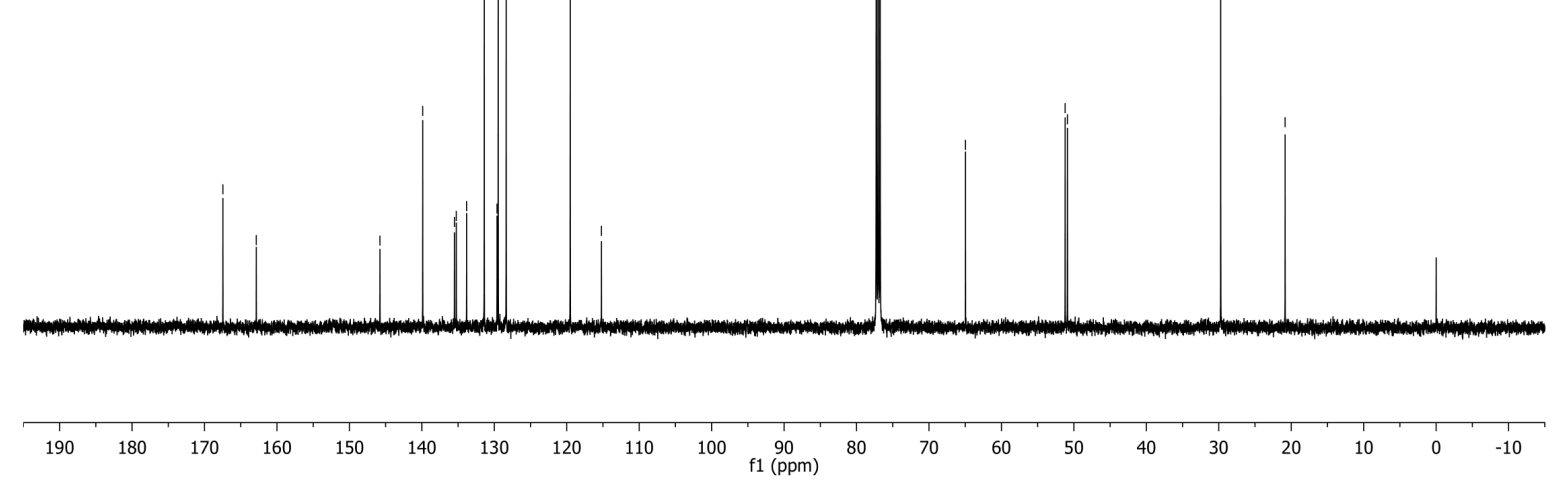



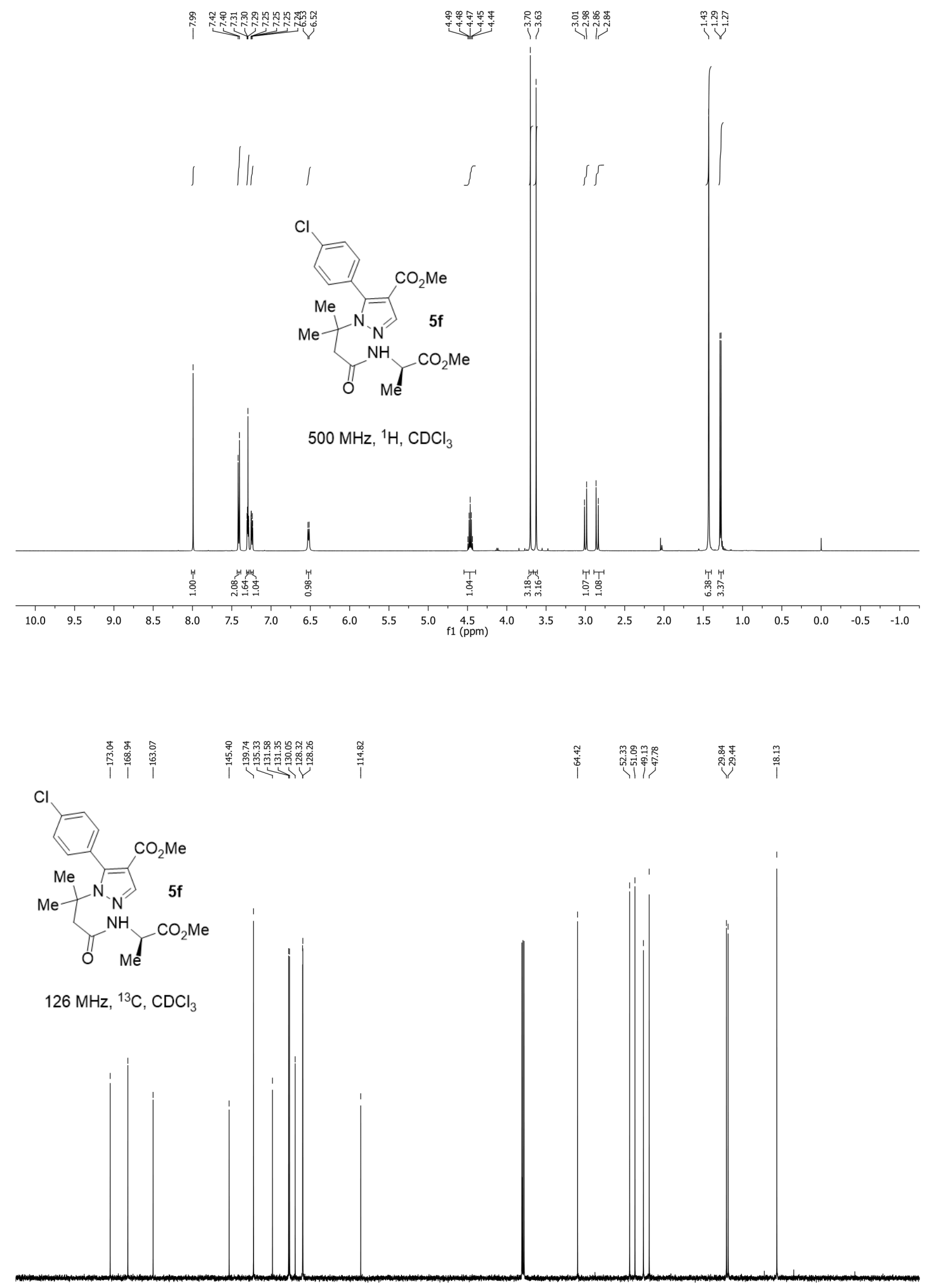

$\begin{array}{lllllllllllllllllllllll}190 & 180 & 170 & 160 & 150 & 140 & 130 & 120 & 110 & 100 & 90 & 80 & 70 & 60 & 50 & 40 & 30 & 20 & 10 & 0 & -10\end{array}$ 

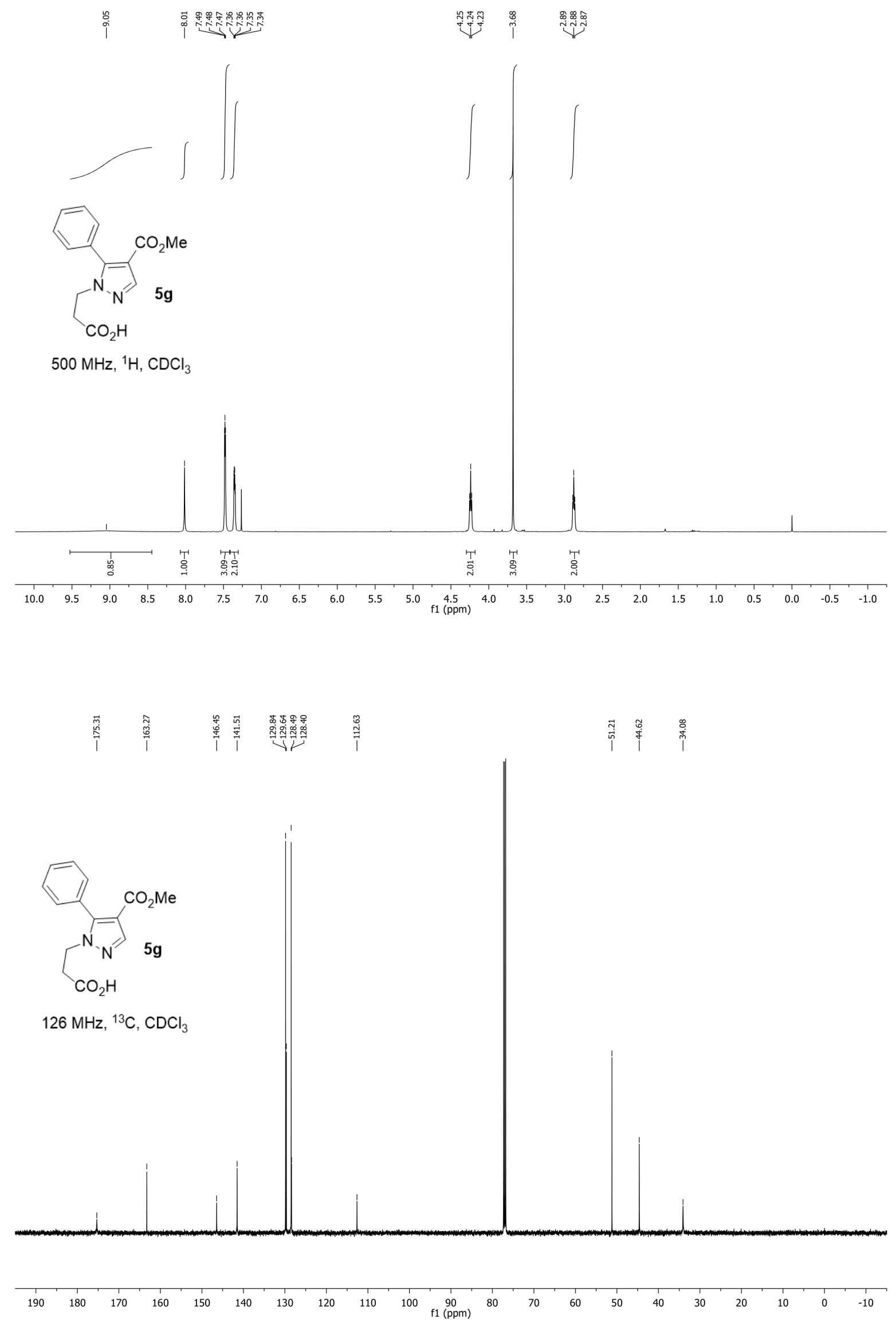

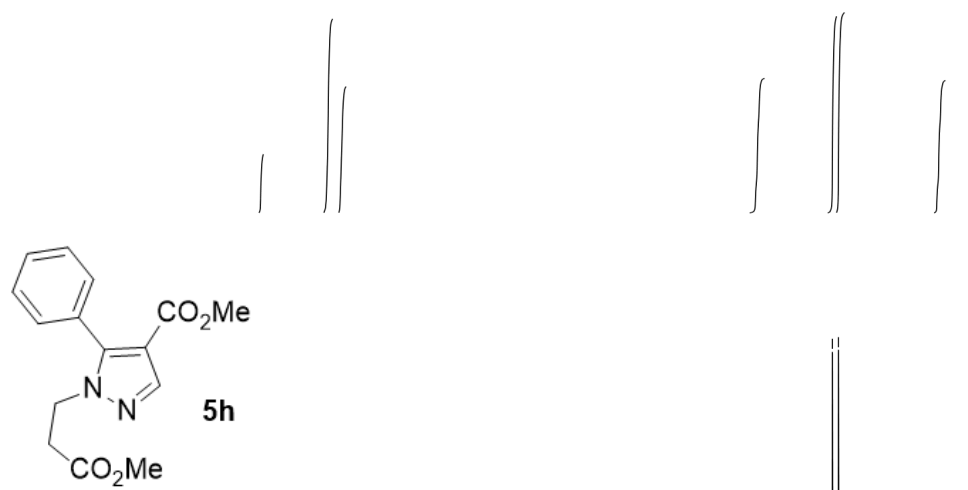

$500 \mathrm{MHz},{ }^{1} \mathrm{H}, \mathrm{CDCl}_{3}$
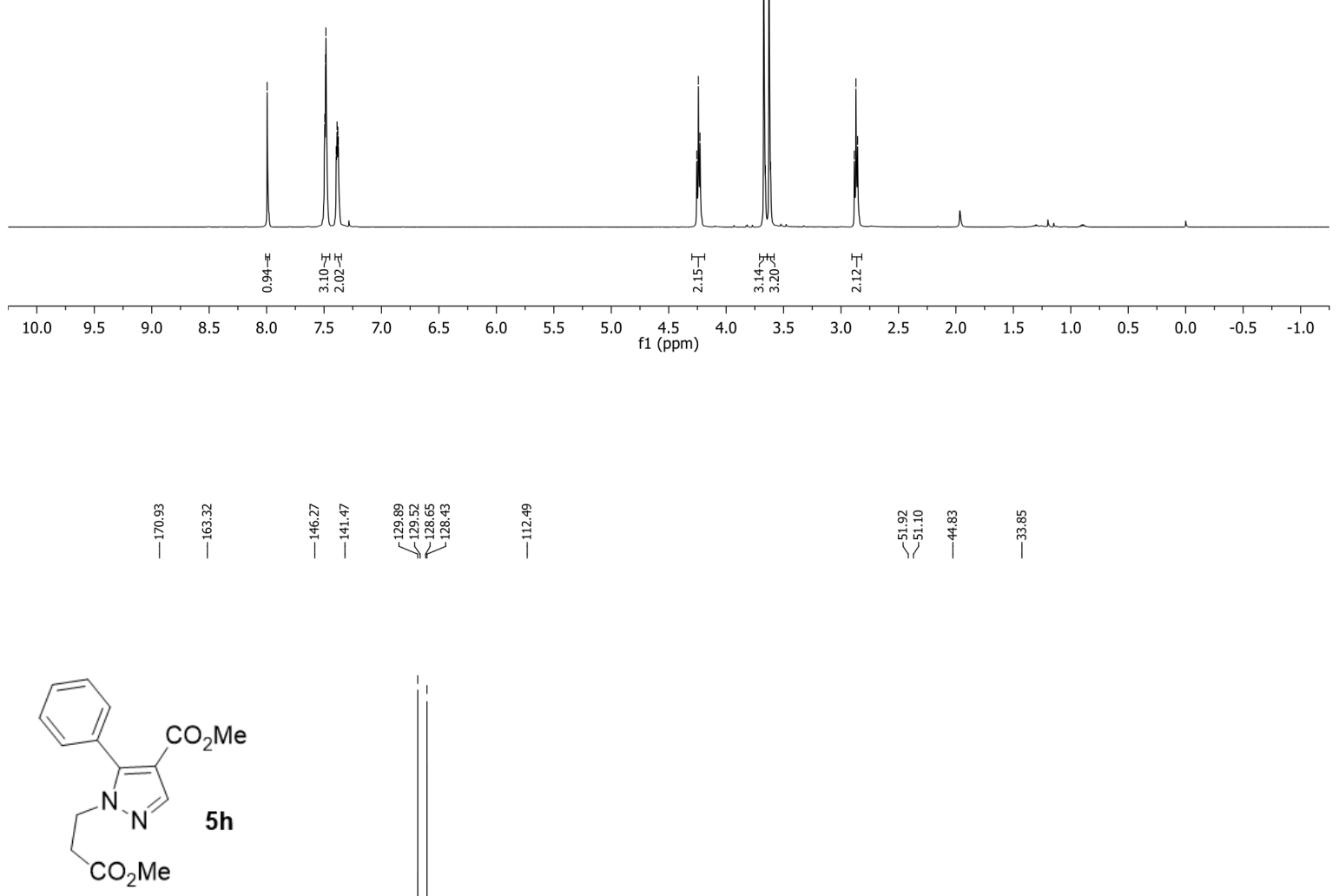

$126 \mathrm{MHz},{ }^{13} \mathrm{C}, \mathrm{CDCl}_{3}$

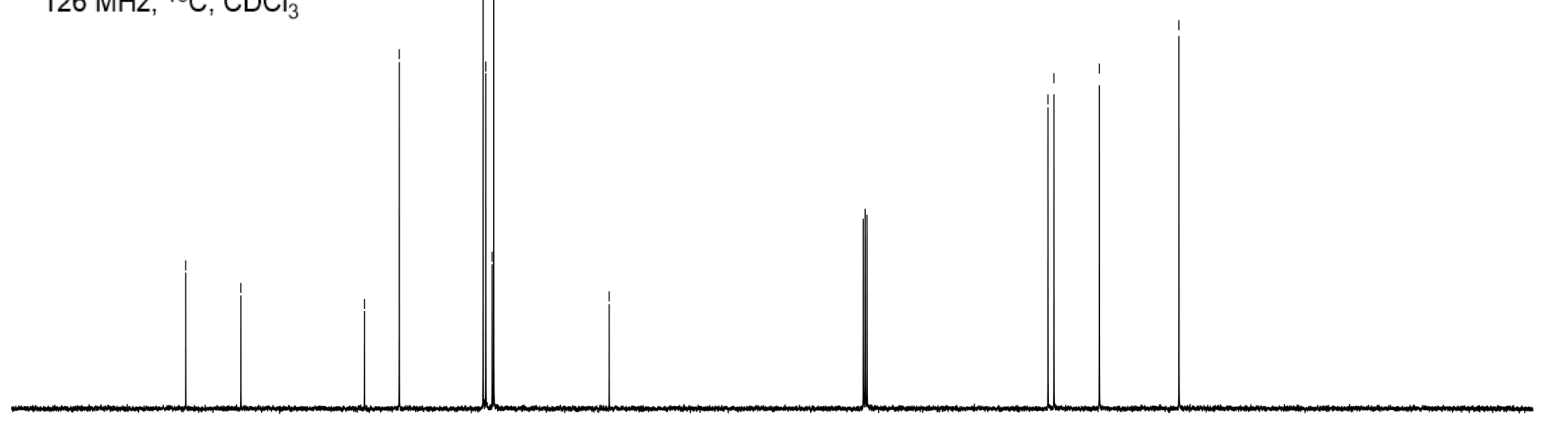

$\begin{array}{llllllllllllllllllllll}190 & 180 & 170 & 160 & 150 & 140 & 130 & 120 & 110 & 100 & 90 & 80 & 70 & 60 & 50 & 40 & 30 & 20 & 10 & 0 & -10\end{array}$ 


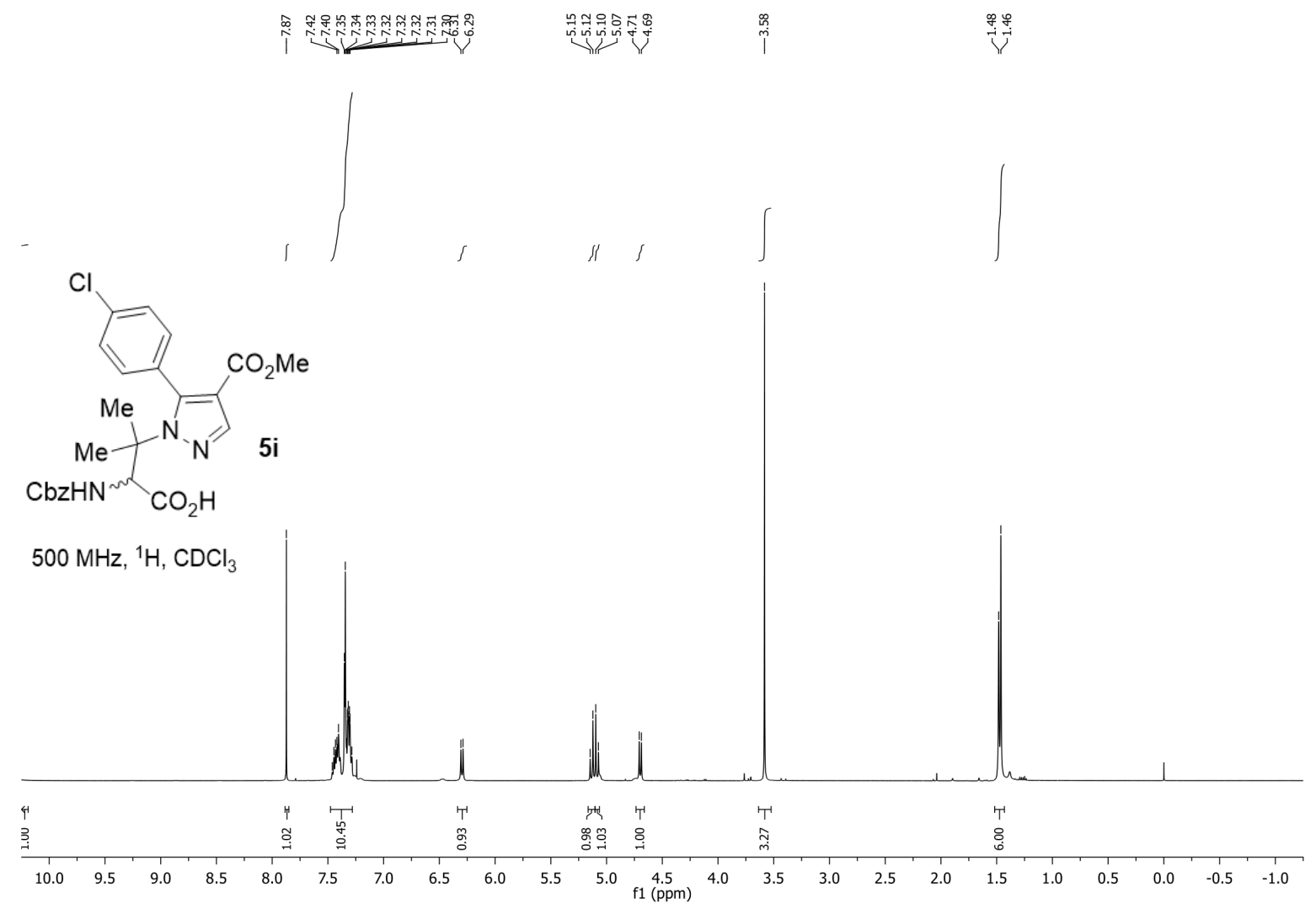

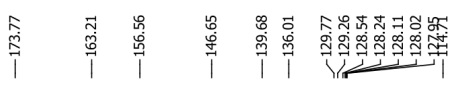

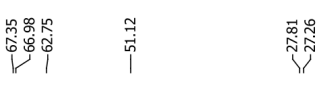

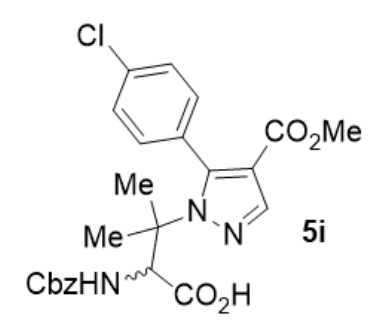

$126 \mathrm{MHz},{ }^{13} \mathrm{C}, \mathrm{CDCl}_{3}$

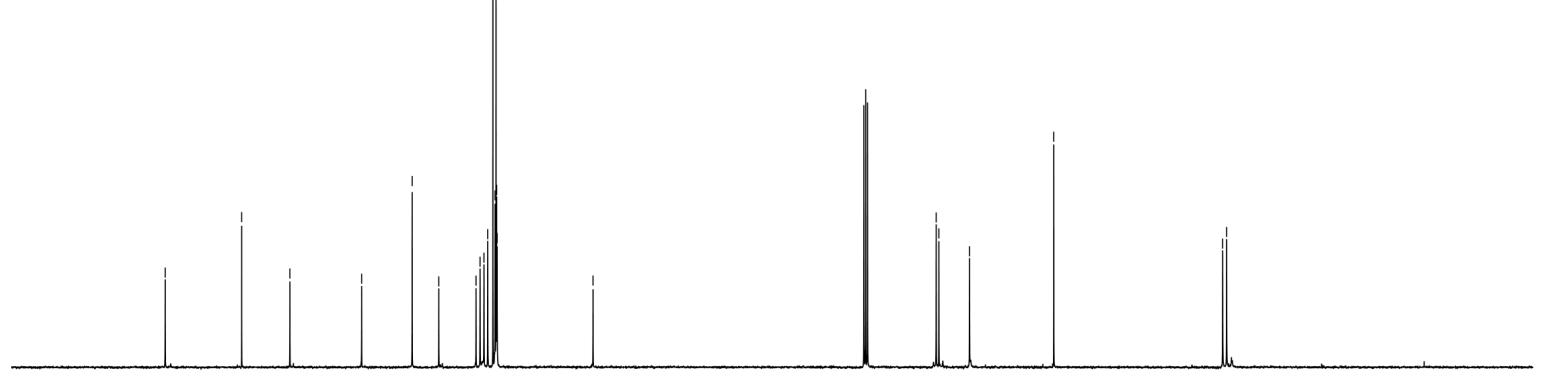

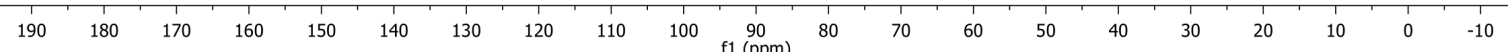



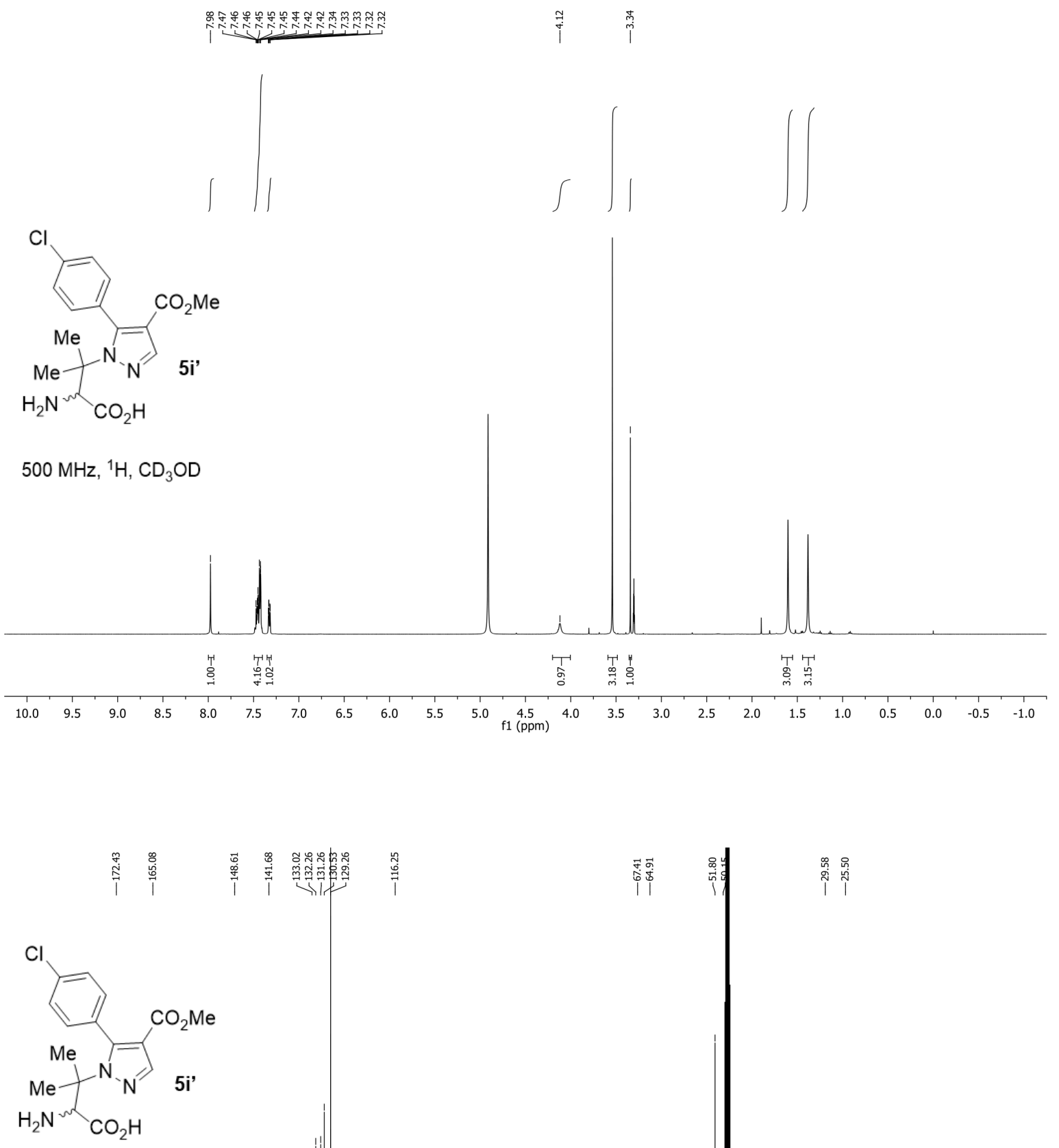

$126 \mathrm{MHz},{ }^{13} \mathrm{C}, \mathrm{CD}_{3} \mathrm{OD}$

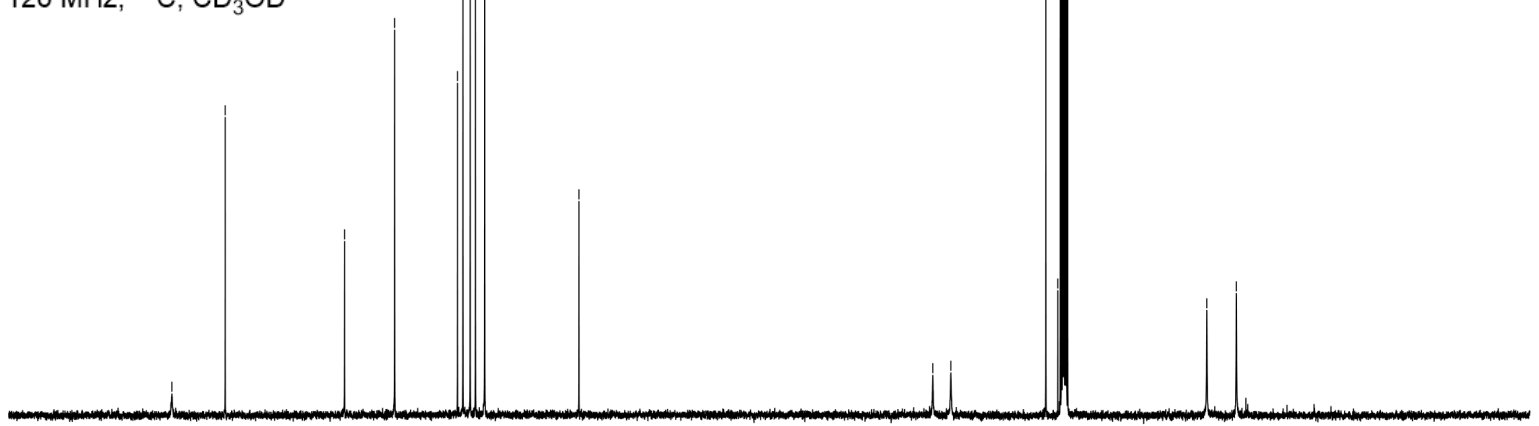

$\begin{array}{llllllllllllllllllllllll}1 & 180 & 170 & 160 & 150 & 140 & 130 & 120 & 110 & 100 & 90 & 80 & 70 & 60 & 50 & 40 & 30 & 20 & 10 & 0 & -10\end{array}$ 

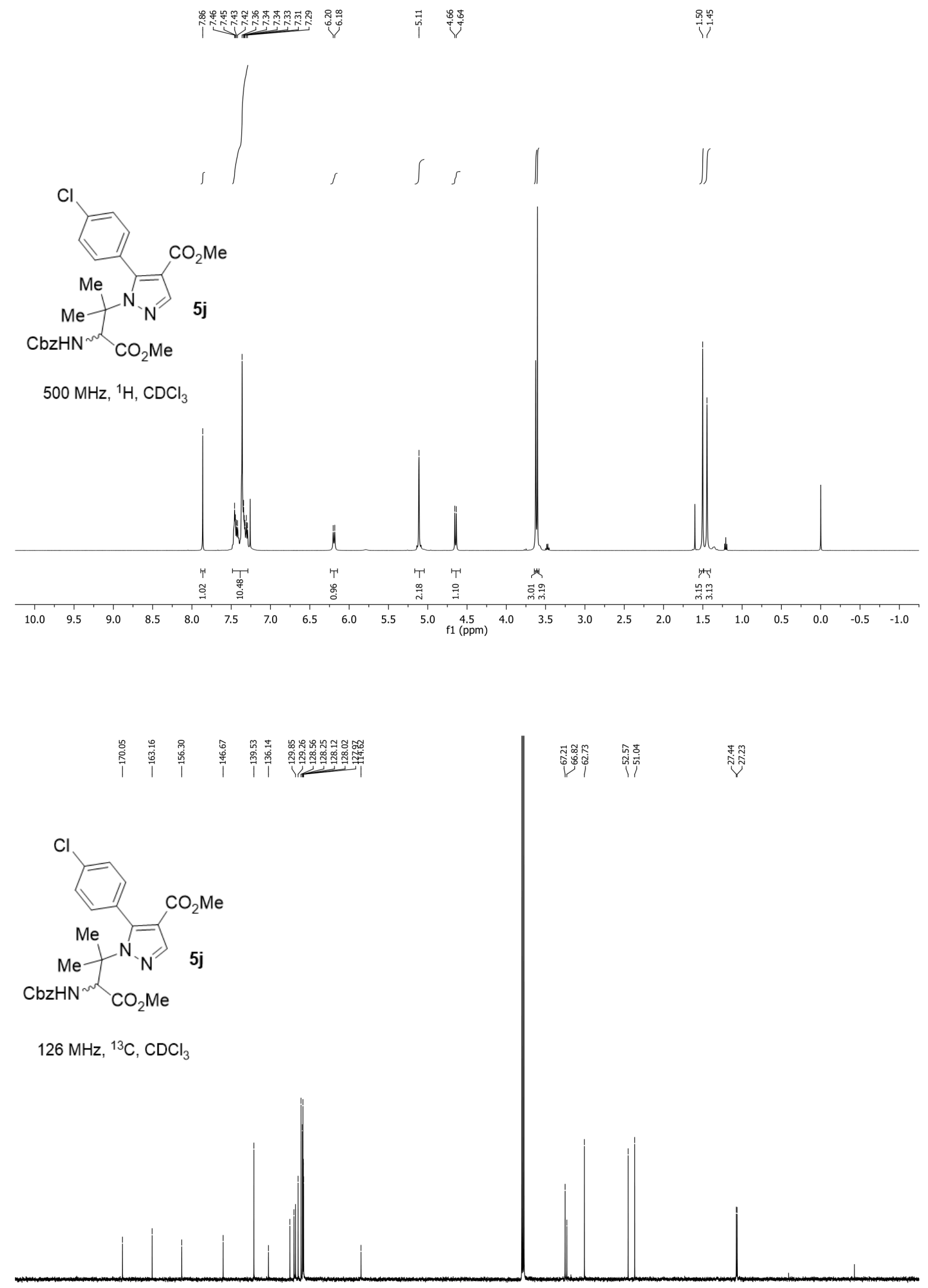

$\begin{array}{lllllllllllllllllllllllll}190 & 180 & 170 & 160 & 150 & 140 & 130 & 120 & 110 & 100 & 90 & 80 & 70 & 60 & 50 & 40 & 30 & 20 & 10 & 0 & -10\end{array}$ 


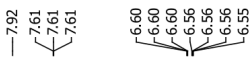

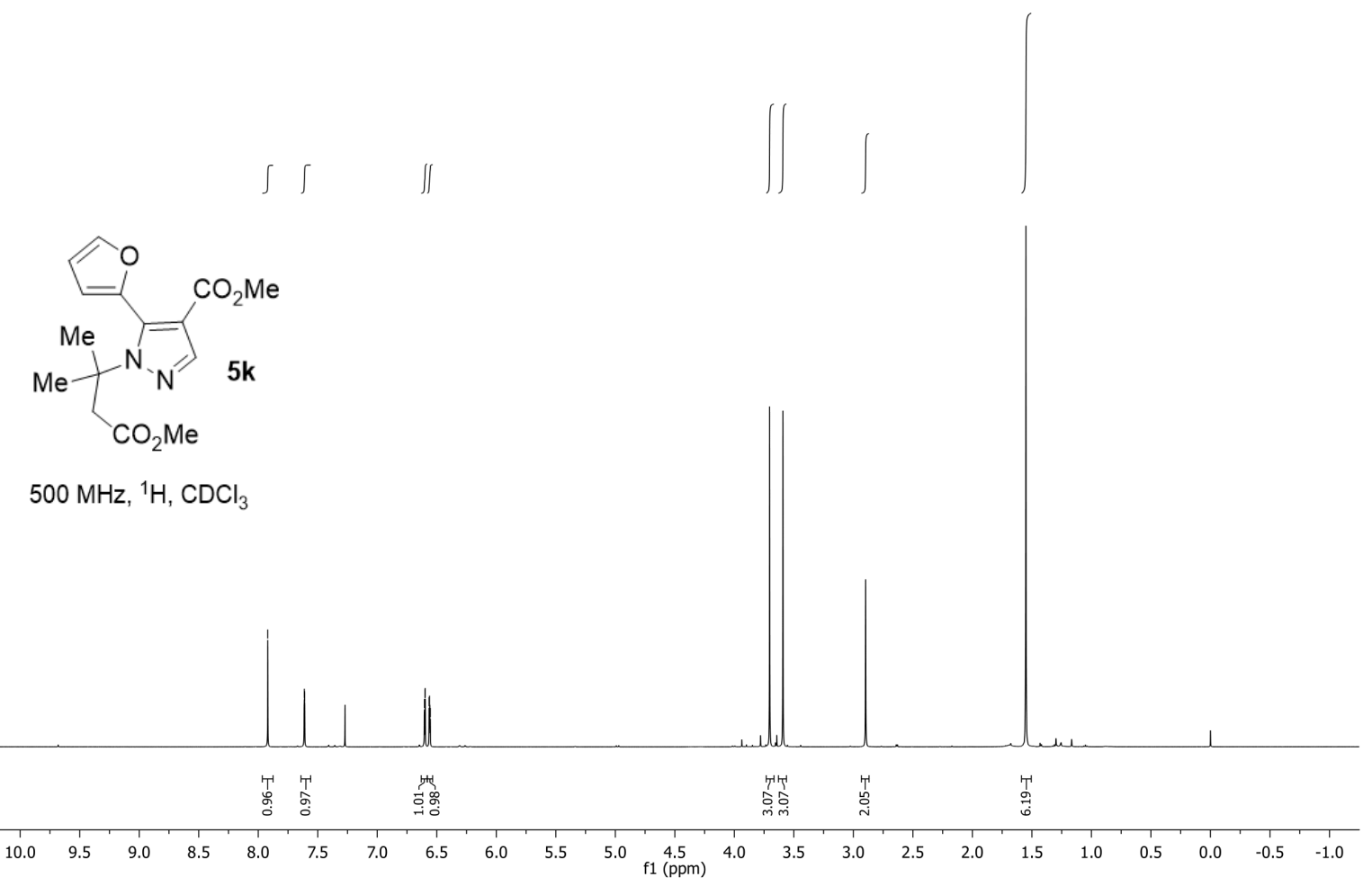

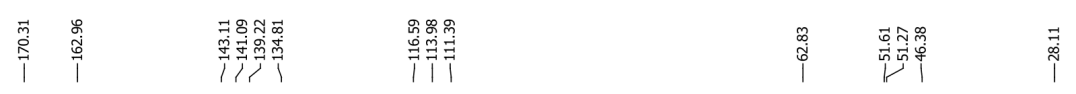

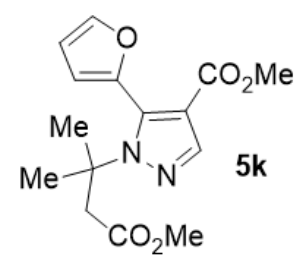

$126 \mathrm{MHz},{ }^{13} \mathrm{C}, \mathrm{CDCl}_{3}$

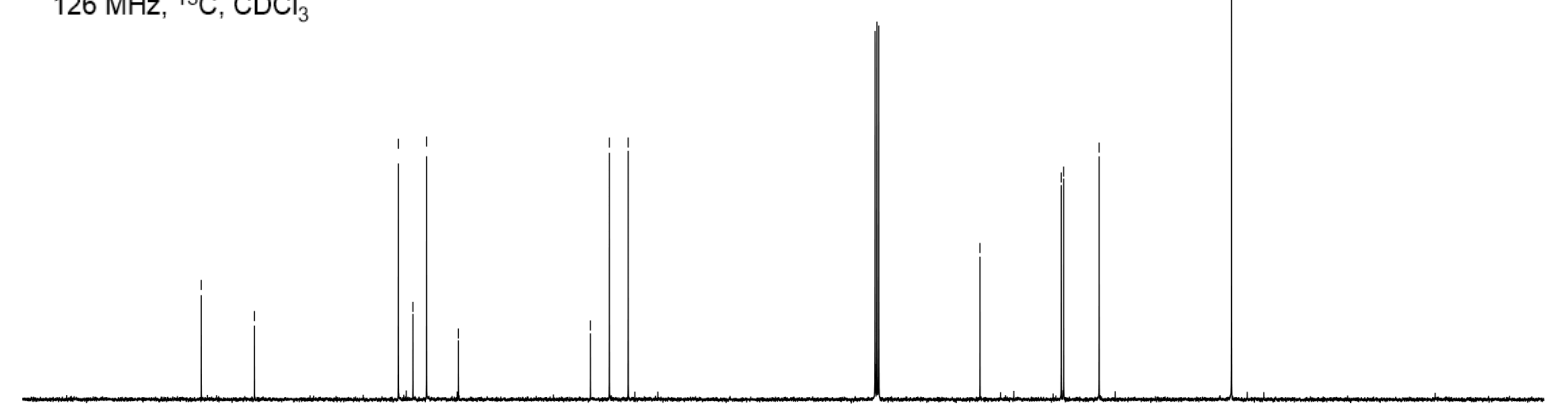

$\begin{array}{llllllllllllllllllllllll}190 & 180 & 170 & 160 & 150 & 140 & 130 & 120 & 110 & 100 & 90 & 80 & 70 & 60 & 50 & 40 & 30 & 20 & 10 & 0 & -10\end{array}$ 


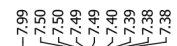

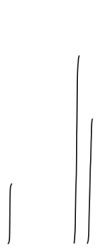

$\mathrm{CO}_{2}\left(\mathrm{CH}_{2}\right)_{4} \mathrm{Br}$

$500 \mathrm{MHz},{ }^{1} \mathrm{H}, \mathrm{CDCl}_{3}$

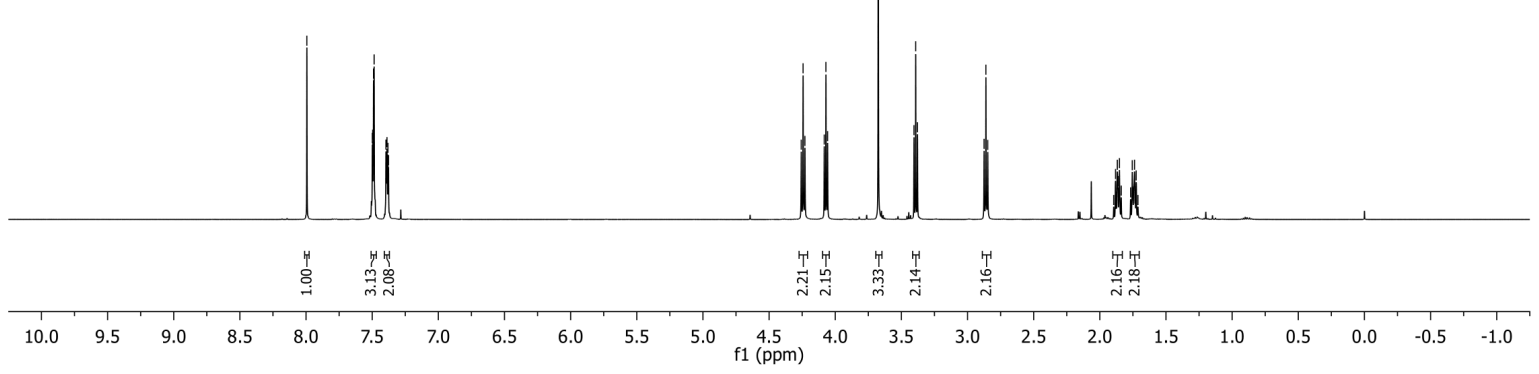

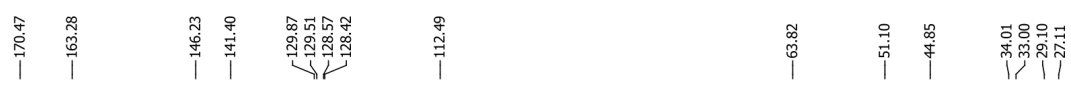

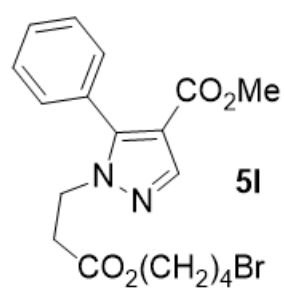

$126 \mathrm{MHz},{ }^{13} \mathrm{C}, \mathrm{CDCl}_{3}$

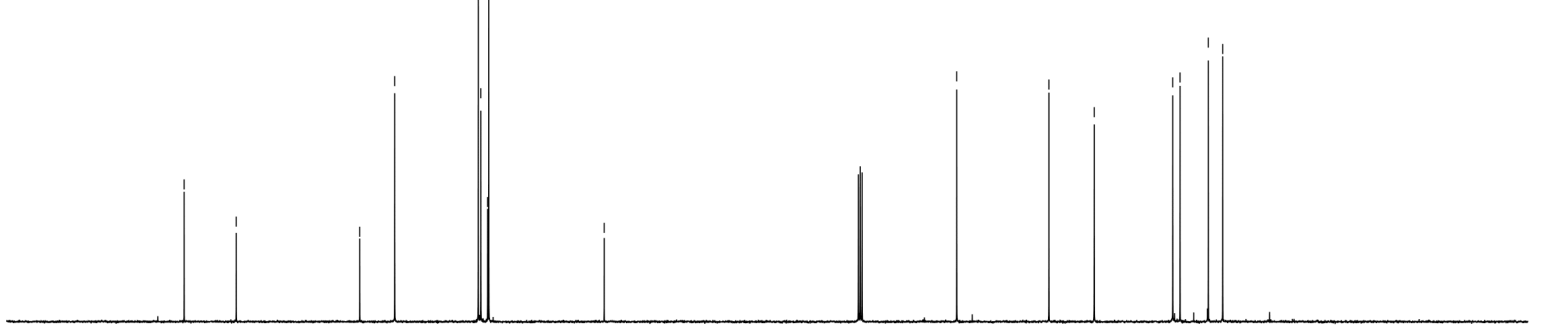

1

$190 \quad 1$

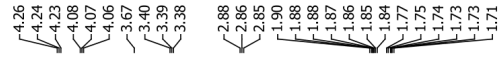

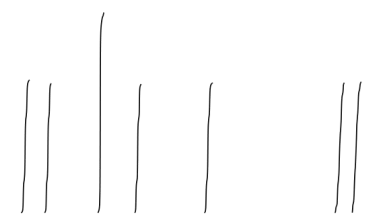



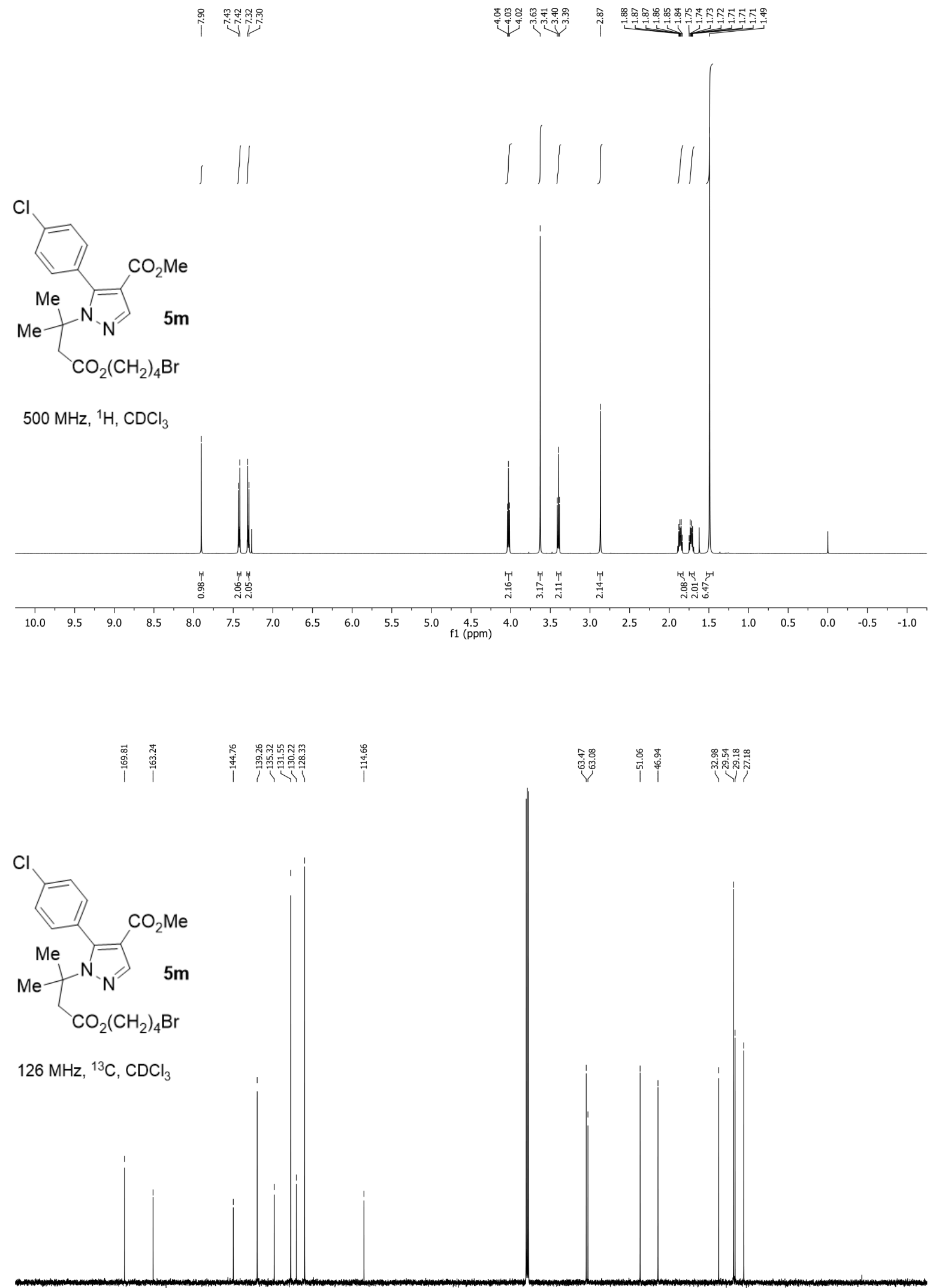

$\begin{array}{lllllllllllllllllllll}190 & 180 & 170 & 160 & 150 & 140 & 130 & 120 & 110 & 100 & 90 & 80 & 70 & 60 & 50 & 40 & 30 & 20 & 10 & 0 & -10\end{array}$ 

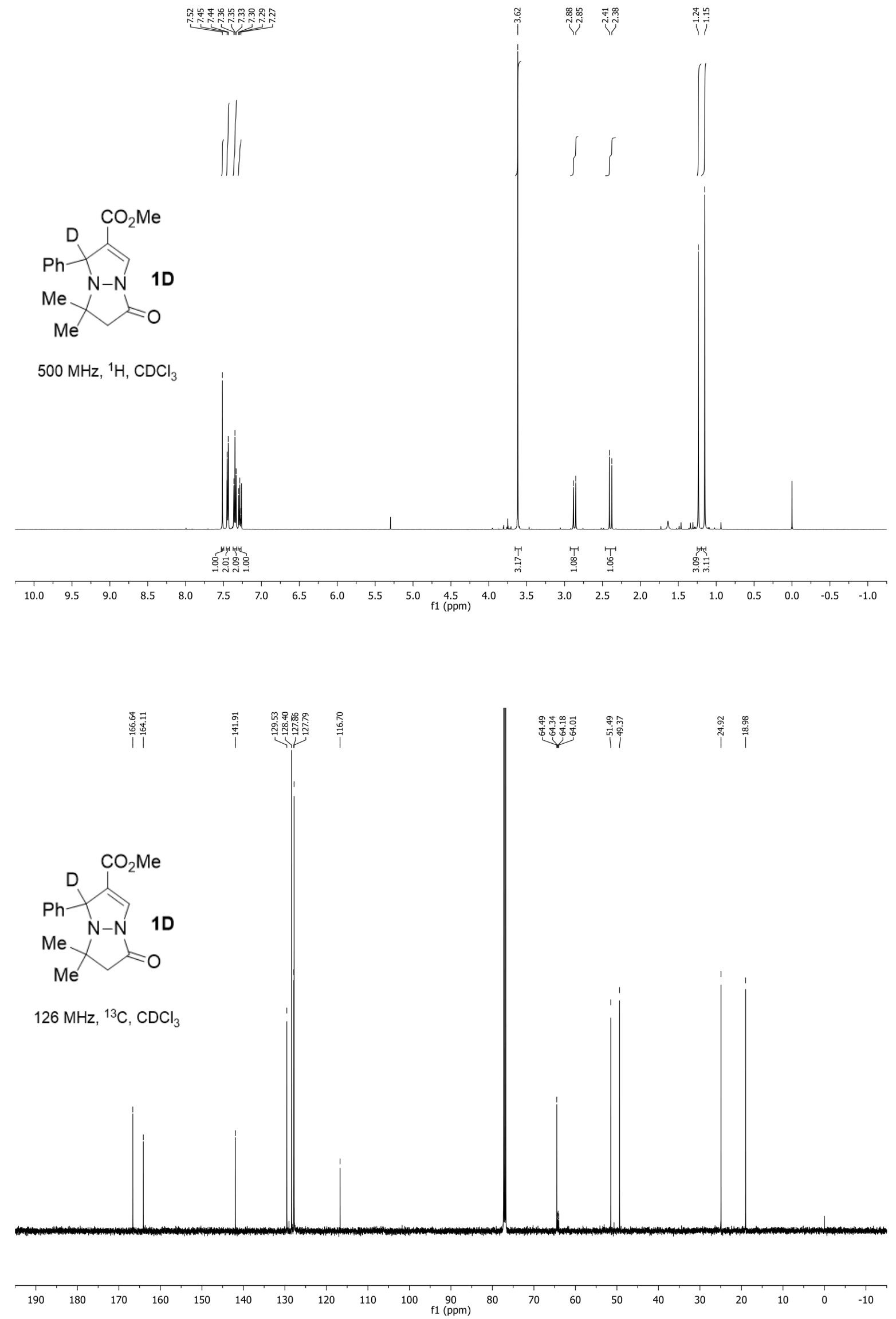

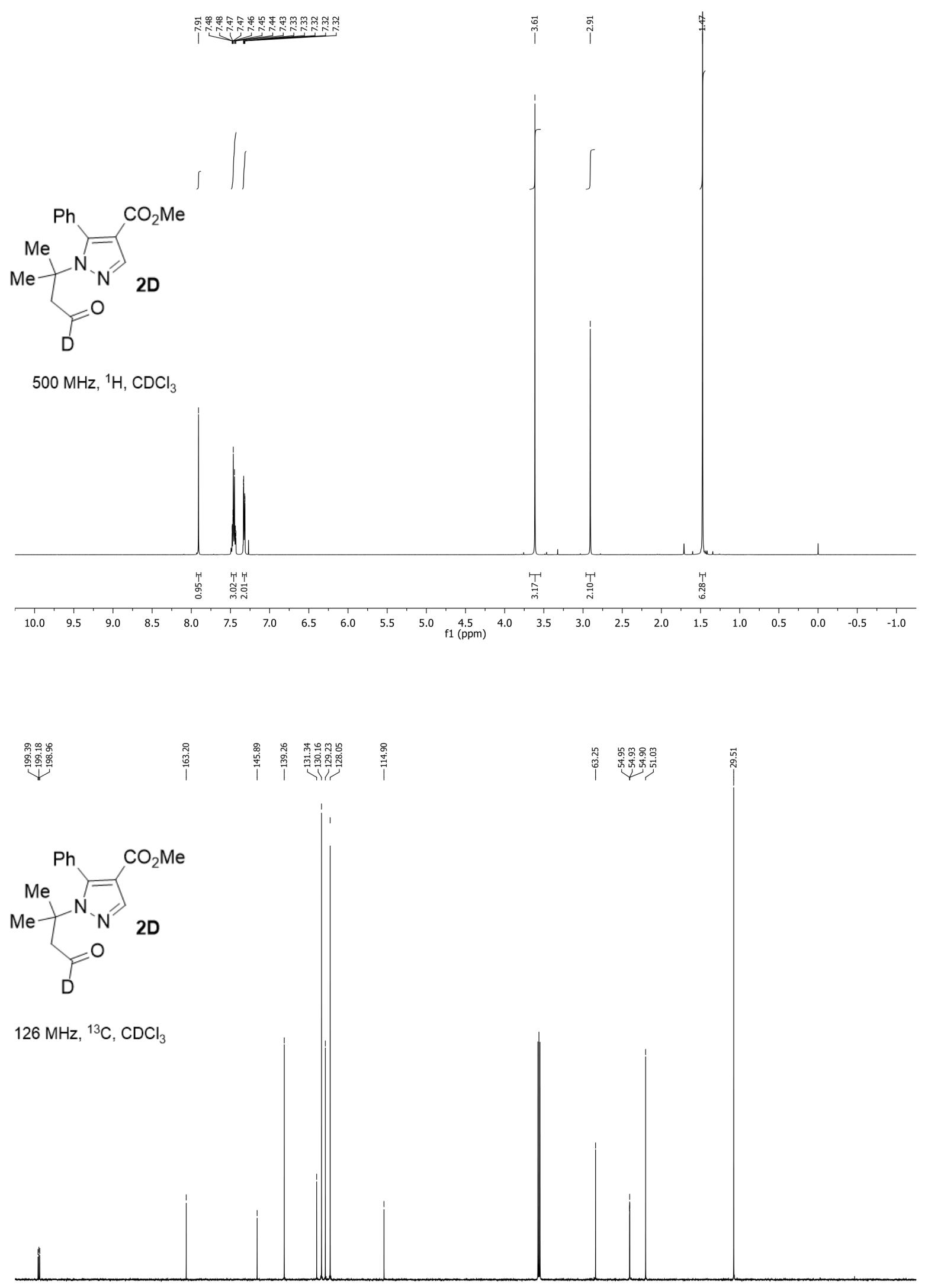

$\begin{array}{llllllllllllllllllllllllllll}200 & 190 & 180 & 170 & 160 & 150 & 140 & 130 & 120 & 110 & 100 & 90 & 80 & 70 & 60 & 50 & 40 & 30 & 20 & 10 & 0 & -10\end{array}$ 


\section{References}

1. Sibi, M. P.; Rane, D.; Stanley, L. M.; Soeta, T., Copper(II)-Catalyzed Exo and Enantioselective Cycloadditions of Azomethine Imines. Org. Lett. 2008, 10 (14), 2971-2974.

2. Schulz, M.; West, G., Photochemische Reaktionen von Pyrazolidon-(3)-betainen. II. Synthese der $\beta$-Hydrazino-isovaleriansäure. J. Prakt. Chem. 1973, 315 (4), 711-716.

3. Petek, N.; Grošelj, U.; Svete, J.; Požgan, F.; Kočar, D.; Štefane, B., Eosin Y-Catalyzed VisibleLight-Mediated Aerobic Transformation of Pyrazolidine-3-One Derivatives. Catalysts 2020, 10 (9), 981.

4. Koptelov, Y. B.; Sednev, M. V.; Kostikov, R. R., (Z)-1-alkylidene- and 1-arylmethylidene-5,5dimethyl-3-oxopyrazolidin-1-ium-2-ides and their cycloaddition to $\mathrm{N}$-arylmaleimides. Russ. J. Org. Chem. 2012, 48 (6), 804-814.

5. Taylor, E. C.; Haley, N. F.; Clemens, R. J., Synthesis and properties of 3-oxo-1,2-diazetidinium ylides. J. Am. Chem. Soc. 1981, 103 (26), 7743-7752.

6. Kawai, H.; Kusuda, A.; Nakamura, S.; Shiro, M.; Shibata, N., Catalytic Enantioselective Trifluoromethylation of Azomethine Imines with Trimethyl(trifluoromethyl)silane. Angew. Chem. Int. Ed. 2009, 48 (34), 6324-6327.

7. Turk, C.; Svete, J.; Stanovnik, B.; Golič, L.; Golič-Grdadolnik, S.; Golobič, A.; Selič, L., Regioselective 1,3-Dipolar Cycloadditions of (1Z)-1-(Arylmethylidene)-5,5-dimethyl-3oxopyrazolidin-1-ium-2-ide Azomethine Imines to Acetylenic Dipolarophiles. Helv. Chim. Acta 2001, 84 (1), 146-156.

8. Novak, A.; Štefanič, M.; Grošelj, U.; Hrast, M.; Kasunič, M.; Gobec, S.; Stanovnik, B.; Svete, J., A Simple Synthesis of Polyfunctionalized 4-Aminopyrazolidin-3-ones as 'Aza-deoxa' Analogs of D-Cycloserine. Helv. Chim. Acta 2014, 97 (2), 245-267.

9. Pušavec Kirar, E.; Grošelj, U.; Mirri, G.; Požgan, F.; Strle, G.; Štefane, B.; Jovanovski, V.; Svete, J., "Click" Chemistry: Application of Copper Metal in Cu-Catalyzed Azomethine ImineAlkyne Cycloadditions. J. Org. Chem. 2016, 81 (14), 5988-5997.

10. Takamichi, O.; Kazuaki, Y.; Kazuya, Y.; Noritaka, M., An Efficient Copper-mediated 1,3-Dipolar Cycloaddition of Pyrazolidinone-based Dipoles to Terminal Alkynes to Produce N,N-Bicyclic Pyrazolidinone Derivatives. Chem. Lett. 2010, 39 (10), 1086-1087.

11. Pušavec Kirar, E.; Grošelj, U.; Golobič, A.; Požgan, F.; Pusch, S.; Weber, C.; Andernach, L.; Štefane, B.; Opatz, T.; Svete, J., Absolute Configuration Determination of 2,3-Dihydro-1H,5Hpyrazolo[1,2-a]pyrazoles Using Chiroptical Methods at Different Wavelengths. J. Org. Chem. 2016, 81 (23), 11802-11812.

12. Mirnik, J.; Pušavec Kirar, E.; Ričko, S.; Grošelj, U.; Golobič, A.; Požgan, F.; Štefane, B.; Svete, J., Cu0-catalysed 1,3-dipolar cycloadditions of $\alpha$-amino acid derived $\mathrm{N}, \mathrm{N}$-cyclic azomethine imines to ynones. Tetrahedron 2017, 73 (24), 3329-3337.

13. Pušavec, E.; Mirnik, J.; Šenica, L.; Grošelj, U.; Stanovnik, B.; Svete, J., Cu(I)-catalyzed [3+2] Cycloadditions of tert-Butyl (S)-(3-Oxopent-4- yn-2-yl)carbamate to 1-Benzylidenepyrazole-3one-derived Azomethine Imines. Z. Naturforsch. B 2014, 69 (5), 615-626.

14. Fan, Z.; Yi, Y.; Chen, S.; Xi, C., Visible-Light-Induced Catalyst-Free Carboxylation of Acylsilanes with Carbon Dioxide. Org. Lett. 2021, 23 (6), 2303-2307.

15. Williams, A. T. R.; Winfield, S. A.; Miller, J. N., Relative fluorescence quantum yields using a computer-controlled luminescence spectrometer. Analyst 1983, 108 (1290), 1067-1071.

16. Ghosh, I.; Mukhopadhyay, A.; Koner, A. L.; Samanta, S.; Nau, W. M.; Moorthy, J. N., Excitedstate properties of fluorenones: influence of substituents, solvent and macrocyclic encapsulation. Phys. Chem. Chem. Phys. 2014, 16 (31), 16436-16445.

17. Aranzaes, J. R.; Daniel, M.-C.; Astruc, D., Metallocenes as references for the determination of redox potentials by cyclic voltammetry - Permethylated iron and cobalt sandwich complexes, inhibition by polyamine dendrimers, and the role of hydroxy-containing ferrocenes. Can. J. Chem. 2006, 84 (2), 288-299.

18. CrysAlisPRO, Agilent Technologies UK Ltd, Yarnton, Oxfordshire, England, 2011. 
19. Dolomanov, O. V.; Bourhis, L. J.; Gildea, R. J.; Howard, J. A. K.; Puschmann, H., OLEX2: a complete structure solution, refinement and analysis program. J. Appl. Crystallogr. 2009, 42 (2), 339-341.

20. Sheldrick, G., A short history of SHELX. Acta Crystallogr., Sect. A 2008, 64 (1), 112-122.

21. Sheldrick, G., SHELXT - Integrated space-group and crystal-structure determination. Acta Crystallogr., Sect. A 2015, 71 (1), 3-8.

22. Sheldrick, G., Crystal structure refinement with SHELXL. Acta Crystallogr., Sect. C 2015, 71 (1), 3-8.

23. Macrae, C. F.; Edgington, P. R.; McCabe, P.; Pidcock, E.; Shields, G. P.; Taylor, R.; Towler, M.; van de Streek, J., Mercury: visualization and analysis of crystal structures. J. Appl. Crystallogr. 2006, 39 (3), 453-457.

24. Spek, A., Single-crystal structure validation with the program PLATON. J. Appl. Crystallogr. 2003, 36 (1), 7-13. 